\title{
Influence of Pavement Reflection on Target Visibility
}

by

\author{
Ronald B. Gibbons
}

\author{
A thesis \\ presented to the University of Waterloo \\ in fulfillment of the \\ thesis requirement for the degree of \\ Doctor of Philosophy \\ in \\ Systems Design Engineering
}

Waterloo, Ontario, Canada, 1997

CRonald B. Gibbons 1997 
National Library of Canada

Acquisitions and Bibliographic Services

395 Wellington Street Ottawa ON K1A ON4 Canada
Bibliothèque nationale

du Canada

Acquisitions et

services bibliographiques

395, ne Wellington

Ottawa ON K1A ON4

Canada
The author has granted a nonexclusive licence allowing the National Library of Canada to reproduce, loan, distribute or sell copies of this thesis in microform, paper or electronic formats.
L'auteur a accordé une licence non exclusive permettant à la Bibliothèque nationale du Canada de reproduire, prêter, distribuer ou vendre des copies de cette thèse sous la forme de microfiche/film, de reproduction sur papier ou sur format électronique.

L'auteur conserve la propriété du droit d'auteur qui protège cette thèse. $\mathrm{Ni}$ la thèse ni des extraits substantiels de celle-ci ne doivent être imprimés ou autrement reproduits sans son autorisation. 
The University of Waterloo requires the signatures of all persons using or photocopying this thesis. Please sign below, and give address and date. 


\section{Influence of Pavement Reflection on Target Visibility}

\section{Abstract}

The properties of light reflecting from pavement surfaces have been studied for several years in both Europe and North America. The data has been used for the calculation of the luminance of the pavement in fixed roadway lighting design. With the proposed IESNA Standard Recommended practice RP-8 [1997], the visibility of objects on the roadway has been added as a design quality criterion rather than the pavement luminance only.

To calculate the visibility of objects on the pavement, a model of visual sensitivity is used to calculate the required contrast of an object to its background. The required contrast, called the contrast threshold, is then compared to the actual contrast of the object. The ratio of these two values is called the visibility level. The average of this visibility level, calculated for several points on the roadway, is then established as the criteria for the quality of the roadway lighting design. In order to calculate the contrast threshold and the actual contrast, both the background luminance of the target and the target luminance must be calculated. The background luminance of the target is based on a calculation which has been established for several years. The target luminance, however, is not as simple. It is calculated from both the direct light from the luminaires and the indirect light reflected from the pavement surface. Both of these calculations, the background and target luminance, require knowledge of the reflection properties of pavement surfaces. 
The pavement reflection data which currently exists is valid for a one degree observation angle only and can be used only for the background pavement luminance. It is not valid for the target luminance calculation. This research is the investigation of the surface reflection properties in order to fully describe the scatter of reflected light in all directions. Using samples of several pavement types, the reflection properties of the surfaces were measured. These measurements were then analyzed in terms of the surface characteristics.

An investigation of the surface roughness of the sample was also undertaken to investigate the possibility of relating the reflection properties to the physical properties of the pavement. No relationship was found to the reflection data but the roughness was used towards the development of an analytical model of the reflection properties.

Several methodologies have been investigated for an analytical solution of the reflection data. Some work towards a solution has been undertaken but the final model requires further investigation into the physical characteristics of the pavement.

This reflection data has been used in the development of a computer program which calculates the pavement luminance, the target luminance and the visibility level. The visibility level for several installations has been calculated. These test installations which have been studied by other researchers, have provided actual target luminance and background luminance measurements. The results of the calculations have then been compared to the data provided. It was found that the calculated contribution of the reflected 
light to the target luminance follows the same trend as the measured results. This verifies both the calculation methodology and the nature of the reflection data.

The influence of the reflected light calculation on the design weighted Visibility Level of the roadway is very small. The impact was calculated for differing road surfaces and installation types and very little influence was found. The impact of the reflected light is very significant to individual target locations. For some individual targets, the addition of the reflected light reversed the calculated target contrast and increased the visibility by as much as 4 visibility levels. The effect is very similar to that found by previous research into the verification of the visibility level calculation. Further comparisons of the calculated target luminance to actual target luminance is required to fully verify the calculations of IESNA RP-8. 


\section{Acknowledgments}

My supervisor and my friend, Dr. W. K. Adrian has been an endless and indispensable source of information and assistance. I am indebted to him for convincing me to do this research in the first place and for supporting me through the entire work. He has also been instrumental in introducing my work and myself to the industry which has opened many doors and has allowed me to become what I am.

I also wish to thank Dr. Glenn Heppler for being willing and open to step in as a cosupervisor when I needed him.

I also thank the members of my defense committee and comprehensive committees: Dr. D. Stashuk, Dr. C. MacGregor, Dr. J. Hovis, Dr. B. Wills, and Dr. G. Woo. I also thank Dr. Schmidt-Clausens for being willing to act as the external examiner for my defense.

The Philips Lighting Company has been a great support in completing this work. I need to thank my colleagues and my staff for their understanding and assistance. I must particularly recognize Dr. Dale Work. He had the confidence to hire me and the concern to allow me the freedom to complete this research. Dale has also been a kind personal support to my wife and myself for which we are grateful.

I am also indebted to Annette Dietrich and the Systems Design Graduate Office for keeping me on the straight and narrow administrative path. Similarly, the School of Optometry 
needs recognition for their willingness to accept an Engineer with open arms, giving him an office, some work and some fun.

To my working friends in Optometry, Robin Jones, Allison Zorian, Lori Campbell, and Andrew Nowinski: thanks for assistance and support.

I would also like to thank my friends and fellow graduates students, Margot, Austin, Machelle and the rest for hanging out with me.

I also must mention our Thursday night friends in Waterloo and our Tuesday night Morgantown friends: thanks for the prayer and concern.

To my in-laws, they have performed above the call of duty in all aspects of our lives. They have always been there for us whenever we needed them and have helped Heidi as I have not always been able to concentrate on the most important thing in my life.

My family has also been indispensable in helping Heidi and I. My parents have taught me well and have showed me how to excel at all things in my life. They continue to guide us along the path that we are following.

Finally, I must write the most difficult thanks of all. I do not have the words to express my thanks to my wife, Heidi. As tomorrow is our 5th wedding anniversary, I must reflect on 
the past 5 years and I wonder what she would have said if I had told her I would still be a graduate student today or that I would present her with the idea of doing a Ph.D., after promising I would never do one, on the night of one of her chemotherapy treatments. I know that in the last five years I have learned more about breast cancer and chemotherapy than I ever wanted to know, cried more than I wanted to, laughed more than I expected to, and grown more than I deserved to. She has given more to me than she has received in return, all I can do is apologize for my shortcomings, say thanks for being the better half of my whole and hope that the next five years are as great as the past five have been.

Thanks to everyone.

April 10, 1997 
This thesis, like the one before,

is dedicated to my loving wife Heidi,

because I cannot imagine accomplishing

anything without her by my side.

She is an example of courage, strength and

perseverance in times of adversity.

Although our life has been full of ups and downs,

I wouldn't change it for anything else.

February 14, 1997 


\section{The Influence of Pavement Reflection on Target Visibility Table of Contents}

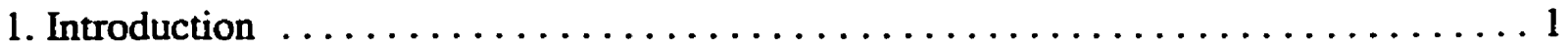

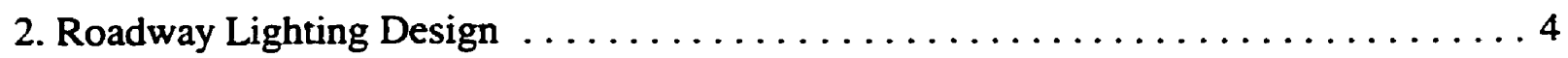

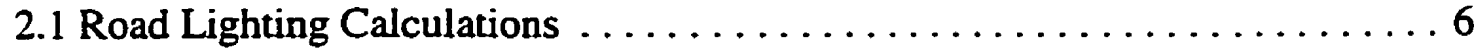

2.2 Target Luminance and the Visibility Level $\ldots \ldots \ldots \ldots \ldots \ldots \ldots \ldots$

3. Reflection Properties of Pavement Surfaces $\ldots \ldots \ldots \ldots \ldots \ldots \ldots \ldots \ldots \ldots \ldots \ldots$

3.1 Reflection Modes . . . . . . . . . . . . . . . . . . . . . 18

3.1.1 Specular Reflection $\ldots \ldots \ldots \ldots \ldots \ldots \ldots \ldots \ldots \ldots \ldots \ldots$

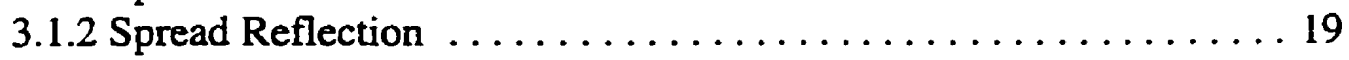

3.1.3 Diffuse Reflection .......................... 20

3.1.4 Compound Reflection ........................... 22

3.2 Pavement Reflection .............................. 24

3.2.1 CIE Road Surface Classification .................. 29

3.2.2 Proposed CIE Classification System ................. 33

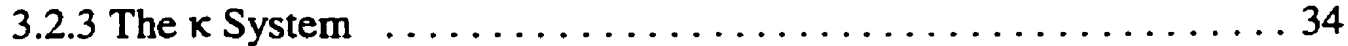

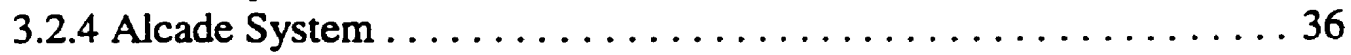

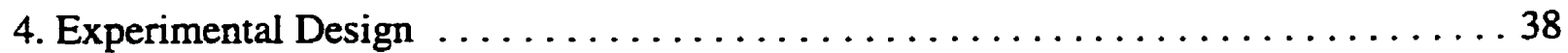

4.1 Reflection Properties Experiment $\ldots \ldots \ldots \ldots \ldots \ldots \ldots \ldots \ldots \ldots \ldots \ldots \ldots \ldots$

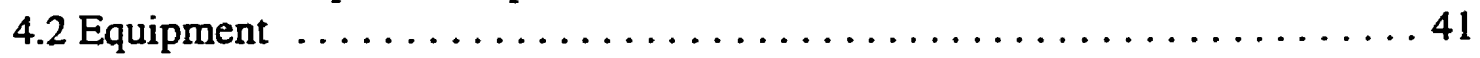

4.2.1 Samples ........................... 41

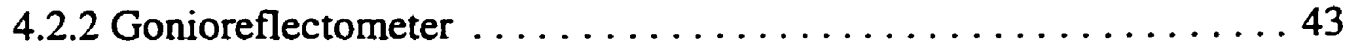

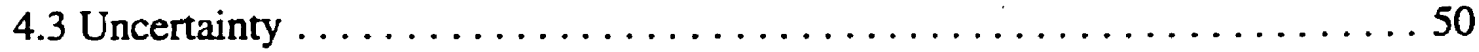

5. Reflection Properties Experiment Results . . . . . . . . . . . . . . . 62

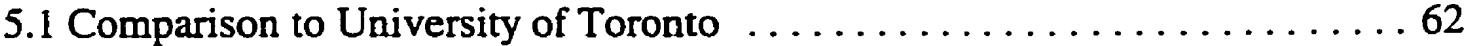

5.2 Influence of Incidence and Observation Angles $\ldots \ldots \ldots \ldots \ldots \ldots \ldots 67$

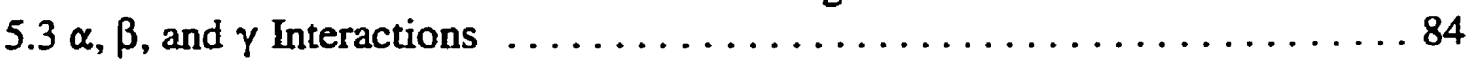

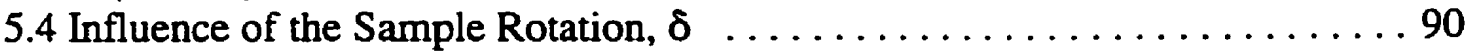

5.5 Pavement Classification System Comparisons . ................ 92

5.5 .1 CIE System . . . . . . . . . . . . . . . . . . . . 93

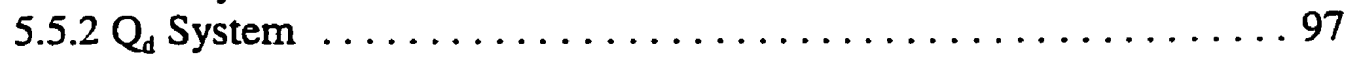

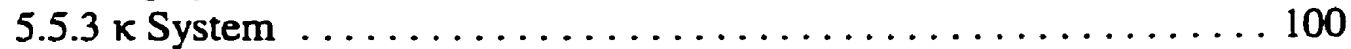

5.5 .4 Alcade System ............................ 104

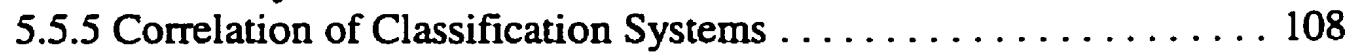

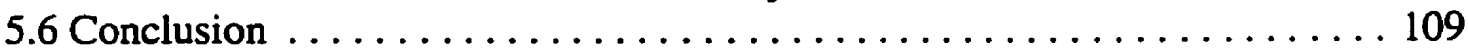

6. Surface Roughness Experiment $\ldots \ldots \ldots \ldots \ldots \ldots \ldots \ldots \ldots \ldots \ldots \ldots \ldots \ldots \ldots$ 


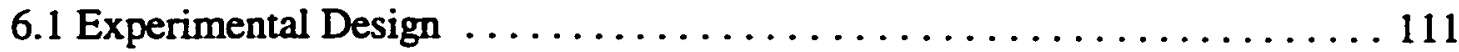

6.2 Surface Roughness Measurement $\ldots \ldots \ldots \ldots \ldots \ldots \ldots \ldots \ldots \ldots \ldots \ldots \ldots \ldots$

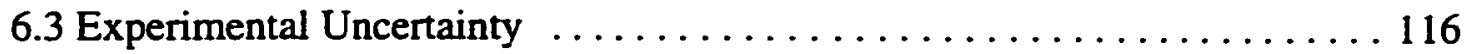

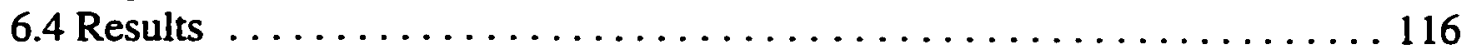

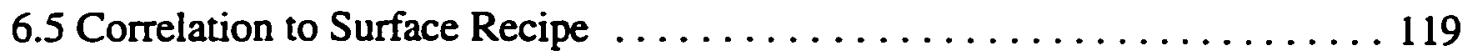

6.6 Correlation to Surface Reflection . . . . . . . . . . . . . . . . 124

6.6.1 Correlation to the CIE System ................... 124

6.6.2 Correlation to the Alcade Criteria . . . . . . . . . . . . . 126

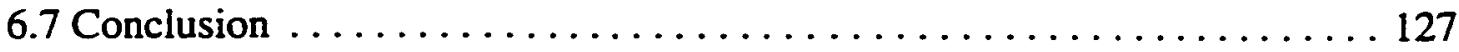

7. Analytical Modeling of Pavement Reflection .................... 129

7.1 Previous Models of Pavement Reflection . . . . . . . . . . . . . . 129

7.2 Lambertian Reflection Relationship . . . . . . . . . . . . . . . 133

7.3 Facet Model of the Reflection Profile ........................ 139

7.3.1 Model Development ........................ 139

7.3.2 Model Application ........................ 145

7.4 Physical Optics Model .............................. 157

7.4.1 Model Development ....................... 157

7.4.1.1 General Kirchoff Solution ................. 159

7.4.1.2 Normal Distribution Solution ............... 162

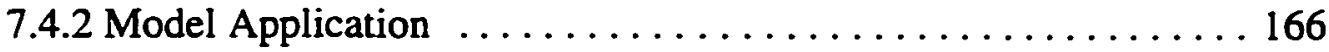

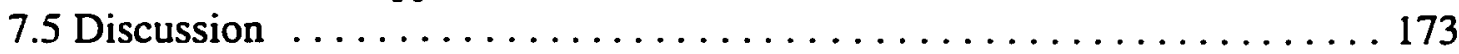

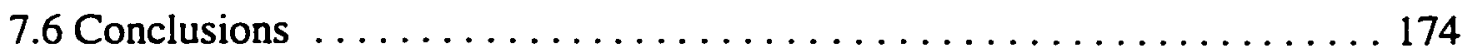

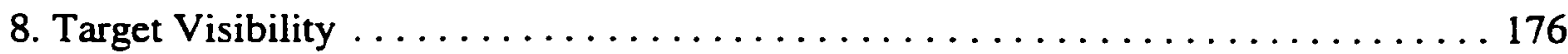

8.1 Visibility Computer Program .......................... 177

8.2 Target Luminance Calculation ....................... 182

8.2.1 Pavement Area Contributions . . . . . . . . . . . . . . . . 185

8.2.2 Pavement Area Size .......................... 189

8.2.3 Luminaire Contributions . . . . . . . . . . . . . . . . 192

8.3 Comparison to Measured Results . . . . . . . . . . . . . . . . 194

8.3.1 Philadelphia, Pennsylvania ................... 195

8.3.2 Hendersonville, North Carolina ................... 202

8.3.3 Scottsdale, Arizona . . . . . . . . . . . . . . . . . . . . . . . 206

8.3.4 Discussion ............................... 209

8.4 Impact on Visibility Level .......................... 209

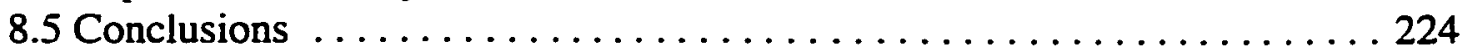

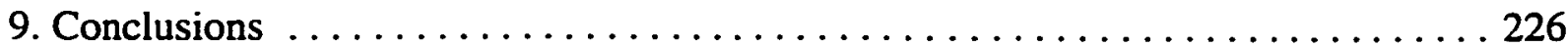

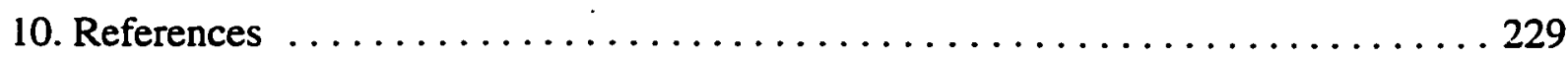

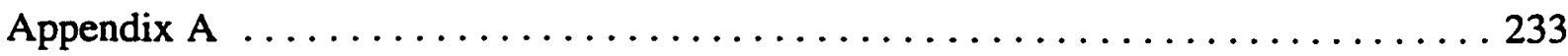




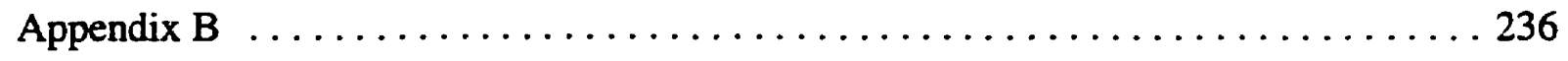

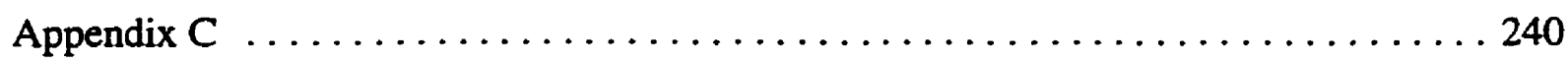

xiii 


\section{List of Tables}

Table 1 - Design Criteria for High Speed Roadways Specified in IESNA RP-8[1997] (page

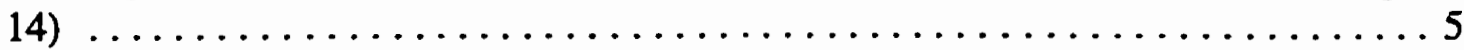

Table 2 - Road Classification Criteria Boundaries (CIE 30-2 [1982]) .......... 31

Table 3 - Pavement Surface Classifications according to IESNA RP-8 [1997] page 10 . 32

Table 4 - Errors Resulting from the Classification of Pavement Surfaces from Van

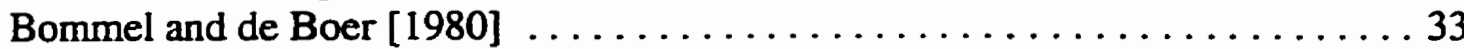

Table 5 - Classification Boundaries for the $\mathrm{k}$ System (Adrian and Enzmann [1971]) . . 35

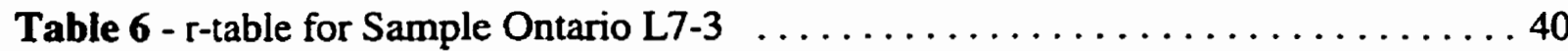

Table 7 - Selected Samples Used for the Reflection Experiment . . . . . . . . . . 42

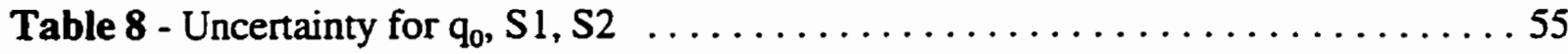

Table 9 - ANOVA for Pavement Material Type ..................... 57

Table 10 - ANOVA Table for Sample Roughness ................... 59

Table 11 - ANOVA Table for Data Collection Method ...................61 6

Table 12 - ANOVA for $\alpha, \beta$, and $\gamma$ Interactions $\ldots \ldots \ldots \ldots \ldots \ldots \ldots \ldots$

Table 13 - ANOVA for R-Class $\ldots \ldots \ldots \ldots \ldots \ldots \ldots \ldots \ldots \ldots \ldots \ldots \ldots \ldots \ldots \ldots$

Table 14 - ANOVA for R-Class, $\alpha$, and $\gamma$ Interactions $\ldots \ldots \ldots \ldots \ldots \ldots \ldots$

Table 15 - ANOVA for R-Class, $\gamma$ and $\beta$ Interactions $\ldots \ldots \ldots \ldots \ldots \ldots \ldots$

Table 16 - ANOVA for R-Class, $\alpha$ and $\beta$ Interactions $\ldots \ldots \ldots \ldots \ldots \ldots \ldots$

Table 17 - ANOVA Table for $\delta$ Influence $\ldots \ldots \ldots \ldots \ldots \ldots \ldots \ldots \ldots . \ldots . \ldots . \ldots 9$

Table 18 - Correlation of CIE and Alcade Classification Parameters ............ 108

Table 19 - Results of the Surface Roughness Experiment ................ 119

Table 20 - Material Composition of Samples ..................... 120

Table 21 - Fields in the Material Composition Table .................. 121

Table 22 - Correlation of Surface Roughness Characteristics to the Material Recipe . 121

Table 23 - Correlation of Surface Roughness Characteristics to CIE Reflection Parameters

Table 24 - Correlation of Surface Roughness Characteristics to Alcade Reflection

Parameters .................................. 126

Table 25 - Correlation of Diffuse Model Parameters to Pavement Characteristics ... 151

Table 26 - Linear Regression Results for the Physical Optics Model ........... 172

Table 27 - Correlation Coefficients of Linear Regression Parameters to Surface

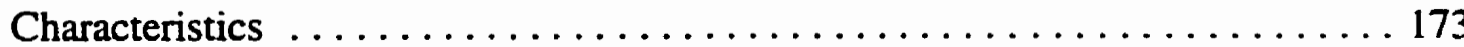

Table 28 - Target Luminance Results for Sample Calculation ............. 185

Table 29 - Comparison of Indirect Contribution of Large to Small Reflection Areas . 192

Table 30 - Comparison of Measured Iluminance to Scaled Calculated Iluminance for

Philadelphia Experiment .......................... 197

Table 31 - Comparison of Measured Pavement Luminance to Calculated Pavement

Luminance for Philadelphia Experiment ..................... 199

Table 32 - Comparison of Measured Target Luminance to Calculated Target Luminance

Table 33 - Comparison of Calculated Results to Measured for Hendersonville Experiment 
Table 34 - Comparison of Measured to Calculated Results for Scottsdale Experiment

Table 35 - Visibility Results for Single-Sided Arrangement without Indirect Target 208

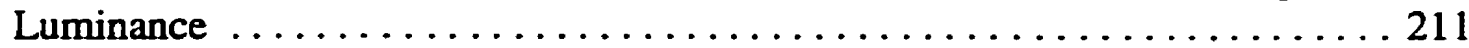

Table 36 - Visibility Results for Single-Sided Arrangement with Indirect Target

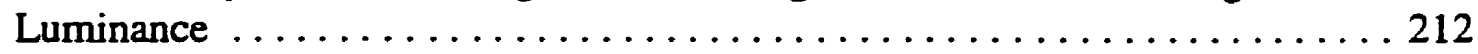

Table 37 - Visibility Results for Opposite Arrangement with Indirect Target Luminance

........................................ 215

Table 38 - Visibility Results for Opposite Arrangement without Indirect Target Luminance

...................................... 216

Table 39 - Visibility Results for Staggered Arrangement with Indirect Target Luminance

Table 40 - Visibility Results for Staggered Arrangement without Indirect Target

Luminance .............................. 220

Table 41 - Weighted Visibility Level Results for Different Luminaire Arrangements and

Calculation Methods 223 


\section{List of Figures}

Figure 1 - Geometry for Calculating Pavement Luminance $\ldots \ldots \ldots \ldots \ldots \ldots \ldots$

Figure 2 - Sources of Target Luminance . . . . . . . . . . . . . . . . . 14

Figure 3 - Geometry for the Indirect Target Iluminance Calculation $\ldots \ldots \ldots \ldots \ldots$

Figure 4 - Specular Reflection Profile . . . . . . . . . . . . . . . . . 19

Figure 5 - Spread Reflection Profile ............................ 20

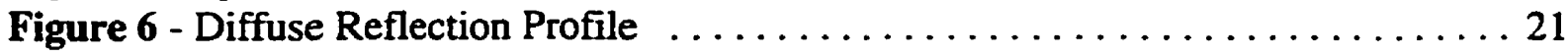

Figure 7 - Relationship of Luminance to Intensity $\ldots \ldots \ldots \ldots \ldots \ldots \ldots \ldots \ldots \ldots \ldots \ldots \ldots$

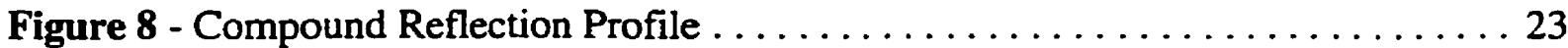

Figure 9 - Geometry of the BRDF . . . . . . . . . . . . . . . . . 24

Figure 10 - Contribution of Pavement Facets to the Reflection Profile .......... 26

Figure 11 - Local Reflection and Incidence Angles from a Single Stone in the Pavement

Surface from CIE [1995] . . . . . . . . . . . . . . . . . . . 26

Figure 12 - Macro Roughness (Top Diagram) and Micro Roughness (Bottom Diagram)

Figure 13 - The Reflection Profile and the Variables Used for the $\kappa$ Calculation . . . . 35

Figure 14 - Angular Limits of Reflection onto a Target Face . . . . . . . . . . . . 39

Figure 15 - Limits of Measurement for a Pavement Sample ............... 39

Figure 16 - Schematic of the Gonioreflectometer . . . . . . . . . . . . . . . 44

Figure 17 - Lamp Stabilization Curve . . . . . . . . . . . . . . . . . . 47

Figure 18 - Calibration of Gonioreflectometer with Magnesium Sulphate Reflectance

Standard ................................... 49

Figure 19 - Average Normalized Uncertainty in $\alpha \ldots \ldots \ldots \ldots \ldots \ldots \ldots \ldots \ldots \ldots$

Figure 20 - Average Normalized Uncertainty in $\beta \ldots \ldots \ldots \ldots \ldots \ldots \ldots \ldots \ldots$

Figure 21 - Average Normalized Uncertainty in $\gamma \ldots \ldots \ldots \ldots \ldots \ldots \ldots . \ldots . \ldots . \ldots . \ldots$

Figure 22 - Percent Uncertainty Comparison for Different Materials Types ....... 56

Figure 23 - Percent Uncertainty Comparison for Different Roughness Samples ...... 58

Figure 24 - Percent Uncertainty Comparison for Data Collection Methodologies . . . . 60

Figure 25 - Comparison of S1, S2, $q_{0}$ for University of Toronto Results and Current

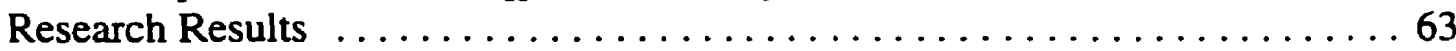

Figure 26 - Comparison of the Influence of $\gamma$ from the University of Toronto and this

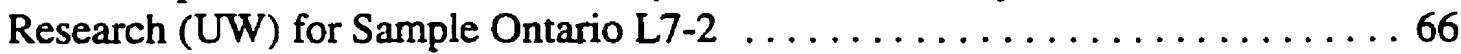

Figure 27 - Comparison of the Change in $\beta$ Results from the University of Toronto Data to

this Research (UW) for Sample Saskatchewan $10 \ldots \ldots \ldots \ldots \ldots \ldots \ldots 67$

Figure 28 - Influence of $\alpha$ on the Reflection Profile for RI Class Samples .........6 69

Figure 29 - Influence of $\alpha$ on the Reflection Profile for R2 Class Samples ........ 70

Figure 30 - Influence of $\alpha$ on the Reflection Profile for R3 Class Samples ........ 71

Figure 31 - Influence of $\alpha$ on the Reflection Profile for R4 Class Samples ........ 72

Figure 32 - Influence of $\beta$ on the Reflection Profile for RI Class Samples ........ 73

Figure 33 - Influence of $\beta$ on the Reflection Profile for R2 Class Samples ........ 74

Figure 34 - Influence of $\beta$ on the Reflection Profile for R3 Class Samples ........ 75 
Figure 35 - Influence of $\beta$ on the Reflection Profile of R4 Class Pavements ... . . 76

Figure 36 - Influence of $\gamma$ on the Reflection Profile for R1 Class Samples . . . . . . 77

Figure 37 - Influence of $\gamma$ on the Reflection Profile for R2 Class Samples . . . . . . 78

Figure 38 - Influence of $\gamma$ on the Reflection Profile for R3 Class Samples . . . . . . 79

Figure 39 - Influence of $\gamma$ on the Reflection Profile of R4 Class Samples . . . . . . 80

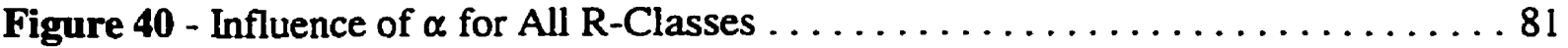

Figure 41 - Influence of $\beta$ for All R-Classes $\ldots \ldots \ldots \ldots \ldots \ldots \ldots \ldots \ldots \ldots \ldots \ldots$

Figure 42 - Influence of $\gamma$ for All R-Classes $\ldots \ldots \ldots \ldots \ldots \ldots \ldots \ldots \ldots \ldots \ldots$

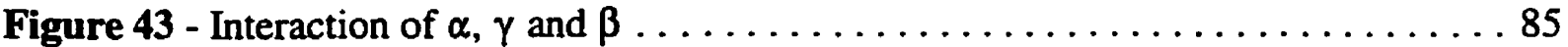

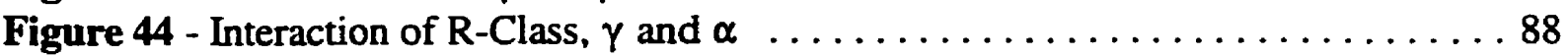

Figure 45 - Interaction of R-Class, $\alpha$, and $\beta \ldots \ldots \ldots \ldots \ldots \ldots \ldots \ldots \ldots \ldots \ldots$

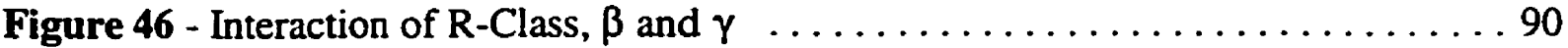

Figure 47 - Ratio of Measured Luminance to Average Measurement for Varying $\delta$. . 91

Figure 48 - Change in $q_{0}$ with Observation Angle, $\alpha$, for All R-Classes . . . . . . . 94

Figure 49 - Change in $\mathrm{SI}$ with Observation Angle for all R-Classes . . . . . . . . . 95

Figure 50 - Change in $\mathrm{S} 2$ with Observation Angle, $\alpha \ldots \ldots \ldots \ldots \ldots \ldots \ldots$

Figure 51 - Influence of Observation Angle on $Q_{d} \ldots \ldots \ldots \ldots \ldots \ldots$

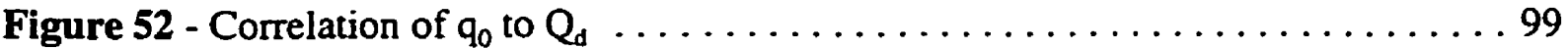

Figure 53 - Influence of Observation Angle, $\alpha$, on $\mathbf{k} \ldots \ldots \ldots \ldots \ldots \ldots \ldots \ldots$

Figure 54 - Influence of Observation Angle, $\alpha$ on $k_{p} \ldots \ldots \ldots \ldots \ldots \ldots \ldots \ldots$

Figure 55 - Influence of Observation Angle, $\alpha$, on $A_{D} \ldots \ldots \ldots \ldots \ldots \ldots$

Figure 56 - Influence of Observation Angle, $\alpha$, on the $A_{11}$ Parameter $\ldots \ldots \ldots 106$

Figure 57 - Influence of Observation Angle, $\alpha$, on parameter $A_{12} \ldots \ldots \ldots \ldots$

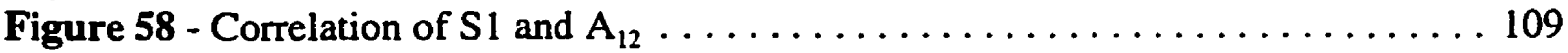

Figure 59 - Surface Height and Autocorrelation Distance $\ldots \ldots \ldots \ldots \ldots \ldots \ldots 112$

Figure 60 - Plan and Profile Schematic of Surface Roughness Pantograph . . . . . 114

Figure 61 - Calibration of the Pantograph $\ldots \ldots \ldots \ldots \ldots \ldots \ldots \ldots \ldots \ldots \ldots \ldots \ldots$

Figure 62 - Roughness Profile for Saskatchewan \# $10 \ldots \ldots \ldots \ldots \ldots \ldots \ldots$

Figure 63 - Comparison of Roughness Profile for Manitoba 200 and New Brunswick 5

Figure 64 - Calculation of the Facet Angle $\ldots \ldots \ldots \ldots \ldots \ldots \ldots \ldots \ldots \ldots \ldots$

Figure 65 - Correlation of Percent Coarse Aggregate to Average Surface Roughness . 123

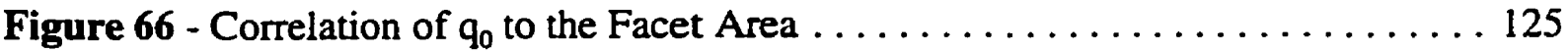

Figure 67 - Correlation of $A_{D}$ to Average Surface Roughness . . . . . . . . . 127

Figure 68 - $\alpha$ versus Reflected Luminance with the Lambertian Reflection Relationship

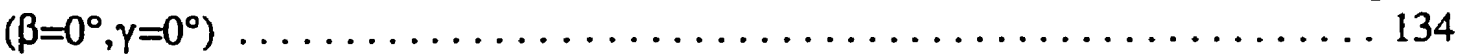

Figure 69 - Pavement Luminance versus $\gamma$ with Lambertian Reflection Relationship with

the Reduction in Incident Intensity $\left(\alpha=60^{\circ}, \beta=0^{\circ}\right) \ldots \ldots \ldots \ldots \ldots \ldots$

Figure 70 - Pavement Luminance versus $\beta$ with Lambertian Reflection Profile $\left(\alpha=60^{\circ}\right.$,

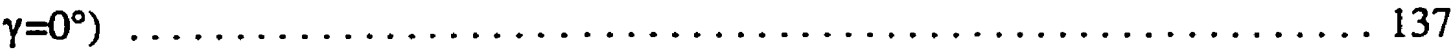

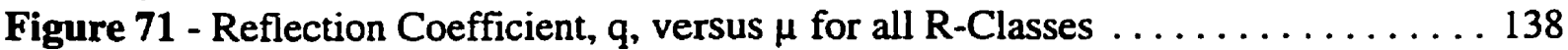

Figure 72 - Angles for Facet Model Derivation from Vermeulen [1975] . . . . . . 140

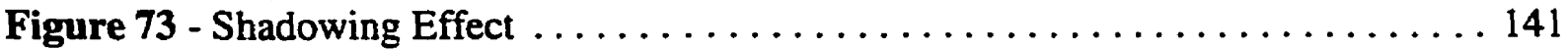


Figure 74 - Angle Definition for the Diffuse Reflection Model ............. 143

Figure $75-D(\varphi)$ for All Samples ................................ 146

Figure 76 - Diffuse Shadowing Function of R1 Road Class .............. 147

Figure 77 - Diffuse Shadowing Function for R2 Road Class . . . . . . . . . . 148

Figure 78 - Diffuse Shadowing Function for R3 Road Class ............... 149

Figure 79 - Diffuse Shadowing Function for R4 Road Class .............. 150

Figure 80 - Relationship of the Specular Component of Reflection to $\alpha$ for the 4 R-Classes

Figure 81 - Relationship of the Specular Component of Reflection to $\beta$ for the 4 R-Classes

Figure 82 - Relationship of the Specular Component of Reflection to $\gamma$ for the 4 R-Classes

Figure 83 - Relationship of the Specular Component of Reflection to i . . . . . . . . 155

Figure 84 - Relationship of the Specular Component of Reflection to $\varepsilon \ldots \ldots \ldots 156$

Figure 85 - Rayleigh Criterion Geometry . . . . . . . . . . . . . . . . 158

Figure 86 - Calculated Results for Physical Optics Model in terms of $\alpha$ for Sample Ontario

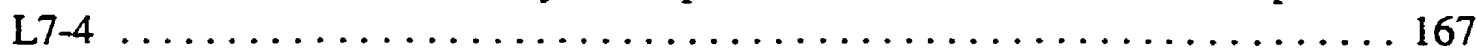

Figure 87 - Calculated Results for Physical Optics Model in terms of $\beta$ for Sample Ontario

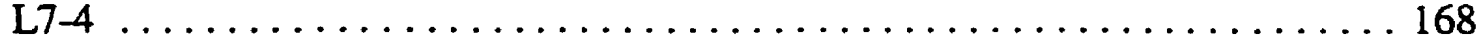

Figure 88 - Calculated Results for Physical Optics Model in terms of $\gamma$ for Sample Ontario

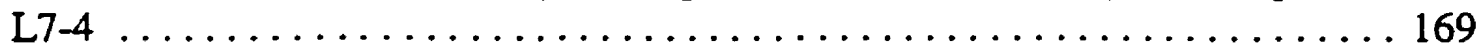

Figure 89 - Comparison of Reflection Results and Calculated Physical Optics Model for

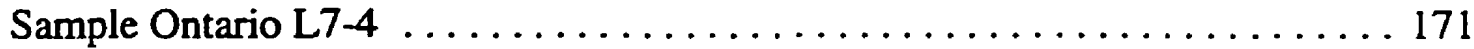

Figure 90 - Algorithm of the Pavement Luminance Calculation Program ........ 179

Figure 91 - Sample Installation Used for Establishing the Contribution of Reflected Light

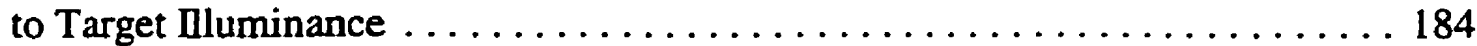

Figure 92 - Sample Indirect Calculation Grid .................... 186

Figure 93 - Total Indirect Contribution to Target Mluminance in lux .......... 187

Figure 94 - Indirect Contribution of Luminaire 4 to Target 1 Illuminance ........ 188

Figure 95 - Indirect Contribution of Luminaire 10 to Target 1 Iluminance ..... . . 189

Figure 96 - Indirect Target Mluminance versus Size of Reflection Integration Area . . 190

Figure 97 - Percent of Maximum Target Iluminance versus Size of Reflection Integration

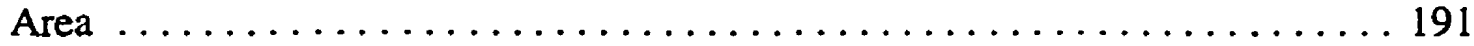

Figure 98 - Contribution of Each Luminaire to Indirect Target nluminance versus Multiple of Mounting Height Distance from Target for Three Spacing to Mounting Height

Ratios .................................. 193

Figure 99 - Design Layout for the Philadelphia Seventh Street Installation . . . . . . 196

Figure 100 - Lighting Layout of Hendersonville Experiment . . . . . . . . . . . 203

Figure 101 - Reflection Grid Layout for Hendersonville Experiment ........... 204

Figure 102 - Roadway Layout for Scottsdale Experiment . . . . . . . . . . . . 207

Figure 103 - Impact of Reflected Light for Single-Sided Arrangement . . . . . . . 213

Figure 104 - Difference of Reflected and Non-Reflected Calculations for Target Contast and Visibility Level for Single Sided Arrangement ............... 214 
Figure 105 - Impact of Reflected Light for Opposite Arrangement $\ldots \ldots \ldots \ldots \ldots 217$

Figure 106 - Difference of Reflected and Non-Reflected Calculations for Target Contrast and Visibility Level for Opposite Arrangement . . . . . . . . . . . 218

Figure 107 - Impact of Reflected Light for Staggered Arrangement . . . . . . . 221

Figure 108 - Difference of Reflected and Non-Reflected Calculations for Target Contrast and Visibility Level for Staggered Arrangement . . . . . . . . . . . 222

Figure 109 - Demonstration of the Visibility of Targets in the Roadway . . . . . . 233

Figure 110 - Visibility Level Demonstration with Glare . . . . . . . . . . . . 234

Figure 111 - Visibility Level Demonstration with Vehicle High Beams . . . . . . 235

Figure 112 - Photograph of Gonioreflectometer . . . . . . . . . . . 240 


\section{Introduction}

In 1934, Waldram discussed the issue of the reflection properties of pavement, why they are important and why they deserve study. He quoted Lord Kelvin, saying:

"When you measure what you are speaking about and express it in numbers, you know something about it; but when you cannot measure it, when you cannot express it in numbers, your knowledge is of a meagre and unsatisfactory kind; it may be the beginning of knowledge, but you have scarcely, in your thoughts advanced to the stage of 'Science', whatever the matter may be."

At that time, the amount of light striking the surface of a roadway, the illuminance, could be calculated but the portion of the incident light which reflects off the pavement into the eye, or the luminance, could not be calculated. This led to the development of road surface reflection studies. The goal of this early pavement reflection research was to develop a system which could estimate the appearance of the road surface. This goal was partially accomplished. Using tables and nomograms, a rough estimate was able to be developed. However, this solution was far from satisfactory; the number of different types of pavements and different installations was too overwhelming. With the development of the computer, the calculation systems were improved and good estimates of road surface luminance were calculated. Still, the different features of the road surfaces had to be classified and estimates had to be made in order to allow the computer 
to handle the volume of data.

At the same time as the development of the improved pavement luminance calculation, studies were performed which related the number of accidents in a given roadway area to lighting criteria. Box [1971] and Box[1973] both state that there is no direct relationship of illumination level to a reduction of accidents, rather a " $U$ " shaped relationship exists, showing that it is possible to both overlight and underlight a roadway. Scott[1980] found no consistent relationship of increasing pavement luminance to a reduction in night versus daytime accidents. Janoff et al.[1977] found an inverse relationship of the visibility index of objects to the nighttime accident rate and a direct relationship of the accident rate to the illumination level. This means that increasing the visibility of objects reduces nighttime accidents and merely increasing the illumination level increases the nighttime accident rate.

The visibility of an object is a measure of how easily things can be seen in the driver's path. Because the relationship to accident rate exists, the interest of roadway lighting designers has changed. They are no longer interested in just the luminance of the pavement surface but in visibility. Design standards are being changed to incorporate this new outlook. The Iluminating Engineering Society of North America (IESNA) has recently proposed a change to the roadway lighting standard to include the visibility of objects rather than strictly luminance. 
The calculation of this new quality criterion has led to some difficulty. The interest in pavement reflection is no longer just the reflection of the light from the pavement surface into the eye of the observer, but it is also the reflection of the light onto objects in the roadway, onto the walls of a tunnel, or into the night sky. The current methods of calculation of the pavement reflection characteristics have become inadequate. The purpose of this research project is to develop new road surface reflection data, as well as to develop a calculation system which will allow for the estimation of target visibility. 


\section{Roadway Lighting Design}

The Mluminating Engineering Society of North America Recommended Practice for Roadway Lighting states that the purpose of roadway lighting "is to produce quick, accurate, and comfortable seeing at night"(IESNA - RP-8[1997] page 2). This will allow roadways to be as useful at night as they are during the day and should reduce accidents, improve security and improve traffic flow. Lighting in the roadway environment comes from four sources, the fixed roadway lighting, the vehicle headlighting, signal and sign lighting, and off-roadway lighting sources. RP-8 [1997] deals only with the fixed lighting design; other standards are available for vehicle headlighting.

RP-8[1997] proposes lighting levels for two categories of roadways. The first is the urban roadway. These are lower-speed city streets where there is high pedestrian usage, many entrances to the roadway and areas adjacent to the roadway like parks and residences. In lighting the urban areas, the headlight is a major contributor to the luminance of objects in the roadway. Light spillage from the roadway in the adjacent areas is a concern. The second roadway type is high-speed roadways, which are usually divided and have controlled access. Lighting for this environment is generally provided by fixed luminaire installations and the area of concern for the driver is close to one hundred metres in front of the vehicle. At this distance of interest, the vehicle headlamps are generally not a contributor to the visibility of the driver as the illumination provided for objects is not substantial. It is this type of roadway where the visibility of targets and the pavement luminance are defined as the quality criterion for the roadway lighting 
design. A pictorial example of the Visibility Level and the influence of glare is provided in Appendix A.

Table 1 shows the specified values of target visibility and pavement luminance in RP$8[1997]$.

Table 1 - Design Criteria for High Speed Roadways Specified in IESNA RP-8[1997] (page 14)

\begin{tabular}{|c|c|c|c|c|c|}
\hline \multirow{2}{*}{$\begin{array}{l}\text { Classification of } \\
\text { Roadway Area }\end{array}$} & \multirow{2}{*}{$\begin{array}{c}\text { STV Criteria } \\
\text { Wtg. Avg. } \\
\text { VL } \\
\text { (STV) }\end{array}$} & \multicolumn{4}{|c|}{ Luminance Criteria } \\
\hline & & $\underset{\substack{\mathrm{L}_{\text {avg }} \\
\text { Median } \\
\mathrm{m}^{2}}}{.}$ & $\underset{\substack{\mathrm{L}_{\mathrm{avg}} \\
\mathrm{cd} / \mathrm{m}^{2} \\
24^{2}<M e d i a n<48^{\prime}}}{ }$ & $\underset{\substack{\mathrm{cd} / \mathrm{m}^{2} \\
\text { Median>48}}}{\mathrm{L}_{\text {and }}}$ & $\begin{array}{l}\text { Ratio } \\
\mathrm{L}_{\max } / \mathrm{L}_{\min }\end{array}$ \\
\hline Freeway "A" & 3.2 & 0.4 & 0.5 & 0.4 & 6 to 1 \\
\hline Freeway "B" & 2.6 & 0.4 & 0.3 & 0.3 & 6 to 1 \\
\hline Expressway & 3.8 & 0.5 & 0.4 & 0.4 & 6 to 1 \\
\hline $\begin{array}{l}\text { Other Roadways } \\
\text { - Undivided }\end{array}$ & 2.6 & 0.5 & - & - & 6 to 1 \\
\hline $\begin{array}{c}\text { Isolated Traffic } \\
\text { Conflict Area }\end{array}$ & 2.6 & 0.5 & 0.4 & 0.4 & 6 to 1 \\
\hline
\end{tabular}

In this table, the target visibility is specified in terms of minimum Small Target Visibility (STV) which is a weighted average of several Visibility Level calculations in a grid on the roadway. The pavement luminance is specified in terms of the average luminance and the uniformity ratio of maximum luminance to minimum luminance. The road classifications are defined by their complexity. Freeway "A" represents a high volume 
visually complex roadway. Freeway "B" represents all other controlled access roadways. Expressways are divided roadways which have partial access control. Other roadways are any undivided roads which have a maximum speed over $72 \mathrm{~km} / \mathrm{hr}$ ( 45 miles $/ \mathrm{hr}$ ). The final roadway type is isolated interchanges where fixed lighting installations are used. The median is the central division of the traffic direction. For the undivided highway, there is no median and thus no specification is made according to the median distance.

The reflection properties of pavement are used to calculate both of these high-speed roadway criteria. The original data describing pavement reflection was developed specifically for the pavement luminance calculation. Again, with the proposed changes in RP-8 [1997], the reflection of light onto other objects has become important and is the area of concern for this research. The calculation systems for both the pavement luminance and the target visibility are described in the following sections.

\subsection{Road Lighting Calculations}

The pavement luminance system presented in this section has been in use in Europe since the mid 1970's and in North America since 1983.

The luminance of a point on a road surface can be calculated from the light intensity of a luminaire directed to it, the geometry of the installation and the reflection characteristics of the pavement. 


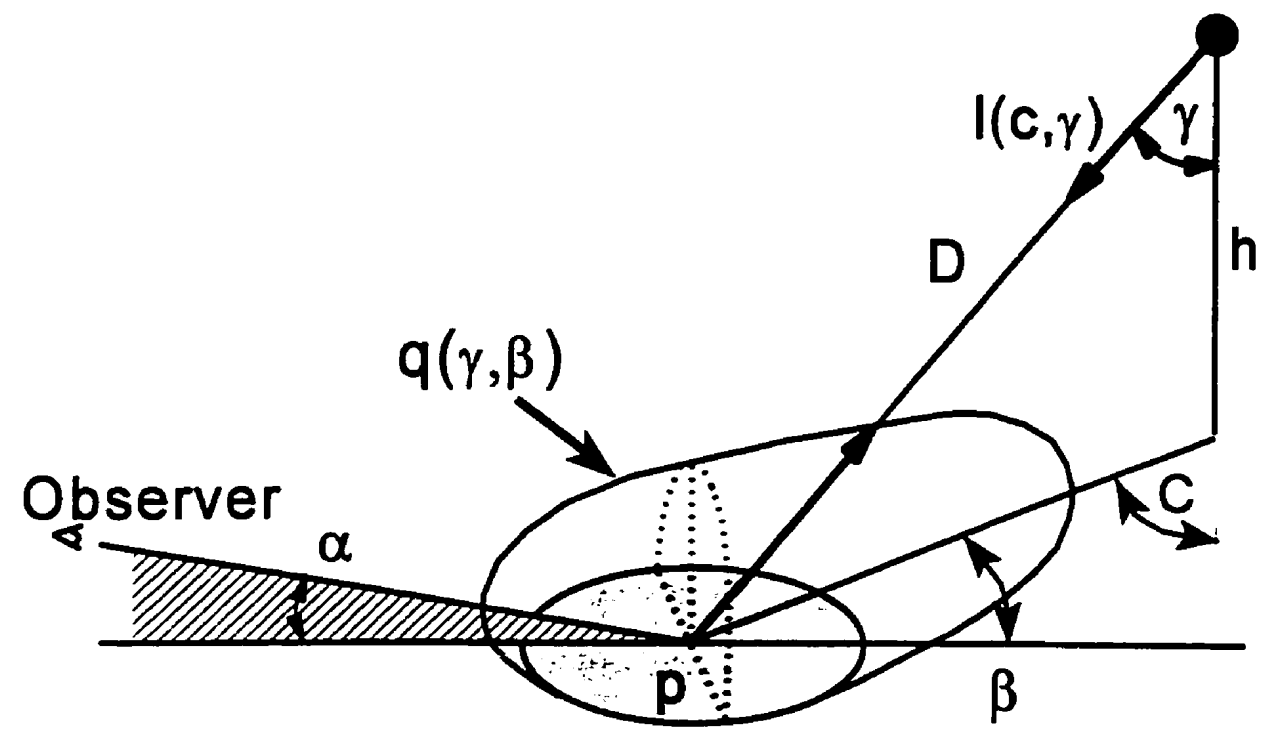

Figure 1 - Geometry for Calculating Pavement Luminance

The geometry of the calculation is shown in Figure 1. The luminance from a single luminaire at the point $P$ is calculated as:

$$
L_{p}=q(\gamma, \beta) \cdot E_{p}
$$

where $E_{p}$ is the horizontal illuminance at point $P$ and $q(\gamma, \beta)$ is the directional reflection property of the pavement. The horizontal illuminance at the point is:

$$
E_{p}=\frac{I(c, \gamma)}{D^{2}} \cdot \cos (\gamma)
$$

where $\mathrm{I}(c, \gamma)$ is the intensity of the light source in the direction defined by the angles $c$ 
and $\gamma$ and $D$ is the distance from the point $P$ to the light source.

D follows from:

$$
D=\frac{h}{\cos (\gamma)}
$$

where $h$ is the mounting height of the luminaire. With the substitution of $D$ into Equation 2 we obtain:

$$
E_{p}=\frac{I(c, \gamma)}{h^{2}} \cdot \cos ^{3}(\gamma)
$$

And Equation 1 reads with $E_{p}$ :

$$
L_{p}=\frac{I(c, \gamma)}{h^{2}} \cdot q(\gamma, \beta) \cdot \cos ^{3}(\gamma)
$$

The reflection properties of the pavement are combined with the cosine into a function, $r$ :

$$
r(\gamma, \beta)=q(\gamma, \beta) \cdot \cos ^{3}(\gamma)
$$

This $r$ function is measured for pavement surfaces and standard tables have been set up for many different luminaire geometries. It was introduced to reduce the magnitude of the values of $q$ and to ease the measurement methodology. 
It is noteworthy that the luminance of the pavement is always relative to a $1^{\circ}$ observation angle $\left(\alpha=1^{\circ}\right.$, Figure 1$)$. The observation angle results from the chosen geometry of $1.45 \mathrm{~m}$ height of the driver's eye looking at a point at 83 meters in the distance. This geometry has been chosen by the IESNA and is specified in RP-8 [1997]. The driver's eye height could be much lower than the $1.45 \mathrm{~m}$ for many vehicles or could be much higher in vehicles such as trucks and buses. The 83 metres is the centre, in perspective, of the field of evaluation ranging from 60 metres to 160 metres from the observer.

Using the reflection tables previously described, the luminance equation reduces to:

$$
L_{p}=\frac{I(c, \gamma)}{h^{2}} \cdot r(\gamma, \beta)
$$

Calculations of the luminance as seen under a $1^{\circ}$ observation angle can be performed for any road lighting installation and geometry and for any point on the pavement surface. For a realistic roadway lighting installation with many luminaires, the total luminance of a point on the roadway surface is the sum of the luminance from each luminaire in the design.

The intensity, $\mathrm{I}(\mathrm{c}, \gamma)$, used in this calculation is found in intensity distribution tables which are provided by the luminaire manufacturer. These tables specify the intensity of the luminaire at varying angles of $\gamma$ and $c$. In the North American caiculation system, the angle measurement $c$ is a rotational angle about the centre of the luminaire. $c=0^{\circ}$ is defined as perpendicular to the street curb in the street side direction. When performing 
the pavement luminance calculation, the intensity distribution table, the mounting height, the overhang of the luminaire from the support pole, the tilt of the luminaire from horizontal, the rotation of the luminaire about its horizontal axis and the orientation of the luminaire to the curb on the roadway must all be recorded. The angles, $c, \gamma$ and $\beta$, of the luminaire and the point of interest are then calculated and modified to account for the tilt, rotation and orientation of the luminaire. These angles are then used to look up the appropriate intensity in the distribution table. The intensity is linearly interpolated between the two nearest entries in the table, or for greater accuracy a quadratic interpolation can be used. These intensity tables are measured such that the linear interpolation between two points results in less than $5 \%$ error.

As described earlier, the quality criterion used for roadway lighting design in this pavement luminance system are the average luminance and the uniformity ratio of the maximum luminance to the minimum luminance. In order to calculate these criteria, a grid of several points on the road surface is used. The pavement luminance of each of the points on this grid is calculated and the quality criterion are then calculated based on this grid of points. The process of designing a roadway based on these criteria is an iterative one. The initial design is proposed by the lighting engineer and the criterion are then calculated. Based on these results, the design is modified and the calculation is performed again. This process will continue until the criterion specified in RP-8 are met. There are other aspects of the roadway lighting design which come into play in this methodology. The IESNA also specfies the veiling luminance, which is a determination 
of the disability glare in the installation, must be less than 0.3 of the pavement luminance. The visual flow of the luminares following the road path, and the stray light from the installation onto the surrounding areas are all a concern. A full description of these other criterion are defined in RP-8 [1997].

The intemational requirements for roadway lighting specified by the Commission Internationale d'Eclairage (CIE) Report 30-2, [1982], are different than those specified by RP-8 [1997]. Similarly, the definition of the angles and the calculation methodology are different between these two specifying bodies. The overall impact of the differences in the calculation methods is generally considered to not be significant. Throughout this research, the IESNA methodology is used.

\subsection{Target Luminance and the Visibility Level}

As discussed, the IESNA proposed system for evaluating the quality of a road lighting installation is the Small Target Visibility (STV). The STV is calculated based on the visibility level (VL) of objects on the pavement surface. The VL is a measure of the driver's ability to see objects in the roadway and is based on the visibility of a critical detail which represents the smallest object which must be seen by the driver for safe travel. The current target used in the calculation is a flat square $18 \mathrm{~cm}$ by $18 \mathrm{~cm}$ placed with its vertical face facing the driver and a reflectance of approximately $20 \%$. This detail might represent a lost muffler or a small obstacle in the driver's path. The $20 \%$ target reflectance was chosen by the IESNA to represent a worst case scenario for objects 
in the roadway. If an actual object was more reflectant than $20 \%$ it would be more easily seen than the calculated object. Some discussion has been put forward for using a 50\% reflectance target, which might more closely represent physical objects. Mace [1997] has found that STV calculated with a 50\% target seems to be no more closely related to accident rates than STV calculated with a $20 \%$ target.

Lecocq [1993] suggests a multifaceted hemispherical target as an alternative to the flat target as a quality criterion. This target type represents realistic objects more closely than the flat target. These results have been futher investigated and are presented in Lecocq [1997] and King [1997]. In both of these investigations, it was found that the multifaceted targets is more visible and more recognizable that the flat target. These investigations also show a strong relationship of the calculated visibility level to the actual visibility. The advantage of the multifacted target over the flat target is that the flat target is oriented only towards vertical illuminance where the multifaceted target is able to reflect light received from all directions. These results show that the flat target does represent a worst case in the visibility calculation. The current revision of RP-8 continues to use the flat target as the quality criterion and that calculation will be used in this research.

The $\mathrm{VL}$ is the ratio of the actual target contrast to the target's threshold contrast. The visibility level calculation is: 


$$
V L=\frac{C_{a c t}}{C_{t h}}=\frac{\frac{L_{\text {Target }}-L_{\text {Background }}}{L_{\text {Background }}}}{C_{t h}}
$$

where $\mathrm{L}_{\text {Target }}$ is the luminance of the target face and $\mathrm{L}_{\mathrm{Background}}$ is the luminance of the pavement around the target. The threshold contrast, $\mathrm{C}_{\mathrm{th}}$, is the minimum contrast of the target luminance to the background luminance which allows the target to just be seen. $\Delta \mathrm{L}_{\mathrm{h}}$ is the threshold luminance difference and is related to the threshold contrast as:

$$
C_{t h}=\frac{\Delta L_{t h}}{L_{\text {Background }}}
$$

$\Delta \mathrm{L}_{\mathrm{h}}$ depends on the size of the target, the age of the observer, the amount of time the driver has to see the target and the background luminance behind the target. The threshold contrast is calculated by an algorithm developed by Adrian [1989] which is shown in Appendix B.

In the calculation of VL, the pavement or background luminance is calculated in the same manner as previously described. For the target, the background luminance is the average of the pavement luminance just over the top centre of the target and at the bottom centre of the target. The target luminance caiculation however requires special consideration.

The luminance of a target on a road surface is a result of two components. The first is the 
direct light component from the luminaire itself and the second is the component reflected off the pavement surface (Figure 2).

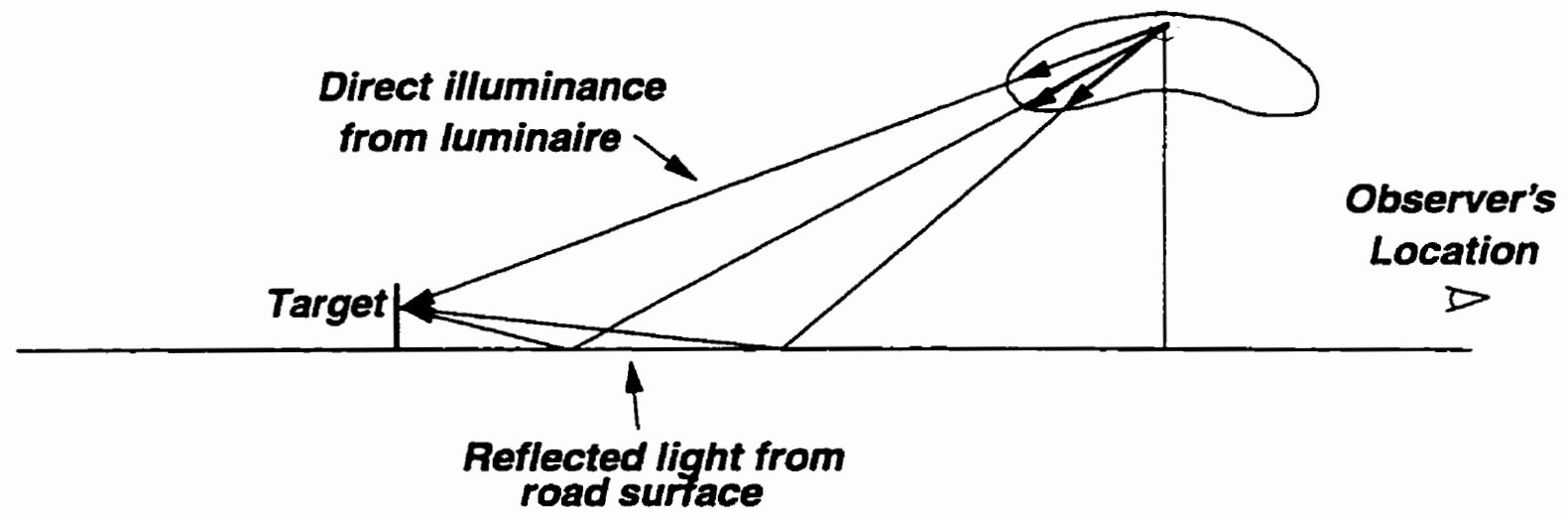

Figure 2 - Sources of Target Luminance

The overall luminance of the target, as seen by the Observer, can be calculated as:

$$
L_{T}=\frac{E_{T \nu} \cdot \rho}{\pi}
$$

where $E_{T v}$ is the vertical illuminance of the target and $\rho$ is the reflectance of the target surface. The vertical illuminance of the target, $E_{T v}$ is then composed of two parts:

$$
E_{T v}=E_{T R}+E_{T D}
$$

where $E_{T D}$ is the direct component and $E_{T R}$ is the reflected component. $E_{T D}$ is easily calculated: 


$$
E_{T D}=\frac{I(c, \gamma)}{h^{2}} \cdot \cos (\gamma) \cdot \cos (\beta) \cdot \cos ^{2}(\gamma)
$$

The reflected portion $E_{T R}$ is more difficult. The geometry of the reflected portion is shown in Figure 3.

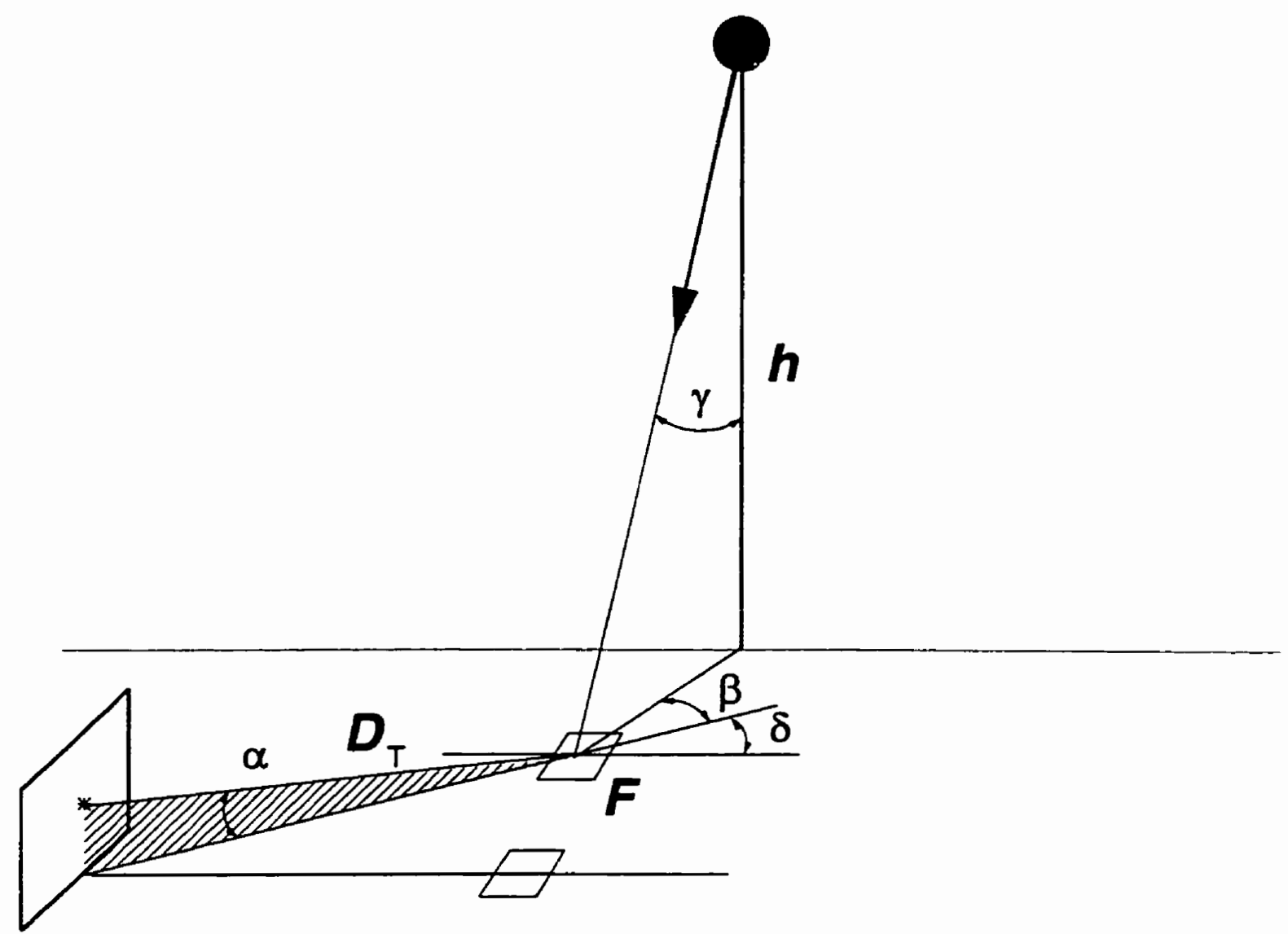

Figure 3 - Geometry for the Indirect Target Dluminance Calculation

If you consider a small portion of the road surface as a light emitting source, then the vertical illuminance of the target would be: 


$$
E_{T R}=\frac{I_{s}}{D_{T}^{2}} \cdot \cos (\alpha) \cdot \cos (\delta)
$$

where $I_{s}$ is the intensity emitted from the road surface area $(F), D_{T}$ is the distance from the pavement portion to the target, $\alpha$ is the angle of the reflected light upward, and $\delta$ is the angle of the reflected light to the normal of the target surface.

The intensity of the light coming from the portion of the pavement, $L$. would be:

$$
I_{s}=L_{p} \cdot F \cdot \sin (\alpha)
$$

where $L_{p}$ is the luminance of the pavement and $F$ is the area of the pavement portion. This means that the reflected portion of the target illuminance is calculated as:

$$
E_{T R}=\frac{L_{p} \cdot F \cdot \sin (\alpha)}{D_{T}^{2}} \cdot \cos (\alpha) \cdot \cos (\delta)
$$

This calculation can be performed relatively easily except for one component. The pavement reflectance measurements are always based on a $\alpha=1^{\circ}$ observation angle. However, the target and the pavement reflection points show a different geometry with variable angles for $\alpha$. A new factor must be incorporated to the pavement reflection calculations, one which takes the influence of the change in observation angle into account. This would result in the pavement luminance calculation depending on three variables: 


$$
L_{p}=\frac{I(c, \gamma)}{h^{2}} \cdot r(\alpha, \gamma, \beta)
$$

Like the pavement luminance criteria, the overall target luminance is calculated based on the contribution of all of the luminaires in the design.

The STV as the quality criterion is based, like the pavement luminance, on the average of a calculation of a grid of points on the pavement surface where the target is mathematically placed and its visibility is calculated. The STV is calculated from this grid as a weighted average using the following methodology :

1) The visibility level at all of the grid points is converted to a factor called RWVL. This factor is calculated as:

$$
R W V L=10^{-.1 \cdot|V L|}
$$

2) The RWVL values for each of the points is averaged

3) The RWVL is converted to a weighted average using the formula:

Wtg. Avg. $V L=-10 \cdot \log _{10}($ Average of $R W V L)$

It is noteworthy that the current revision of RP-8[1997] specifies the calculation of the direct illuminance of the target only. The indirect contribution is not calculated. The calculation methodology of the indirect target luminance and the relationship of the pavement luminance to observation angle is not well known and is the purpose of this research. 


\section{Reflection Properties of Pavement Surfaces}

The reflection of light from surfaces can be a result of many different reflection modes: specular, spread, diffuse and compound. The nature of the reflection depends on the nature of the surface of the reflecting material and on the geometry of the reflected light

source. A surface can be specular at one geometry and diffuse at another. Pavement has a character of reflection which depends on several different aspects of the surface, and makes it difficult to predict the reflection characteristic. Due to this complexity, different classification systems of pavement have been proposed. These systems are an attempt to allow the lighting designer to identify the reflection properties of the surface based on only a few criteria.

\subsection{Reflection Modes}

The reflection modes define the mathematics of calculating the brightness of a surface. The extreme modes of reflection, specular and diffuse have very simple mathematical relationships. The other modes are difficult to represent. These are defined by the IESNA Handbook [1984].

\subsubsection{Specular Reflection}

Specular reflection is the first extreme reflection mode. It is evident in polished surfaces such as mirrors. The nature of the reflection is such that the angle of the incident ray to the normal of the surface is equal to the angle of the reflected ray to the normal of the surface as in Figure 4. 


\section{Figure 4 - Specular Reflection Profile}

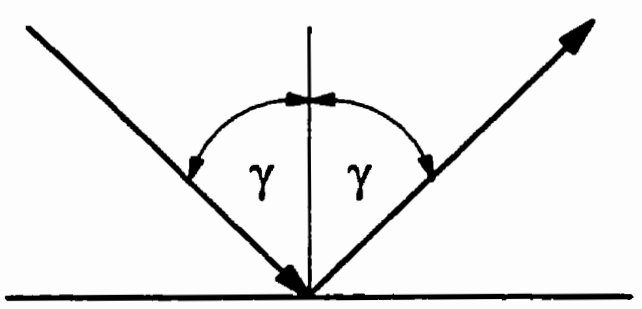

In perfect reflection, the intensity of the reflected beam is equal to the intensity of the incident beam. The reflection is seldom perfect however, and a simple scalar is used for the intensity. This scalar represents the reflectivity of the surface and is noted as $\rho$. The mathematical relationship is then:

$$
\begin{gathered}
I_{\text {incudent }}=\rho \cdot I_{\text {reflected }} \\
\text { and } \\
\gamma_{\text {incident }}=\gamma_{\text {reflecied }}
\end{gathered}
$$

This relationship is well known and is the basis of many optics and imaging technology calculations.

\subsubsection{Spread Reflection}

Spread reflection is similar to specular reflection except that instead of a single ray of reflected light, the reflection occurs throughout a cone of reflected rays as shown in Figure 5. This is usually a result of figuring in the surface such as brushing, etching or peening. 


\section{Figure 5 - Spread Reflection Profile}

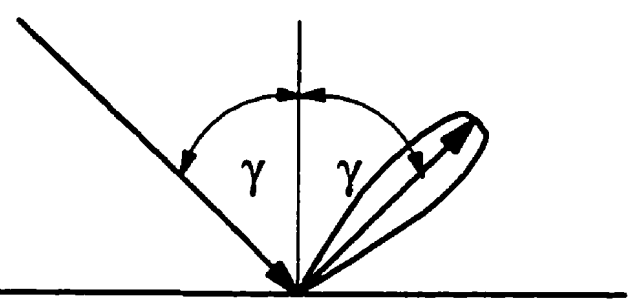

The mathematics of this relationship are similar to specular reflection in that the angle of the centre of the reflected cone is equal and opposite to the incident angle and that the spatial integration of the intensities in the reflected cone is equal to the incident intensity multiplied by the reflectivity of the surface:

$$
\begin{gathered}
I_{\text {incident }}=\rho \int_{\text {conee }} I_{\text {reflected }} d I \\
\text { and } \\
\gamma_{\text {incident }}=\gamma_{\text {reflected (centre of cone) }}
\end{gathered}
$$

Spread reflection is generally used for visual effects such as highlighting, sparkling and starbursting of reflected images.

\subsubsection{Diffuse Reflection}

Diffuse or Lambertian reflection is the cther extreme of reflection from specular reflection. Rough surfaces are generally Lambertian. A perfectly diffuse surface reflects equal brightness in all directions independent of the angle of observation. The term Lambertian comes from Lambert's cosine law which states that the intensity of the reflected light from a perfectly diffusing surface varies only with the cosine of the angle between the observation angle and the normal of the surface. For Lambert's cosine law, 
the incident intensity is always considered perpendicular to the surface, $\mathrm{I}_{0}$. Lambert's cosine law is represented by the following equation:

$$
I_{\text {reflected }}=\rho \cdot I_{o} \cdot \cos (\theta)
$$

where $\rho$ is the reflectivity of the surface. The result is shown in Figure 6.

Figure 6 - Diffuse Reflection Profile

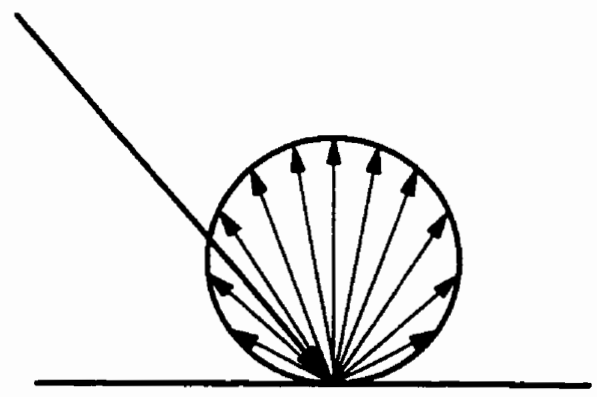

Luminance is calculated as the quotient of the intensity in a given direction coming from a surface and the projected area of the surface, as shown in Figure 7 and Equation 22:

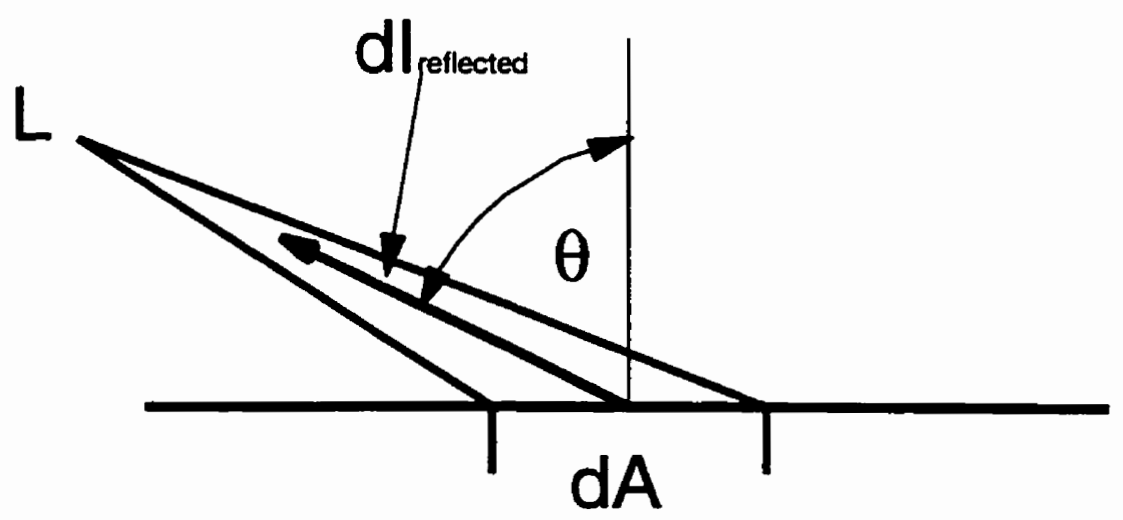

Figure 7 - Relationship of Luminance to Intensity 


$$
L=\frac{d I_{\text {reflected }}}{d A \cdot \cos (\theta)}
$$

Using this relationship, and Lambert's cosine law, it can be seen that:

$$
L=\rho \cdot \frac{d I_{o}}{d A}
$$

This relationship shows that the luminance of the surface is independent of the angle of observation. Using the geometry and nomenclature assigned for pavement surfaces, this relationship means that the luminance of the surface is independent of $\alpha$ and $\beta$ and only depends on $\gamma$ since:

$$
I_{o}=I_{\text {luminatre }} \cdot \cos (\gamma)
$$

Lambertian reflection provides a simple method to calculate the luminance of a surface based on the incident intensity. Many rough surfaces can be approximated as Lambertian surfaces.

\subsubsection{Compound Reflection}

Most surfaces actually have a compound or mixed reflection nature. This means that, depending on the angles of observation and of incidence, the mechanism at work can be either diffuse reflection, specular reflection or spread reflection as shown in Figure 8. 


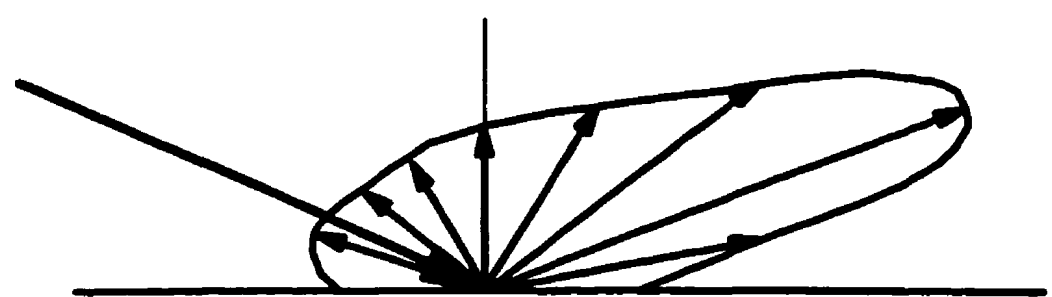

Figure 8 - Compound Reflection Profile

There is no easy definition of the reflection intensities for compound reflection surfaces.

For these surface types, the concept of the bidirectional reflectance distribution function (BRDF) has been developed. The BRDF is the ratio of the differential luminance of a ray in a given direction to the differential luminous flux density incident from another given direction [IESNA, 1984]. In terms of roadway lighting applications, the reflection function $r$ is related to the $B R D F$ through equation :

$$
L_{p}=E_{p} \cdot \frac{r(\alpha, \beta, \gamma)}{\cos ^{3}(\gamma)}
$$

Therefore,

$$
\frac{r(\alpha, \gamma, \beta)}{\cos ^{3}(\gamma)}=\frac{L_{p}}{E_{p}}=B R D F=q(\alpha, \beta, \gamma)
$$

The BRDF function is dependent on the angle of incidence of the light and the angle of observation. The geometry of the BRDF is shown in Figure 9. 


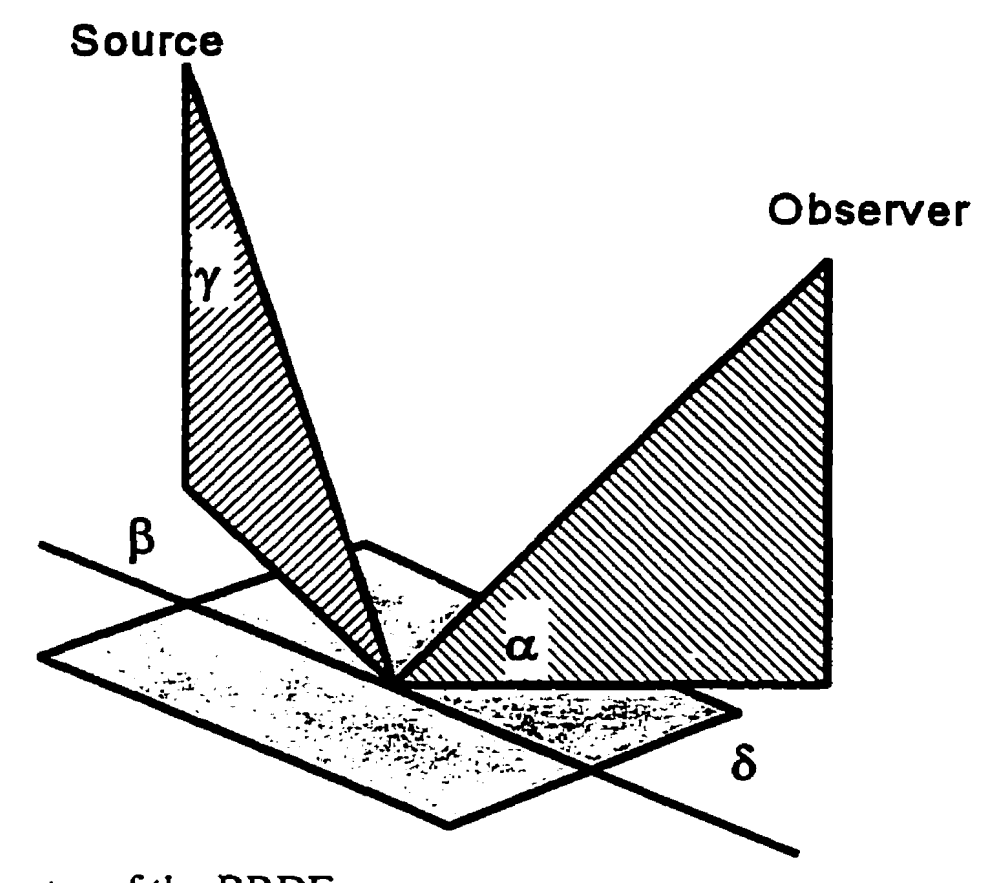

Figure 9 - Geometry of the BRDF

The CIE has determined that for roadway lighting calculations, the influence of the angle $\delta$ can be ignored. This will be investigated briefly as part of the results of this experiment.

The has been some work to quantify the nature of the BRDF according to the physical make up of the surface itself. This work, generally performed by Beckmann and Spizzichino[1963] for the radio industry, uses diffraction laws to calculate the nature of the surface reflection. This is discussed later as a possible analytical solution of the reflection properties.

\subsection{Pavement Reflection}

Research has shown that the reflection properties of pavement surfaces depend on the 
colour, texture, and structure of the surface (Jung et al.[1984]). Some factors which influence these parameters are the materials chosen for the surface, the pavement recipe, and surface wear.

Pavement is a mixture of aggregate stone, usually over $75 \%$ by volume, and a binder material to hold the aggregate in the road surface. The binders used are generally either Portland cement or asphalt. The type of aggregate used in pavement varies greatly. Usually, the aggregate is stone from the local area, used to minimize trucking and transport costs. Some artificial aggregates are used to provide a better skid resistance or a better light reflection. The light reflection properties of the pavement surface vary greatly with the type of material used. If the pavement has a concrete binder, the reflectance is approximateiy $10 \%$. For an asphalt surface the reflectance is about $5 \%$ (Ketvirtis and Bastianpillai [1978]). However, if a light colour aggregate is used in the asphalt, the reflectance can be as high as $15 \%$. The goal of the road specifier is to choose a material recipe which incorporates good skid resistance, good reflection properties and cost effectiveness.

As stated, the mode of reflectance of the pavement is compound. The reflection of light off of the pavement surface is generally considered to be a result of several different facets of the material in the pavement. These facets are random in size, shape and facing direction. Some of these facets reflect light specularly, some reflect light diffusely, some absorb light and some are in shadow. This aspect of reflection is shown in Figure 10. 

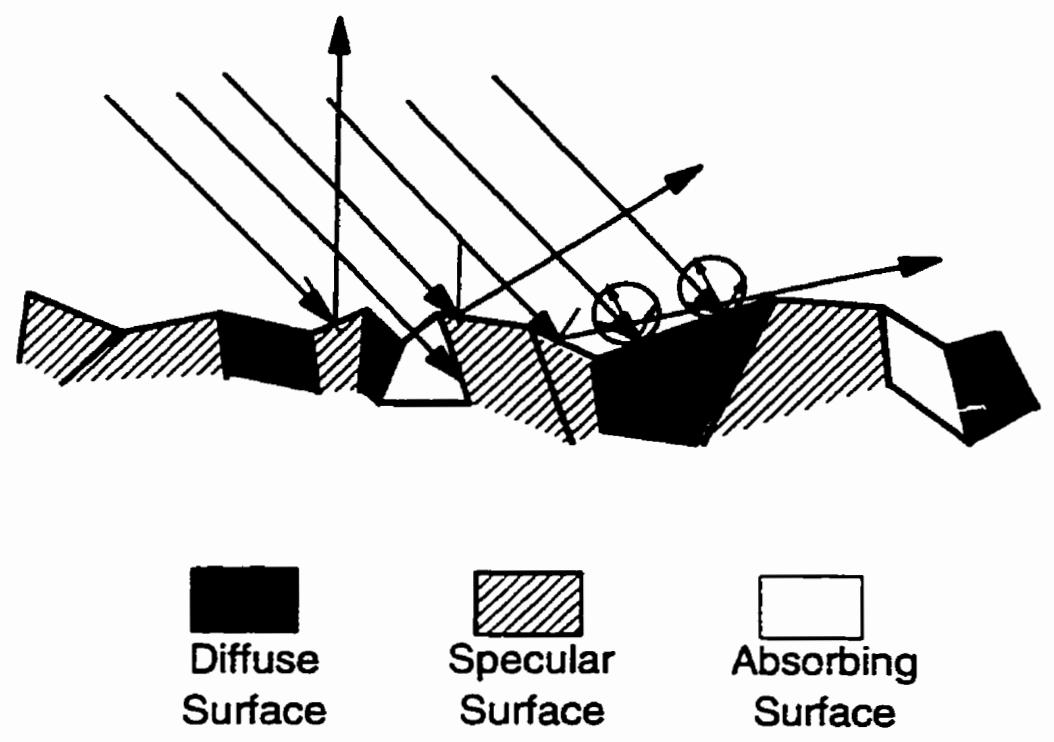

Figure 10 - Contribution of Pavement Facets to the Reflection Profile

The facing angle of the various facets also changes the local angle of incidence and reflection as shown in Figure 11. This also contributes to the reflection characteristic of the pavement.

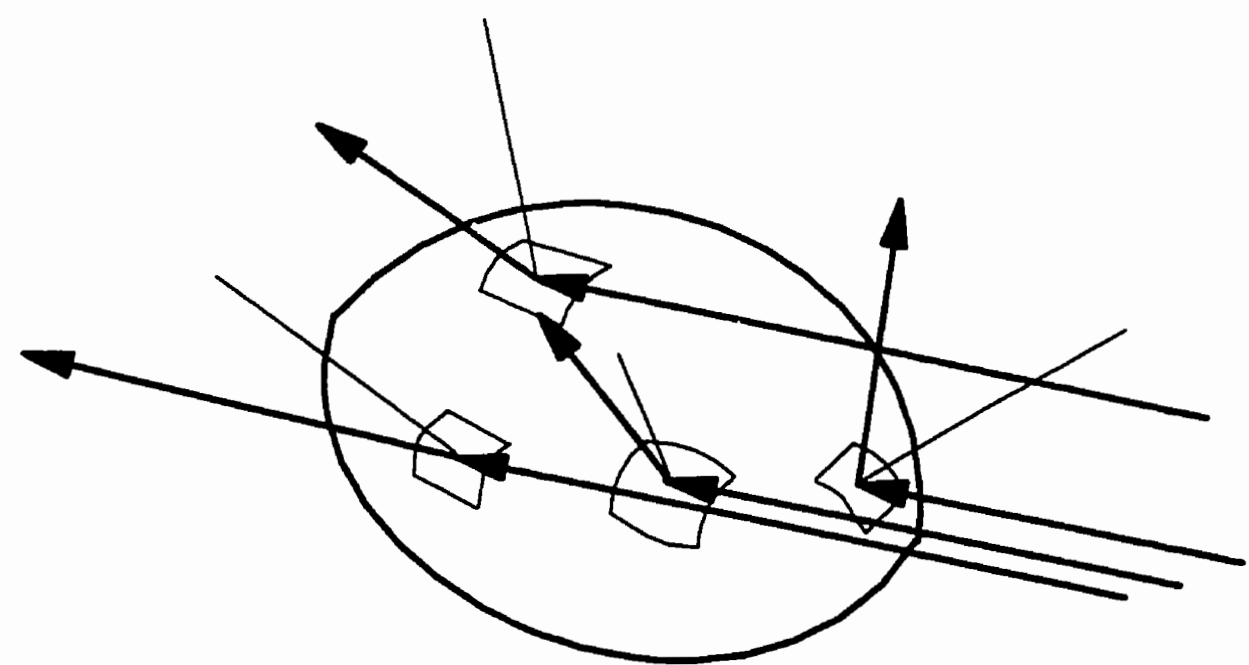

Figure 11 - Local Reflection and Incidence Angles from a Single Stone in the Pavement Surface from CIE [1995] 
The final aspect of the pavement facets which contributes to the reflection characteristics is surface roughness. Each of the various facets in pavement surface has a roughness inherent in the stone itself referred to as micro roughness. Micro roughness is generally so small that it is not perceivable by eye. The other type of surface roughness is macro roughness. This is perceivable by eye and is generally related to the size of the stone aggregate. These roughness types are shown in Figure 12.

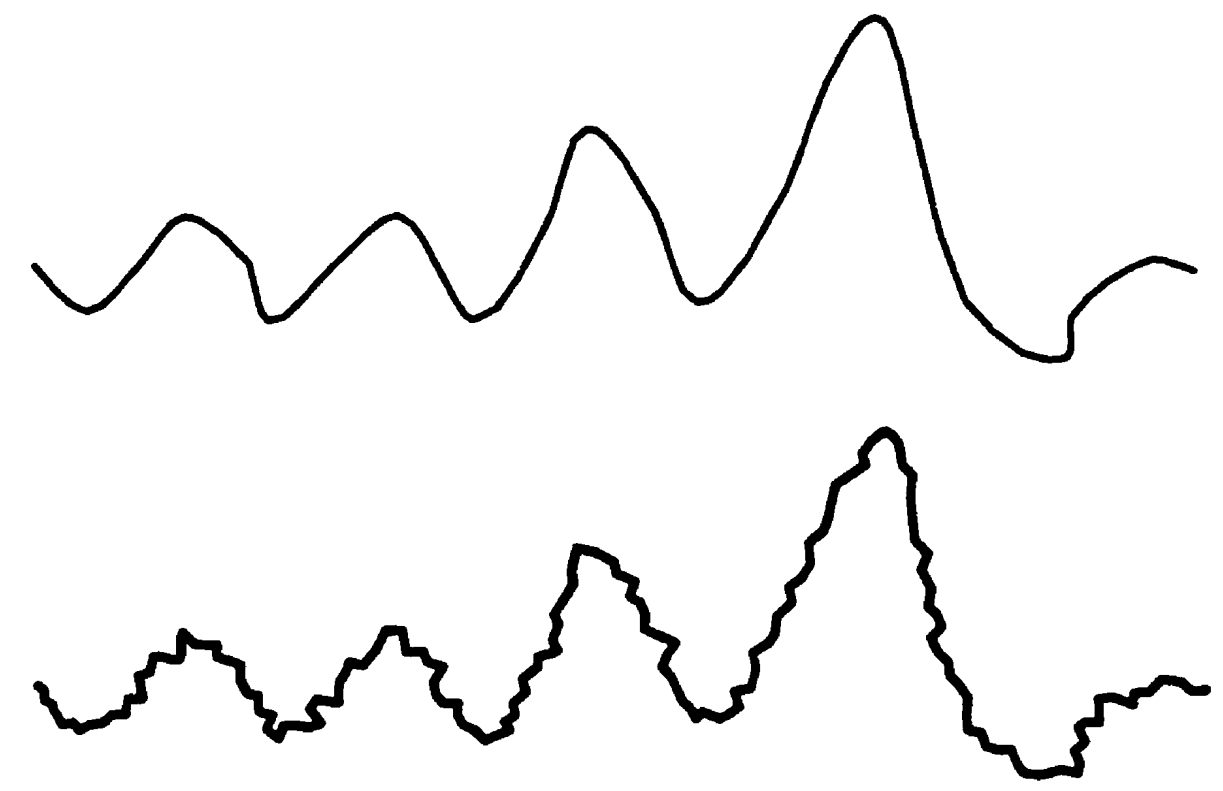

Figure 12 - Macro Roughness (Top Diagram) and Micro Roughness (Bottom Diagram)

The interesting feature of this roughness model is the interaction with wet surfaces. When the pavement is dry, both of the roughness natures of the pavement contribute to the reflection. When the surface is wet, the water will fill the micro roughnesses of the surface leaving the macro roughness to be the dominant contributor. The wet pavement becomes much more specular. This would lead to the assumption that the micro roughness contributes to the diffuse nature of the reflection and the macro roughness 
contributes to the specular portion of the reflection. This has yet to be shown conclusively in any research. Some attempts have been made to describe the reflection properties of pavement surfaces in terms of the surface roughness. Some of these procedures will be reviewed later.

Schmidt-Clausen and Van Bommel[1972] have studied the polarization character of pavement surfaces. They found that when using polarized headlamps, the luminance of the pavement was less and the luminance of objects in the roadway was greater than for the same lighting geometry with non-polarized light. This result shows that pavement surfaces behave reasonably well in a polarized environment. Our interests are in studying non-polarized light as we are considering fixed lighting installations.

As mentioned, the reflection characteristics of the pavement have been measured for hundreds of different pavement types since the mid 1960's. These measurements have mostly been carried out under a $1^{\circ}$ observation angle. In an effort to simplify the lighting design system, road classes and pavement classification systems were developed. These systems use criteria to separate the surfaces into classes which are generalizations of all the pavement measurements and which represent roads with similar reflection characteristics. A generalized table of reflection data, referred to as an r-table is used for each road class. The results of the current experiment will be evaluated in terms of the various classifications systems. 


\subsubsection{CIE Road Surface Classification}

The current system used in the CIE for road classification is based on three criteria.

These criteria are:

\section{$q_{0} \quad$ Average Luminance Coefficient \\ S1 and S2 Specular Factors}

The first is an average luminance coefficient. The component called $q_{0}$ is calculated as the integral of the product of the reflection factor $q$ and the solid angle represented by $q$ divided by the solid angle of all of the measurements. Equation 27 shows the calculation of $\mathrm{q}_{0}$.

$$
q_{0}=\frac{\int_{\Omega} q \cdot d \Omega}{\int_{\Omega e} d \Omega}
$$

$\Omega e$ is the solid angle of the integration area defined by the CIE Report 30-2 [1982]. The integration limits for the $q_{0}$ calculation are $\beta=0^{\circ}$ to $180^{\circ}$ and $\tan (\gamma)=-4$ to 12 . The value, $q_{0}$, is just a scaling factor of the overall brightness of the surface and does not change the overall shape of the reflection characteristic.

The other factors, $\mathrm{S} 1$ and $\mathrm{S} 2$, are used to define the road class. $\mathrm{S} 1$ is the ratio of an $\mathrm{r}$ value which is generally large for specular reflection to a factor which is generally large for diffuse reflection. Thus $\mathrm{S} 1$ is a measure of the degree of specular reflection. 
Similarly, $\mathrm{S} 2$ is the ratio of the average luminance coefficient to a value which is large for diffuse reflection. These factors are shown in Equations 28 and 29.

$$
S 1=\frac{r(0,2)}{r(0,0)}=\frac{r\left(\beta=0^{\circ}, \tan (\gamma)=2\right)}{r\left(\beta=0^{\circ}, \tan (\gamma)=0\right)}
$$

and

$$
S 2=\frac{q_{0}}{r(0,0)}
$$

Several atlases of reflection data have been published in order to assist the roadway designer. The atlas for Canadian pavement surfaces was developed by the University of Toronto in 1983 (Dmitrevsky and Bassett [1983]). Using the three reflection criteria, the lighting designer could choose a pavement reflection table from the atlas which most closely matches the pavement of interest. The chosen r-table would then be used for the design.

A simpler method than atlases is to classify pavement surfaces into Road Classes. Using the $S 1$ value, the road surface reflections were divided into the four classes, R1, R2, R3 and $\mathrm{R} 4$. The classes are arranged as $\mathrm{R} 1$ to $\mathrm{R} 4$ from the flatest to the most specular. The limits of the SI values for the road classes are shown in Table 2. 
Table 2 - Road Classification Criteria Boundaries (CIE 30-2 [1982])

\begin{tabular}{||c|c|c|c|c||}
\hline \hline Road Class & S1 Limit & S1 of Standard & S2 of Standard & Normalized $\mathrm{q}_{0}$ \\
\hline $\mathrm{R} 1$ & $\mathrm{~S} 1<0.42$ & .25 & 1.53 & 0.10 \\
\hline $\mathrm{R} 2$ & $0.42<=\mathrm{S} 1<0.85$ & 0.58 & 1.80 & .07 \\
\hline $\mathrm{R} 3$ & $0.85<=\mathrm{S} 1<1.35$ & 1.11 & 2.38 & .07 \\
\hline $\mathrm{R} 4$ & $1.35<=\mathrm{S} 1$ & 1.55 & 3.03 & .08 \\
\hline
\end{tabular}

Each road class has a standard table of reflection properties, an r-table, which allows for the calculation of pavement luminance. These tables are the basis of roadway lighting calculations and have standard $\mathrm{S} 1, \mathrm{~S} 2$ and $\mathrm{q}_{0}$ values.

The road classes relate to pavement types as shown in Table 3 . 
Table 3 - Pavement Surface Classifications according to IESNA RP-8 [1997] page 10

\begin{tabular}{|l|l|l|l||}
\hline Class & $q_{0}$ & Description & $\begin{array}{l}\text { Reflection } \\
\text { Mode }\end{array}$ \\
\hline R1 & 0.10 & $\begin{array}{l}\text { Portland cement concrete road surface. Asphalt } \\
\text { road surface with minimum of 12 percent of the } \\
\text { aggregates composed of artificial brightener } \\
\text { (e.g. Synopal) aggregates (e.g. labradorite, } \\
\text { quartzite). }\end{array}$ & Mostly Diffuse \\
\hline R2 & 0.07 & $\begin{array}{l}\text { Asphalt road surface with an aggregate } \\
\text { composed of a minimum 60 percent gravel } \\
\text { (size greater than 10 mm). } \\
\text { Asphalt road surface with 10 to 15 percent } \\
\text { artificial brightener in aggregate mix (Not } \\
\text { normally used in North America). }\end{array}$ & $\begin{array}{l}\text { Compound } \\
\text { (Diffuse and } \\
\text { Specular) }\end{array}$ \\
\hline R3 & 0.07 & $\begin{array}{l}\text { Asphalt road surface (regular and carpet seal) } \\
\text { with dark aggregates (e.g. trap rock, blast } \\
\text { furnace slag); rough texture after some months } \\
\text { of use (typical for most highways). }\end{array}$ & $\begin{array}{l}\text { Slightly } \\
\text { Specular }\end{array}$ \\
\hline R4 & 0.08 & Asphalt road surface with very smooth texture. & Mostly Specular \\
\hline
\end{tabular}

By generalizing road surfaces into classes, an error is inherently introduced into the pavement luminance calculation. A surface might have an $S 1$ value of .43 , which would place it into an $\mathrm{R} 2$ category but which is also close to $\mathrm{R} 1$. This pavement might be considered an R 1.5 class. The error resulting from this generalization was calculated for the pavement luminance. The error parameter was considered for 44 light distributions and 113 pavement surfaces, resulting in 4972 luminance calculations. The resulting error is shown in Table 4. 
Table 4 - Errors Resulting from the Classification of Pavement Surfaces from Van Bommel and de Boer [1980]

\begin{tabular}{|c|c|}
\hline \hline Road Class & Error in (\%) of Average Pavement Luminance \\
\hline R1 & 5.4 \\
\hline R2 & 6.0 \\
\hline R3 & 5.7 \\
\hline R4 & 5.4 \\
\hline
\end{tabular}

This error represents a calculation error only and does not compare road installation measurements to calculations.

\subsubsection{Proposed CIE Classification System}

A new proposal has been suggested for the CIE system. This new system, proposed by the CIE working group TC 4-25, uses the same specular factor, S1, as the current CIE system but recommends a change to the luminance criteria. This proposal is documented in CIE [1995].

The new luminance criteria, $Q_{d}$, is based on diffuse illumination. $Q_{d}$ is the quotient of the luminance of a surface in a given direction based on the illuminance of the surface. $\mathrm{Q}_{\mathrm{d}}$ is calculated in Equation 30 .

$$
Q_{d}=\frac{L}{E}
$$

where 


$$
\begin{aligned}
& L=L_{0} \cdot \sum q \cdot \cos (\gamma) \cdot \Delta \omega \\
& \text { and } \\
& E=L_{0} \cdot \sum \cos (\gamma) \cdot \Delta \omega
\end{aligned}
$$

$L_{0}$ is the luminance of an spheriod enclosure which is located on the pavement surface of interest; $\Delta \omega$ is the solid angle attributed to each $q$ value. $Q_{d}$ replaces $q_{0}$ as it is more easily measured in situ and is supposed to be more closely representative of the lightness of the surface. The S1 factor was not replaced and the S2 factor is not used in this proposed procedure.

There has been extensive work performed in comparing the use of $q_{0}$ and $Q_{d}$ but there has been little acceptance of the new proposal. The draft of the CIE document remains in discussion due to the controversy over the comparative use of these two criteria.

\subsubsection{The $\kappa$ System}

The system which was the basis of the CIE system is a two criteria system which uses $q_{0}$ and a reflection factor $k$. Westermann [1963] developed this factor which uses the relationship of the diffuse portion reflection to the overall luminance coefficient. The factor is calculated as in Equation 32. The input variables are shown in Figure 13.

$$
\kappa=\log \frac{q_{0}}{q_{\min }}
$$




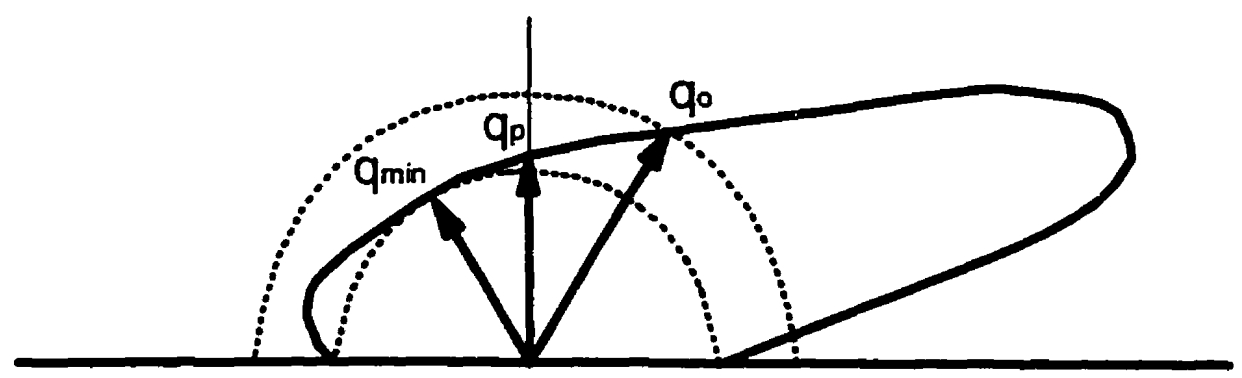

Figure 13 - The Reflection Profile and the Variables Used for the $\kappa$ Calculation

Since finding $\mathrm{q}_{\min }$ requires the measurement of the entire sample, the $x$ system was modified to the $k_{p}$ factor. The $k_{p}$ factor uses the vertical $q_{p}$ value as the denominator rather than $q_{\min }$ as shown in Equation 33.

$$
\kappa_{p}=\log \frac{q_{0}}{q_{p}}
$$

The relationship of $k$ to $\kappa_{p}$ is a simple multiplier.

A five $\mathrm{R}$-Class system was then used based on the $\mathrm{k}$ value as shown in Table 5 .

Table 5 - Classification Boundaries for the $\mathrm{k}$ System (Adrian and Enzmann [1971])

\begin{tabular}{|c|c|c|c|c|}
\hline \hline R1 & R2 & R3 & R4 & R5 \\
\hline$\kappa_{p} \leq 0.22$ & $0.22<\kappa_{p} \leq 0.33$ & $0.33<\kappa_{p} \leq 0.44$ & $0.44<\kappa_{p} \leq 0.55$ & $\kappa_{p}>0.55$ \\
\hline
\end{tabular}

This system provided a method for identifying the pavement surface with only two criteria with the ease of measuring a pavement surface in situ which is not a feature of the current CIE system. It was found that using a two factor system to describe the pavement reflection character was not suitable, so the current three factor CIE system 
was proposed and accepted.

\subsubsection{Alcade System}

The Alcade system was developed at the Laboratory of Acoustics and Lighting in Argentina and is described in Alcade et al. [1996]. This system uses three factors to represent the specularity of the surface. These factors are called $A_{D}, A_{11}$ and $A_{12}$. This system also uses the specularity and the rugosity or roughness of the surface to determine the light reflection properties of the surface. The system began by evaluating the roughness of the surfaces in terms of micro and macro roughness. Using this roughness evaluation, representative values were developed to describe the system. These criteria are as follows:

$$
\begin{array}{ll}
r_{A}=r(0,0) & \text { - Base Condition } \\
r_{A 0}=r(0,3) & \text { - Coefficient for micro roughness } \\
r_{A 1}=r(90,1) & \text { - Coefficient for macro roughness } \\
r_{A z}=r(0,0.625) & \text { - Coefficient for fine macro roughness }
\end{array}
$$

These coefficients are combined into the pavement surface criteria. These criteria are calculated as:

$$
\begin{aligned}
& A_{D}=\frac{r_{A}}{r_{A 0}} \\
& A_{11}=\frac{r_{A I}}{r_{A}}
\end{aligned}
$$




$$
A_{12}=\frac{r_{A 2}}{r_{A}}
$$

$A_{D}$ represents the diffuse reflection which is caused by the micro roughness, $A_{I 1}$ represents the reflection caused by the gross macro roughness and $A_{12}$ represents the reflection caused by the fine macro roughness.

These criteria determine the width and length of the reflection profile. The criteria $A_{D}$ and $A_{11}$ are similar in determining the length of the profile. $A_{12}$ determines the width.

Alcade has determined that this system has a better correlation to the actual pavement reflection profile than any other system. 


\section{Experimental Design}

The main body of this research is the analysis of the reflection properties of pavement surfaces towards the goal of calculating target brightness and target visibility. As stated, the reflection properties are known for a $1^{\circ}$ observation angle, but data is required for a wide range of observation angles. Using pavement samples which were provided by the University of Torontc, Electrical Engineering Department, experiments were conducted to identify the reflection properties at other observation angles than $1^{\circ}$. This experiment was performed using a gonioreflectometer.

\subsection{Reflection Properties Experiment}

Some small changes in the observation angle have been measured in other research, but only up to a maximum of $3^{\circ}$ has been considered. In general, little change in the reflection data has been recorded at these low values. For the calculation of target

luminance, the minimum limit must be much greater. If the areas of pavement in front of the target which contribute to the reflection of the light onto the target were measured in terms of the target size, the closest location would be at an observation angle of $45^{\circ}$. This requires that the change in reflection must be measured up to a minimum angle of $45^{\circ}$ as shown in Figure 14. For this research, an angle of $60^{\circ}$ was chosen as the upper limit of interest for the $\alpha$ parameter. Beyond $\alpha=60^{\circ}$, the calculation area in front of the target would be too small to be meaningful. 


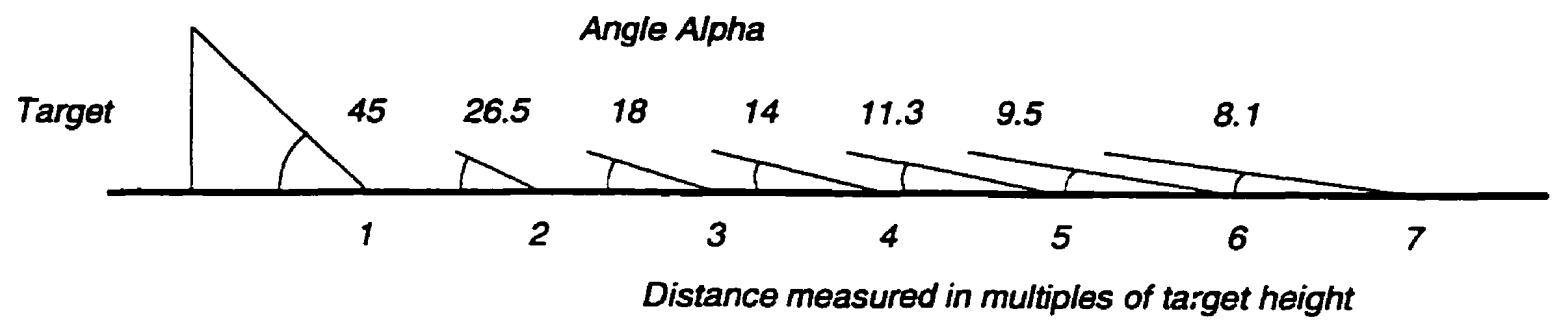

Figure 14 - Angular Limits of Reflection onto a Target Face

Similarly, the current methodology for measuring the reflection properties of pavement surfaces requires measuring from $\gamma=0^{\circ}$ to $85^{\circ}$ and $\beta$ from $0^{\circ}$ to $180^{\circ}$, as shown in Figure 15. The measurement intervals also change with the requirements of the pavement luminance calculation. This means that the reflection properties were measured more frequently at low values of $\tan (\gamma)$ than at high values representing luminaires which are close to vertically mounted over the calculation point. An example of a full r-table is shown in Table 6.

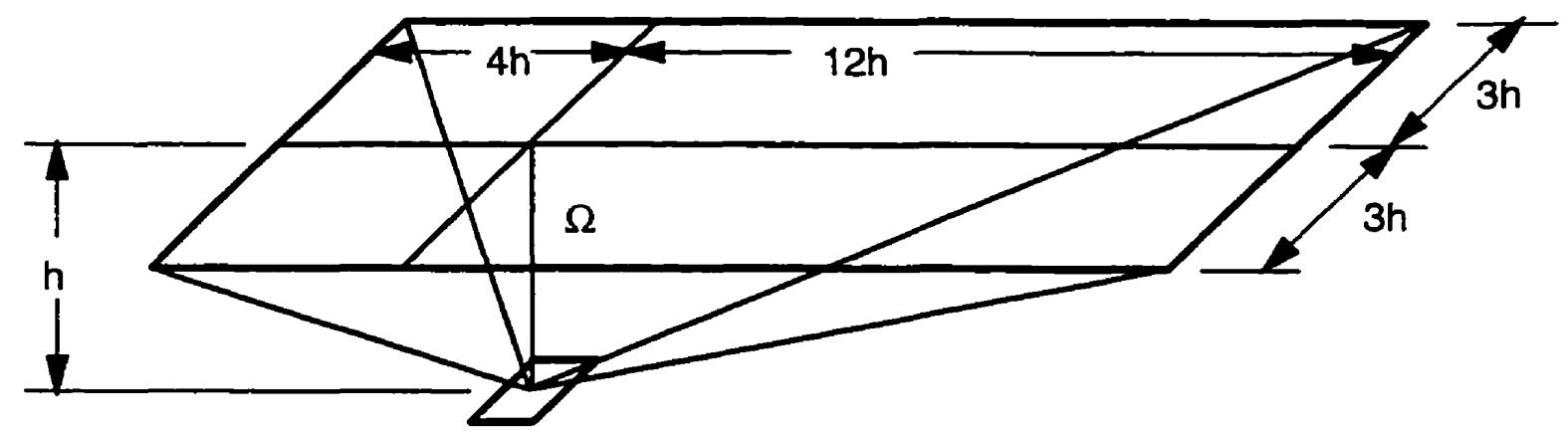

Figure 15 - Limits of Measurement for a Pavement Sample 
Table 6 - r-table for Sample Ontario L7-3

\begin{tabular}{|c|c|c|c|c|c|c|c|c|c|c|c|c|c|c|c|c|c|c|c|c|}
\hline $\begin{array}{l}\text { Tan } \\
(\gamma)\end{array}$ & $\beta$ & 2 & 5 & 10 & 15 & 20 & 25 & 30 & 35 & 40 & 45 & 60 & 75 & 90 & 105 & 120 & 135 & 150 & 165 & 180 \\
\hline 0 & 5724 & 5719 & 5765 & 55754 & 5672 & 5754 & 5800 & 5765 & 5707 & 5771 & 5800 & 5818 & 5847 & 5818 & 5800 & 5864 & 5859 & 5800 & 5724 & 5724 \\
\hline 0.25 & 5800 & 5812 & 5812 & 5771 & 5771 & 5771 & 5800 & 5765 & 5754 & 5719 & 5625 & 5614 & 5439 & 5398 & 5339 & 5298 & 5252 & 5205 & 5252 & 5258 \\
\hline 0.5 & 5509 & 5614 & 5427 & 5444 & 5380 & 5258 & 5164 & 5053 & 4931 & 4785 & 4680 & 4464 & 4353 & 4213 & 4184 & 4137 & 4184 & 4225 & 4225 & 4213 \\
\hline 0.75 & 5287 & 5304 & 5304 & 5118 & 4867 & 4552 & 4272 & 4120 & 3886 & 3700 & 3565 & 3297 & 3157 & 3105 & 3110 & 3151 & 3140 & 3192 & 3280 & 3250 \\
\hline 1 & 5333 & 5398 & 5345 & 54867 & 4184 & 3746 & 3326 & 3017 & 2830 & 2644 & 2544 & 2311 & 2223 & 2171 & 2223 & 2253 & 2305 & 2352 & 2393 & 2410 \\
\hline 1.25 & 5660 & 5812 & 5351 & 14400 & 3513 & 2912 & 2439 & 2223 & 2071 & 1926 & 1829 & 1660 & 1622 & 1600 & 1624 & 1683 & 1736 & 1792 & 1862 & 1816 \\
\hline 1.5 & 5940 & 5952 & 5698 & 33945 & 2784 & 2212 & 1862 & 1610 & 1526 & 1381 & 1347 & 1218 & 1185 & 1153 & 1216 & 1293 & 1324 & 1361 & 1426 & 1426 \\
\hline 1.75 & 6051 & 6080 & 4978 & 3233 & 2169 & 1614 & 1366 & 1204 & 1124 & 1027 & 975 & 904 & 845 & 904 & 918 & 964 & 994 & 1045 & 1109 & 1115 \\
\hline 2 & 6127 & 5987 & 4587 & 72533 & 1618 & 1244 & 1021 & 891 & 834 & 797 & 723 & 700 & 667 & 699 & 713 & 741 & 806 & 862 & 871 & 913 \\
\hline 2.5 & 5818 & 5287 & 3280 & 1552 & 960 & 732 & 525 & 550 & 497 & 490 & 471 & 466 & 423 & 443 & 457 & 490 & 532 & 572 & 597 & 610 \\
\hline 3 & 5333 & 4272 & 2270 & 961 & 624 & 490 & 413 & 364 & $38 ?$ & 348 & 317 & 301 & 298 & 308 & 317 & 348 & 383 & 423 & 446 & 451 \\
\hline 3.5 & 4791 & 3653 & 1538 & 3639 & 434 & 348 & 289 & 297 & 283 & 259 & 241 & 231 & 219 & 226 & 238 & 259 & 273 & 306 & 317 & 331 \\
\hline 4 & 4184 & 2719 & 1054 & 455 & 310 & 232 & 208 & 237 & 224 & 200 & 196 & 180 & 176 & 192 & 196 & 209 & 236 & 242 & 261 & 273 \\
\hline 4.5 & 3746 & 2146 & 747 & 7353 & 233 & 200 & 178 & 168 & 178 & 164 & 157 & 133 & 142 & 148 & 158 & 175 & 188 & 208 & 210 & $2[4$ \\
\hline 5 & 3373 & 1730 & 597 & $7 \quad 247$ & 195 & 175 & 158 & 133 & 132 & 146 & 129 & 112 & 116 & 131 & 140 & 143 & 154 & 172 & 187 & 202 \\
\hline 5.5 & 2906 & 1325 & 441 & 235 & 170 & 128 & 119 & 113 & 101 & 102 & & & & & & & & & & \\
\hline 6 & 2626 & 1053 & 364 & 189 & 139 & 112 & 111 & 100 & 92.9 & & & & & & & & & & & \\
\hline 6.5 & 2311 & 845 & 294 & 4157 & 115 & 98.5 & 95.1 & 87.3 & & & & & & & & & & & & \\
\hline 7 & 2033 & 661 & 247 & 7136 & 104 & 87.8 & 82.2 & 79.7 & & & & & & & & & & & & \\
\hline 7.5 & 1829 & 586 & 233 & $3 \quad 121$ & 95.5 & 83.6 & 77.5 & & & & & & & & & & & & & \\
\hline 8 & 1664 & 493 & 200 & 111 & 81.1 & 71.4 & 71 & & & & & & & & & & & & & \\
\hline 8.5 & 1557 & 427 & 174 & 96.2 & 79.8 & 68.6 & 68 & & & & & & & & & & & & & \\
\hline 9 & 1321 & 364 & 160 & 92.4 & 76.6 & 64.9 & & & & & & & & & & & & & & \\
\hline 9.5 & 1260 & 331 & 141 & 90.9 & 68.6 & 63.5 & & & & & & & & & & & & & & \\
\hline 10 & 1171 & 273 & 138 & 79.7 & 61.2 & 62.6 & & & & & & & & & & & & & & \\
\hline 10.5 & 1157 & 261 & 122 & 72.8 & 62.6 & 55.6 & & & & & & & & & & & & & & \\
\hline 11 & 1017 & 236 & 113 & 375.9 & 59.2 & 54.6 & & & & & & & & & & & & & & \\
\hline 11.5 & 919 & 240 & 106 & 564.9 & 55 & & & & & & & & & & & & & & & \\
\hline 12 & 820 & 210 & 99.7 & $\begin{array}{ll}7 & 65.2\end{array}$ & 54.1 & & & & & & & & & & & & & & & \\
\hline
\end{tabular}

For this experiment, the entire BRDF was measured on the pavement samples using the angular limits specified above. The measurement intervals used for this experiment were chosen to match the existing r-table and the requirements of the target data as closely as possible. The chosen values are:

$\alpha=1^{\circ}, 2^{\circ}, 3^{\circ}, 5^{\circ}, 7^{\circ}, 10^{\circ}, 12^{\circ}, 15^{\circ}, 20^{\circ}, 30^{\circ}, 45^{\circ}$ and $60^{\circ}$

$\beta=0^{\circ}, 5^{\circ}, 10^{\circ}, 15^{\circ}, 20^{\circ}, 25^{\circ}, 30^{\circ}, 35^{\circ}, 45^{\circ}, 60^{\circ}, 70^{\circ}, 90^{\circ}, 105^{\circ}, 120^{\circ}, 135^{\circ}, 150^{\circ}$ and $180^{\circ}$ 
$\gamma=0^{\circ}, 15^{\circ}, 30^{\circ}, 45^{\circ}, 60^{\circ}, 70^{\circ}, 75^{\circ}, 80^{\circ}$, and $85^{\circ}$

The $\delta$ angle of the BRDF was also investigated. This angle, although not used in the pavement luminance calculation, is used for the target luminance calculation. The change in reflection due to this angle was measured by rotating the sample under a fixed angle of incidence and observation. The reflection was measured at every $15^{\circ}$ of $\delta$ for one complete rotation of the sample.

\subsection{Equipment}

\subsubsection{Samples}

Approximately one hundred samples of pavement surfaces were obtained from the University of Toronto, Electrical Engineering Department for this investigation. The samples were used during the early 1980's for developing the Canadian atlas of pavement reflection (Dmitrevsky and Bassett [1983]). Most of the previous North American work cited on pavement materials is based on the results of these investigations.

The original samples were obtained from each province in Canada, and some selected locations in the United States and Europe. Generally, the samples are $18 \mathrm{~cm}$ diameter disks which have been extracted by a normal cylindrical coring machine. The samples have been previously measured using a $1^{\circ}$ observation angle gonioreflectometer system. The samples represent a complete cross section of all of the road classes giving $\mathrm{S} 1$ values of 0.2 to 2.4 and S2 values of 1.3 to 3.7. Each sample is marked for the direction travel. The standard reflection data $\left(\alpha=1^{\circ}\right)$ for each sample was provided as well as, 
when available, their material composition, age, strength and type of traffic usage.

For our investigation, twenty samples were chosen based on their $\mathrm{S} 1$ values in order to provide a cross section of all R-Classes. The characteristics of the chosen samples are shown in Table 7.

Table 7 - Selected Samples Used for the Reflection Experiment

\begin{tabular}{||l|l|l|l|l|l||}
\hline Sample Name & R & S1 & S2 & $\mathbf{Q}_{0}$ & Pavement Type \\
\hline Alberta \#1 & R1 & 0.20 & 1.41 & 0.074 & Asphalt Seal Coat \\
\hline British Columbia N4 Hwy19 & R3 & 1.09 & 2.45 & 0.077 & Asphalt Top Lift \\
\hline Manitoba PR 200 1BWP & R2 & 0.67 & 1.86 & 0.127 & Concrete \\
\hline New Brunswick Rt2 \#5 & R1 & 0.44 & 1.81 & 0.072 & Asphalt Top Seal \\
\hline Nova Scotia \#11 & R1 & 0.36 & 1.45 & 0.051 & Asphalt Seal Coat \\
\hline Nova Scotia \#15 & R3 & 0.87 & 2.18 & 0.073 & Asphalt \\
\hline Ontario 19-14 & R3 & 0.97 & 2.26 & 0.067 & Asphalt Dense Friction Course \\
\hline Ontario 19-17 & R4 & 1.39 & 2.78 & 0.079 & Asphalt Dense Friction Course \\
\hline Ontario 19-3 & R4 & 2.04 & 3.25 & 0.097 & Asphalt Dense Friction Course \\
\hline Ontario 19-7 & R4 & 2.17 & 3.34 & 0.092 & Asphalt Dense Friction Course \\
\hline Ontario L7-2 & R2 & 0.84 & 2.00 & 0.107 & Asphalt Dense Friction Course \\
\hline Ontario L7-3 & R3 & 1.05 & 2.13 & 0.123 & Asphalt Dense Friction Course \\
\hline Ontario L7-4 & R3 & 0.88 & 2.01 & 0.106 & Asphalt Dense Friction Course \\
\hline PEI \#2-K & R2 & 0.62 & 1.95 & 0.094 & Asphalt Surface Coat \\
\hline Quebec \#4 & R2 & 0.63 & 1.98 & 0.122 & Concrete \\
\hline Quebec \#6 & R2 & 0.48 & 1.75 & 0.10 & Concrete \\
\hline Saskatchewan WL \#10 & R3 & 1.31 & 2.50 & 0.104 & Asphalt Hot Mix \\
\hline Saskatchewan WL \#32 & R2 & 0.68 & 1.88 & 0.069 & Asphalt Hot Mix \\
\hline Saskatchewan WL \#37 & R2 & 0.85 & 2.01 & 0.076 & Asphalt Hot Mix \\
\hline Saskatchewan WL\#8 & R3 & 1.07 & 2.28 & 0.092 & Asphalt Hot Mix \\
\hline
\end{tabular}


At the time of this investigation, the samples were at least twelve years old and had not been actively used for several years. The samples were maintained in crates and in plastic bags to protect them from dust, but they were not kept in a temperature or humidity controlled room and some of them were not able to be salvaged.

The reflection experiment was performed with dry pavement samples only. When a pavement is wet, the reflection becomes much more specuiar. Testing wet pavement is extremely difficult. Humidity control and a definition "wet" are both issues with this type of testing. Due to the complexity already introduced with the change in observation angle, it was decide to work only with dry surfaces.

\subsubsection{Gonioreflectometer}

To obtain the data for the variable observation angle reflection properties, a gonioreflectometer was developed and automated. A schematic of the machine is shown in Figure 16 and a photograph is shown in Appendix C. This instrument uses a movable arm which allows for the change in the $\gamma$ and $\beta$ angles of the light source and another arm which allows for the change in $\alpha$ angle of a luminance meter. The gonioreflectometer allows for the adjustment of $\beta$ from $0^{\circ}$ to $180^{\circ}$, of $\gamma$ from $0^{\circ}$ to $+85^{\circ}$ and $\alpha$ from $1^{\circ}$ to $85^{\circ}$ with $0.5^{\circ}$ accuracy for all axes. Because there is a separate arm for the luminance meter, the light source came into contact with the luminance meter when the measurement geometry was such that $\alpha=(90-\gamma)$ and $\beta=180^{\circ}$. When this occurred, the measurement was taken at $\beta=170^{\circ}$ and recorded as such. The apparatus uses a 
diffuse Quartz Halogen light source and a portable Minolta luminance meter with a 20॰ measuring field. The luminance readings were recorded by a personal computer which also controlled some of the movement of the axes. The output of this instrument is a measurement of the pavement luminance. This result can then be translated into a reflection factor, q, or a reduced reflection factor, $r$.

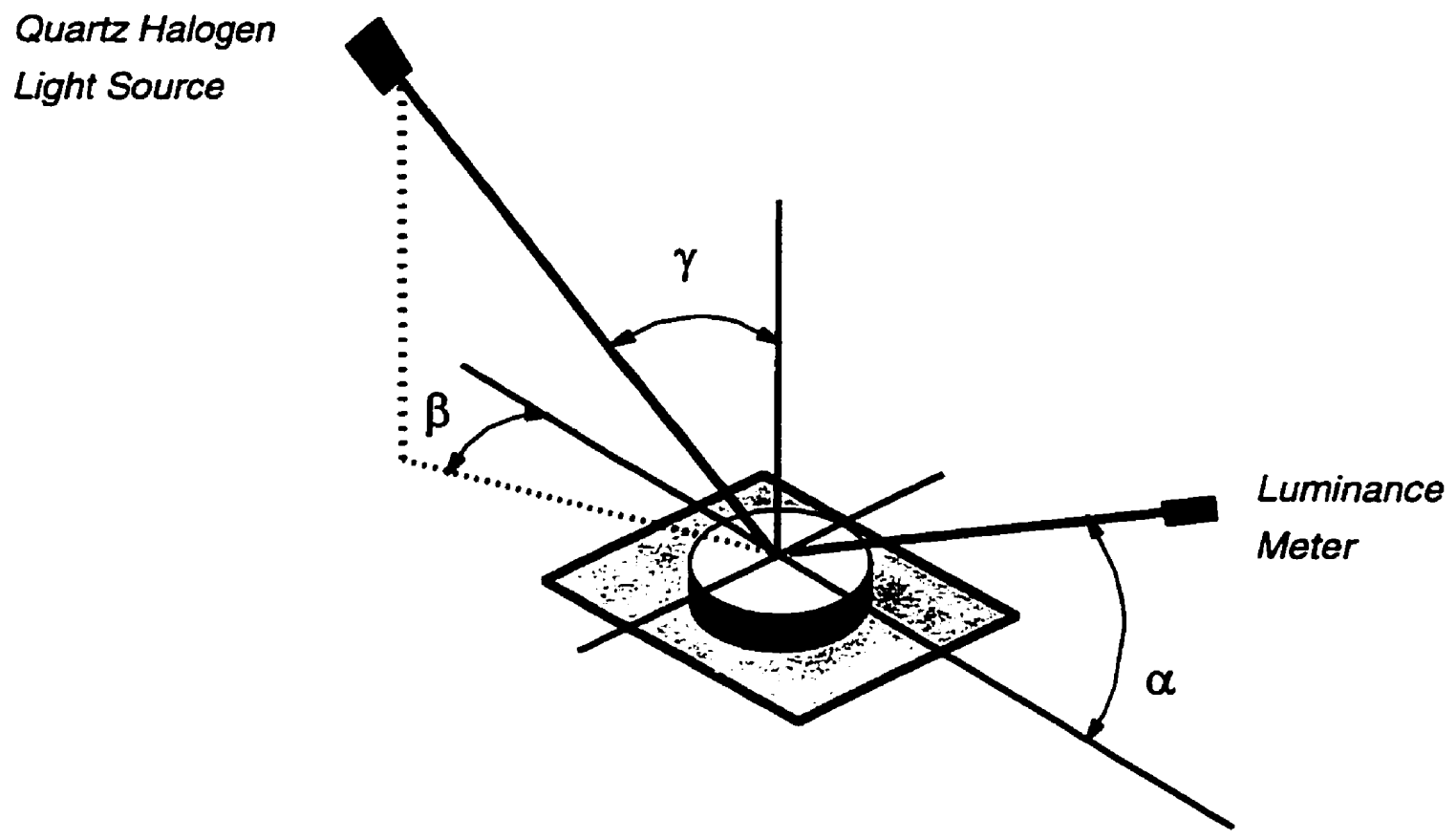

Figure 16 - Schematic of the Gonioreflectometer

This instrument is different from other equipment used for pavement sample reflection measurements. The University of Toronto and many European systems use a turntable with the detector attached. The light source is attached to an overhead rail and moved horizontally to change the $\tan (\gamma)$ angle. The sample turntable is then rotated to change the $\beta$ angle. This measurement methodology allows the reduced reflection factor, $r$, to be 
measured directly. This methodology was not practical for our measurements. The space requirements and the need to change the $\alpha$ angle required the construction of a true circular gonioreflectometer.

The acceptance aperature of the system was defined by the design on the Minolta luminance meter. The $20^{\circ}$ aperature was used as a design constraint of the system. The length of the arms of the gonioreflectometer were then chosen for a minimum $18 \mathrm{~cm}$ sample. At the lowest $\alpha$ angle of interest, $\alpha=1^{\circ}$, the size of the longest dimension of the luminance meter's acceptance field is $18 \mathrm{~cm}$. This means that a sample of less than 18 $\mathrm{cm}$ cannot be measured on this system.

The sample orientation for the measurements in this research was not the typical orientation for pavement reflection measurements. Usually, the angle of interest is from the driver's viewpoint. This means that the sample is oriented with the luminance meter in line with the direction of travel and at the oncoming traffic side of the sample. For this research, the reflection interest was not towards the driver, but away from the driver towards a target. This means that the sample was located in the reverse direction to the typical orientation. The driving observer then returns to the calculation through the target luminance calculation.

The 50 Watt quartz halogen light source was powered using a variable stabilized DC power supply. An illuminance meter was placed over the pavement sample at the 
beginning of a test and the intensity of the light source onto the sample was calculated. The intensity was then checked using the illuminance meter whenever the $\alpha$ angle was adjusted, usually every 100 readings. If the intensity had changed, the power supply was adjusted back to the original value. Stability checks were made on the light source by measuring the luminance from a sample every 15 seconds for two hours without adjusting the system in any way. These results are shown in Figure 17. The light source was also warmed up 30 minutes to 1 hour before measurements were made to allow the lamp output to stabilize. After stabilization, the lamp showed a $1.03 \%$ average deviation in the output at a constant voltage. 


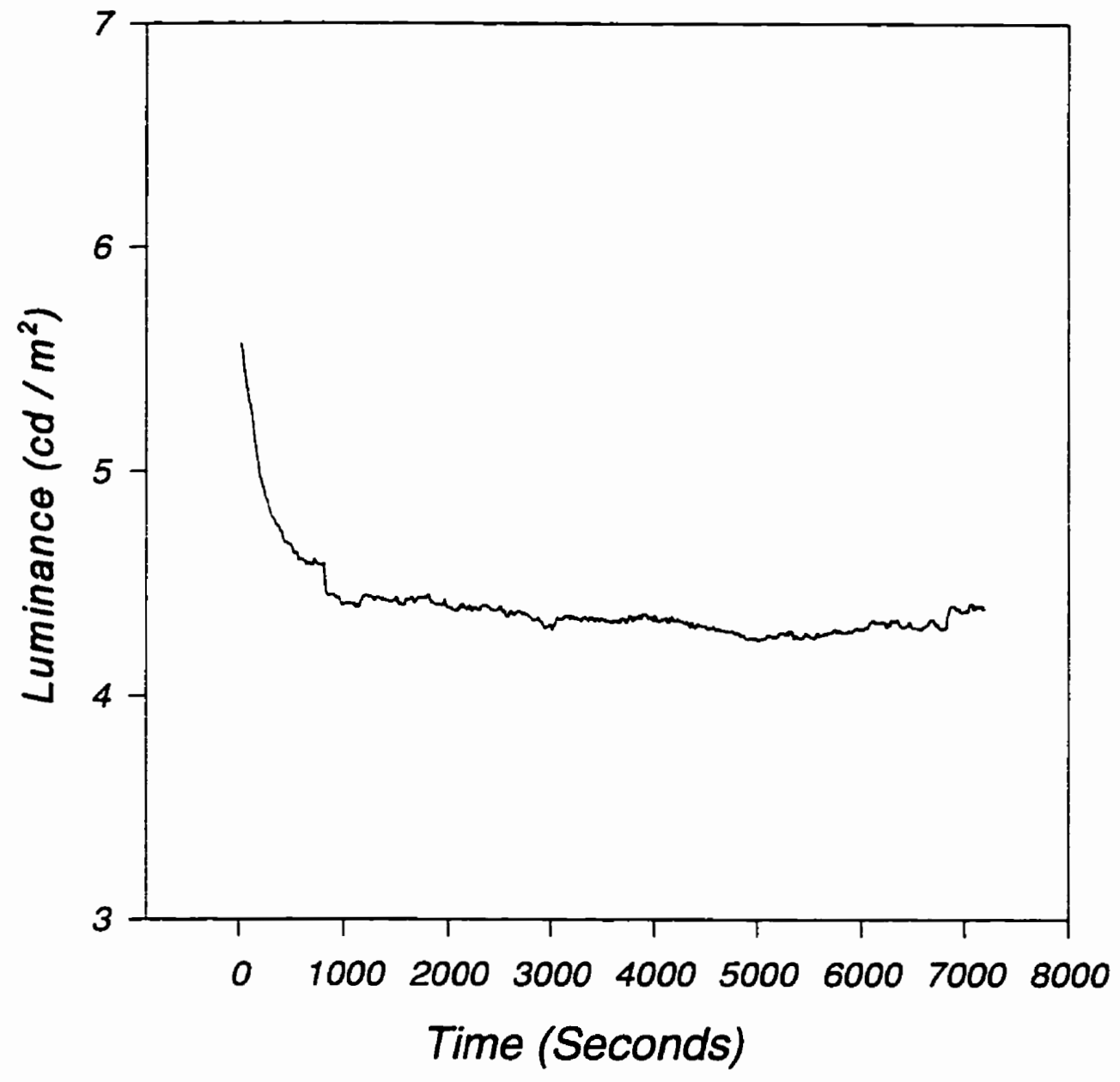

Figure 17 - Lamp Stabilization Curve

For control of stray light from the light source into the luminance meter, the experiments took place in a darkened room with black light-absorbing curtains and a baffle tube was designed for the front of the luminance meter. The tube contained two entrance pupils which would limit the stray light entering the system. Similarly, since the luminance meter arm was relatively short, approximately $80 \mathrm{~cm}$, the luminance meter was at the 
extent of its focussing capabilities. To allow the meter to focus more clearly, a close-up lens was used for the data collection.

Early results from this equipment were reported in Gibbons and Adrian [1994]. These data showed a large jump in the $\alpha=3^{\circ}$ data. This appeared to be an anomaly in the results and led to a redesign of the glare tube and a change in the luminance meter acceptance angle. The new system results seem to have dealt with the problems associated with the equipment.

The calibration of the machine took place in two formats. Since a tube was used for glare control, the entrance pupil of the meter changed, requiring a multiplying factor between the bare lens reading and the reading with the tube and lens. This calculation was performed for several variations of $\alpha$ and $\gamma$. The result was the use of a 1.223 scaling factor on all data read. The second calibration issue was the geometrical calibration. This required measuring the reflectance of the light source from a reflectance standard and comparing the results to the calculated expected results. This was performed using a Magnesium Oxide reflectance standard and measuring it at varying $\alpha$ and $\gamma$ angles. The results of this calibration are shown in Figure 18. In this comparison, the angle of interest is the angle between the detector and the light source. This is plotted across the $\mathrm{x}$-axis of the figure. The two data curves were found first by varying $\alpha$ with $\gamma=0^{\circ}$ and next by varying $\gamma$ with $\alpha=90^{\circ}$. The angle between the detector and source is equal to $90-\alpha$ in the $\alpha$ varying case and equal to $\gamma$ in the $\gamma$ varying case. 
The curve labeled as calculated is determined using an equation for the change in the reflectance of a Magnesium Oxide sample with the change in observation angle. This equation is presented in CIE Report 46 [1979]. The equation is shown in Equation 37.

$$
\text { Reflectivity }=1-1.3 \cdot \sin \left(\frac{\alpha}{2}\right)^{4}
$$

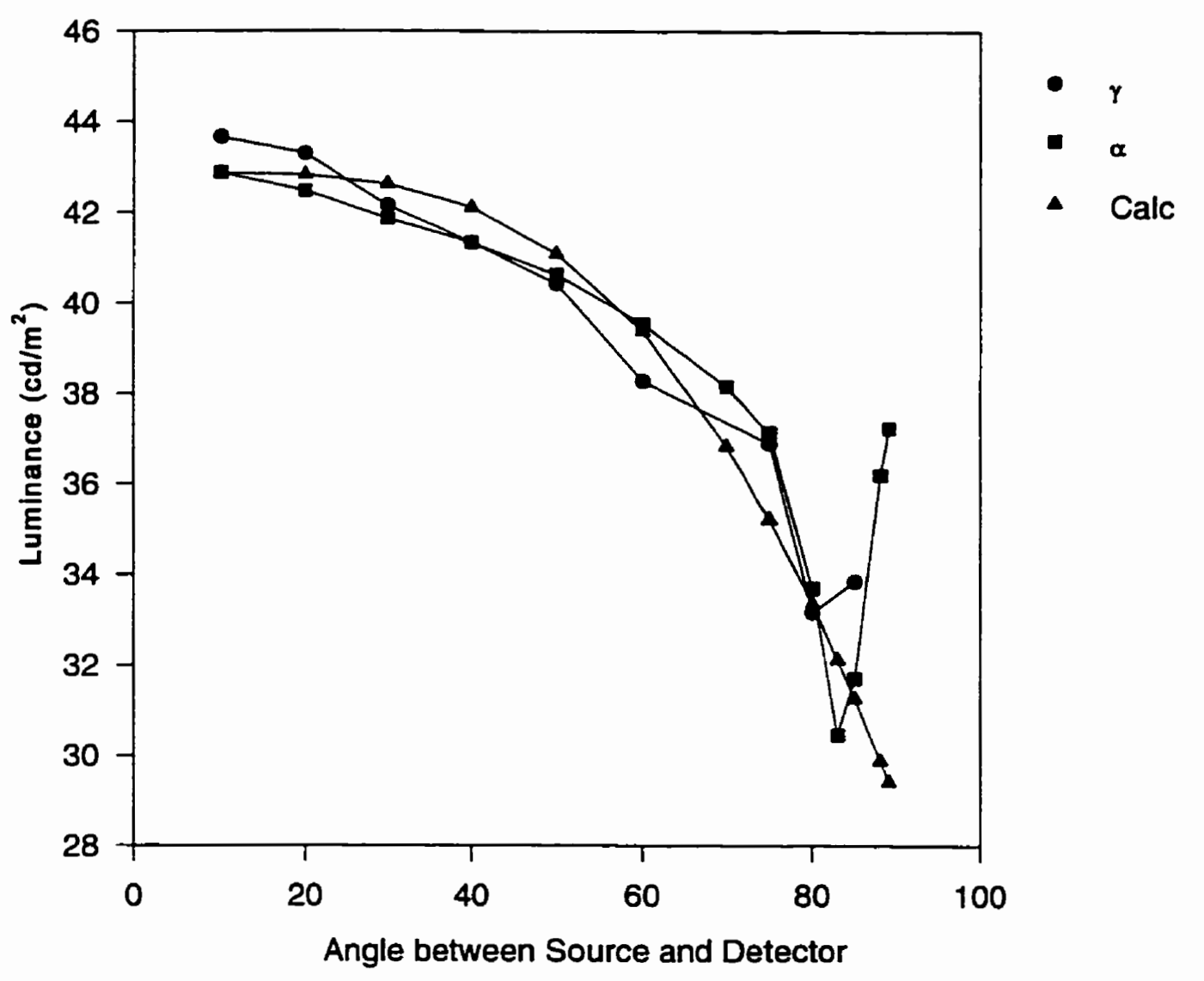

Figure 18 - Calibration of Gonioreflectometer with Magnesium Sulphate Reflectance Standard

Where $\alpha$ is the observation angle.The reflectance standard is two inches in diameter and 
smaller than the acceptance angle of the luminance meter for small values of $\alpha$. This resulted in the high inaccuracy in the calibration curve at the small $\alpha$ angles. This variability would not be evident with the larger pavement sample.

As a final check on the results, a cross check between the measurements at $\alpha=1^{\circ}$ was compared to those of the University of Toronto. The comparison shows a good correlation of measured $\mathrm{q}_{0}, \mathrm{~S} 1$ and $\mathrm{S} 2$ values to those measured by the University of Toronto. These results are shown in Chapter 5.

\subsection{Uncertainty}

The final number of reflection data points for each sample was 1872 . In order to estimate the error associated with each data point, rather than test each sample extensively, it was decided that a general uncertainty would be developed for each measurement location and then used as the data point uncertainty. This uncertainty was developed by testing a single sample several times. The experimental standard deviation of the mean was used as the measurement uncertainty. As the complexity of the experiment increased, and sample types and sample roughnesses were changed, the uncertainty also had to be verified if it were to be valid for these data types. The uncertainty calculation was performed based on the ISO/TAG 4/WG 3: June 1992 document "Guide to the Expression of Uncertainty in Measurement".

The sample Saskatchewan \#10 was chosen for the uncertainty measurement. It is an R3 
pavement surface with $S 1$ and $S 2$ values of 1.31 and 2.50 respectively.

For this uncertainty calculation, the entire BRDF of the sample Saskatchewan \#10 was measured five times. For each run, the sample was removed from the gonioreflectometer and then remounted as if in a brand new experiment. The percent uncertainty, the sample standard deviation of the mean, was then calculated for each $\alpha, \beta$, and $\gamma$ angle combination. The sample standard deviation of the mean of the measurements is shown in Equation 38.

$$
\mu_{q}=s^{2}(\bar{q})=\frac{s^{2}\left(q_{k}\right)}{n}
$$

where $\mu_{q}$ is the uncertainty of the mean of $q_{k}$, the repeated measurement. For the actual data collection, the data read from the system is considered to be the mean and the uncertainty is applied to this value.

The results for each of the angular parameters are shown in Figures 19 to 21 . The average error for the entire experiment is $2.80 \%$. It is noteworthy that there appears to be no trend in the error data. It was expected that the errors would be greatest at the extremes of the measurement limits, however the resulting errors are spread evenly over the measurement range. This estimated error also differs from that published in Gibbons and Adrian [1996]. The error was re-evaluated and recalculated based on an improved method for mounting the sample on the gonioreflectometer. 


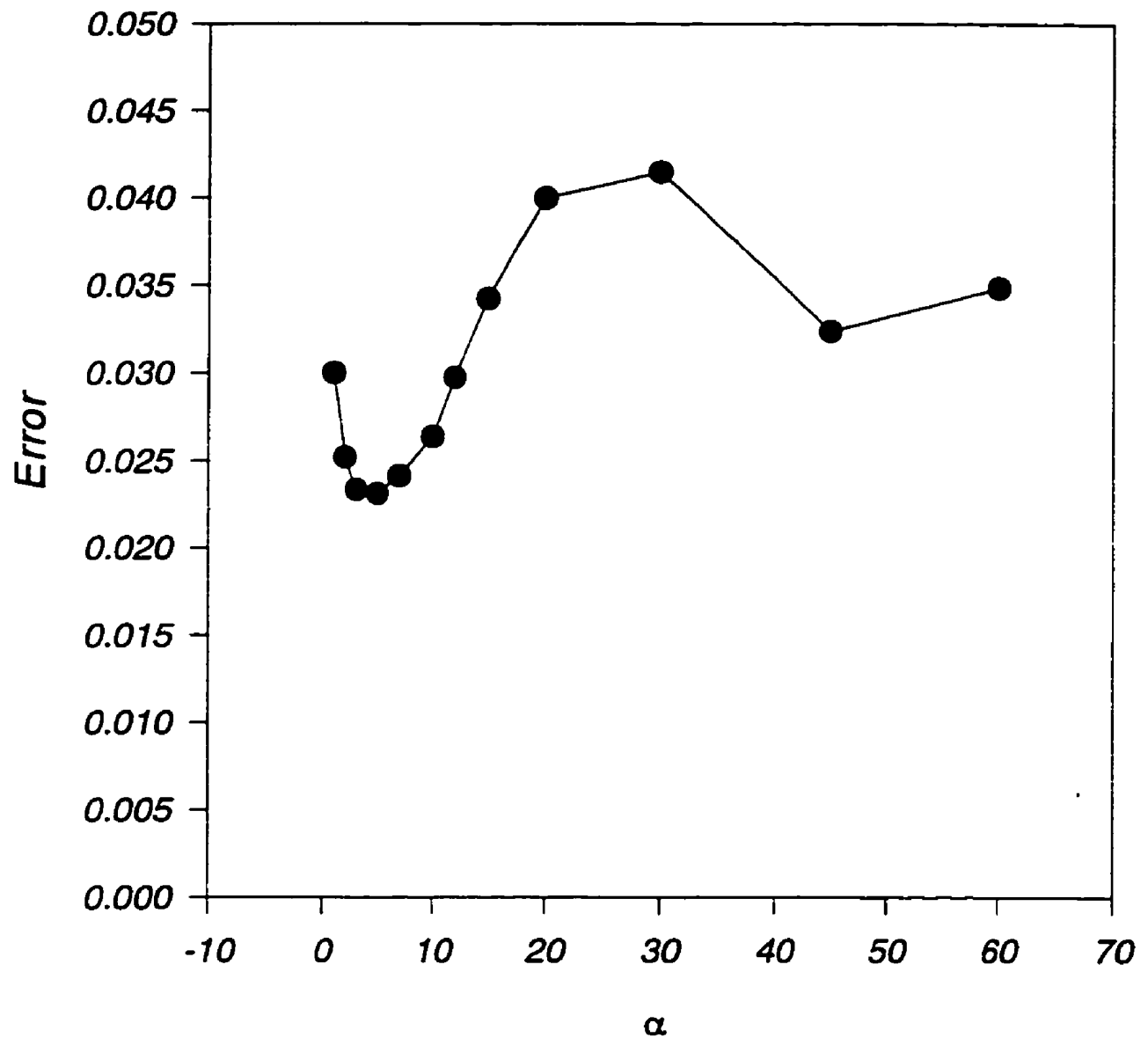

Figure 19 - Average Normalized Uncertainty in $\alpha$ 


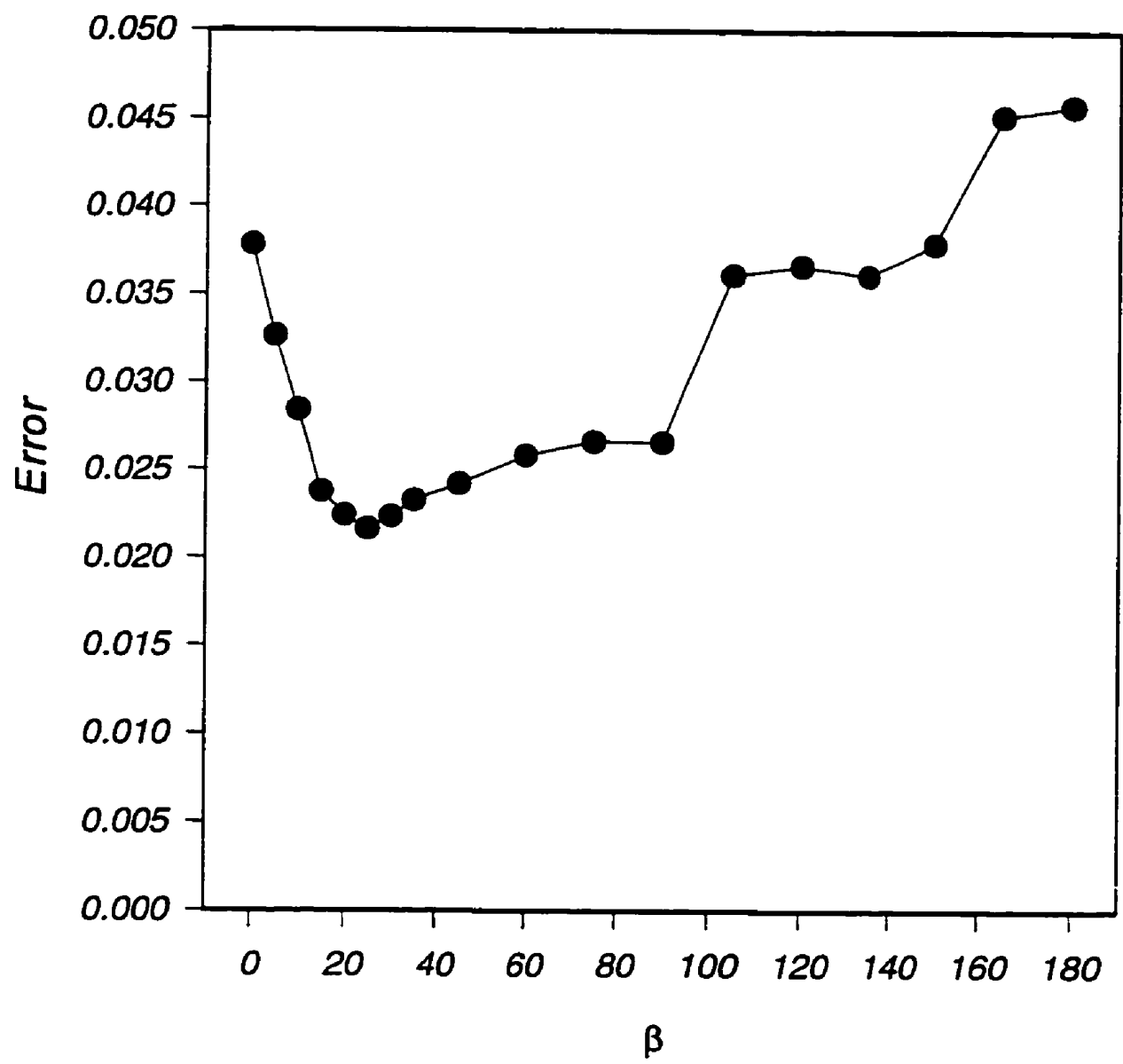

Figure 20 - Average Normalized Uncertainty in $\beta$ 


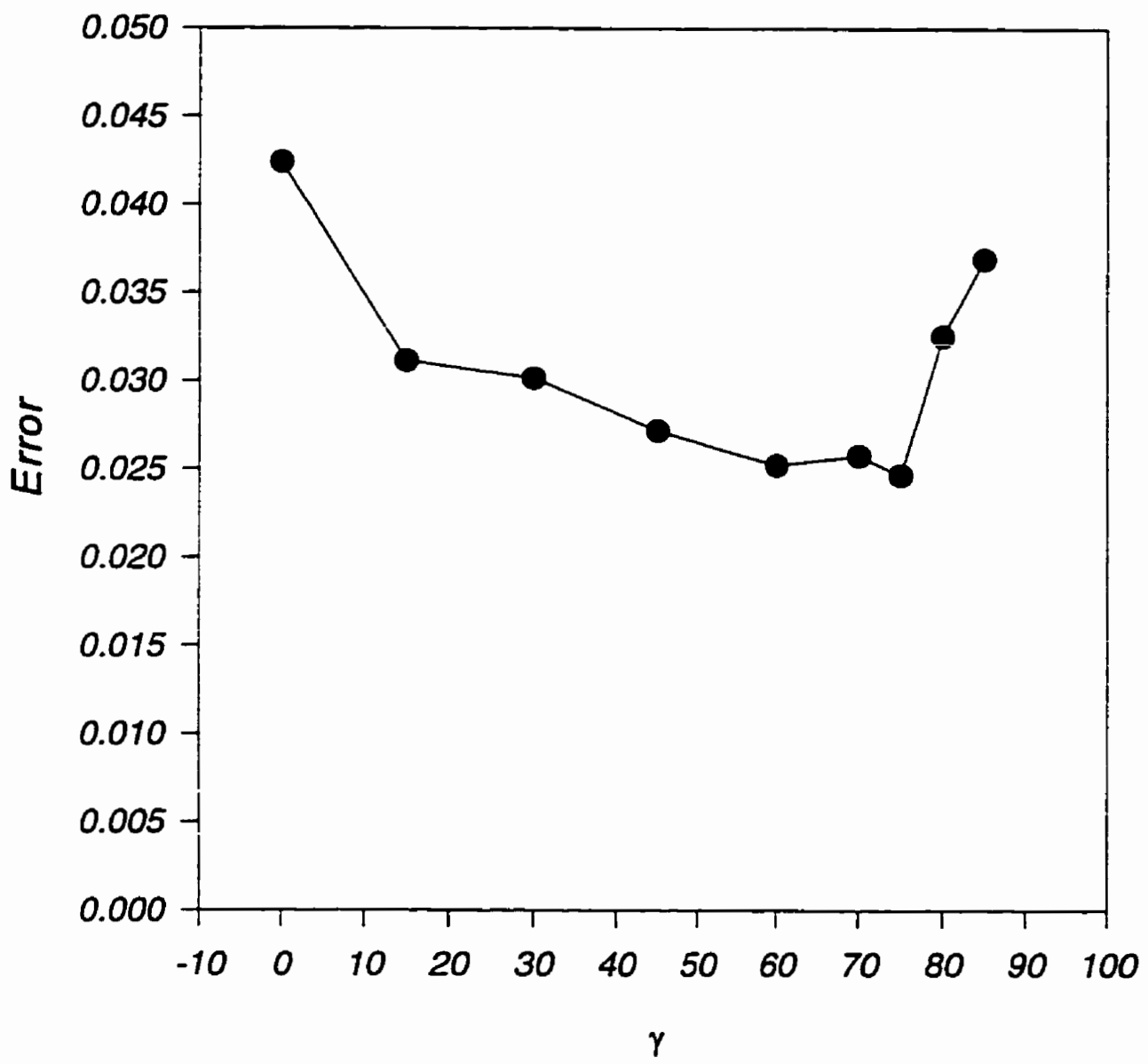

Figure 21 - Average Normalized Uncertainty in $\gamma$

The uncertainty for the factors of $q_{0}, S 1$ and $S 2$ were calculated based on the repeated measures method. These uncertainties are shown in Table 8. 
Table 8 - Uncertainty for $q_{0}, S 1, S 2$

\begin{tabular}{|c|c|}
\hline Criteria & $\begin{array}{c}\text { Percent } \\
\text { Uncertainty }\end{array}$ \\
\hline $\mathrm{q}_{0}$ & 3.25 \\
\hline $\mathrm{S} 1$ & 3.43 \\
\hline $\mathrm{S} 2$ & 5.6 \\
\hline
\end{tabular}

The other aspects which were investigated were the effect of material type and roughness on the magnitude of the uncertainty. For each of these comparisons, the full sample reflection characteristics were not measured. The following angles were selected:

$$
\begin{aligned}
& \alpha=1^{\circ}, 5^{\circ}, 15^{\circ}, 45^{\circ} \\
& \beta=10^{\circ}, 30^{\circ}, 75^{\circ} \\
& \gamma=15^{\circ}, 30^{\circ}, 75^{\circ}
\end{aligned}
$$

To investigate the change in the uncertainty due to material type, sample Quebec \#4 which is a concrete sample of class R2 with S1 and S2 values of 0.63 and 1.98 respectively was tested at the angles specified. The results compared to the uncertainty from the Saskatchewan \#10 testing are shown in Figure 22. This figure shows the percent uncertainty average across the angular measurements of $\alpha, \beta$, and $\gamma$. It can be seen that the average uncertainty in all parameters is less with the concrete sample than with the asphalt sample. An Analysis of Variance (ANOVA) was performed with this data and is shown in Table 9. There is a slight significance in the material type but the mean of the results for the concrete sample is less than that of the asphalt, so the asphalt uncertainty represents a worst case in the material type comparison. 

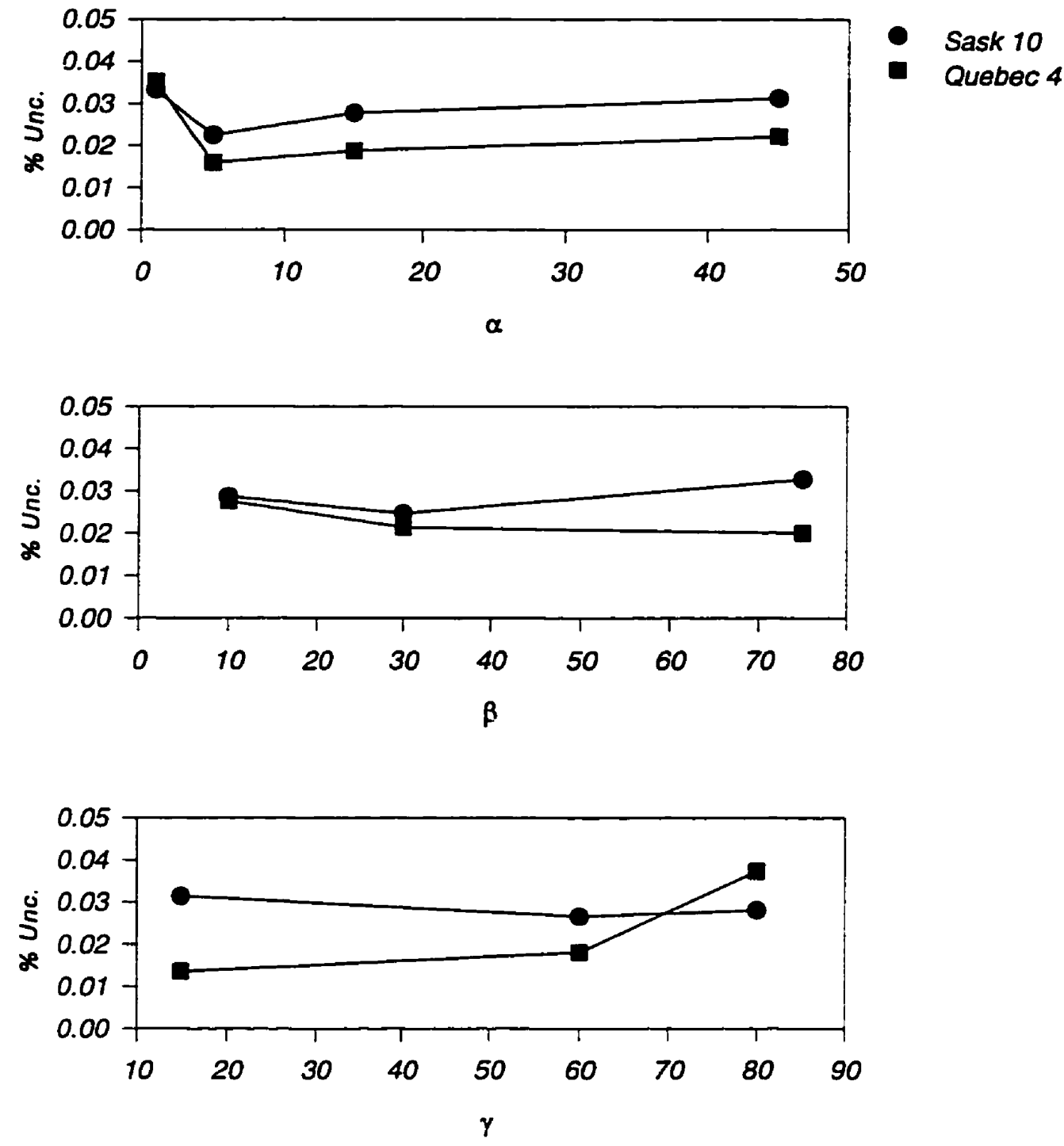

Figure 22 - Percent Uncertainty Comparison for Different Materials Types 
Table 9 - ANOVA for Pavement Material Type

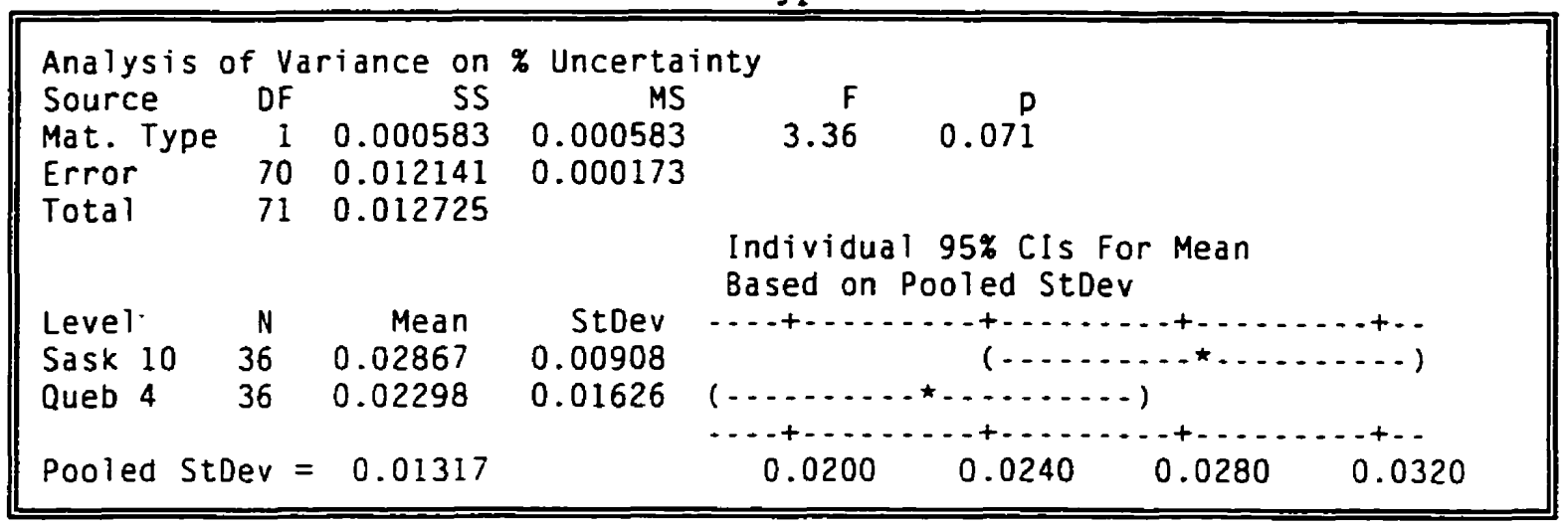

Similarly, to test the impact of surface roughness, Nova Scotia 15 , which is a relatively smooth sample, was tested and compared to Saskatchewan \#10 and shown in Figure 23. As in Figure 22, the average uncertainty for each of the angular parameters is compared. In this comparison, there is apparently no dependence of the uncertainty on the sample type. An ANOVA of the means was performed on this data. No significance was found in the sample type with a percent confidence of $46 \%$. The ANOVA table is shown in Table 10. 

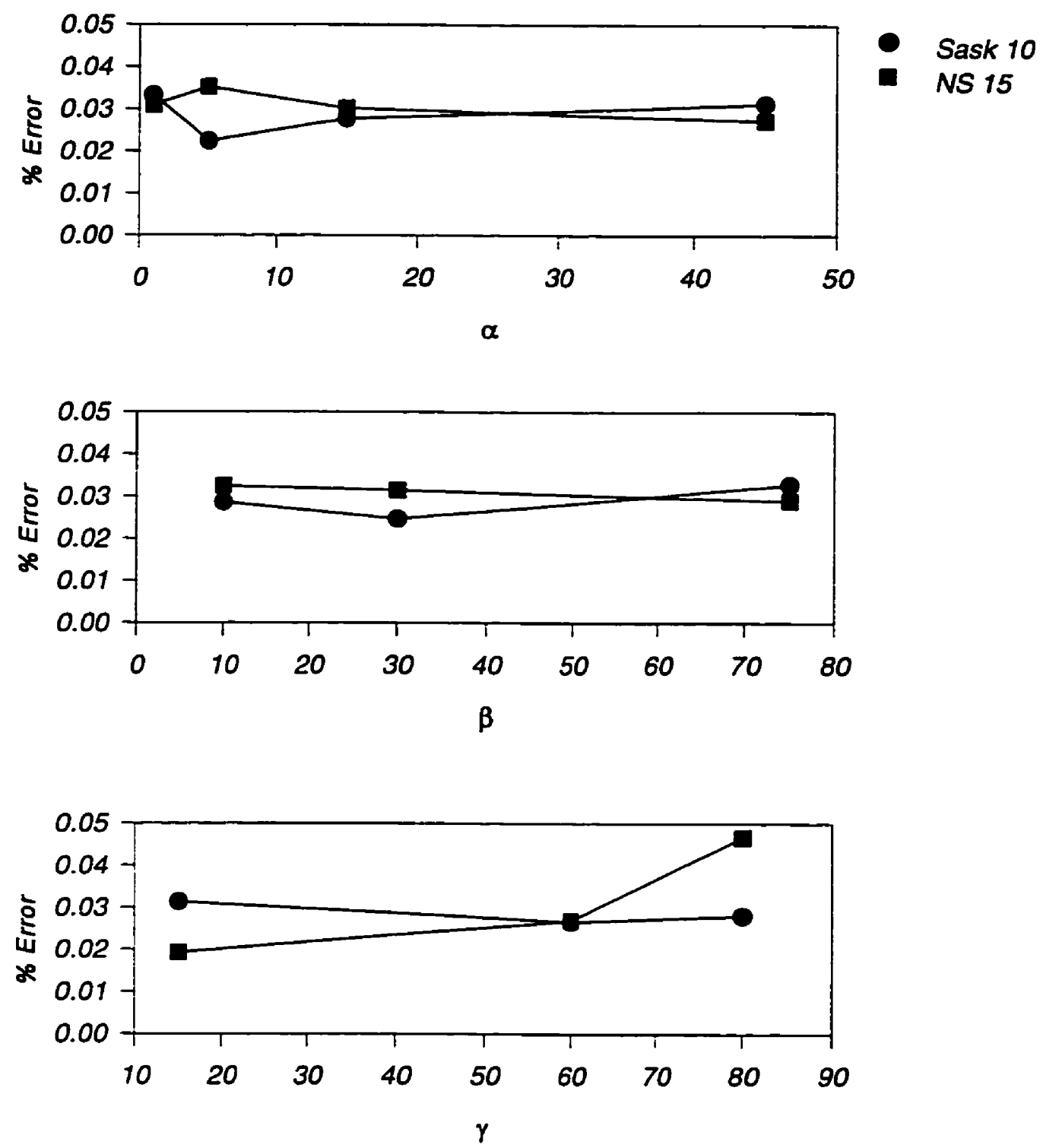

Figure 23 - Percent Uncertainty Comparison for Different Roughness Samples 
Table 10 - ANOVA Table for Sample Roughness

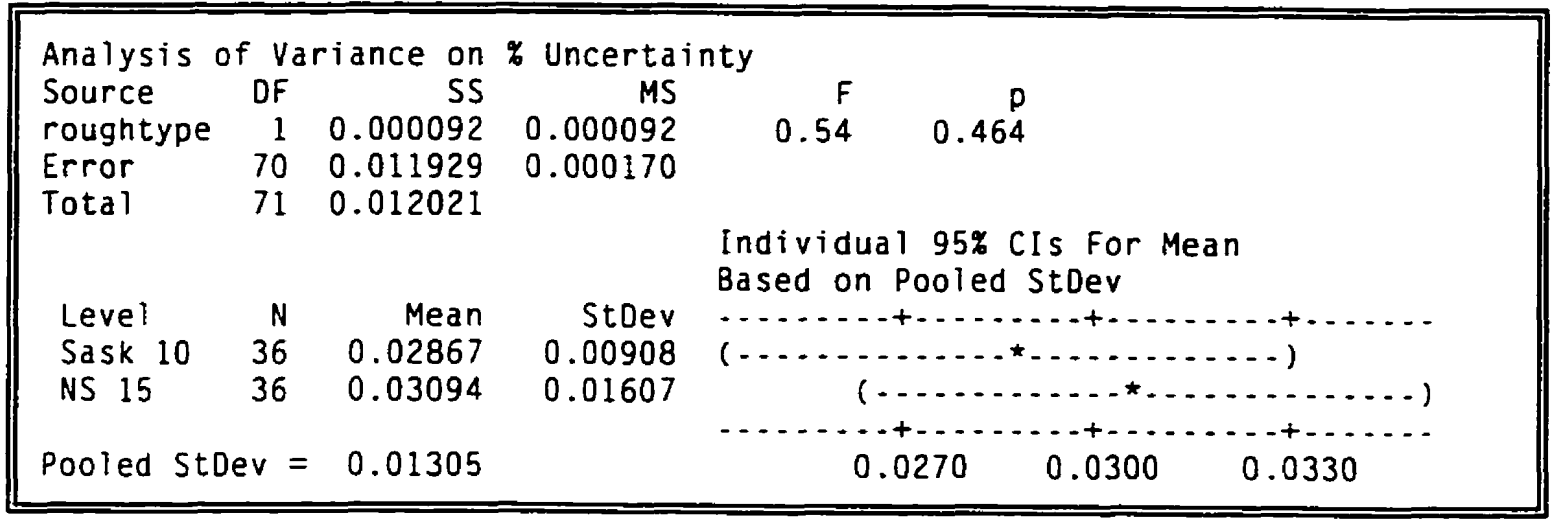

The final issue which influenced the measurements of uncertainty was a random versus non-random measurement procedure. Due to the design of the equipment, the standard measurement was based on measuring all $\beta$ angles at a given $\alpha$ and $\gamma$, adjusting $\gamma$ and then finally adjusting $\alpha$ after all $\beta$ and $\gamma$ combinations were complete. A set of measurements was made in which all measurements were random across $\gamma$ and $\beta$. The sample used for these experiments was Sasketchewan \#10. The uncertainty for these measurements was then calculated and compared to the non-random experiment in Figure 24. Here again, the uncertainty is analyzed in an ANOVA. The results are shown in Table 11. This analysis also shows a slight dependence on the measurement method. Like the material type dependence, the random experiment shows a lower uncertainty than the sequential methodology. This difference is likely due to errors which develop in positioning the sample for each test and in positioning the luminaire for each measurement. 

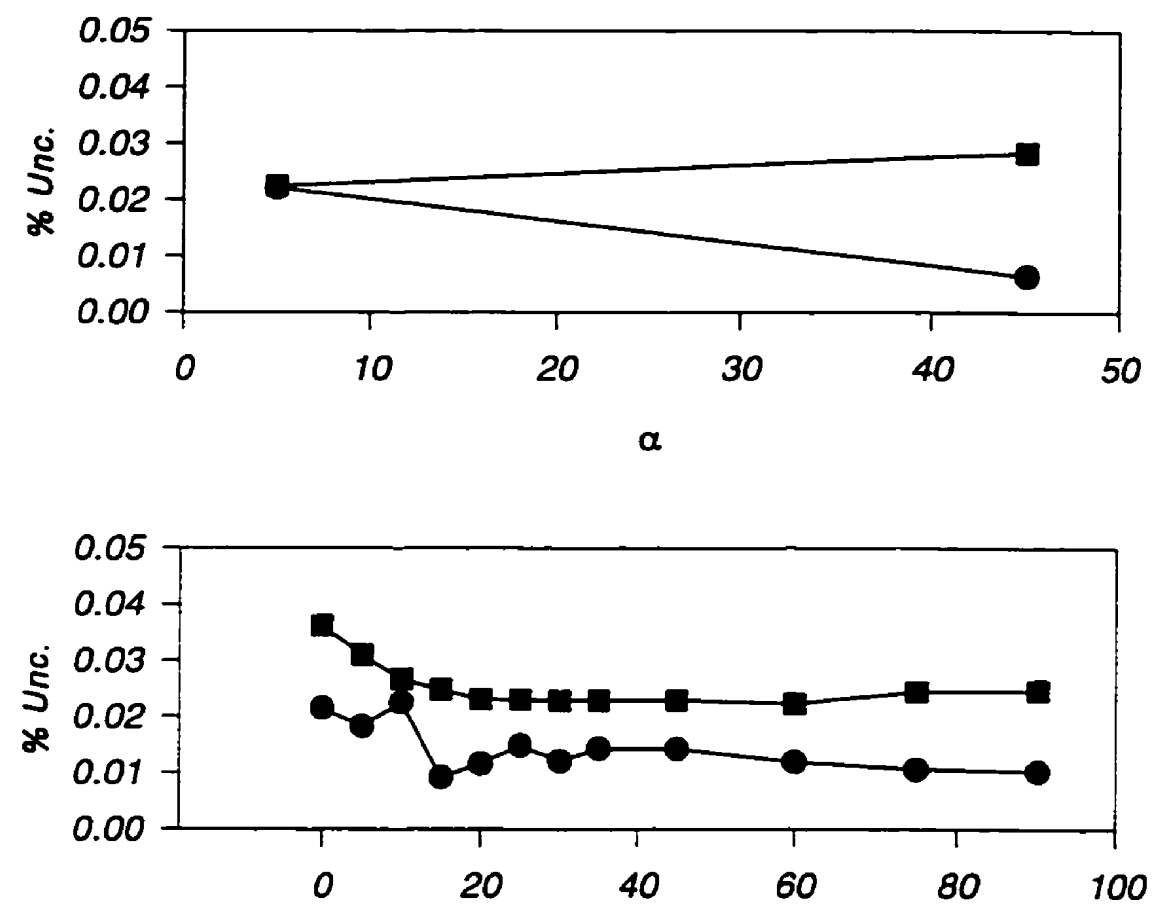

$\beta$

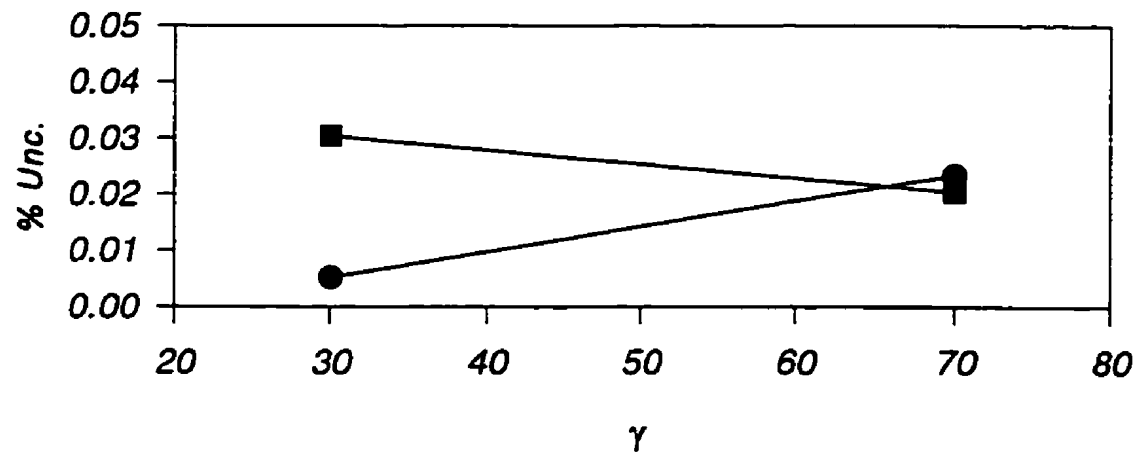

Figure 24 - Per̃cent Uncertainty Comparison for Data Collection Methodologies 
Table 11 - ANOVA Table for Data Collection Method

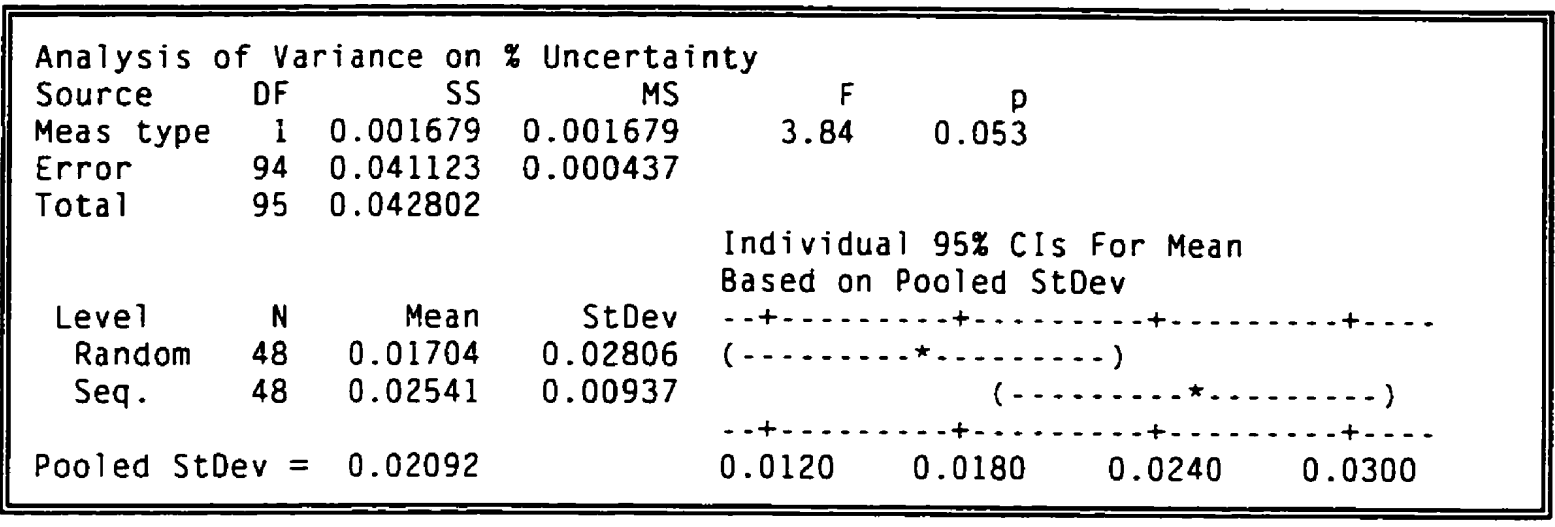

In this analysis, it has been found that the uncertainty estimated by the repeated measures on sample Saskatchewan \# 10 adequately represents the actual uncertainty for all experiments on different sample types and material roughnesses. It also overestimates the uncertainty which would be evident in a truly random experiment. This repeated measures uncertainty based on sample Saskatchewan \#10 will be used for all representation of the data in this experiment. 


\section{Reflection Properties Experiment Results}

The reflection properties experiment was carried out as prescribed in the experimental design. Each of the twenty chosen samples was tested across the entire BRDF. Early results of this experiment were reported in Gibbons and Adrian [1994]. These results led to a redesign of the goniophotometer and an improved control of the measurements systems. The results of the improved system were then reported in Gibbons and Adrian [1996]. This data was compared to the earlier $\alpha=1^{\circ}$ results from the University of Toronto. The data was also analyzed in terms of the interactions between the various angles of incidence and observation. In this chapter, particular attention will be paid to the influence of the change in observation angle on the reflection results. The final aspect of the data analysis will be an evaluation of the various roadway classification systems in terms of the BRDF results.

\subsection{Comparison to University of Toronto}

The first aspect of the test results investigated was the repeatability of the $q_{0}, S 1$ and S2 calculations for each sample. The results of the calculations, in comparison with the data of the University of Toronto are shown in Figure 25. For this comparison, the $\alpha=1^{\circ}$ data from this experiment was compared to the $\alpha=1^{\circ}$ data from the University of Toronto experiment. 


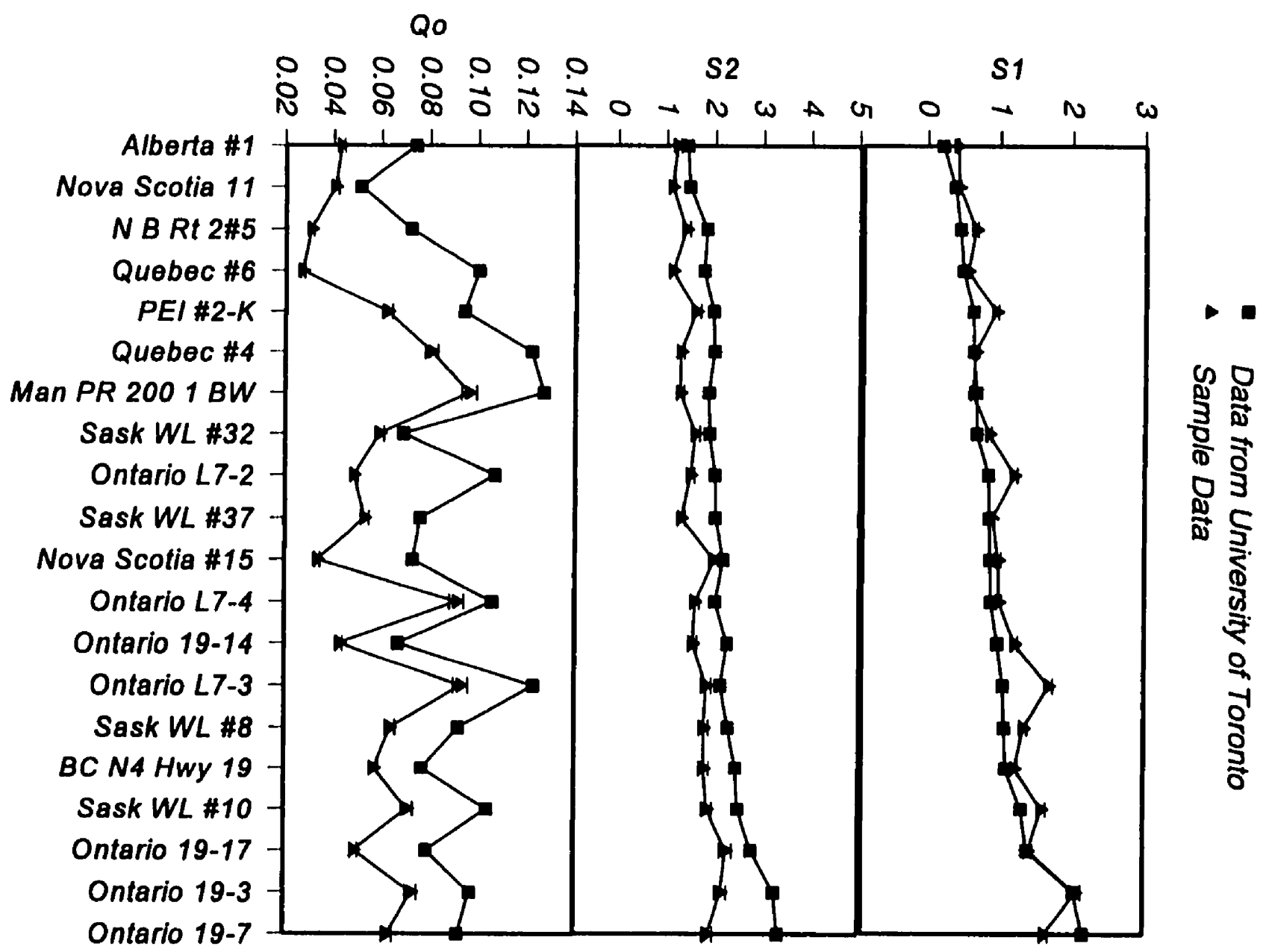


coefficient for this factor is 0.80 . The $\mathrm{q}_{0}$ data does not match the data provided by the University of Toronto as closely. The $r^{2}$ correlation for this data is 0.49 . The $q_{0}$ data for the higher values of S1 do appear to be more closely related. Using the data from samples with $\mathrm{S} 1$ values higher than that for sample Ontario $\mathrm{L} 7-4$, the correlation coefficient changes to $r^{2}=0.86$. The $\mathrm{S} 2$ correlation coefficient is 0.35 . S2 depends on both the calculation of $q_{0}$ and the $r$ values. The $S 2$ error is a combination of the error in both of these factors.

A comparison of the actual reflection data of this experiment to the results of the University of Toronto was performed for samples Saskatchewan \#10 and Ontario L7-2. The results for the change in $\gamma$ and $\beta$ for $\alpha=1^{\circ}$ were investigated. The correlation is generally the same for both samples. The impact of the change in $\gamma$ for sample Ontario L7-2 is shown in Figure 26 and the impact of the change in $\beta$ for sample Saskatchewan \#10 is shown in Figure 27.

It can be seen in this comparison that the best correlation between these two data sets is at high $\beta$ and $\gamma$. A full regression analysis was not possible since the measurement points in the two experiments were not the same. This comparison uses an observation angle of $\alpha=1^{\circ}$ which is the lowest angle of measurement on this experiment's gonioreflectometer. Although the uncertainty at this low angle is not significantly greater than at other angles, the positioning of the detector and the sample are critical at this value. As stated earlier, the gonioreflectometer was designed so that the acceptance angle of the 
luminance meter and the sample size determined the length of the meter support arm. Similarly, the sample was leveled and the height adjusted visually using a bubble level, support wedges and by viewing through the luminance meter optics. This adjustment makes the possibility for inaccuracy from sample to sample very high. In the University of Toronto system, the geometry of the $\alpha$ angle was set permanently, thus removing the variability in the positioning of the luminance meter. The effect of these differences in the measurement systems is most significant at the $\beta=0^{\circ}$ location where the detector is exactly opposite the light source. This inaccuracy in positioning was also likely the source of the differences in the $\mathrm{q}_{0}$ values. To account for this difference, when analysis was performed, the data was scaled by $q_{0}$ to the $q_{0}$ values found by University of Toronto. 


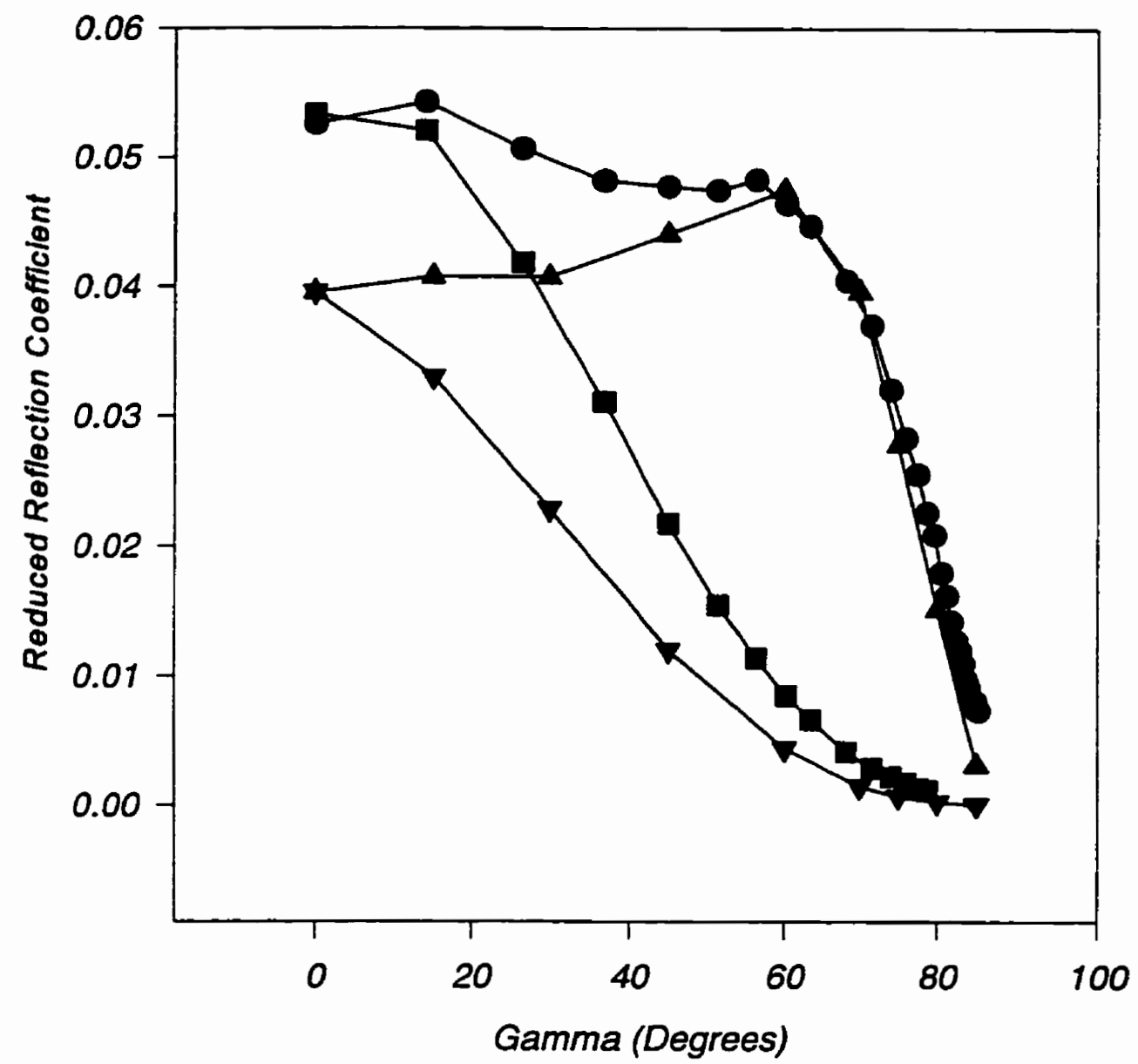

- Ont L7-2-Beta $0^{\circ}-U T \quad \Delta \quad$ Ont L7-2-Beta $0^{\circ}$ UW

Figure 26 - Comparison of the Influence of $\gamma$ from the University of Toronto and this Research (UW) for Sample Ontario L7-2 


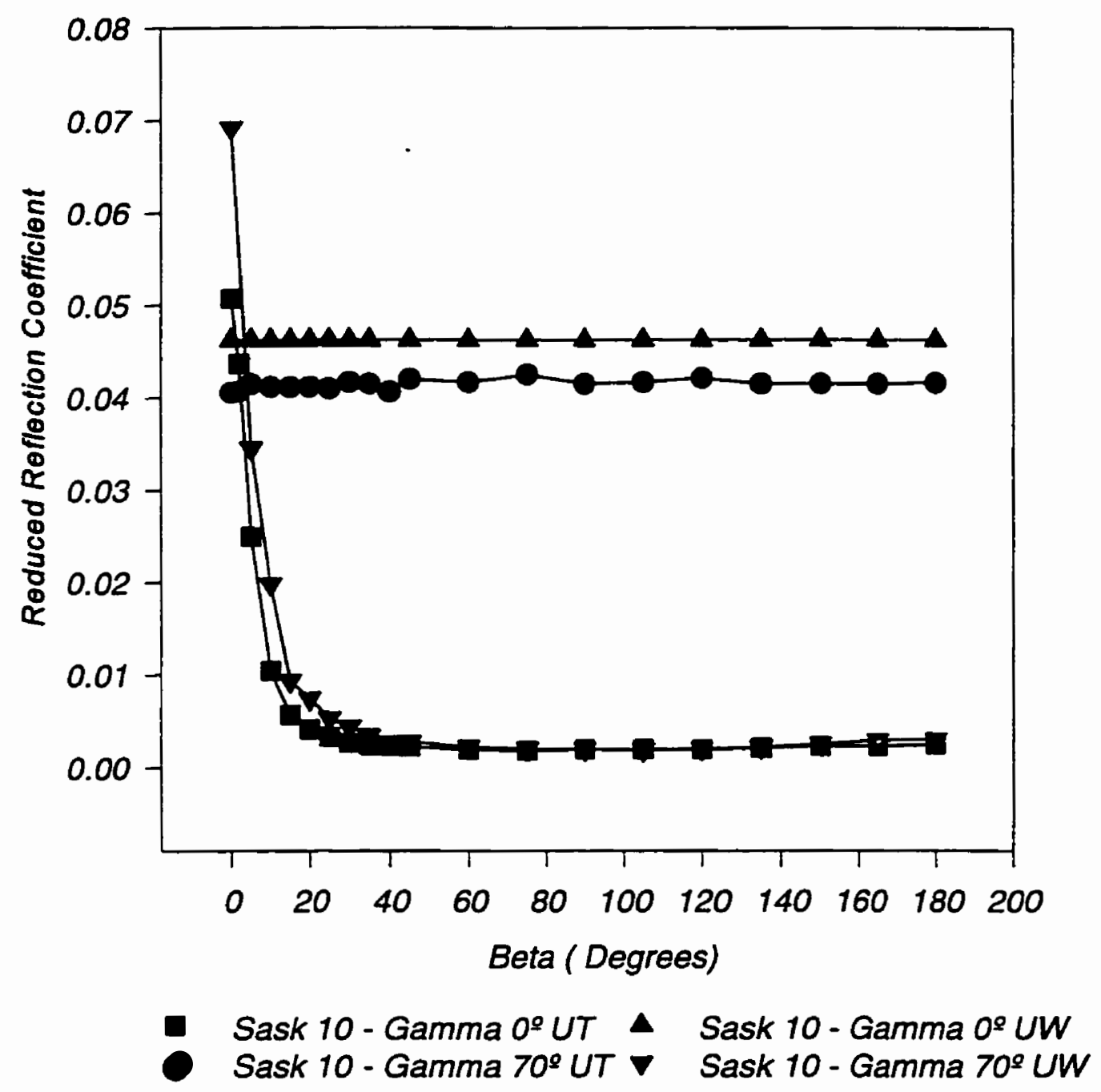

Figure 27 - Comparison of the Change in $\beta$ Results from the University of Toronto Data to this Research (UW) for Sample Saskatchewan 10

\subsection{Influence of Incidence and Observation Angles}

The impact of the change in observation angle on the reflection factor, $q$, is shown in

Figures 28 through 31 . Each figure shows the results for each sample in an R-Class. The 
figures show the average change in observation angle for all incident angles. The data has all been scaled to a standard illumination level of $80 \mathrm{~lx}$ and by $q_{0}$.

The most noticeable aspect of this data is the apparent different relative leveis of reflectance for some of the samples. The standout of these samples is Quebec \#6 from the R2 Class. This sample has a very low calculated $q_{0}$ as compared to the results of University of Toronto results. The scaling by the small value of $q_{0}$ for this sample appears to result in too large a reflectance value. It should be noted that the scaling is apparently correct for the $\alpha=1^{\circ}$ data point as it falls in line with the other $\alpha=1^{\circ}$ data points. This effect is also seen in the results for the Nova Scotia \#15 sample. 


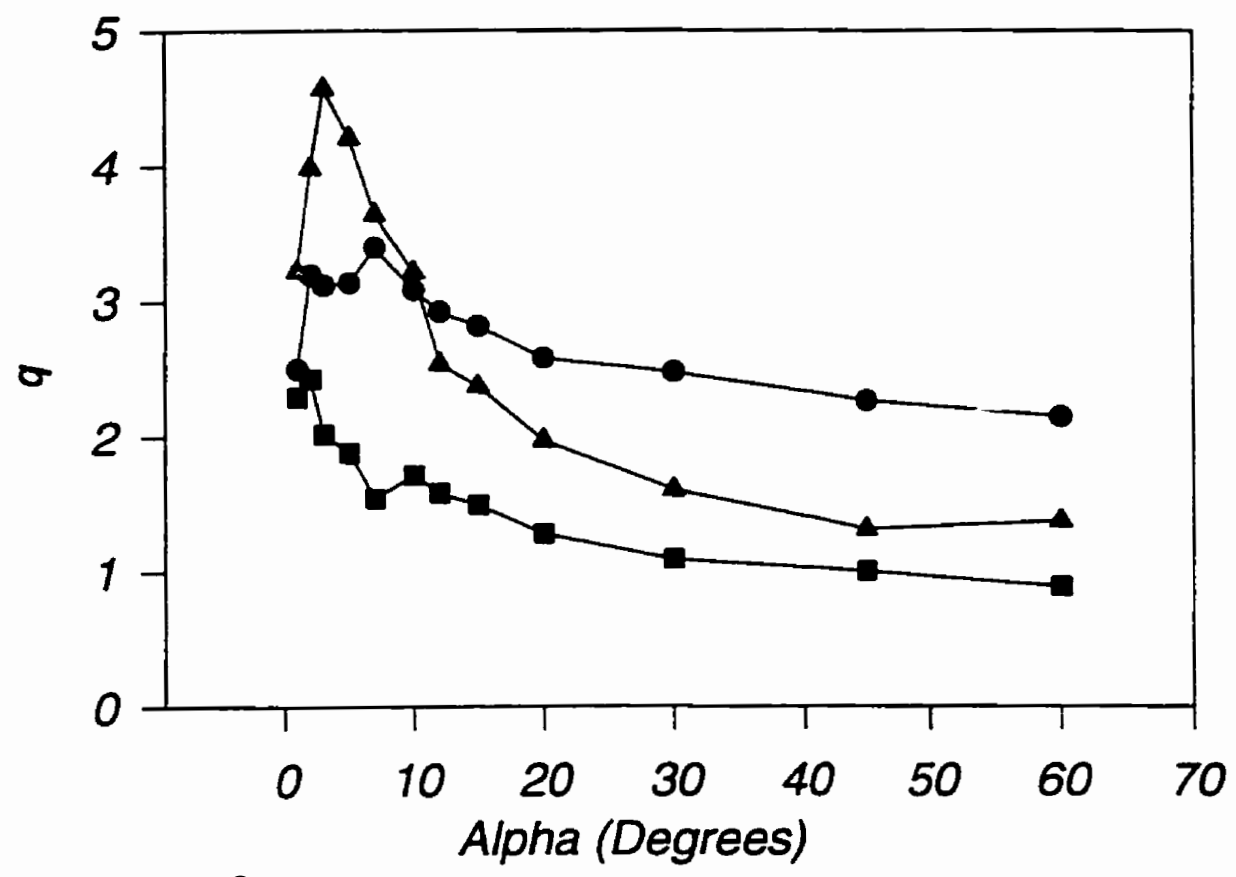

Alberta\#1 4 Nova Scotia 11 New Brunswick Rt2 \#5

Figure 28 - Influence of $\alpha$ on the Reflection Profile for RI Class Samples 


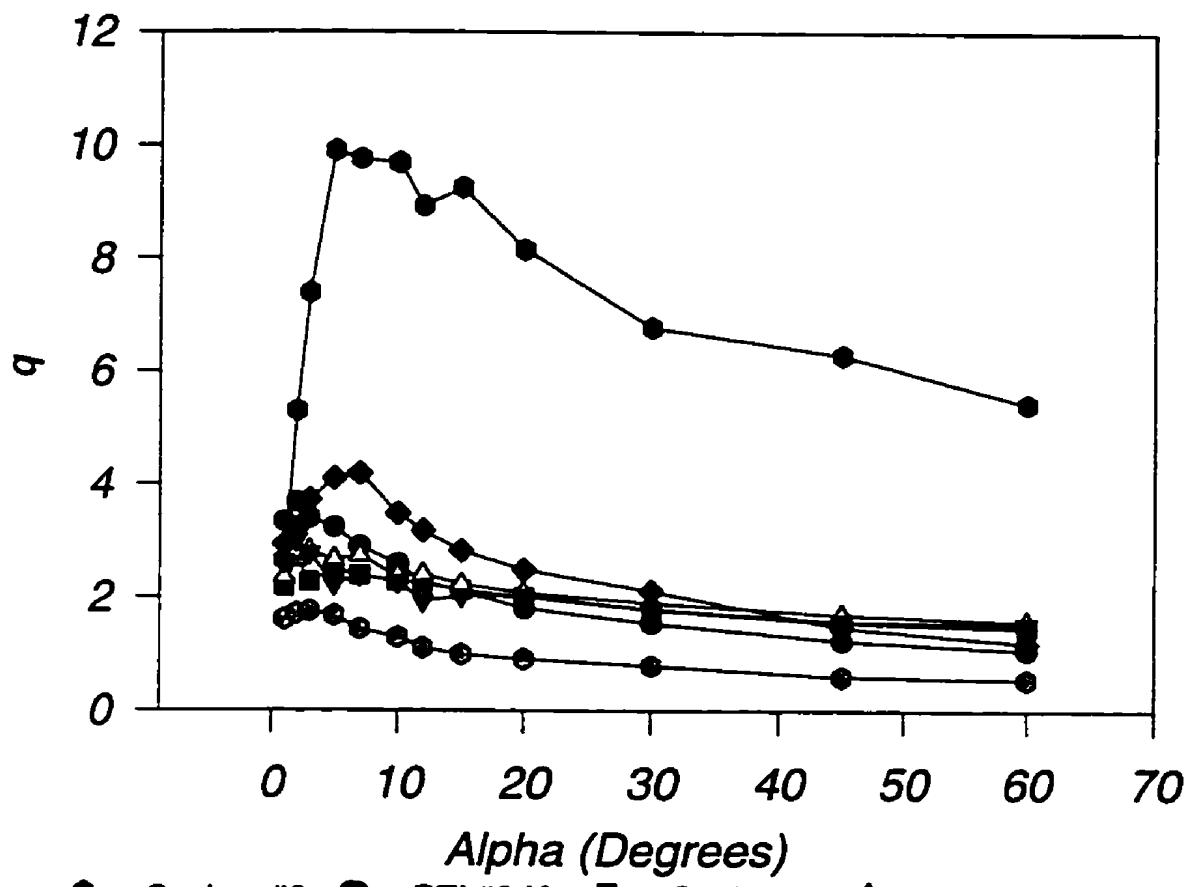

- Quebec \#6 - PEI \#2-K Q Quebec \#4 $\triangle$ Man PR 2001 BWP

$\nabla$ Sask WL \#32 Ontario L7-2 - Sask WL \#37

Figure 29 - Influence of $\alpha$ on the Reflection Profile for R2 Class Samples 


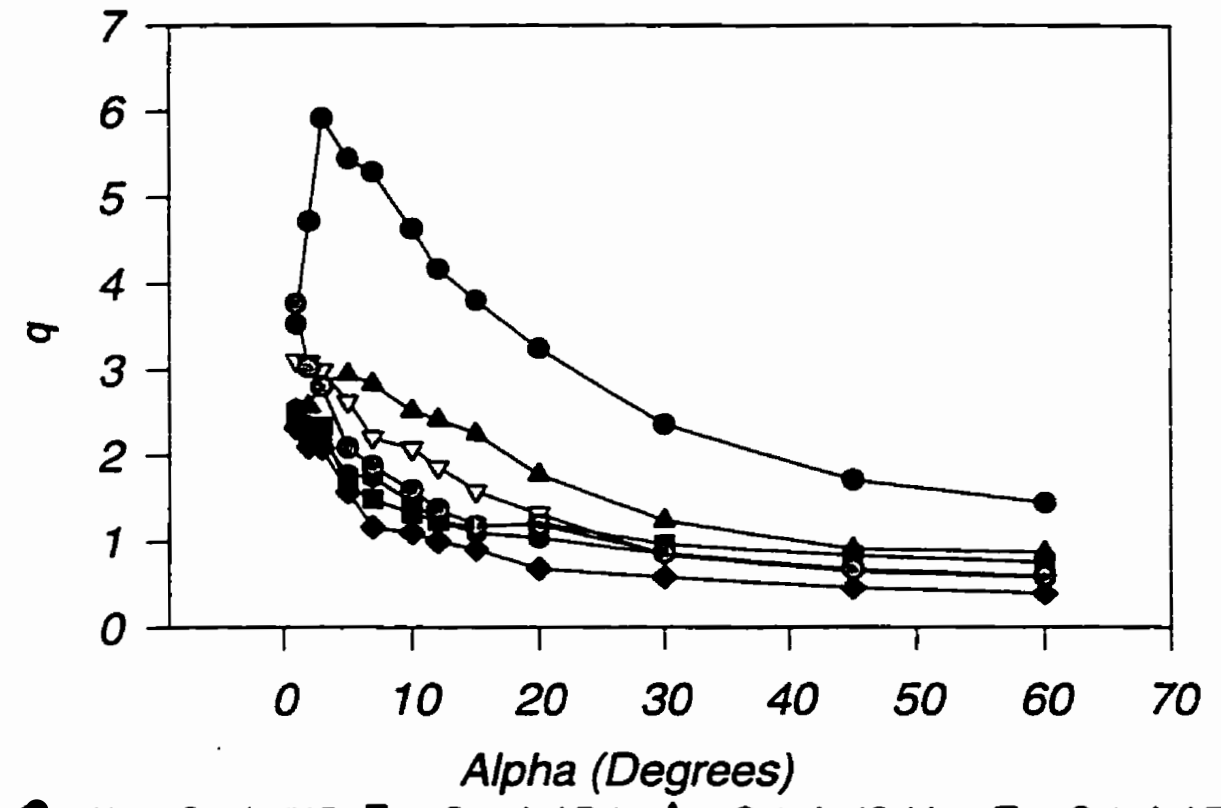

- Nova Scotia \#15 Ontario L7-4 $\triangle$ Ontario 19-14 $\nabla$ Ontario L7-3

Sask WL\#8 BCN4HWy $19 \odot$ Sask WL\#10

Figure 30 - Influence of $\alpha$ on the Reflection Profile for R3 Class Samples 


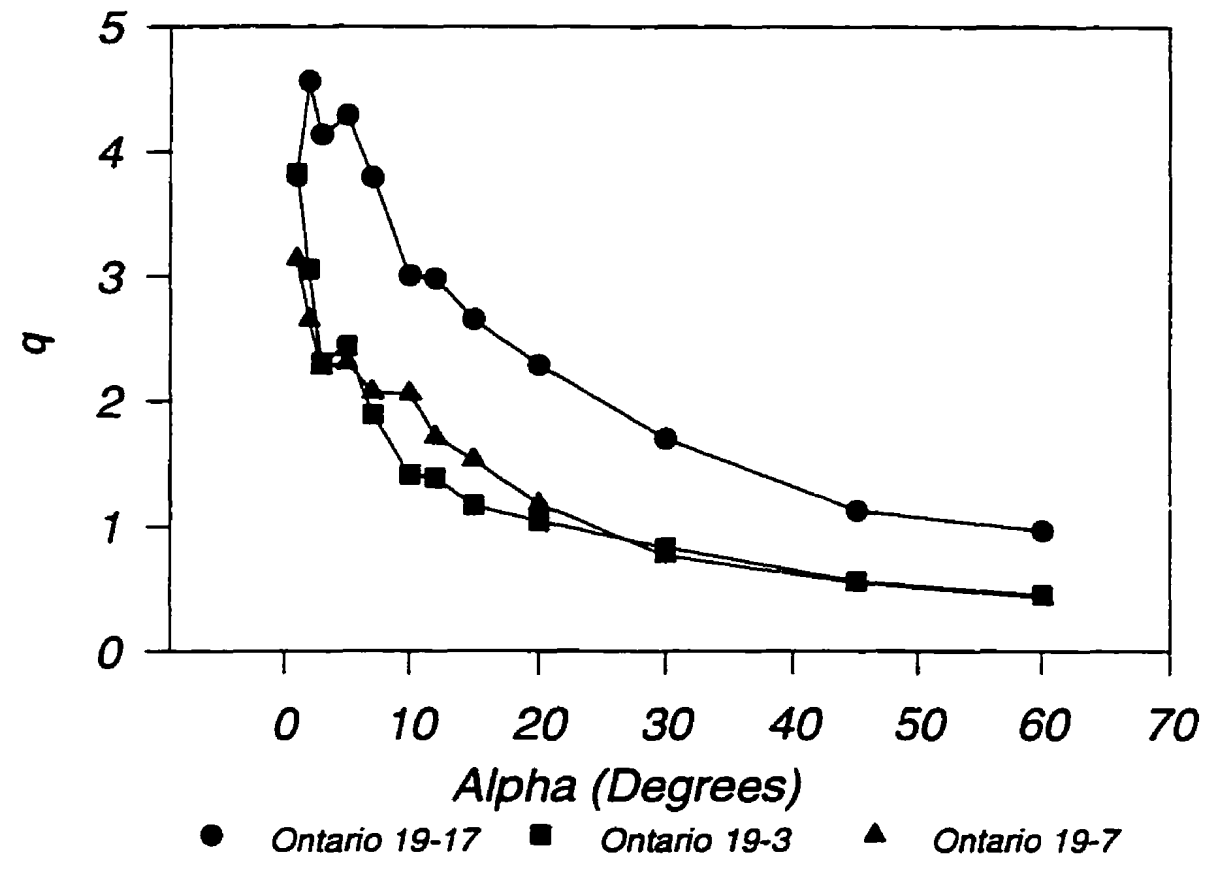

Figure 31 - Influence of $\alpha$ on the Reflection Profile for R4 Class Samples

The influence of the rotational incident angle, $\beta$, is shown in Figures 32 to 35 for each $R$ Class. Like the previous group of figures, this data has been scaled to a standard illuminance of $80 \mathrm{~lx}$. The effect of the scaling on the Quebec \#6 sample is evident in these results. 


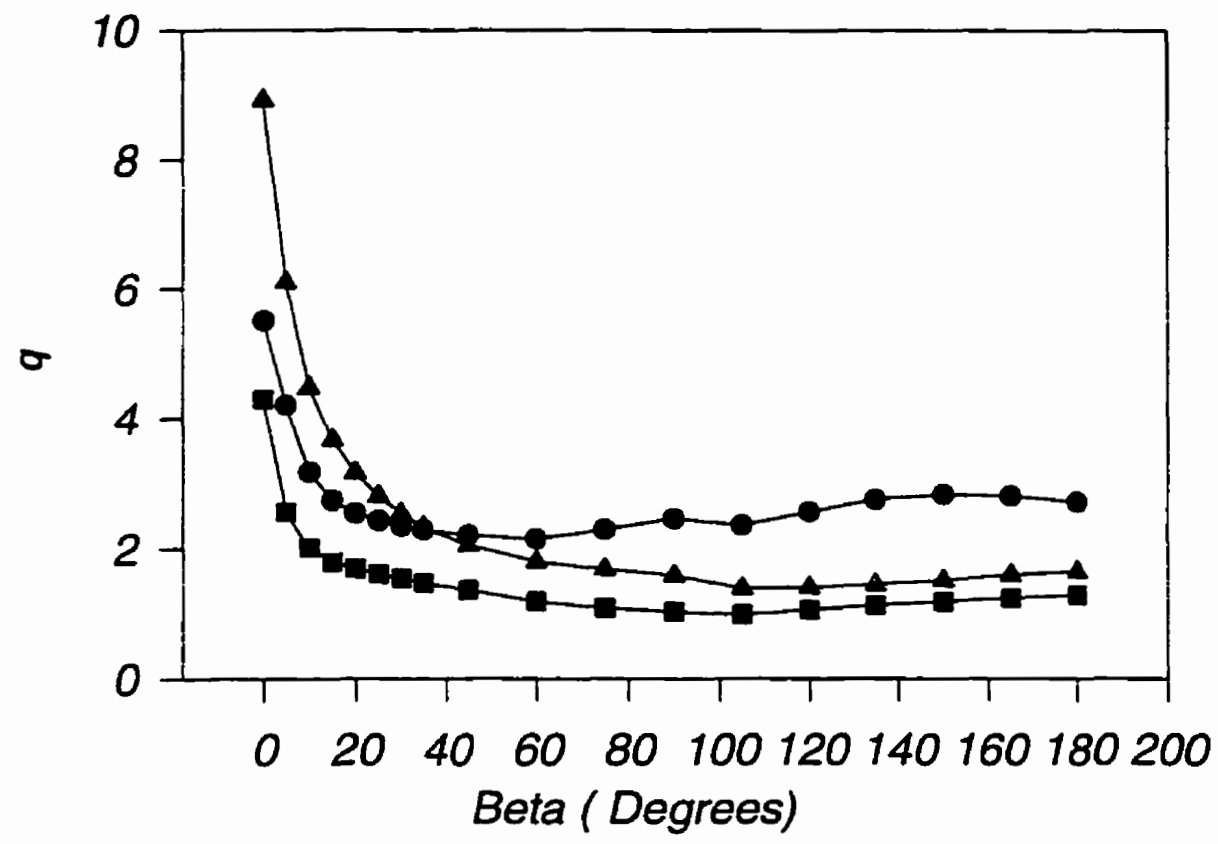

- Alberta \#1 - Nova Scotia 11 - New Brunswick Rt2 \#5

Figure 32 - Influence of $\beta$ on the Reflection Profile for RI Class Samples 


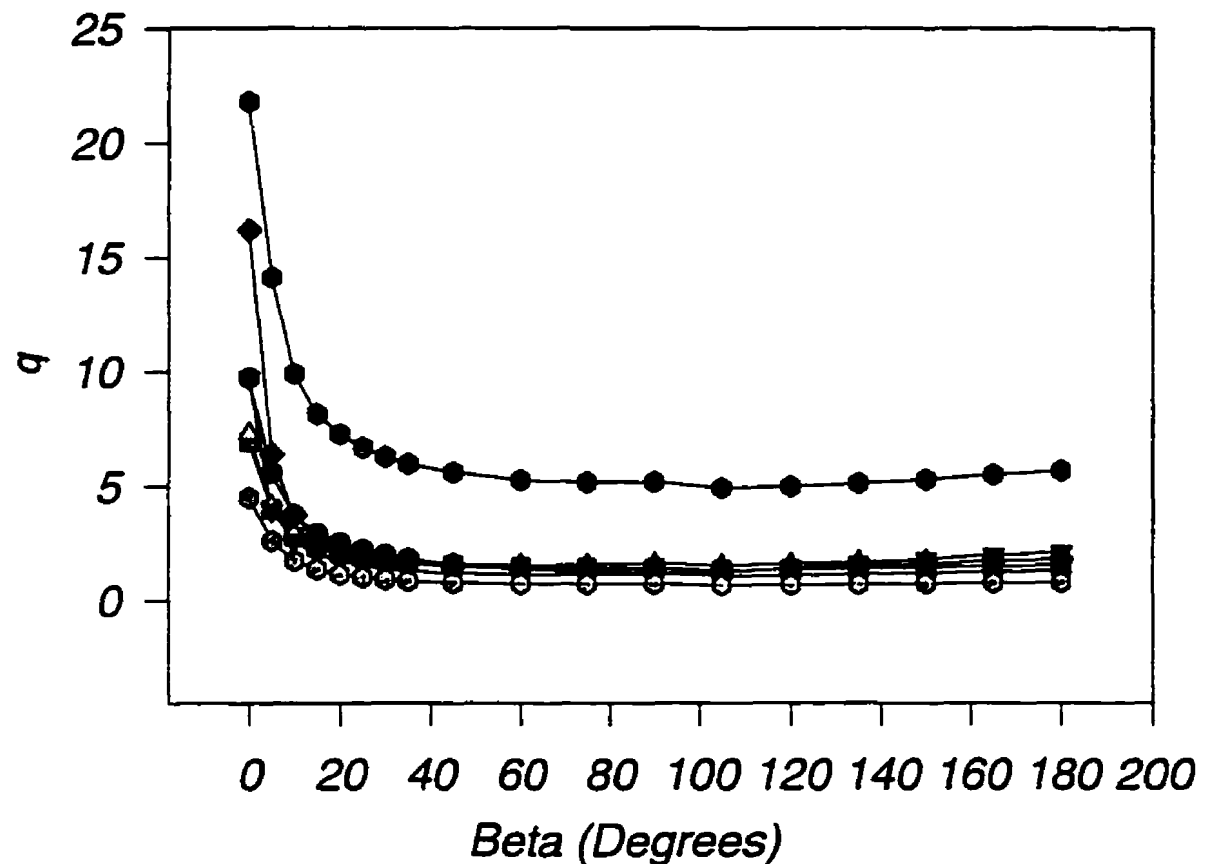

- Quebec \#6 - PEI \#2-K - Quebec \#4 $\triangle$ Man PR2001 BWP

$\nabla$ Sask WL \#32 - Ontario L7-2 ○ Sask WL \#37

Figure 33 - Influence of $\beta$ on the Reflection Profile for R2 Class Samples 


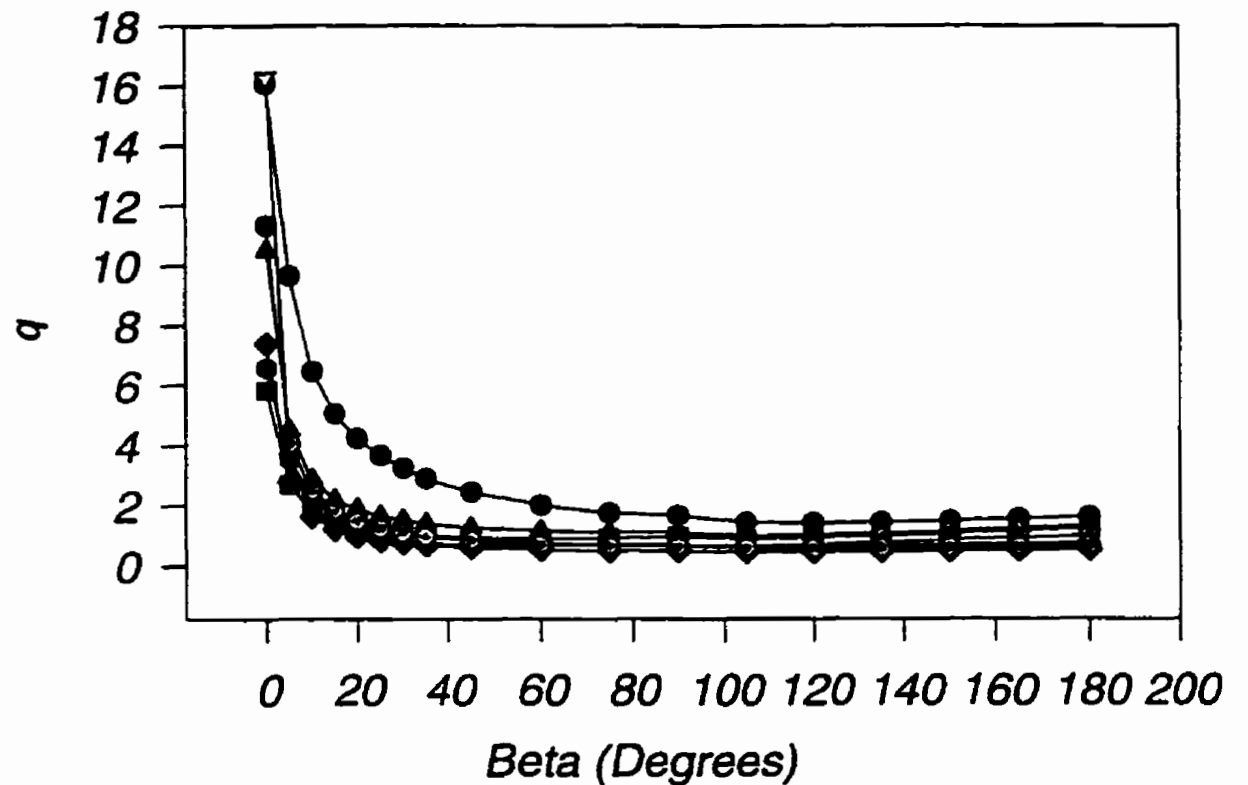

- Nova Scotia \#15 Ontario L7-4 \ Ontario 19-14 $\nabla$ Ontario L7-3 - Sask WL \#8 BC N4 Hwy $19 \bigcirc$ Sask WL \#10

Figure 34 - Influence of $\beta$ on the Reflection Profile for R3 Class Samples 


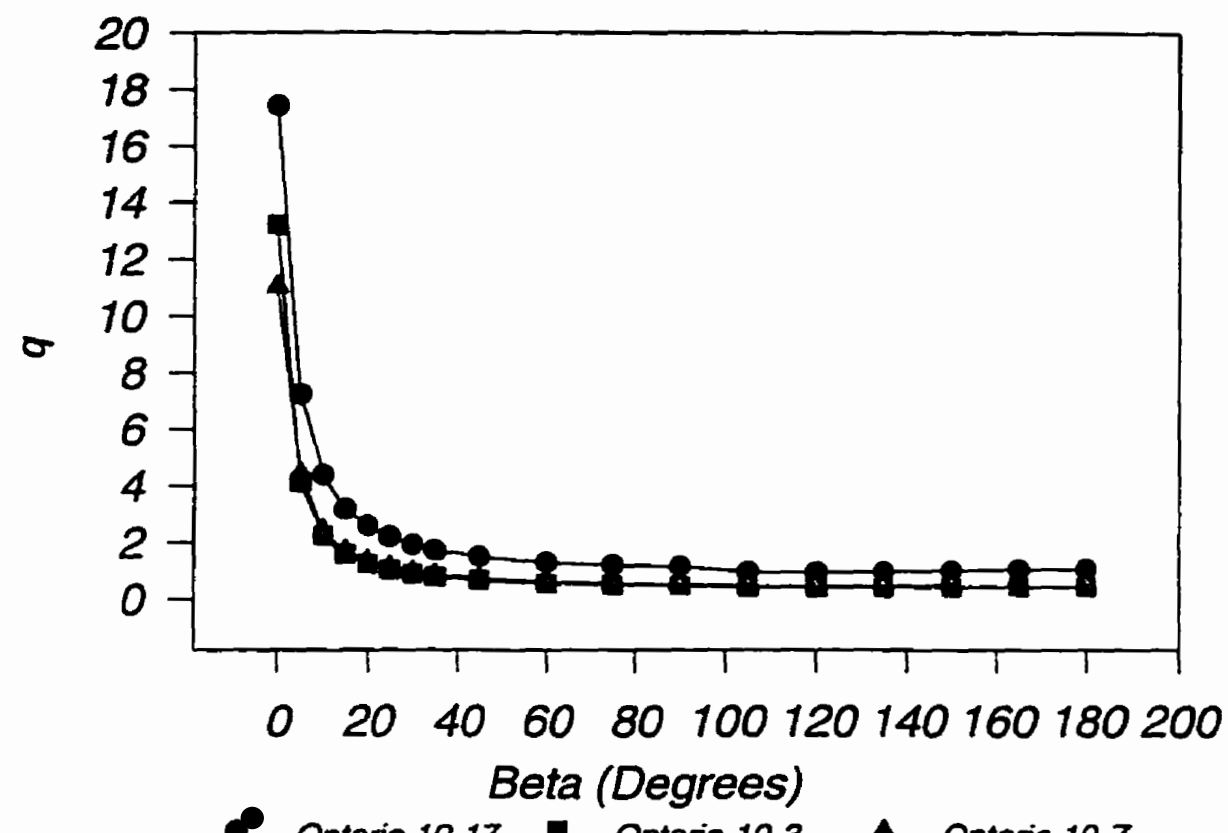

Figure 35 - Influence of $\beta$ on the Reflection Profile of R4 Class Pavements

The influence of the altitude incident angle, $\gamma$, is shown in Figures 36 to 39 for each $R$ -

Class. Like the two previous figure sets, the data has been scaled to a standard

illuminance of $80 \mathrm{~lx}$. Again, the Quebec \#6 sample shows numerically higher results than the others in the same class. 


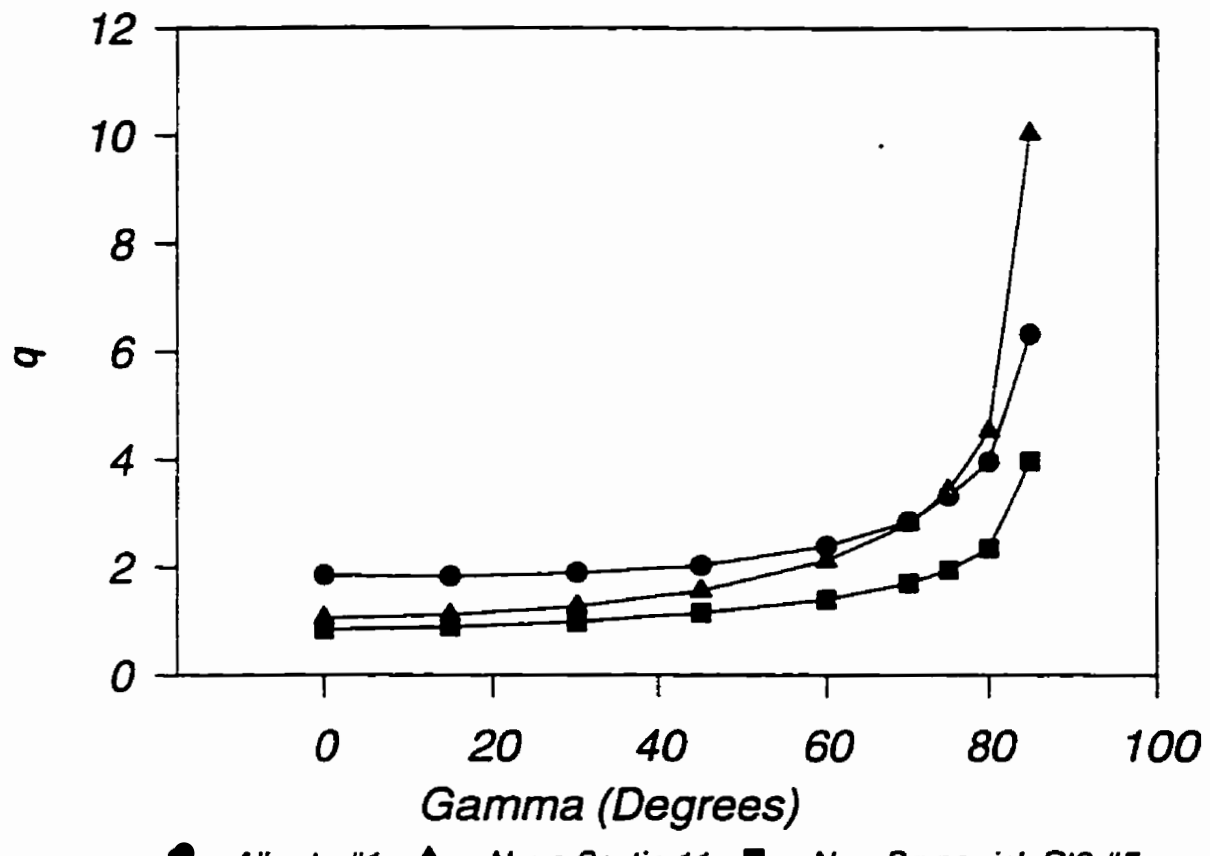

Figure 36 - Influence of $\gamma$ on the Reflection Profile for RI Class Samples 


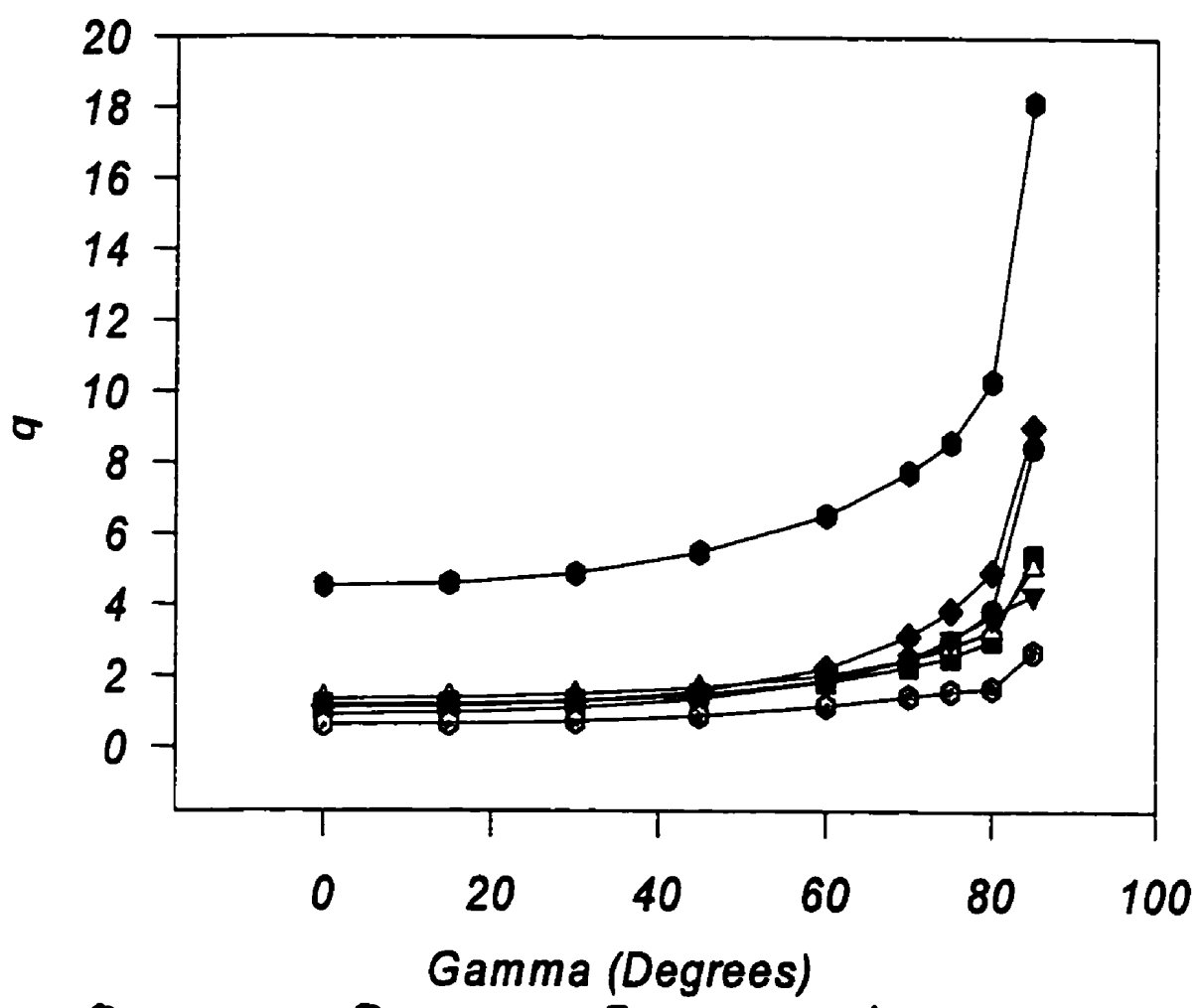

- Quebec \#6 PEI\#2-K Quebec \#4 $\triangle$ Man PR 2001 BWP

$\nabla$ Sask WL \#32 Ontario L7-2 Sask WL \#37

Figure 37 - Influence of $\gamma$ on the Reflection Profile for R2 Class Samples 


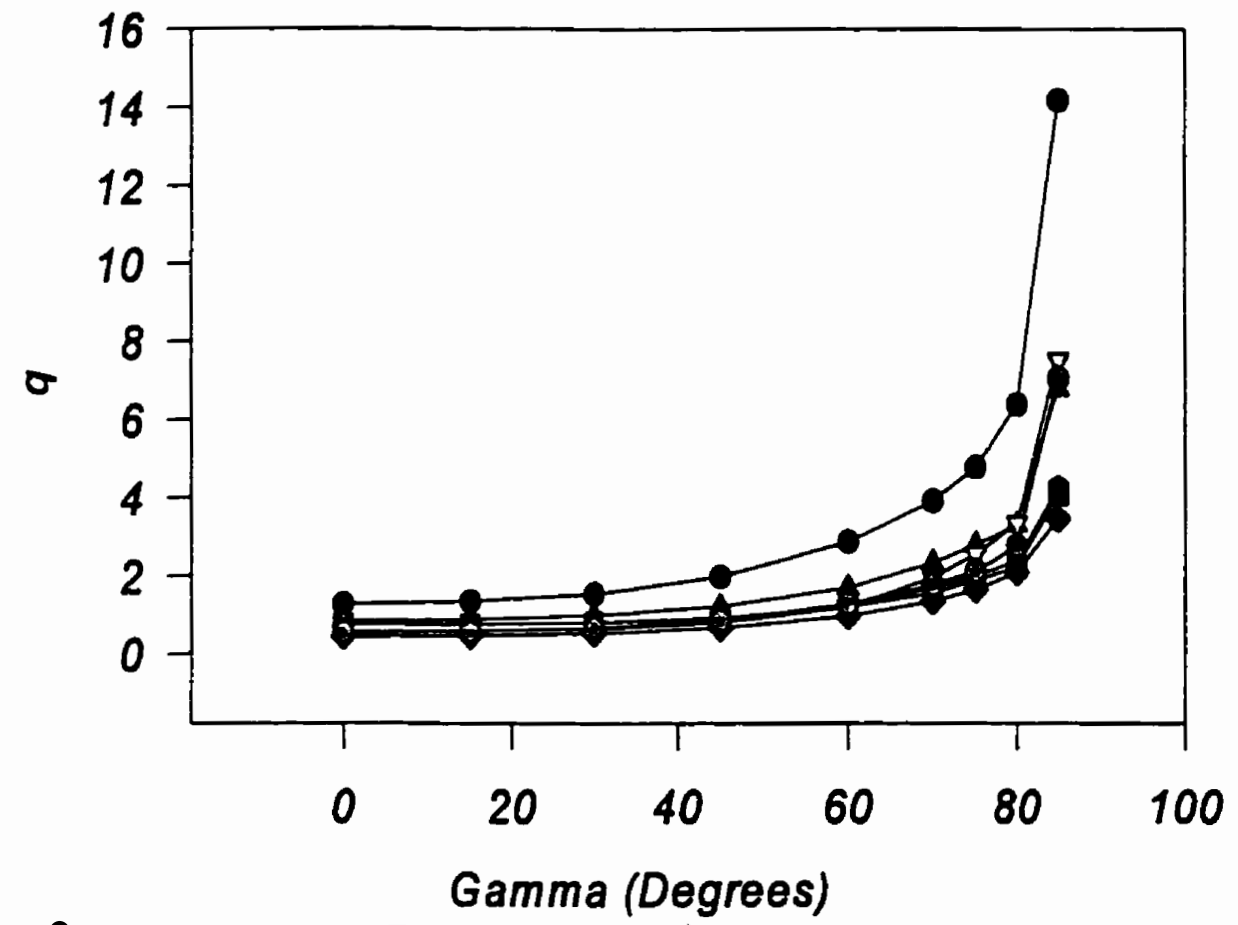

- Nova Scotia \#15 - Ontario L7-4 A Ontario 19-14 $\nabla$ Ontario L7-3

Sask WL \#8 BC N4 HWy $19 \bigcirc$ Sask WL \#10

Figure 38 - Influence of $\gamma$ on the Reflection Profile for R3 Class Samples 


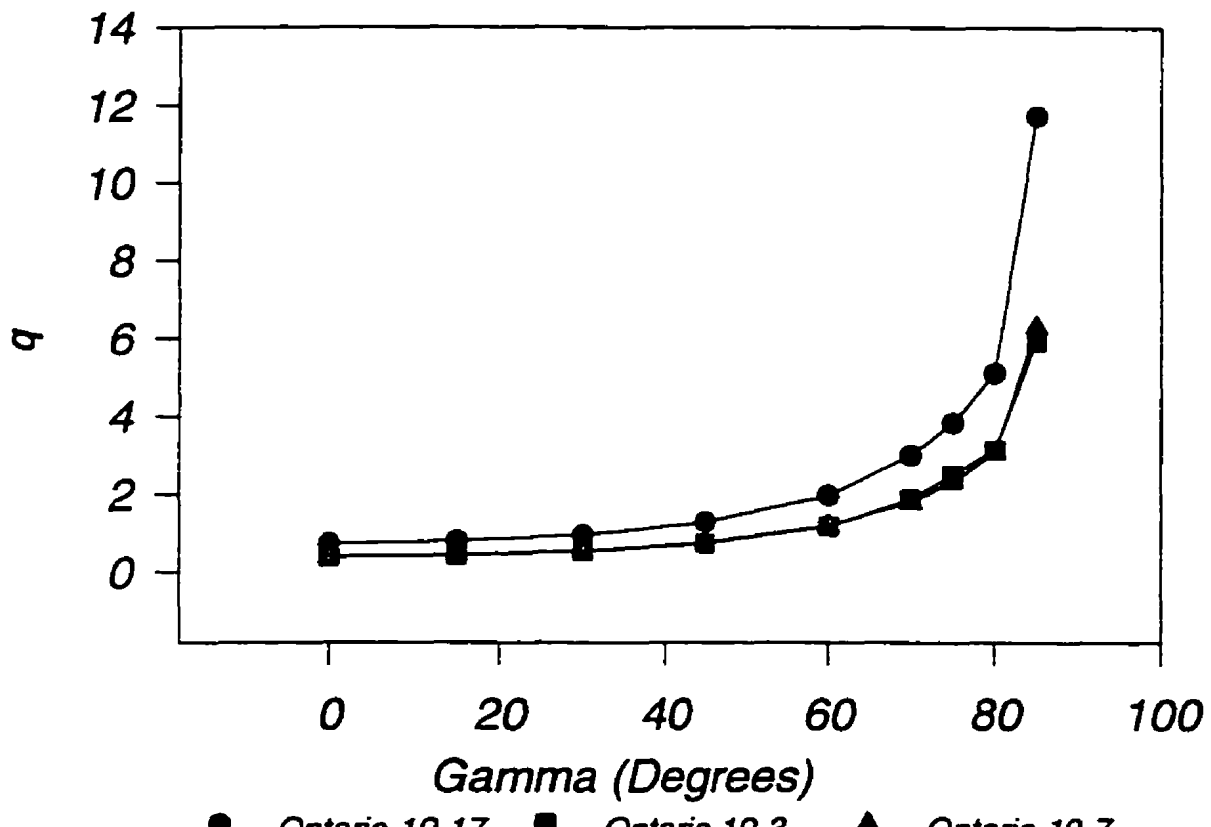

Figure 39 - Influence of $\gamma$ on the Reflection Profile of R4 Class Samples

Due to the number of measurements made for each sample and the number of samples used, the data will generally be represented using graphs of the averages across the road classes as defined by the current CIE system. The influence of all of the observation and incident angles for each road class is shown in Figures 40 to 42 . For these graphs, sample Quebec \#6 has been left out of the average due to the obvious non-compliance of this scaled data set to the other samples in the road class. 


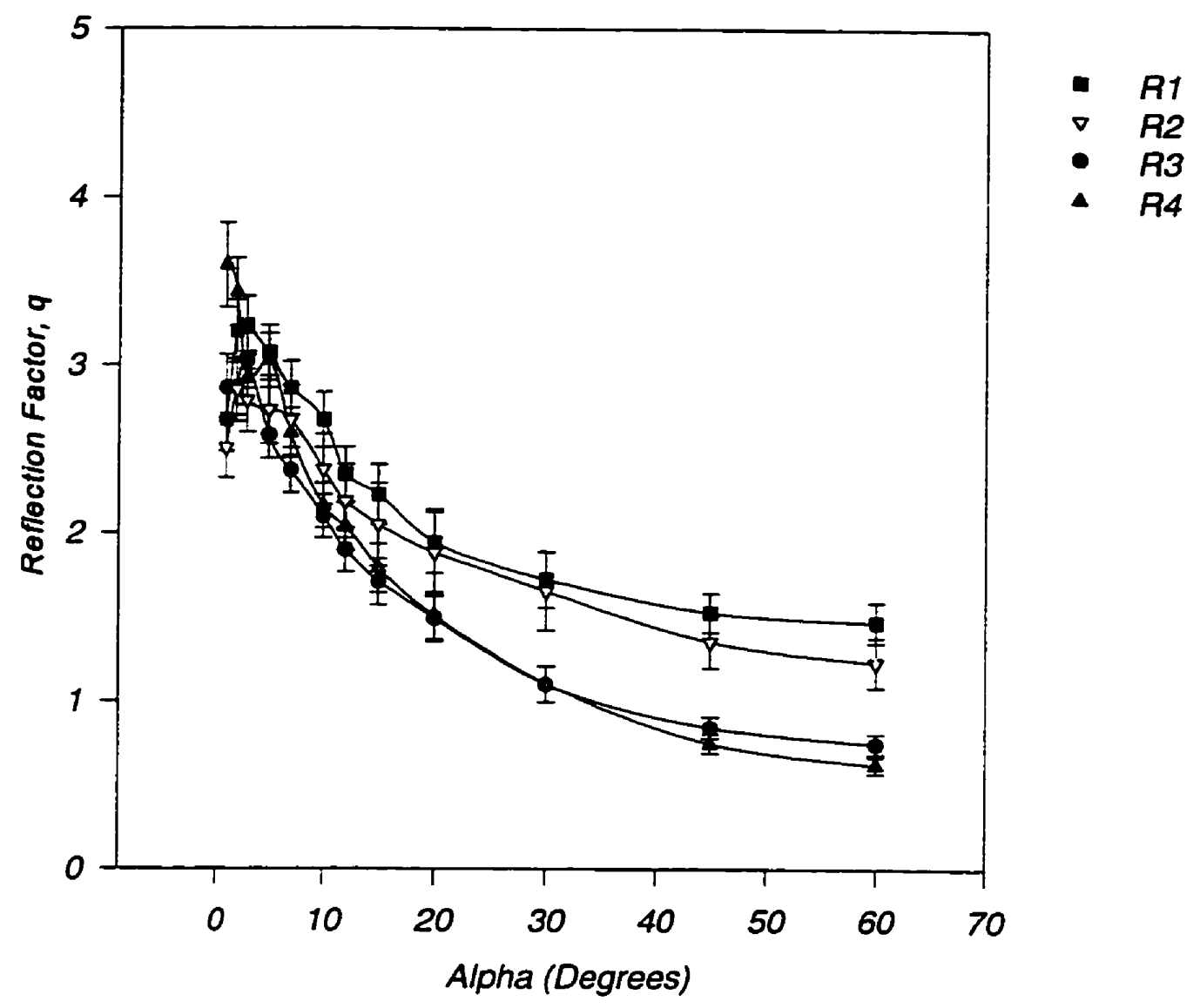

Figure 40 - Influence of $\alpha$ for All R-Classes 


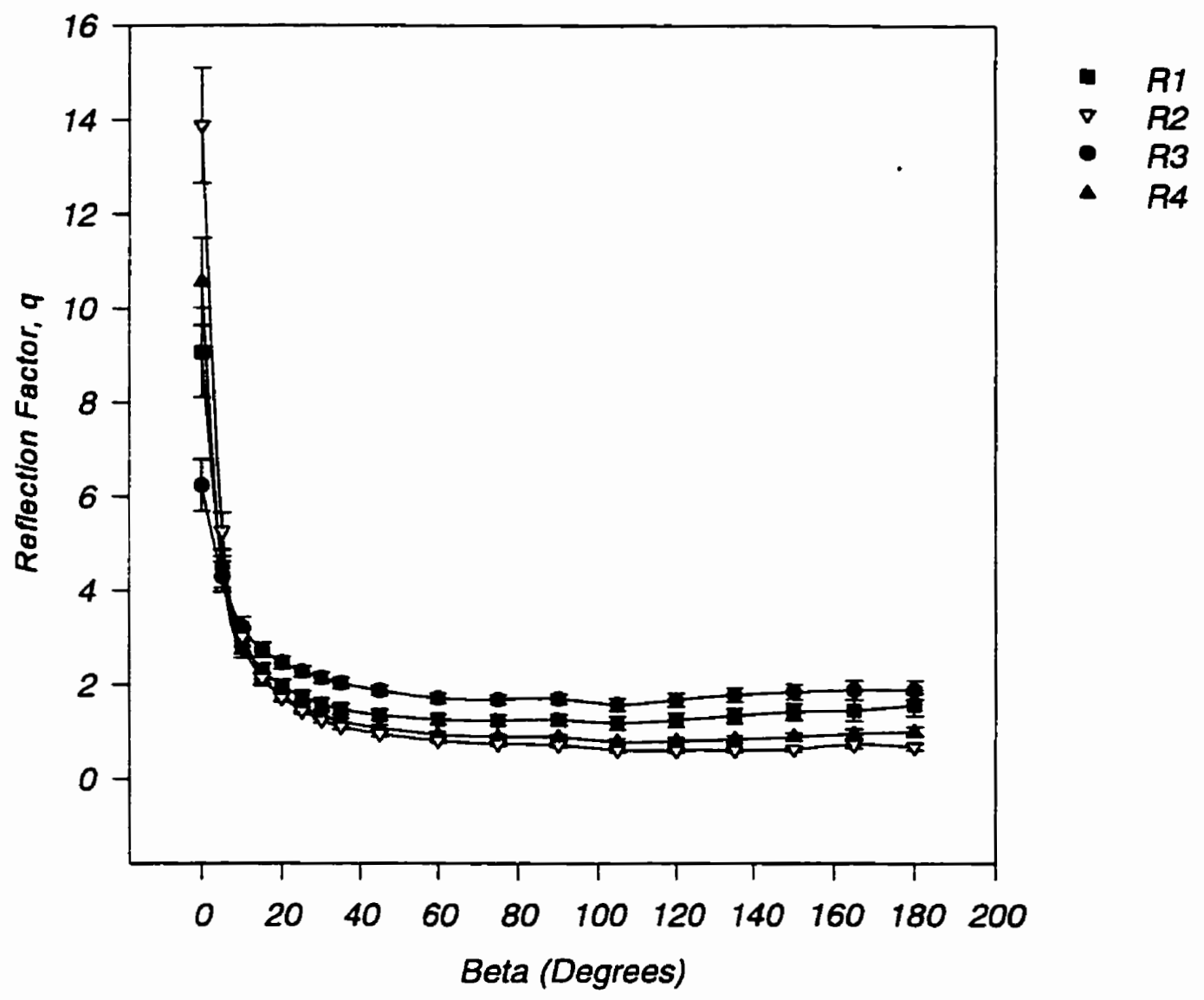

Figure 41 - Influence of $\beta$ for All R-Classes 


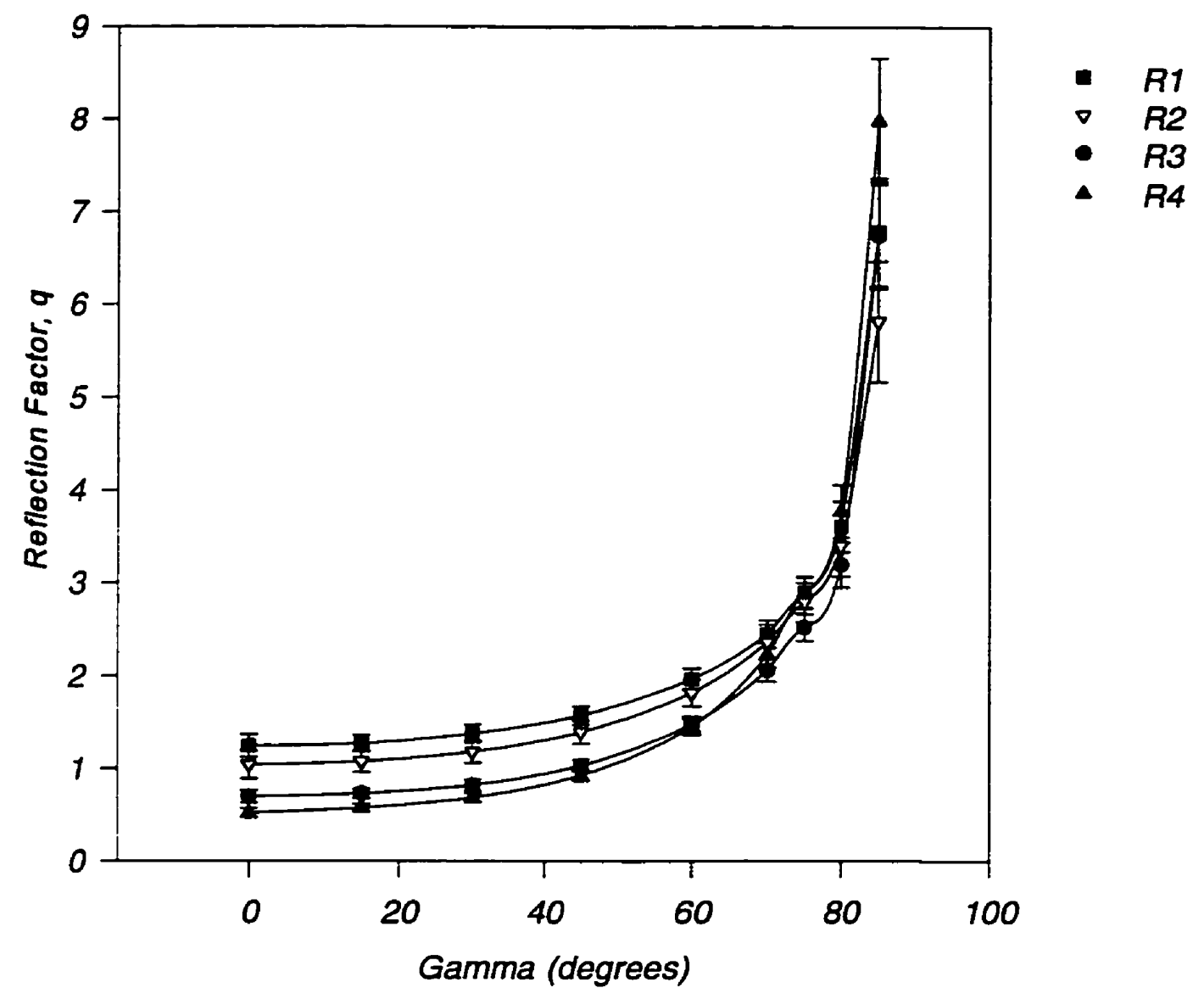

Figure 42 - Influence of $\gamma$ for All R-Classes

The trend shown in all of these figures is a trend of the data towards a horizontal line.

This means that the reflectivity of the sample becomes less related to the angle of interest. As shown in Chapter 3, the reflection mode which is not related to the angle of observation is Lambertian or diffuse reflection. Based on this data, the reflectivity of the sample becomes diffuse at high $\beta$ and low $\gamma$ and close to diffuse for higher $\alpha$. 


\section{$5.3 \alpha, \beta$, and $y$ Interactions}

In order to establish the nature of the reflection profile in terms of the various

measurement parameters, the interactions of the variables were studied using Analyses of

Variance performed on the data set. The ANOVA was performed on the reflectivity, q,

for the entire data. The results for the angular measurements are shown in Table 12.

Table 12 - ANOVA for $\alpha, \beta$, and $\gamma$ Interactions

\begin{tabular}{|c|c|c|c|c|c|c|}
\hline \multicolumn{7}{|c|}{ Analysis of Variance for 0} \\
\hline Source & DF & Seq $S S$ & $\operatorname{Adj} \mathrm{SS}$ & Adj MS & $F$ & $\mathrm{p}$ \\
\hline Beta & 11 & 32267.8 & 32267.8 & 2933.4 & 497.73 & 0.000 \\
\hline Gamma & 8 & 19847.8 & 19847.8 & 2481.0 & 420.96 & 0.000 \\
\hline Alpha & 11 & 4829.5 & 4829.5 & 439.0 & 74.50 & 0.000 \\
\hline Bet $a \star G a m m a$ & 88 & 55278.7 & 55278.7 & 628.2 & 106.58 & 0.000 \\
\hline Beta*Alpha & 121 & 21139.3 & 21139.3 & 174.7 & 29.64 & 0.000 \\
\hline Gamma*Alpha & 88 & 6490.9 & 6490.9 & 73.8 & 12.52 & 0.000 \\
\hline Be $\tau a^{\star} G a m m a \star A l p h a$ & 968 & 42439.7 & 42439.7 & 43.8 & 7.44 & 0.000 \\
\hline $\begin{array}{l}\text { Error } \\
\text { Total }\end{array}$ & $\begin{array}{l}3888 \\
5183\end{array}$ & $\begin{array}{r}22914.3 \\
205208.0\end{array}$ & 22914.3 & 5.9 & & \\
\hline
\end{tabular}

Table 12 shows that all of the angular parameters and all of their interactions are significant in the experimental results. This result was expected based on the data presented earlier. A sample of this interaction is also shown in Figure 43. 


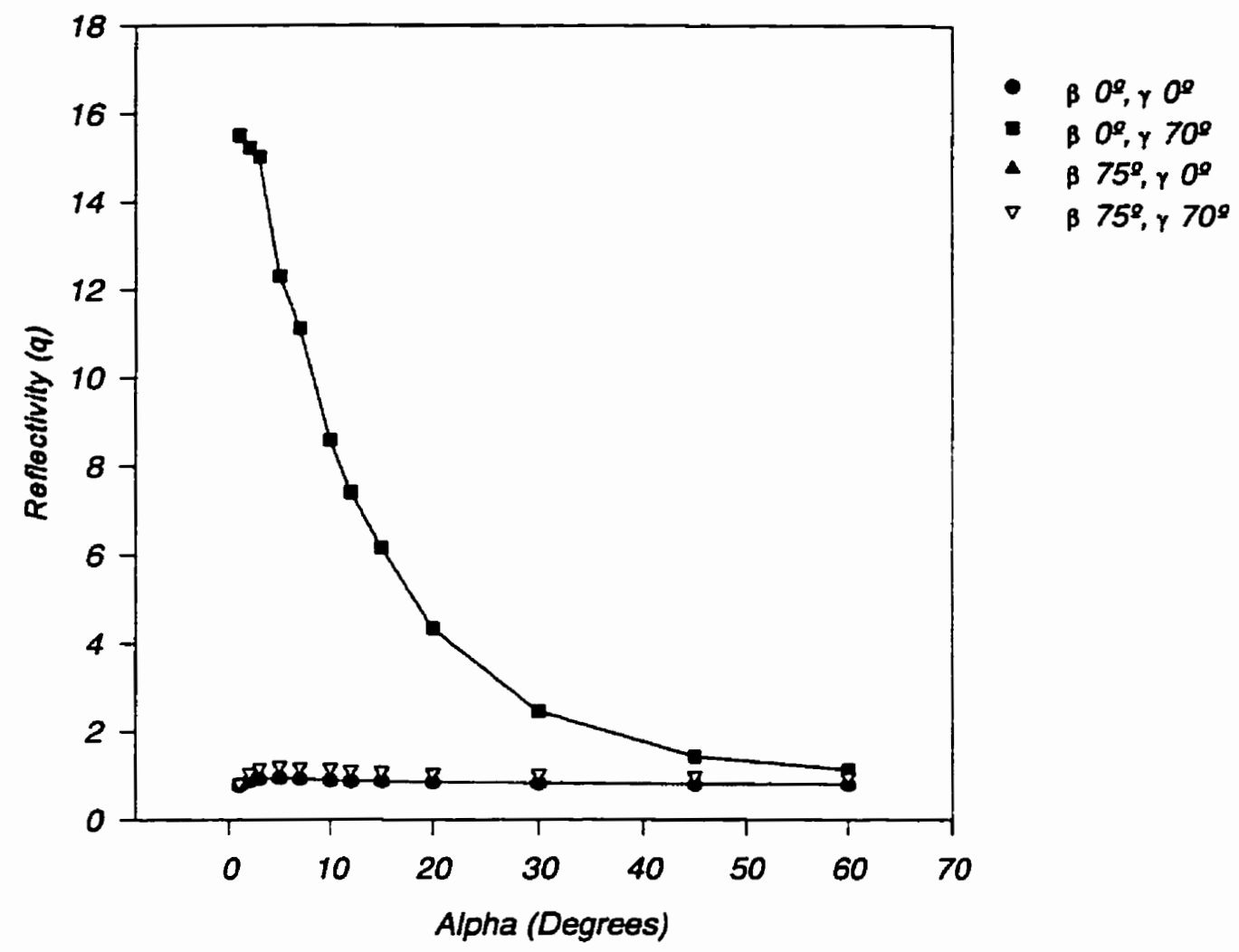

Figure 43 - Interaction of $\alpha, \gamma$ and $\beta$

The experimental results were also analyzed for the sample R-Class. Due to the number of samples, the data for this analysis was averaged across the R-Class. The first ANOVA was performed for the general significance of the R-Class in the data. These results are presented in Table 13. This analysis shows that the R-Class is a significant factor in the reflection data. This was also expected in terms of the data presented earlier. 
Table 13 - ANOVA for R-Class

\begin{tabular}{|lrrrrrr|}
\hline \hline \multicolumn{2}{|l|}{ Analysis of Variance for 0} & & & & \\
Source & DF & Seq SS & Adj SS & Adj MS & $F$ & P \\
R & 3 & 620.58 & 620.58 & 206.86 & 5.24 & 0.001 \\
Error & 5180 & 204587.38 & 204587.38 & 39.50 & & \\
Total & 5183 & 205207.95 & & & & \\
\hline
\end{tabular}

The other interactions investigated were in the $\alpha, \beta$ and $\gamma$ interactions with the R-Class.

These factors were investigated in three separate ANOVAs due to the size and the extensive calculation requirements for the computed statistics. The result of this ANOVA is shown in Tables 14 to 16.

Table 14 - ANOVA for R-Class, $\alpha$, and $\gamma$ Interactions

\begin{tabular}{||lrrrrrr||}
\hline \hline Analysis of Variance for 0 \\
Source & DF & Seq SS & Adj SS & Adj MS & F & P \\
$R$ & 3 & 620.58 & 620.58 & 206.86 & 5.73 & 0.001 \\
Gamma & 8 & 19847.79 & 19847.79 & 2480.97 & 68.74 & 0.000 \\
Alpha & 11 & 4829.51 & 4829.51 & 439.05 & 12.16 & 0.000 \\
$R^{\star}$ Gamma & 24 & 415.55 & 415.55 & 17.31 & 0.48 & 0.984 \\
$R^{\star}$ Alpha & 33 & 657.85 & 657.85 & 19.93 & 0.55 & 0.982 \\
Gamma*Alpha & 88 & 6490.90 & 6490.90 & 73.76 & 2.04 & 0.000 \\
$R^{\star}$ Gamma*Alpha & 264 & 830.90 & 830.90 & 3.15 & 0.09 & 1.000 \\
Error & 4752 & 171514.88 & 171514.88 & 36.09 & & \\
Total & 5183 & 205207.95 & & & & \\
\hline
\end{tabular}


Table 15 - ANOVA for R-Class, $\gamma$ and $\beta$ Interactions

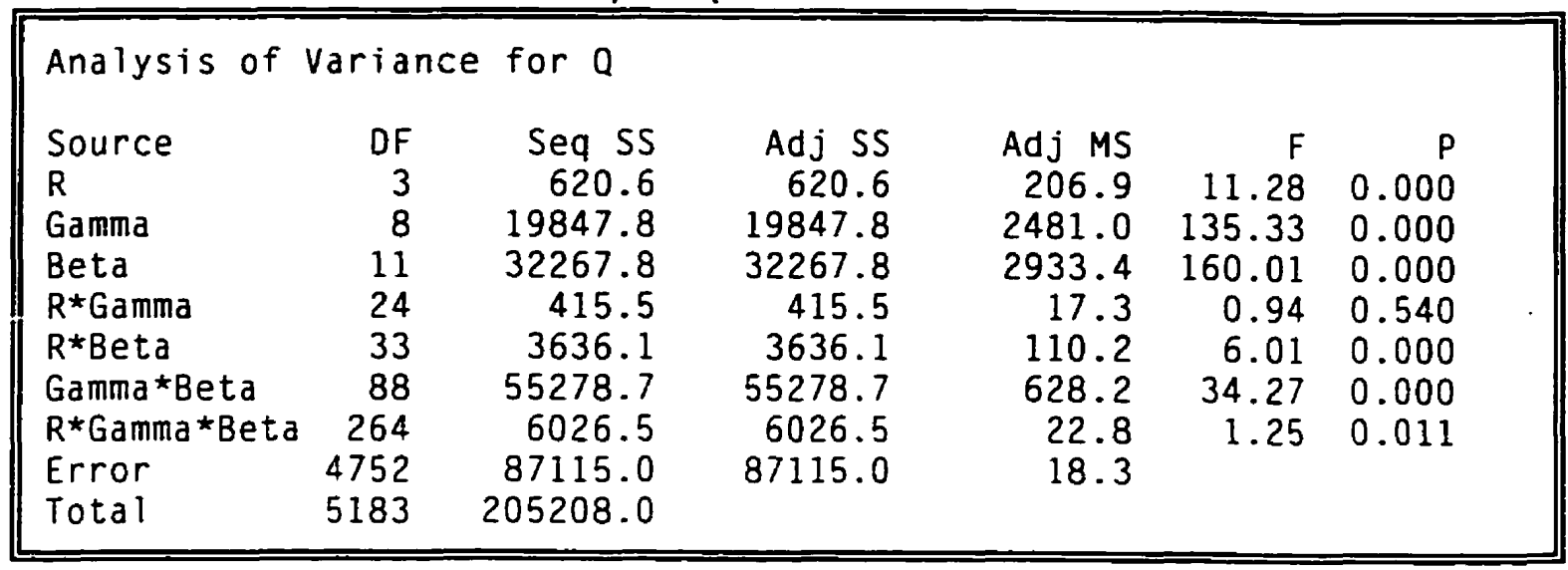

Table 16 - ANOVA for R-Class, $\alpha$ and $\beta$ Interactions

\begin{tabular}{|c|c|c|c|c|c|c|}
\hline \multicolumn{7}{|c|}{ Analysis of Variance for $Q$} \\
\hline $\begin{array}{l}\text { Source } \\
R \\
\text { Beta } \\
\text { Alpha } \\
R \star B e t a \\
R \star A l p h a \\
\text { Beta*Alpha } \\
R \star B e t a \star A l p h a \\
\text { Error } \\
\text { Total }\end{array}$ & $\begin{array}{r}\text { DF } \\
3 \\
11 \\
11 \\
33 \\
33 \\
121 \\
363 \\
4608 \\
5183\end{array}$ & $\begin{array}{r}\text { Seq SS } \\
620.58 \\
32267.79 \\
4829.51 \\
3636.10 \\
657.85 \\
21139.26 \\
3379.55 \\
138677.30 \\
205207.95\end{array}$ & $\begin{array}{r}\text { Adj SS } \\
620.58 \\
32267.79 \\
4829.51 \\
3636.10 \\
657.85 \\
21139.26 \\
3379.55 \\
138677.30\end{array}$ & $\begin{array}{r}\text { Adj MS } \\
206.86 \\
2933.44 \\
439.05 \\
110.18 \\
19.93 \\
174.70 \\
9.31 \\
30.09\end{array}$ & $\begin{array}{r}F \\
6.87 \\
97.47 \\
14.59 \\
3.66 \\
0.66 \\
5.81 \\
0.31\end{array}$ & $\begin{array}{r}P \\
0.000 \\
0.000 \\
0.000 \\
0.000 \\
0.929 \\
0.000 \\
1.000\end{array}$ \\
\hline
\end{tabular}

From this analysis, it can be seen that most of the interactions between the parameters are significant. The more interesting results are non-significant interactions. These are the interactions of R-Class with $\alpha, \mathrm{R}$-Class with $\gamma$, R-Class with $\alpha$ and $\gamma$, and R-Class with $\beta$ and $\alpha$. This result is interesting in terms of some of the data presented in this chapter. In the earlier figures, the data for the R-Class and the individual samples were averaged over the other parameters. For example, for Figure 40, which shows the RClass and $\alpha$ results, the data shown are averaged across the other parameters of $\beta$ and $\gamma$. Figures 44 through 46 present individual interactions rather than the averaged data to 
illustrate the interaction of the variables.

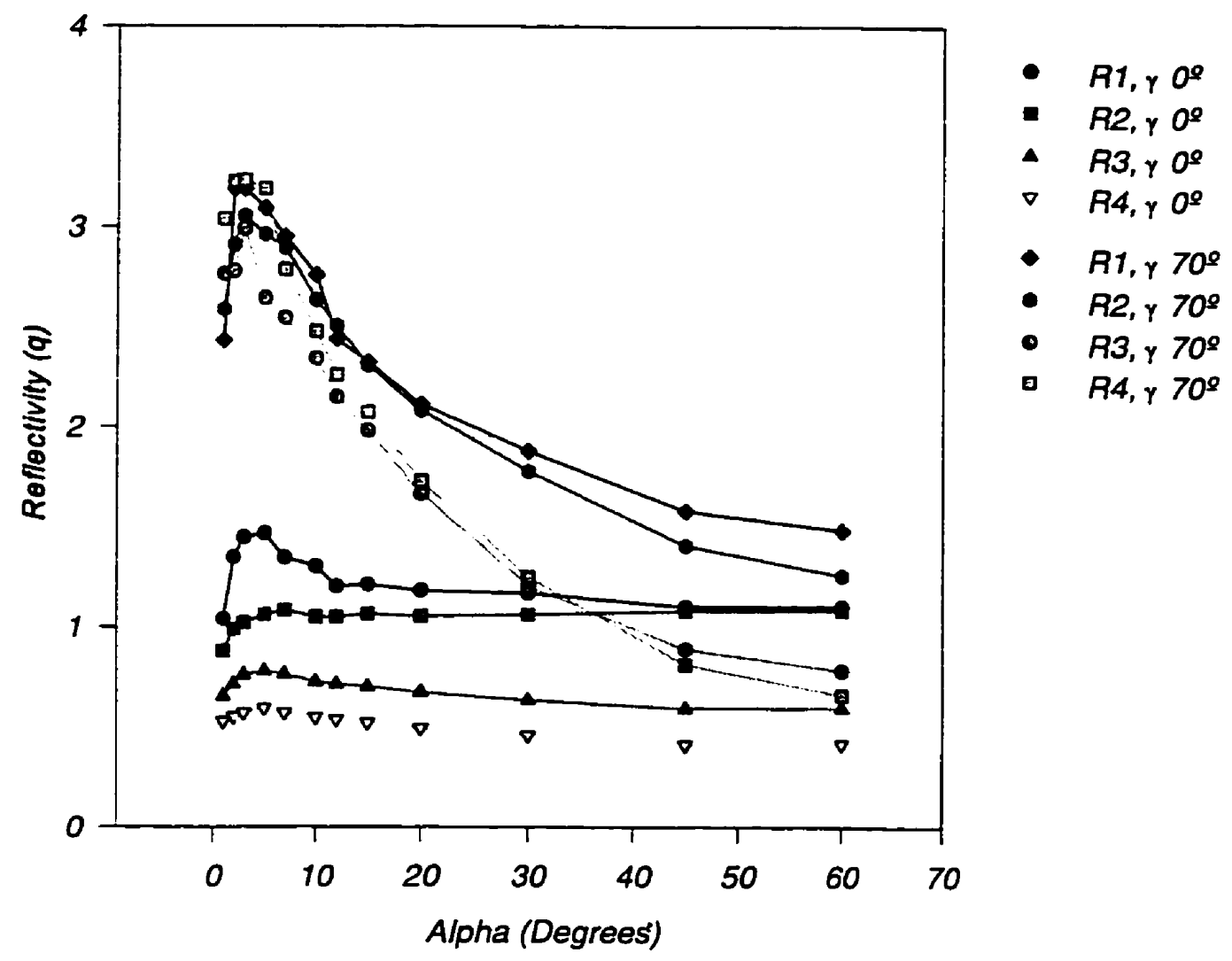

Figure 44 - Interaction of R-Class, $\gamma$ and $\alpha$ 


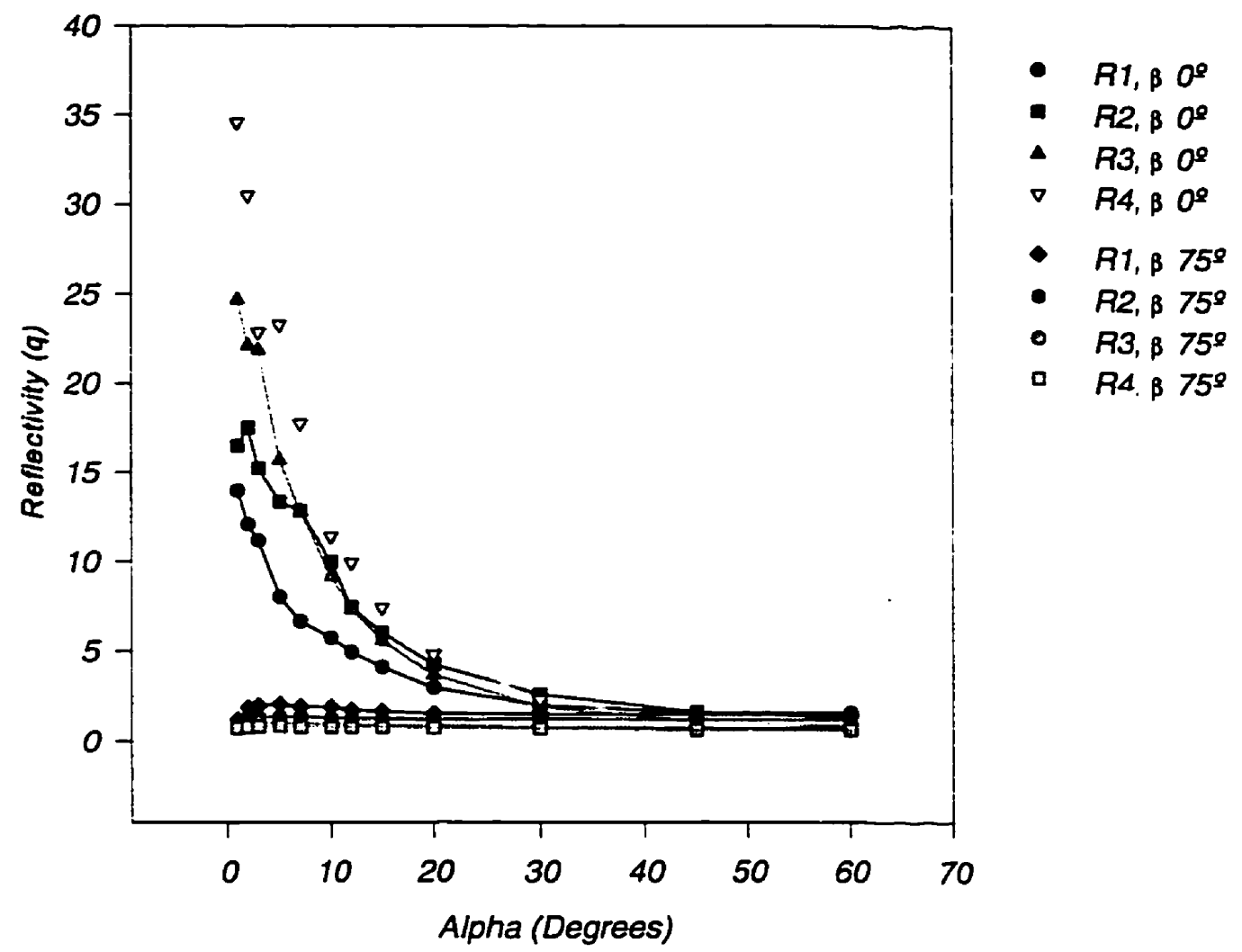

Figure 45 - Interaction of R-Class, $\alpha$, and $\beta$ 


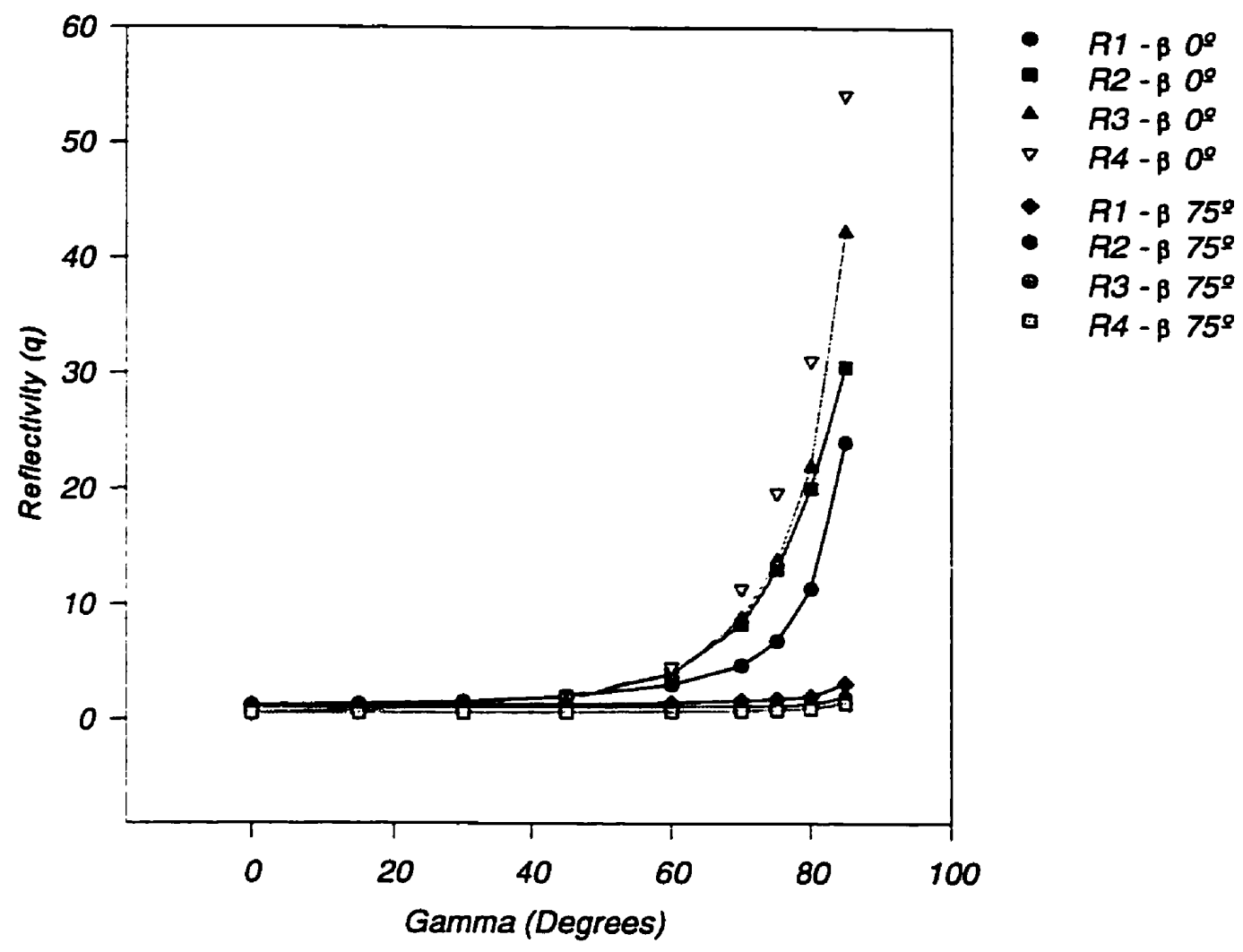

Figure 46 - Interaction of R-Class, $\beta$ and $\gamma$

In order to use the data for the calculation of the target luminance, the extent of the interaction in these experimental results requires extensive use of look-up tables similar in manner to the use of the standardized r-tables. To further establish the nature of the pavement reflection, these data were analyzed in terms of the pavement classification system results. This analysis is shown in section 5.5 .

\subsection{Influence of the Sample Rotation, $\delta$}

The final angle investigated was the rotational observation angle, $\delta$. It might be expected 
that, since the surface wear on the pavement is different along the roadway than across it, the reflection profile would be different with the angle of rotation. As discussed earlier, the influence of this angle was investigated by fixing the observation and incident angles and rotating the sample, Saskatchewan \#10, through $360^{\circ}$ measuring at every $15^{\circ}$. The measurements were performed for a single observation angle, $\alpha=5^{\circ}$, and two incidence angles, $\gamma=0^{\circ}$ and $\gamma=60^{\circ}$. The result at each $\delta$ was compared to the average of the results for the same $\gamma$. The results of this measurement series are shown in Figure 47 .

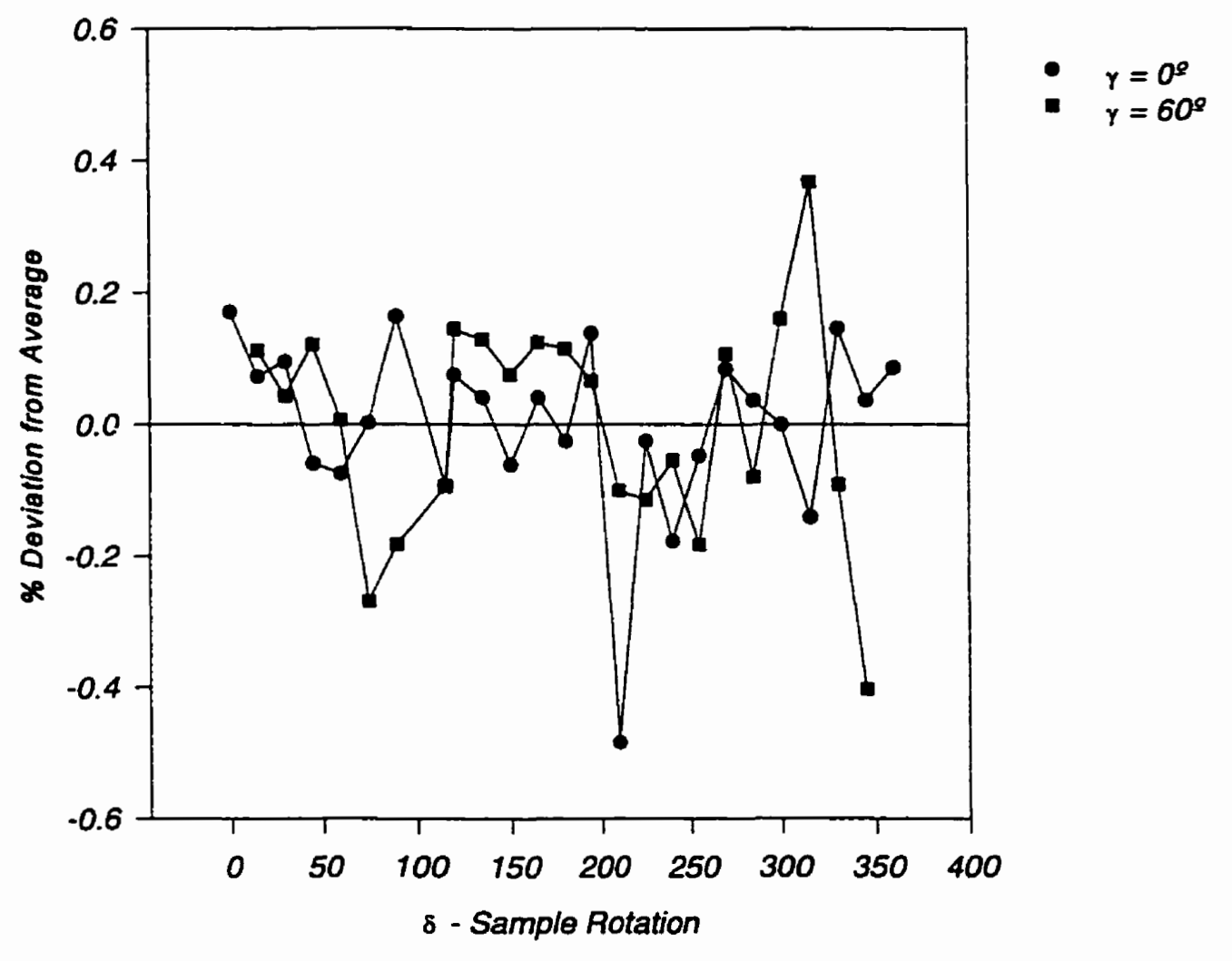

Figure 47 - Ratio of Measured Luminance to Average Measurement for Varying $\delta$ 
An ANOVA was performed on these results to determine if $\delta$ is a significant parameter in the reflection profile. These results are shown in Table 17. The ANOVA table shows that $\delta$ does not have a significant effect on the reflection profile.

Table 17 - ANOVA Table for $\delta$ Influence

\begin{tabular}{|c|c|c|c|c|c|c|}
\hline Analysi & Var & nce for & io Measur & Lumir & ance $t$ & - Average \\
\hline $\begin{array}{l}\text { Source } \\
\text { Delta } \\
\text { Gamma } \\
\text { Error } \\
\text { Total }\end{array}$ & $\begin{array}{r}\text { DF } \\
22 \\
1 \\
22 \\
45\end{array}$ & $\begin{array}{r}\text { SS } \\
0.51626 \\
0.00000 \\
0.51161 \\
1.02787\end{array}$ & $\begin{array}{r}\text { MS } \\
0.02347 \\
0.00000 \\
0.02326\end{array}$ & $\begin{array}{r}F \\
1.01 \\
0.00\end{array}$ & $\begin{array}{r}P \\
0.492 \\
1.000\end{array}$ & \\
\hline
\end{tabular}

This result is the same as those of CIE Technical Commitee 4-25. The influence of $\delta$ was neglected for the rest of the reflection analysis. However, the $\delta$ angie will be used in the calculation of the indirect illuminance on the target as specified in Equation 13 as this is required to complete the cosine relationship of the direct intensity.

\subsection{Pavement Classification System Comparisons}

In the reflection coefficient data presented earlier in section 5.1, as $\alpha$ was increased, the data trended towards a horizontal line. This was also evident in the $\beta$ and the $\gamma$ data. This effect implies a trend in the reflection profile towards a diffuse character. As the reflection profile of a sample changes, the characteristics which have been used to describe the sample change. The pavement classification systems are all based on $\alpha=1^{\circ}$ reflection data. With the data for a variable observation angle, the criteria such as $\mathrm{q}_{0}$ can 
now be related to observation angle. This means that for the angles chosen in this experiment, each sample has values for each classification parameter. The change in the criteria of the various classification systems was investigated.

\subsubsection{CIE System}

In the CIE system, $q_{0}$ represents the overall reflection of the incident light through a solid angle. In the data showing the influence of the observation angle, $\alpha$, on the reflection coefficient, the average of $q$ rose to a maximum at $\alpha=3^{\circ}$ or $5^{\circ}$ and then fell continuously with the increase in $\alpha$. It is expected that $\mathrm{q}_{0}$ would follow the same trend. This can be seen in Figure 48. Road class $\mathbf{R} 2$ typically shows a higher overall reflectance since it contains the high reflectance of the concrete samples Manitoba PR 200, Quebec \#4, and Quebec \#6.

As the reflection profile becomes more diffuse, as seen in the reflection data, the first specular factor, $\mathrm{S} 1$ is expected to decrease to a constant value with an increased observation angle. This trend is seen in Figure 49. Finally, the secondary specular factor, $S 2$, is the relationship of $q_{0}$ to the reflectance of a source vertically over the sample. It would be expected that as the reflection profile became more diffuse, $q_{0}$ would become closer in value to the $q(0,0)$. This would mean that $\$ 2$ would decrease with an increased observation angle. This trend is seen in Figure 50. 


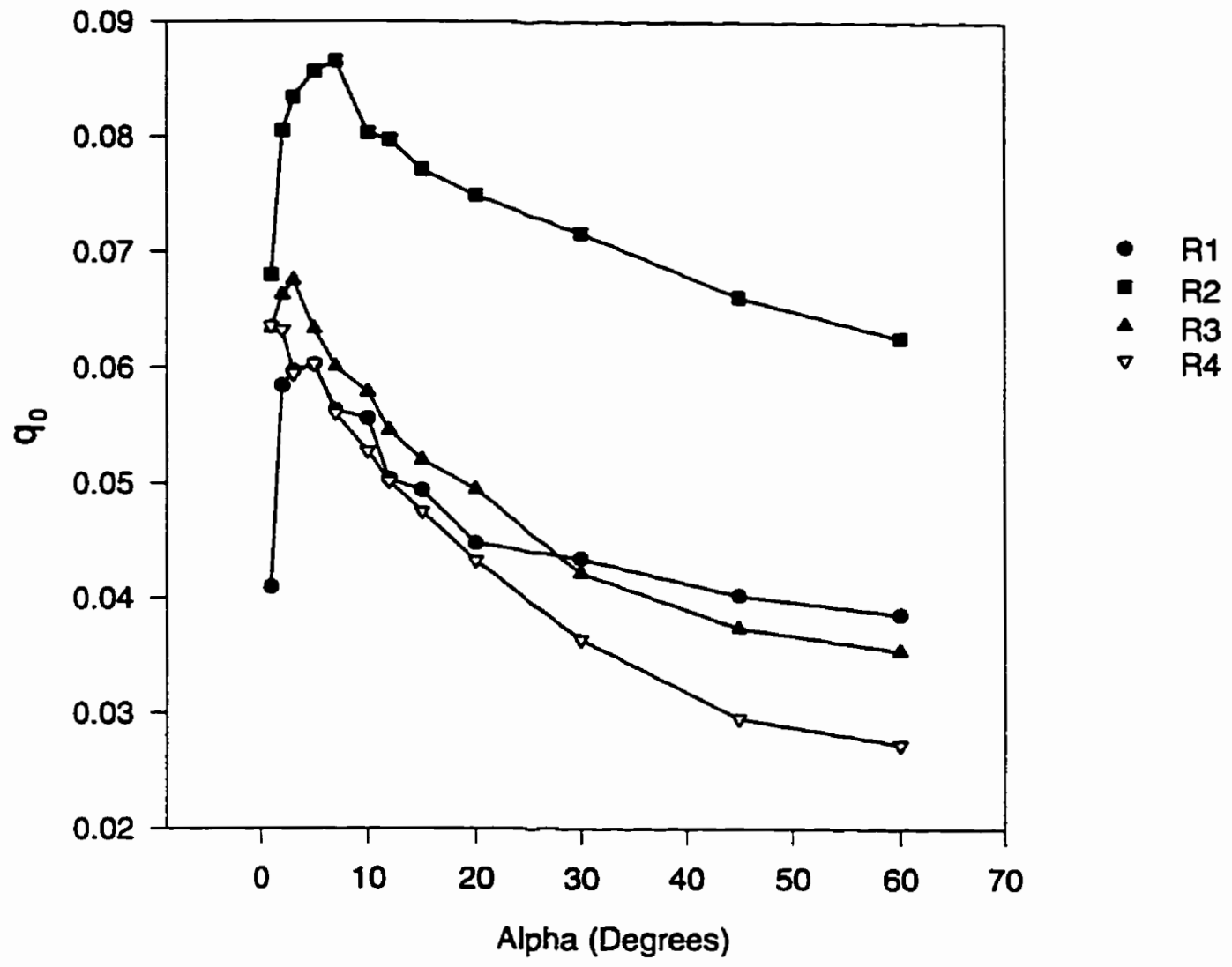

Figure 48 - Change in $\mathrm{q}_{0}$ with Observation Angle, $\alpha$, for All R-Classes 


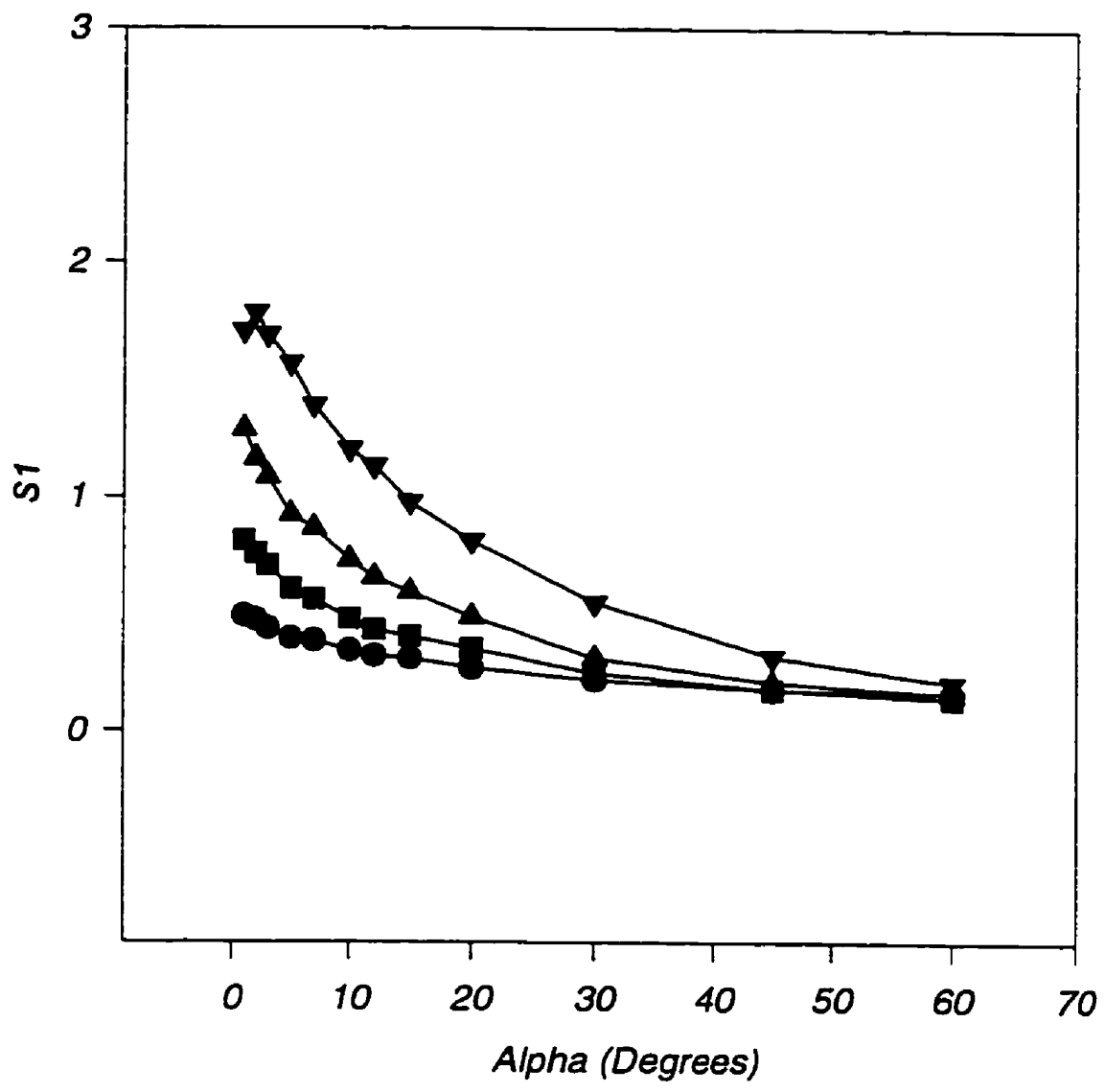

\begin{tabular}{ll}
- & $R 1$ \\
$\square$ & $R 2$ \\
\hline & $R 3$ \\
$\nabla$ & $R 4$
\end{tabular}

Figure 49 - Change in S1 with Observation Angle for all R-Classes 


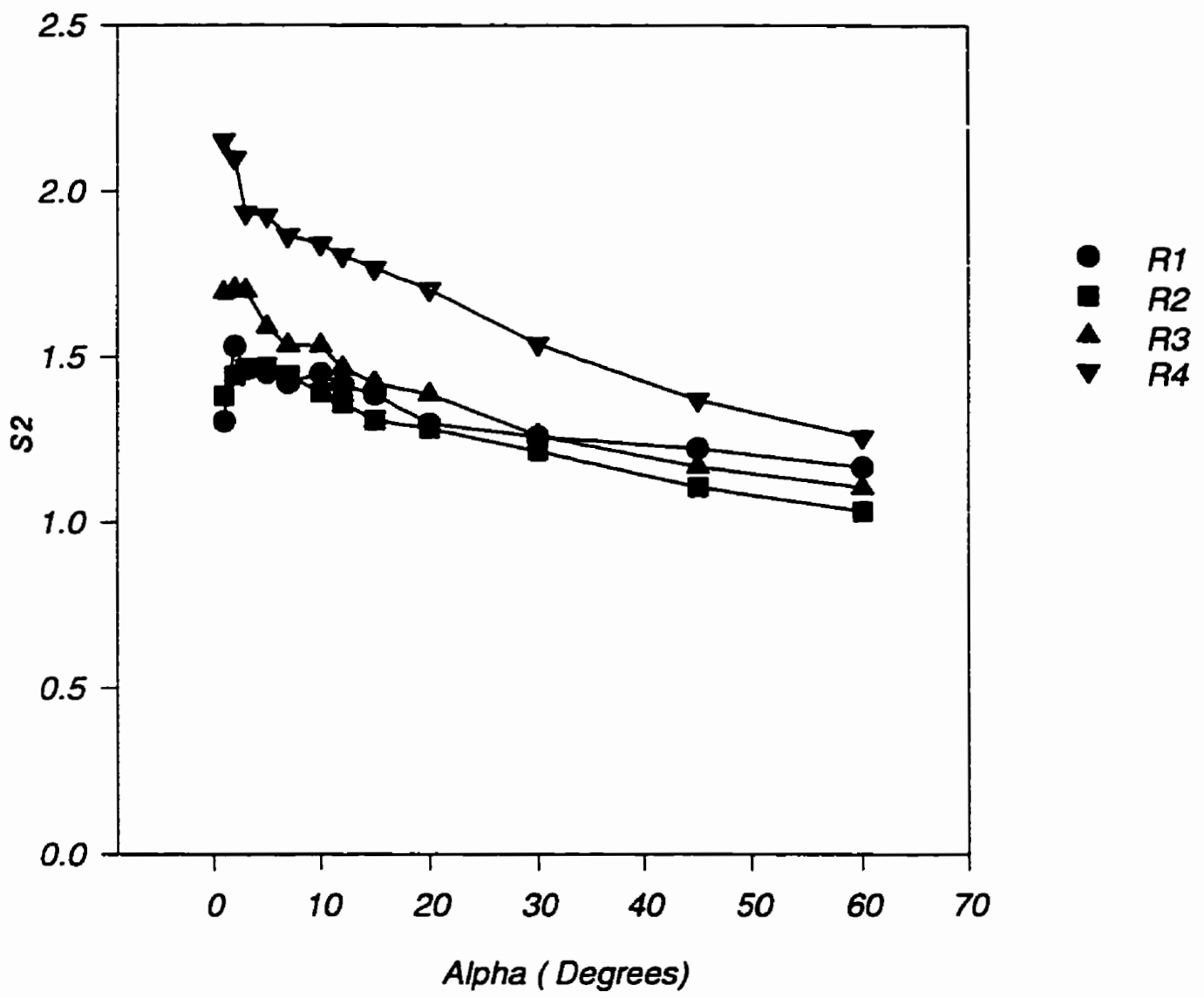

Figure 50 - Change in S2 with Observation Angle, $\alpha$

From the diagrams, it can be seen that the CIE system performs as expected with increased observation angle. The significant aspect of this data is the relationship of the $q_{0}$ parameter to the observation angle. As discussed earlier, the $q_{0}$ parameter represents the lightness of the pavement. The relationship of $\mathrm{q}_{0}$ to $\alpha$ shows that the pavement lightness changes with the observation angle. Typically, in the luminance calculation the pavement reflection data is scaled by the ratio of the pavement $q_{0}$ to the standard r-table $\mathrm{q}_{0}$, accounting for variations in pavement lightness. The results of the change in 
observation angle data show that a single $\mathrm{q}_{0}$ scaling factor cannot be used in the target luminance calculation.

\subsection{2 $Q_{d}$ System}

The calculation of $Q_{d}$ is very similar to that of $q_{0}$. According to Equations 27 and 31 , the true difference between $Q_{d}$ and $q_{0}$ is the $\cos (\gamma)$ factor in the numerator and the denominator of the $Q_{d}$ calculation. Like $q_{0}, Q_{d}$ has a dependency on $\alpha$. This dependency is shown in Figure 51 . Like $\mathrm{q}_{0}, \mathrm{Q}_{\mathrm{d}}$ rises to a maximum and then falls continuously as $\alpha$ is increased further. For this experiment, $Q_{d}$ and $q_{0}$ can be compared. These parameters are compared in Figure 52, which shows $\mathrm{Q}_{\mathrm{d}}$ and $\mathrm{q}_{0}$ for all samples at all $\alpha$ angles. 


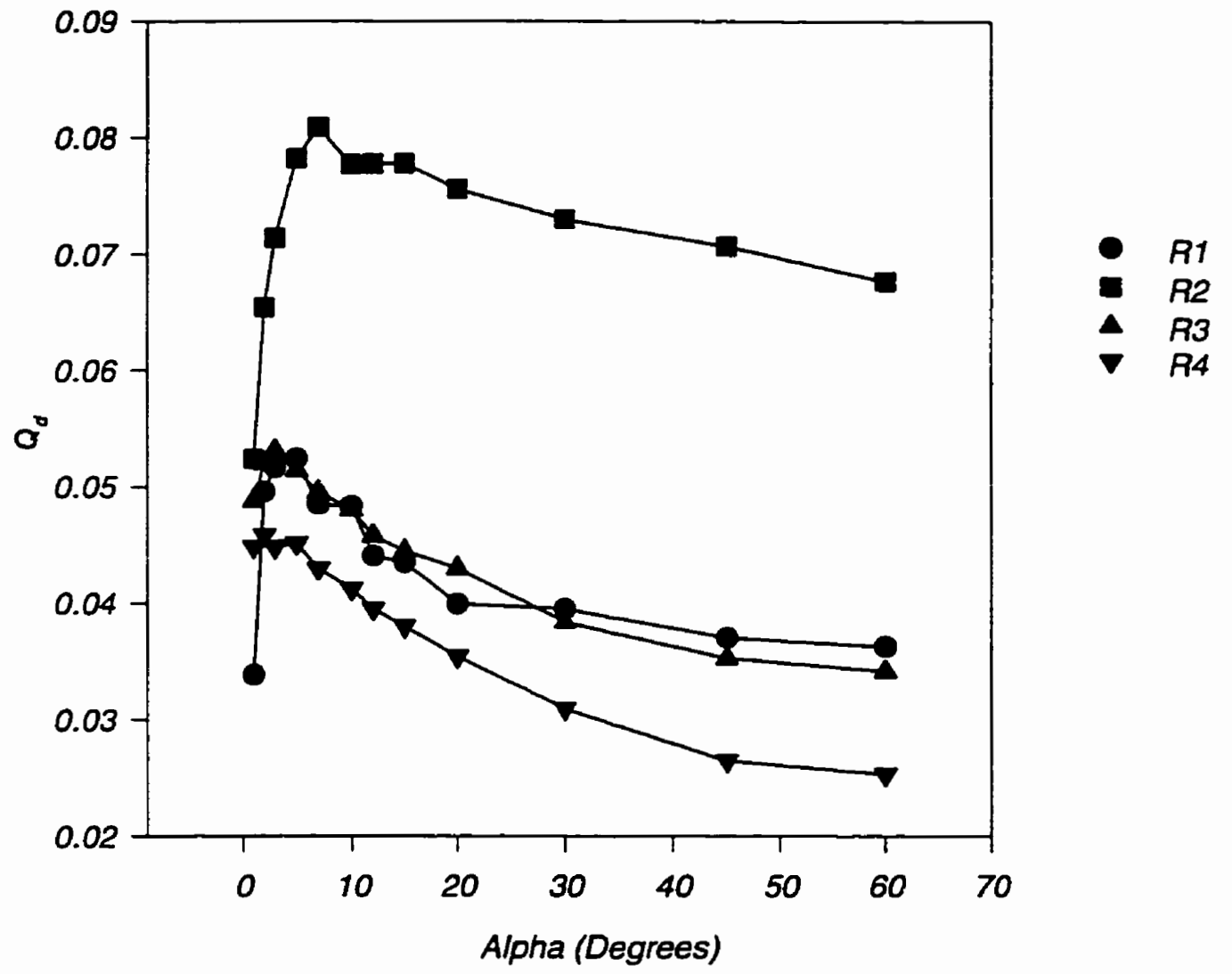

Figure 51 - Influence of Observation Angle on $Q_{d}$ 


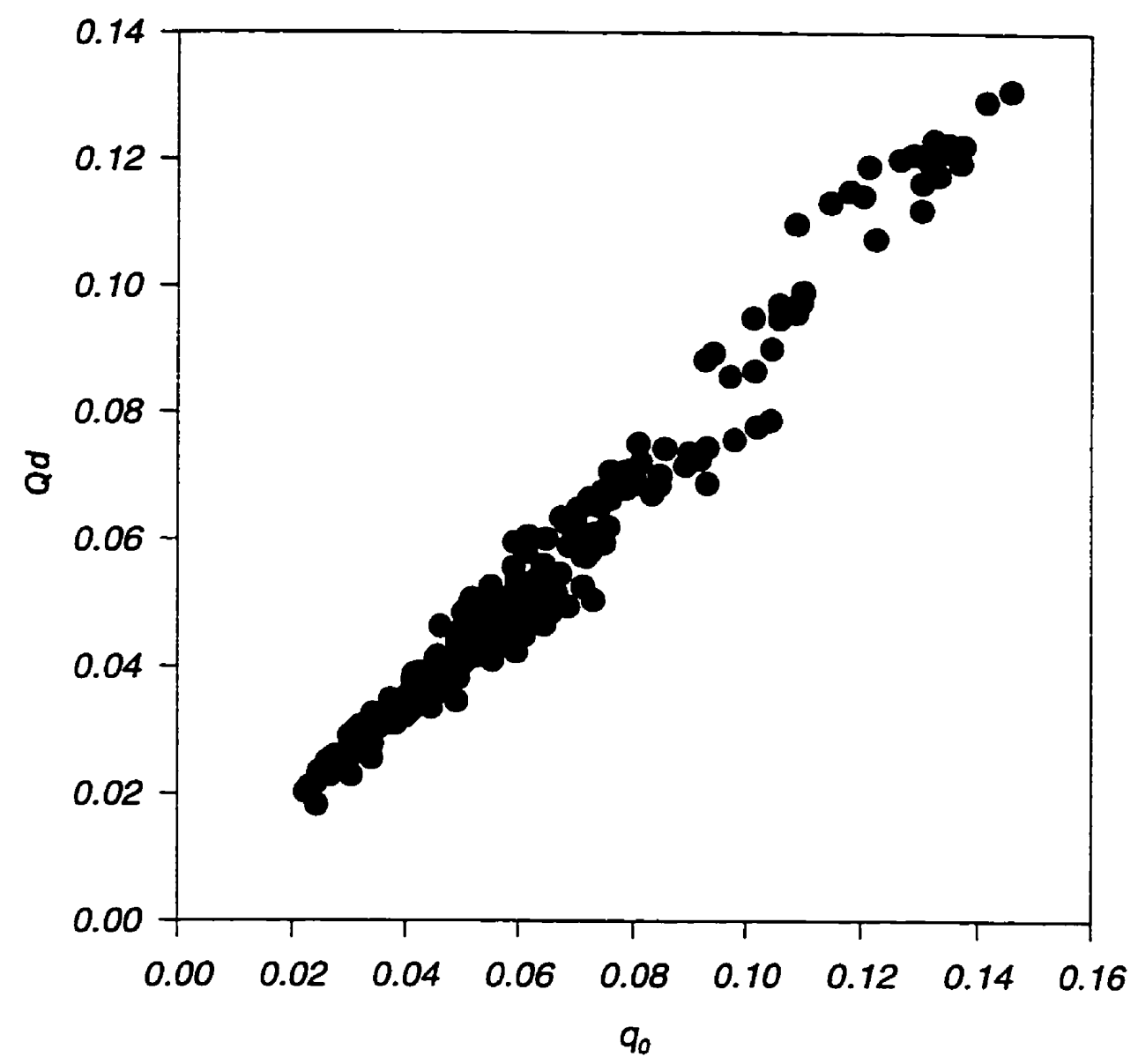

Figure 52 - Correlation of $q_{0}$ to $Q_{d}$

A linear regression was performed with the $\mathrm{q}_{0}$ and $\mathrm{Q}_{d}$ data. The result of the regressions is shown in Equation 39.

$$
q_{0}=1.07 * Q_{d}+0.00472
$$

The $r^{2}$ correlation coefficient between the $q_{0}$ and $Q_{d}$ parameters is .972. Brusque and 
Carta [1997] investigated the relationship of these two parameters based on $\alpha=1^{\circ}$ investigations of French pavement surfaces. In their investigations, it was found, as in this investigation, that $q_{0}$ and $Q_{d}$ are linearly related.

These results show that there is no significant difference in the two parameters. The usage of either parameter makes relatively no difference in the classification and description of the pavement surfaces. The choice of parameters should be made based on the lighting application. As explained earlier, for in situ measurements, the parameter $Q_{d}$ is easily measured using an already designed device but there is some question of the reliability of the $Q_{d}$ factor as a multiplier in the pavement luminance parameter. Further work has been performed by Brusque and Carta to investigate the applicability of the $Q_{d}$ parameter to designed and actual roadway lighting installations. They found that the use of the $\mathrm{q}_{0}$ more accurately allows the calculation of the pavement luminance in the resulting lighting installation and recommend the continued use of the $q_{0}$ parameter.

In this research, $q_{0}$ will be used in the analysis of the reflection data.

\subsubsection{K System}

The $x$ system, although replaced by the existing CIE system, has never been analyzed in terms of the influence of the observation angle. This analysis was performed for both the $\kappa$ and $\kappa_{p}$ parameters. These results are shown in Figures 53 and 54. The data presented in these figures is averaged across each of the road classes. The discontinuity in road 
classes $R 1$ and $R 3$ was a result of some of the measured samples having exceedingly high $\mathrm{K}$ values. The difficulty in using the $\mathrm{K}$ parameter for the observation angle is found in the use of the minimum $r$ value for the calculation which can occur at any $\gamma$ point on the $\beta=0^{\circ}$ plane. For many samples, the minimum occurred at $\gamma=0^{\circ}$. As the observation angle increases, the reflection profile becomes more diffuse and the minimum can occur at many different locations. The discontinuity is not seen when the $\kappa_{p}$ parameter is used in the classification system. 


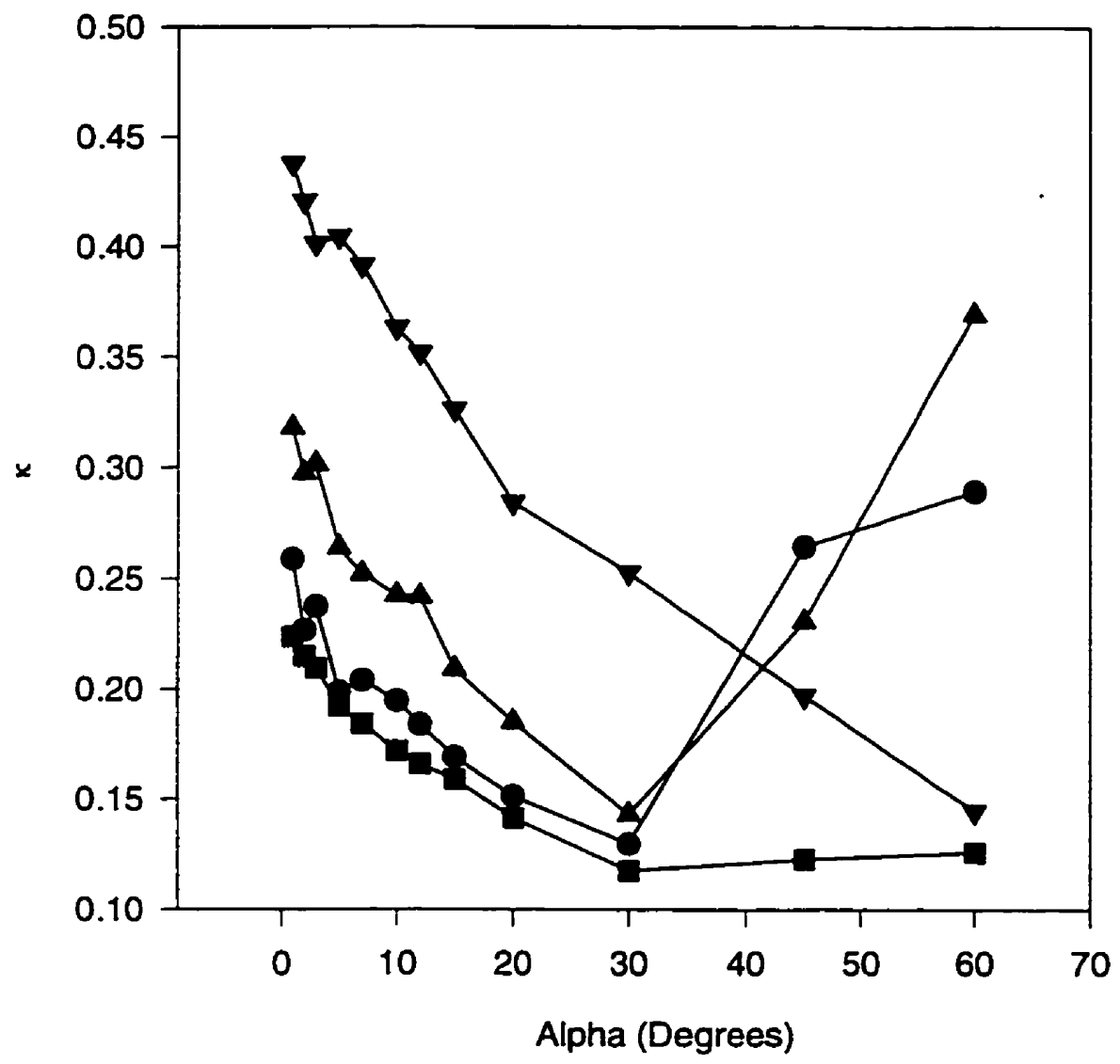

- R1

- $\mathrm{R} 2$

$\Delta \mathrm{R3}$

$\nabla$ R4

Figure 53 - Influence of Observation Angle, $\alpha$, on $\kappa$ 


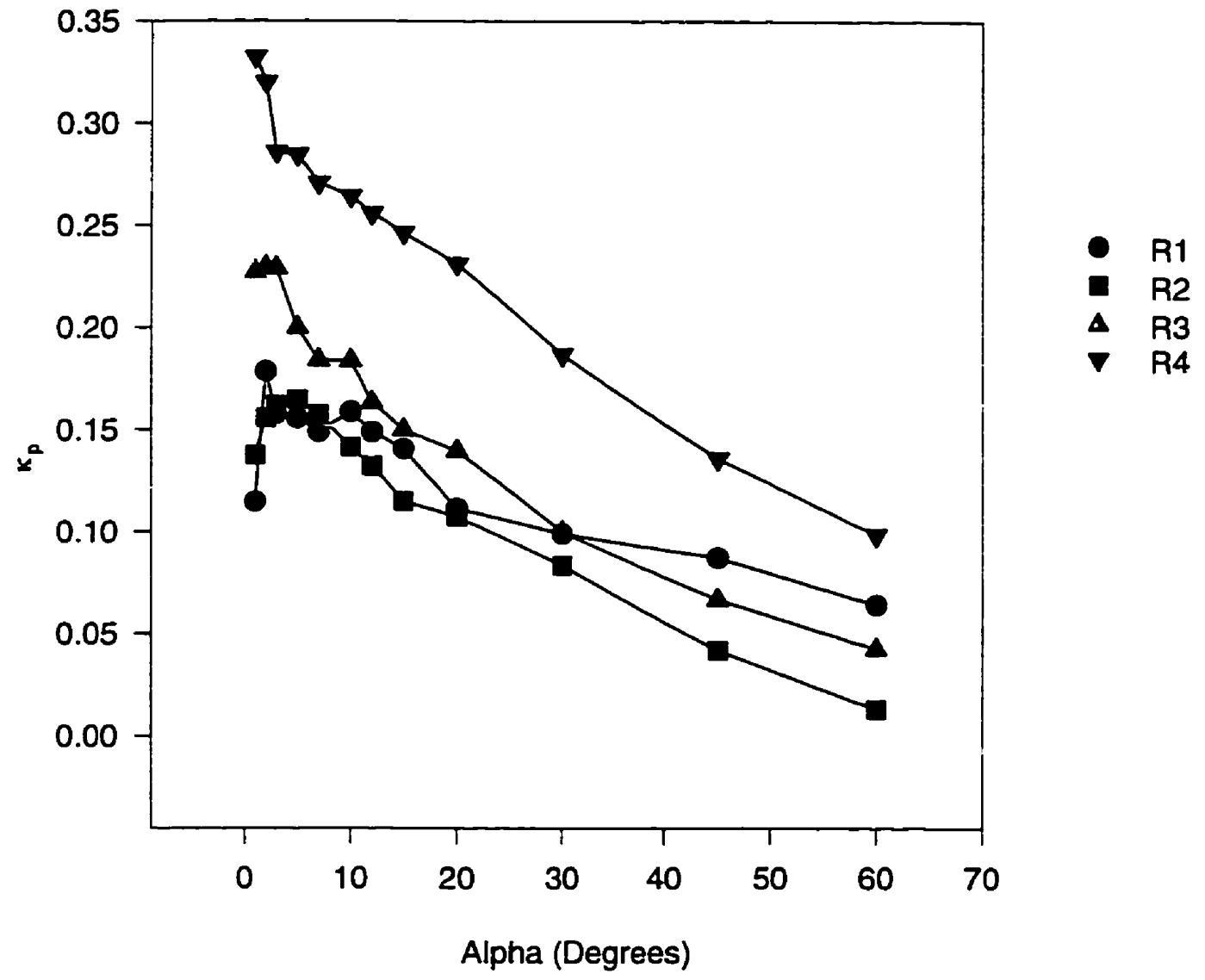

Figure 54 - Influence of Observation Angle, $\alpha$ on $\kappa_{p}$

The $\kappa_{\mathrm{p}}$ definition is very similar to the CIE parameter $\mathrm{S} 2$ which replaced the $\mathrm{k}$ parameter in the classification system. According to Equations 29 and 33, there should be a log relationship between these two parameters.

Since the $\kappa$ parameter is not continuous for all samples across the observation angle and the information in the $\mathrm{k}_{\mathrm{p}}$ parameter is also found in the $\$ 2$ parameter, these parameters will not be considered in any further analysis. 


\subsubsection{Alcade System}

As discussed previously, the Alcade system was designed to account for the macro and micro roughness in the sample. The parameter $A_{D}$, was established to account for specular reflection. Parameters $A_{11}$ and $A_{12}$, were each established to account for the diffuse reflection. The $A_{12}$ was designed to account for the diffuse nature of the reflection profile across the sample and $A_{11}$ was to account for the diffuse nature in the direction of travel. The change in the Alcade parameters with observation angle is presented in Figures 55 through 57 . As expected, the specular parameter $A_{D}$, decreases with increased observation angle. The other parameters, $A_{11}$ and $A_{12}$, were calculated with $r(0,0.625)$ in the denominator. As the reflection profile becomes more diffuse, it also becomes more spherical. This means that the difference between the reflection value of any two points on the reflection profile becomes smaller. This is seen in both of the $A_{11}$ and $A_{12}$ parameters. The length of the spectrum shortens and becomes wider as $A_{11}$ decreases and $A_{12}$ increases. Both of these parameters, particularly $A_{11}$, come close to being constant at very high observation angles. 


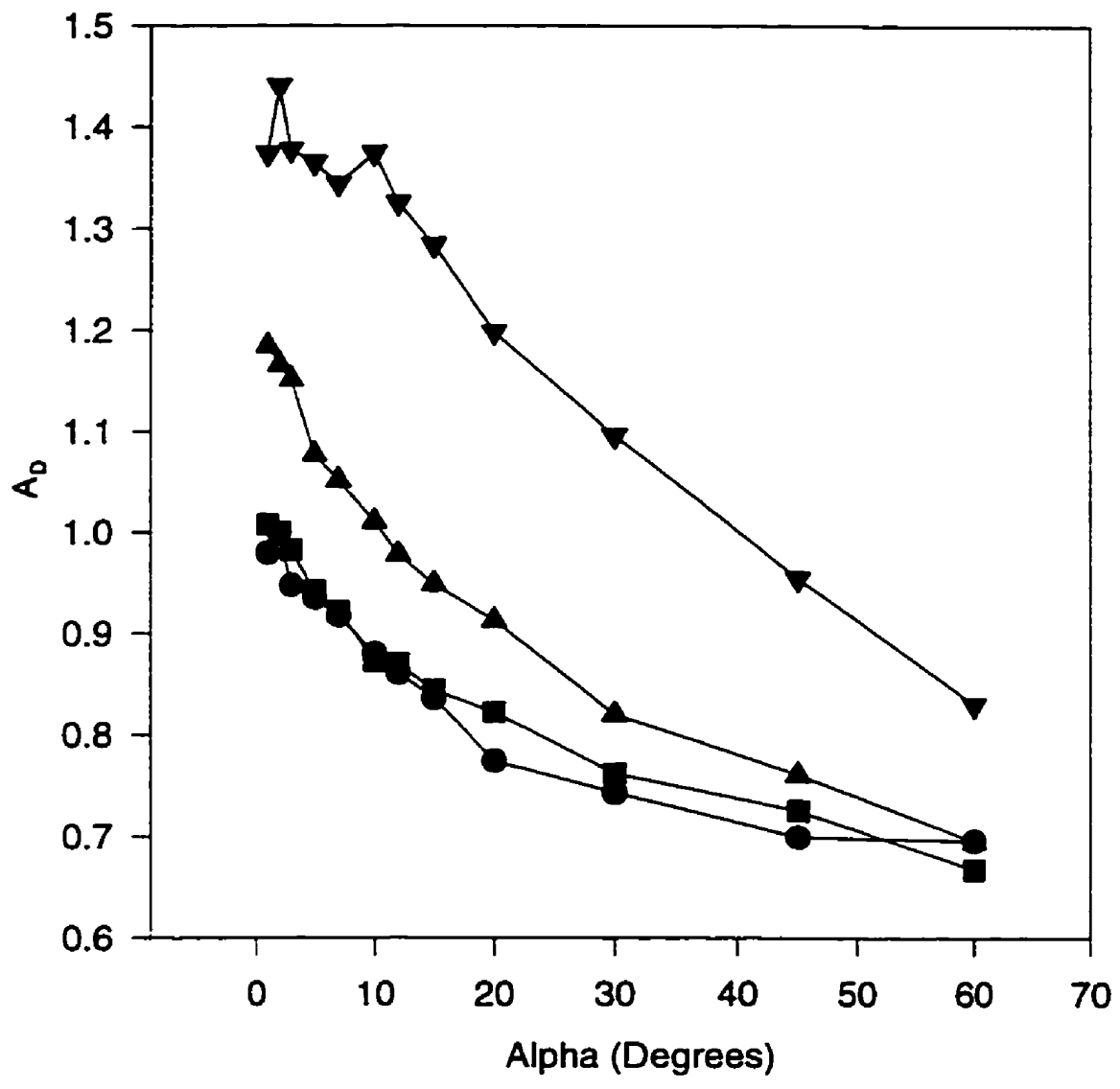

$\begin{array}{ll}- & R 1 \\ \nabla & R 2 \\ \nabla & R 3 \\ \nabla & R 4\end{array}$

Figure 55 - Influence of Observation Angle, $\alpha$, on $A_{D}$ 


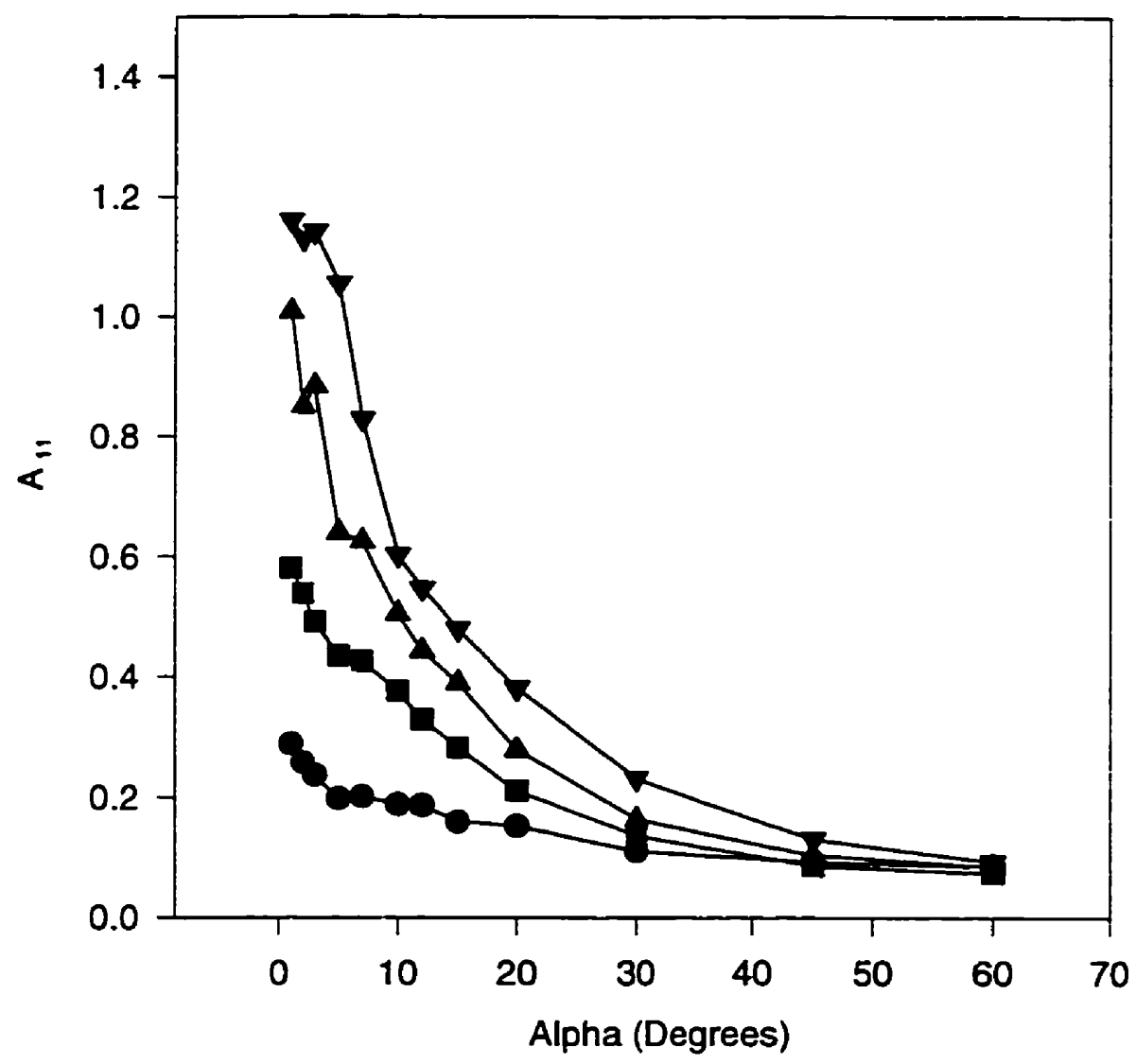

- R1

- $\mathrm{R2}$

- $\mathrm{R3}$

$\nabla \mathrm{R4}$

Figure 56 - Influence of Observation Angle, $\alpha$, on the $A_{11}$ Parameter 


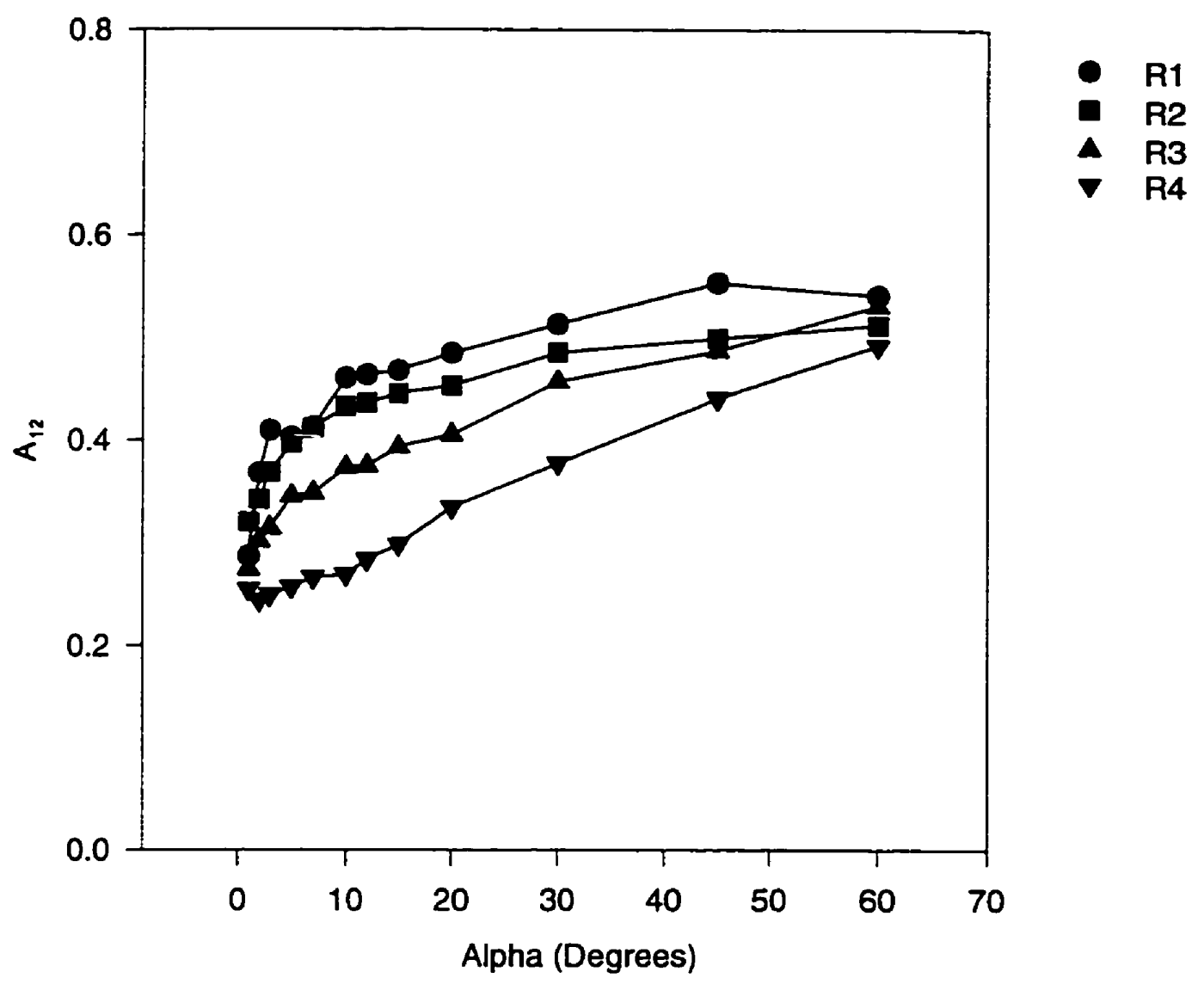

Figure 57 - Influence of Observation Angle, $\alpha$, on parameter $A_{12}$

It is noteworthy that the Alcade system does not include a parameter which is related to the overall brightness, like $\mathrm{a} \mathrm{q}_{0}$ parameter. It is unknown at this time how the Alcade system would be used in lighting calculations since the overall brightness cannot be accounted in the calculation.

The relationship of the Alcade parameters to the pavement roughness will be investigated in a surface roughness experiment, discussed later. Based on this, the 
applicability of the Alcade model as compared to the CIE model can be established.

\subsubsection{Correlation of Classification Systems}

The correlation of the CIE system and that of Alcade was investigated by establishing Pearson correlation coefficients between the various factors. For this correlation, the calculated parameters at each $\alpha$ angle for each sample was used. The results of this correlation are shown in Table 18.

Table 18 - Correlation of CIE and Alcade Classification Parameters

\begin{tabular}{|lrrrcc|}
\hline & 00 & $S 1$ & $S 2$ & $A D$ & A11 \\
S1 & -0.029 & & & & \\
S2 & -0.038 & 0.854 & & & \\
AD & -0.233 & 0.878 & 0.814 & & \\
A11 & 0.137 & 0.889 & 0.739 & 0.613 & \\
A12 & 0.267 & -0.795 & -0.620 & -0.916 & -0.570 \\
\hline
\end{tabular}

It is evident in these results, that all of the parameters are reasonably correlated except for the $q_{0}$ parameter which does not have a matching parameter in the Alcade system. The correlation between $S 1, S 2, A_{D}$ and $A_{11}$ was also expected since the calculation for these factors is similar. The negative correlation of $\mathrm{S} 1$ to the $\mathrm{A}_{12}$ parameter is more interesting. The $A_{12}$ parameter is based on a factor which is calculated at $\beta=90^{\circ}$ rather than at $\beta=0^{\circ}$. Since $S 1$ represents the length of the reflection profile and $A_{12}$ represents the width, this correlation relationship shows that as the reflection profile becomes longer or more specular, the reflection to the side or across the roadway diminishes. This relationship is shown in Figure 58. 


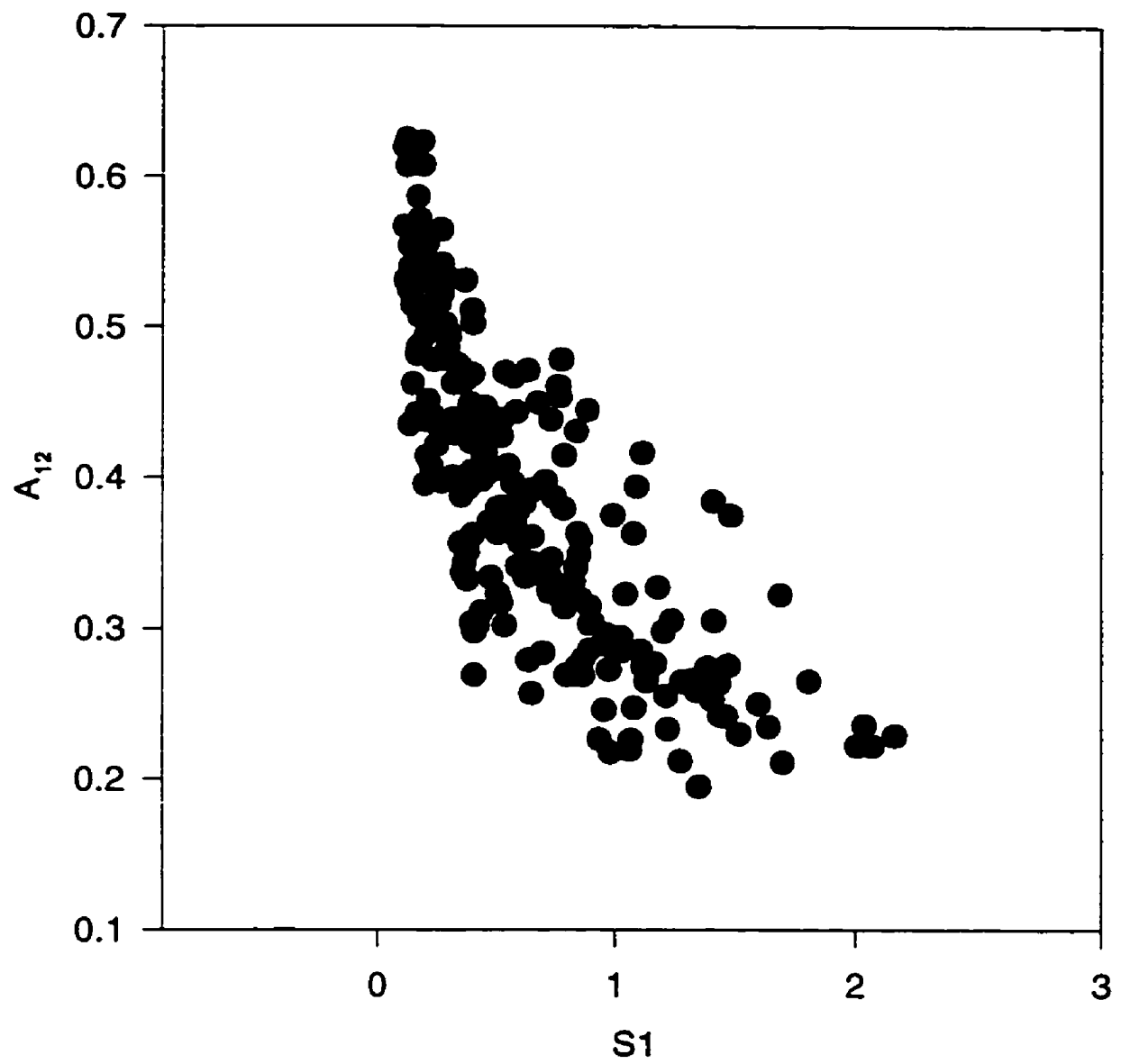

Figure 58 - Correlation of $\mathrm{S} 1$ and $\mathrm{A}_{12}$

\subsection{Conclusion}

The reflection profile for varying angles of observation and incidence was investigated for 20 pavement samples ranging from highly specular to highly diffuse reflection modes. The interaction of the various angular measurements was also investigated. It was found that the reflection profile is dependant on all the angles of $\alpha, \beta$, and $\gamma$. The 
reflection profile was also found to be dependent on the sample type and the R-Class pavement classifications. It was found that the reflection profile is not dependent on the sample rotational angle, $\delta$. Due to the extent of the interactions in the data, further investigations into a mathematical model are required.

The classification parameters of the CIE, the proposed $Q_{d}$ parameter of TC 4-25, the older $\mathrm{K}$ system, and the proposed classification system of Alcade were also investigated in terms of the change in observation angle. It was found that the proposed $Q_{d}$ system is closely related to that of the existing $q_{0}$ system and further application studies are required to investigate the applicability of this parameter. These investigations are outside of the scope of this research. Similarly, the former system of $\kappa$ is not stable at high observation angles and, in any case, the $\kappa_{\mathrm{p}}$ parameter is related to the specular factor S2. Based on these results, this older system does not provide any further insight into the nature of the reflection profile. The existing system and the Alcade were both found to be consistent with the change in observation angle results. It was also found that the CIE system and the Alcade system are reasonably closely correlated.

An investigation into the surface roughness character of samples is required to further establish the relationship of the sample to the reflection profile which is discussed in Chapter 6. 


\section{Surface Roughness Experiment}

In order to investigate the relationship of the surface roughness to the reflection character of the pavement, a second experiment which measured the surface roughness was undertaken. Since the pavement reflection properties are dependent on the texture and the structure of the surface, the mean roughness and the correlation distance of the surface was measured. The relationship between these values and the pavement classification indices of the CIE and the Alcade classification systems was established.

\subsection{Experimental Design}

The sample surface roughness was established based on the standard deviation of the change in surface height measured across the sample. These measurements were taken on a pavement sample using a pantograph measurement system. The height of the surface was measured at every millimeter across the middle of the sample both with and across the direction of travel. The standard deviation of the surface height measurements was then used to represent the mean surface roughness.

However, the mean roughness of the sample surface is not a sufficient descriptor of the character of a surface. Two surfaces might have an equal mean roughness but two very different characters. The other characteristic which must be added to the roughness description is the correlation distance. As discussed earlier, the surfaces are made up of several stones and facets. The correlation distance represents the size of the facet. This relationship is shown in Figure 59. 


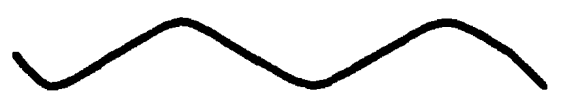

a)

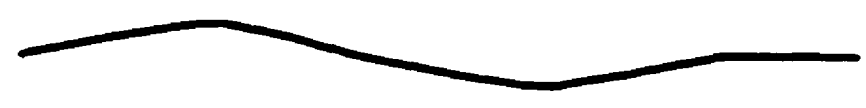

b)

Figure 59 - Surface Height and Autocorrelation Distance

Figure 59 shows two surfaces with the same surface height deviation but different autocorrelation distances.

The nature of these surfaces is such that they can be described as a random rough surface. This means that there is no mathematical explanation for the changes in surface height and the processes which describe the surface roughness are entirely random. This is true for pavement surfaces, but like a time series, a distance series has an autocorrelation aspect. For autocorrelation to exist, a data point must depend on the point before it. This relationship is shown in Equation 40.

$$
\epsilon_{t}=\rho \epsilon_{t-1}+u_{t}
$$

$\epsilon_{\mathrm{t}}$ is the data point at distance $t . \mu_{1}$ is the disturbance function for the distance $t$ and $\rho$ is the autocorrelation factor, $|\rho|<1$. At the next data point, $\epsilon_{t+1}$ is dependent on the previous point and the point before. This relationship leads to the definition that a data point is dependent on a linear combination of the data points before it as shown in Equation 41 . 


$$
\epsilon_{t}=\sum_{t=0}^{\infty} \rho^{t}
$$

The autocorrelation factor between two data points is a linear combination of the points before them. As the distance, $t$, grows greater, the relationship between the starting point and the ending point becomes less and less represented by the function $\rho^{\prime}$. An autocorrelation function close to one represents a high correlation and an autocorrelation function close to zero represents little correlation.

The correlation distance for the rough surfaces was defined by Beckmann and Spizzichino [1963]. They proposed that the correlation distance was defined by the point where the autocorrelation function is equal to $e^{-1}$. It should be noted that the autocorrelation function is not related to the mean roughness of the surface. It might be expected that a surface which has a small mean roughness would have a large correlation distance since the surface is very flat. This is not necessarily true, and the roughness although small, might have very short facet length's that change directions very quickly.

\subsection{Surface Roughness Measurement}

The roughness of the pavement surfaces was measured using a pantograph and a stylus system. The pantograph set the position of the stylus relative to the pavement surface, and the stylus traces the surface roughness. A potentiometer was mounted on the fixed rod. The stylus was attached to the rotating control arm of the potentiometer. As the stylus moved across the surface, the potentiometer control would rotate, changing the 
resistance of the potentiometer. The measurements were made by recording the resistance. The system schematic is shown in Figure 60.

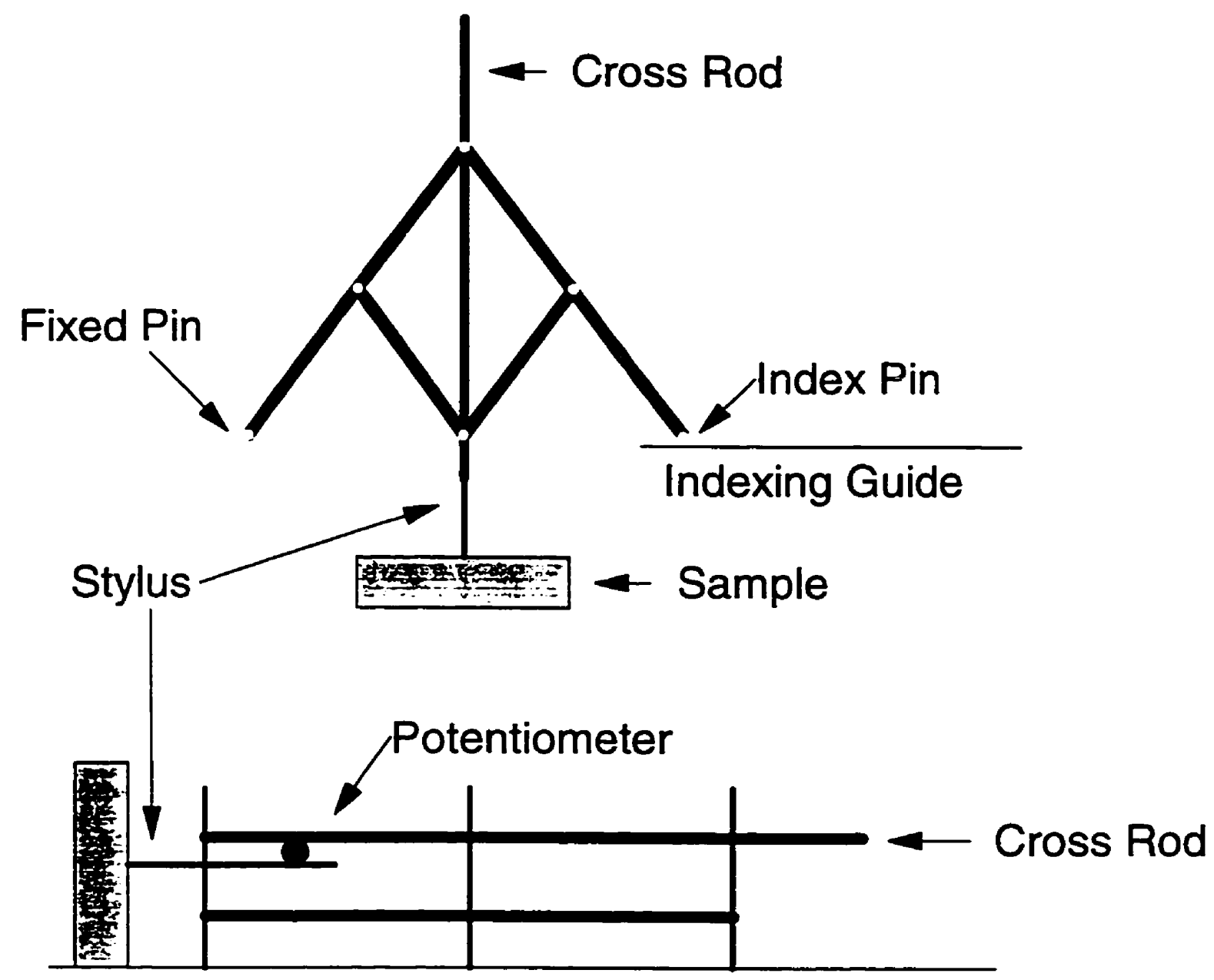

Figure 60 - Plan and Profile Schematic of Surface Roughness Pantograph

The resistance system was calibrated using a surface of known roughness and recording the resistance measurement. The relationship of the distance to the resistance measured is a linear relationship as shown in Figure 61. 


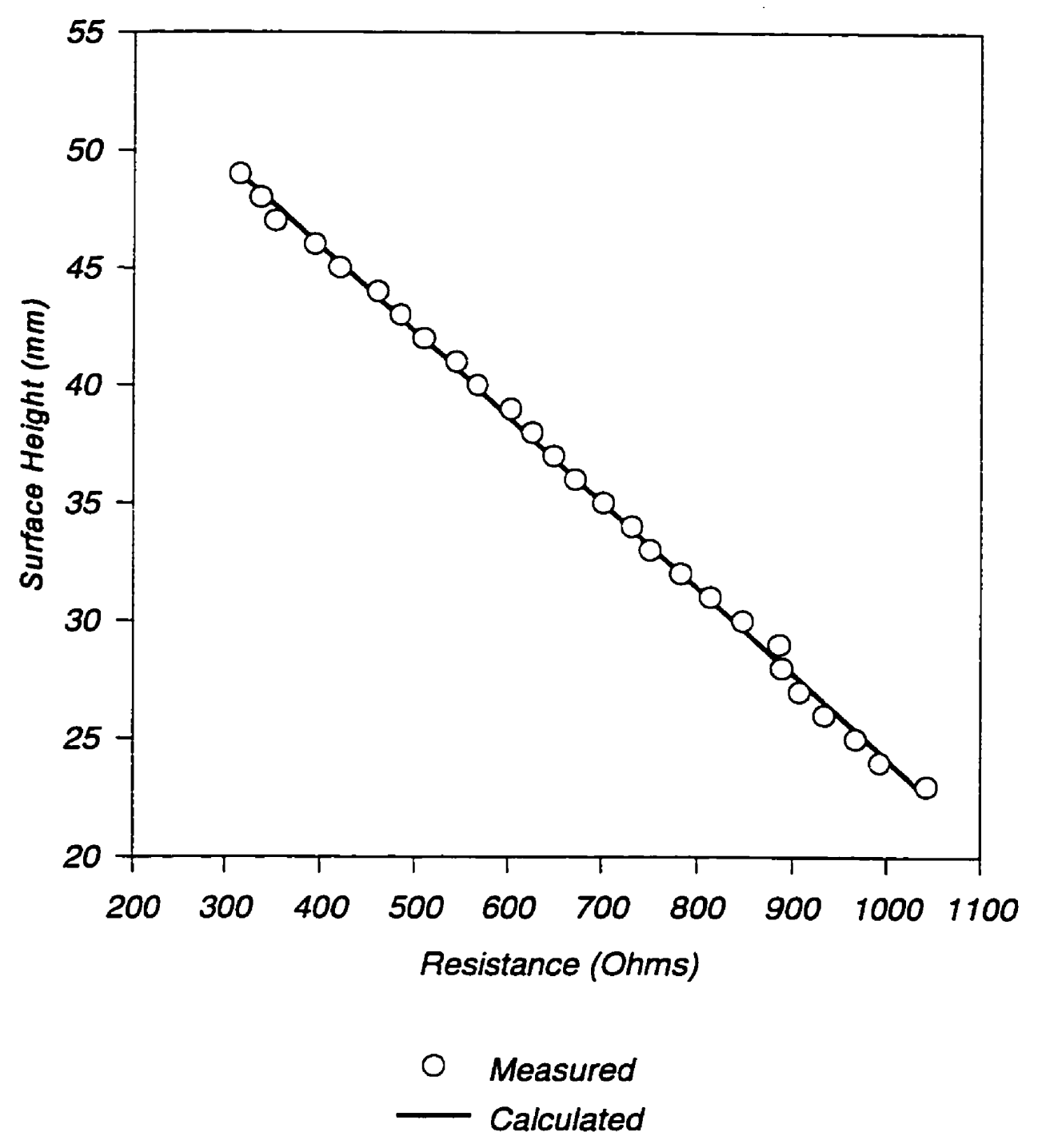

Figure 61 - Calibration of the Pantograph

This measurement system is only capable of measuring the macro-roughness of the surface. Micro-roughness is invisible to the eye and also undetectable by this system. 


\subsection{Experimental Uncertainty}

The uncertainty for the surface roughness measurement was estimated in the same way as the reflection data. Sample Saskatchewan \#10 was measured five times. The average roughness and the correlation distance was calculated for each set of measurements. Again, the uncertainty was calculated as the standard deviation of the mean. This resulted in a $4.4 \%$ percent uncertainty of the average roughness and a $12.1 \%$ uncertainty of the correlation distance.

For this uncertainty calculation, the surface roughness was measured at the centre of the sample. To measure a more representative portion of the pavement, the sample was measured at several locations across the surface both with and across the direction of travel. In these measurements, the roughness was estimated from the average from these measurements. The error of the average was also estimated from this series of test runs. The error of the mean for this group of measurements increased to $20.8 \%$ for the roughness and $21.7 \%$ for the correlation distance. These increased uncertainty values were used for the entire experiment.

\subsection{Results}

The measurement of the surface roughness for each pavement sample was performed. Figure 62 shows the change in surface height for sample Saskatchewan \#10. The mean roughnesses for each of the samples is shown in Table 19. 


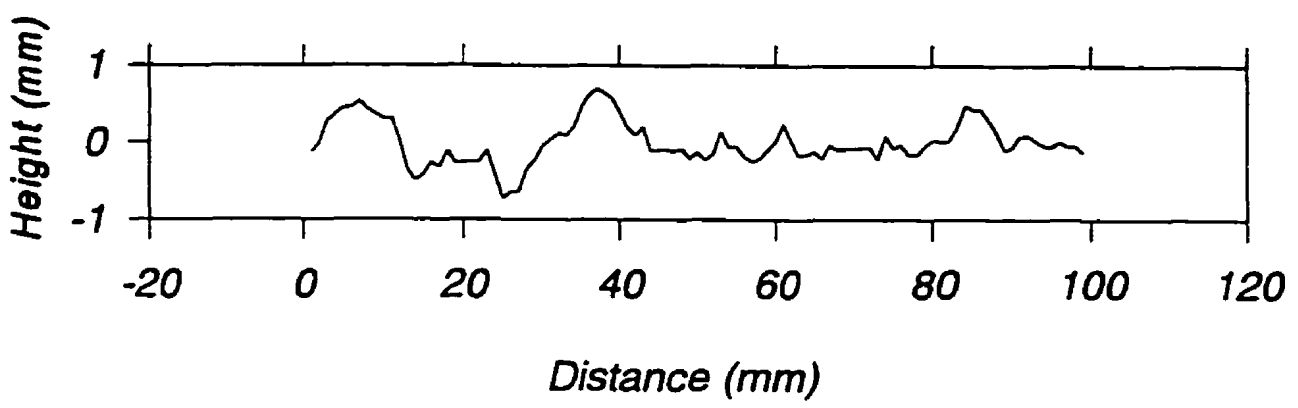

Figure 62 - Roughness Profile for Saskatchewan \# 10

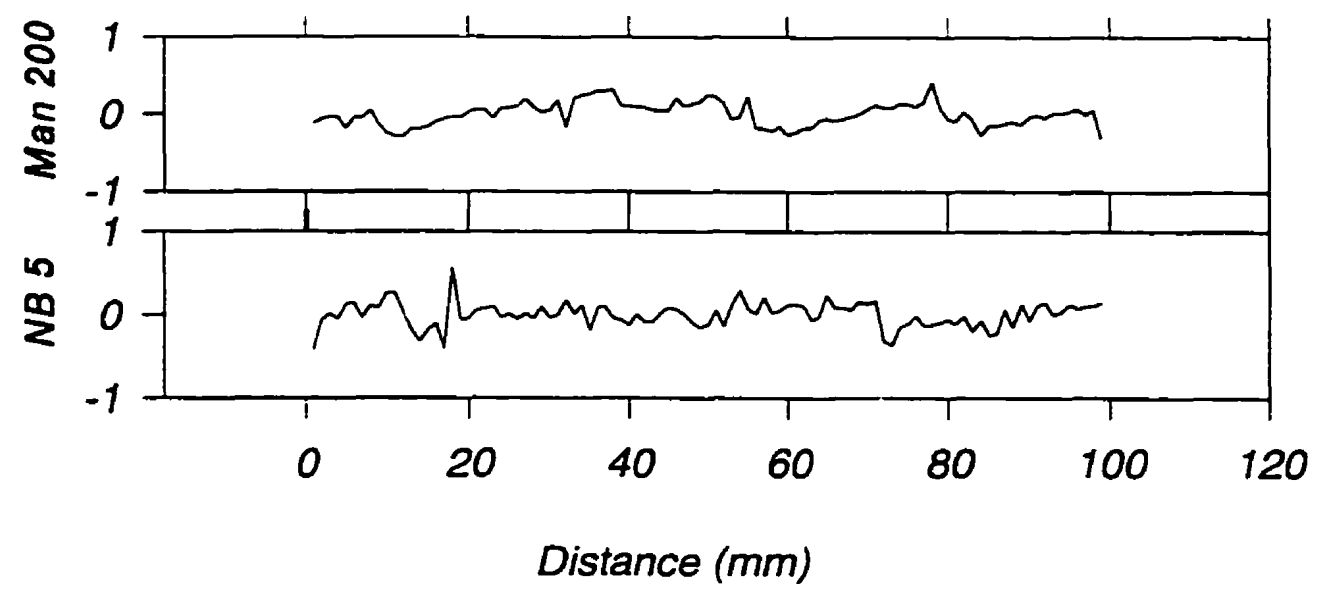

Figure 63 - Comparison of Roughness Profile for Manitoba 200 and New Brunswick 5

Figure 63 shows the autocorrelation relationship by comparing the roughness of New Brunswick 5 and Manitoba 200. Both of these curves have an equal roughness but have very different characteristics of autocorrelation. Manitoba 200 has long surfaces while 
New Brunswick \#5 has a short choppy surface characteristic. The autocorrelation distances calculated for all of the pavement samples are shown in Table 19.

Table 19 also shows the average of the across and with traffic results for the correlation distance and the roughness. The other items represented in these results are the area of the surface facets and the angle of the surface facets. The area is calculated as the across traffic correlation distance multiplied by the with traffic correlation distance. The facet angle is an estimation of the slope of facets. The facet angle is defined in Equation 42 and shown in Figure 64.

$$
\text { Facet Angle }=\sin ^{-1} \frac{\text { Surface Height }}{\text { Correlation Distance }}
$$

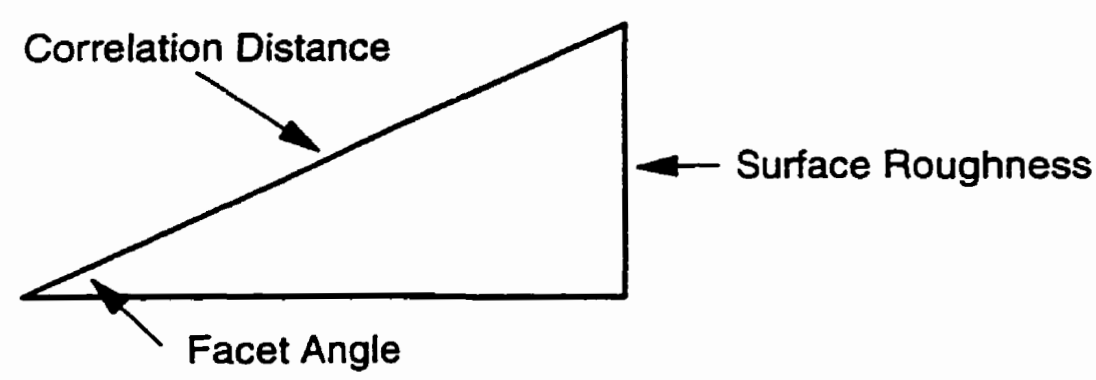

Figure 64 - Calculation of the Facet Angle 
Table 19 - Results of the Surface Roughness Experiment

\begin{tabular}{||l|l|l|l|l|l|l|l|l||}
\hline Sample Name & $\begin{array}{l}\text { Rough } \\
\text { With } \\
\text { Traffic }\end{array}$ & $\begin{array}{l}\text { Rough } \\
\text { Acr. } \\
\text { Traffic }\end{array}$ & $\begin{array}{l}\text { CD } \\
\text { With } \\
\text { Traffic }\end{array}$ & $\begin{array}{l}\text { CD } \\
\text { Acr. } \\
\text { Traffic }\end{array}$ & $\begin{array}{l}\text { Ave } \\
\text { Rough }\end{array}$ & $\begin{array}{l}\text { Ave } \\
\text { Corr } \\
\text { Dist }\end{array}$ & $\begin{array}{l}\text { Facet } \\
\text { Area }\end{array}$ & $\begin{array}{l}\text { Facet } \\
\text { Angle }\end{array}$ \\
\hline \hline Alberta \#1 & 0.799 & 0.937 & 3.697 & 2.518 & 0.868 & 3.107 & 9.308 & 16.221 \\
\hline British Columbia N4 & 0.138 & 0.196 & 1.888 & 3.459 & 0.167 & 2.674 & 6.532 & 3.576 \\
\hline Manitoba PR 200 1BWP & 0.339 & 0.153 & 0.705 & 4.628 & 0.246 & 2.667 & 3.264 & 5.297 \\
\hline New Brunswick R12 \#5 & 0.179 & 0.153 & 1.277 & 0.79 & 0.166 & 1.034 & 1.009 & 9.247 \\
\hline Nova Scotia \#11 & 0.116 & 0.241 & 0.722 & 0.919 & 0.178 & 0.821 & 0.664 & 12.559 \\
\hline Nova Scotia \#15 & 0.872 & 0.871 & 2.604 & 2.085 & 0.872 & 2.344 & 5.427 & 21.832 \\
\hline Ontario 19-14 & 0.162 & 0.256 & 2.189 & 1.912 & 0.209 & 2.051 & 4.187 & 5.855 \\
\hline Ontario 19-17 & 0.179 & 0.393 & 1.505 & 0.623 & 0.286 & 1.064 & 0.938 & 15.595 \\
\hline Ontario 19-3 & 0.157 & 0.136 & 1.836 & 3.305 & 0.147 & 2.57 & 6.067 & 3.275 \\
\hline Ontario 19-7 & 0.167 & 0.251 & 1.843 & 1.59 & 0.209 & 1.716 & 2.93 & 6.997 \\
\hline Ontario L7-2 & 0.522 & 0.619 & 2.869 & 2.133 & 0.571 & 2.501 & 6.12 & 13.19 \\
\hline Ontario L7-3 & 0.348 & 0.62 & 1.938 & 3.237 & 0.484 & 2.587 & 6.272 & 10.783 \\
\hline Ontario L7-4 & 0.409 & 0.329 & 1.98 & 1.57 & 0.369 & 1.775 & 3.107 & 11.992 \\
\hline PEI \#2-K & 0.21 & 0.307 & 2.368 & 1.955 & 0.258 & 2.161 & 4.629 & 6.86 \\
\hline Quebec \#4 & 0.165 & 0.15 & 8.635 & 1.761 & 0.157 & 5.198 & 15.201 & 1.736 \\
\hline Quebec \#6 & 0.239 & 0.231 & 5.133 & 8.35 & 0.235 & 6.741 & 42.856 & 1.996 \\
\hline Saskatchewan WL\#10 & 0.281 & 0.174 & 3.915 & 3.012 & 0.228 & 3.463 & 11.792 & 3.774 \\
\hline Saskatchewan WL \#32 & 0.59 & 0.522 & 3.895 & 2.857 & 0.556 & 3.376 & 11.126 & 9.479 \\
\hline Saskatchewan WL \#37 & 0.357 & 0.357 & 2.386 & 3.974 & 0.357 & 3.18 & 9.484 & 6.446 \\
\hline Saskatchewan WL \#8 & 0.242 & 0.242 & 5.024 & 2.81 & 0.242 & 3.917 & 14.116 & 3.537 \\
\hline \hline
\end{tabular}

\subsection{Correlation to Surface Recipe}

As mentioned previously, for some of the samples, the material recipe was provided

through the earlier work of the University of Toronto. This information is presented in

Table 20. 
Table 20 - Material Composition of Samples

\begin{tabular}{|c|c|c|c|c|c|c|c|c|c|}
\hline Sample Name & $\begin{array}{l}\% \\
\mathrm{C} \\
\end{array}$ & $\begin{array}{l}\begin{array}{l}\text { Coarse } \\
\text { Material }\end{array} \\
\end{array}$ & $\begin{array}{l}\% \\
\mathbf{F}\end{array}$ & $\begin{array}{l}\text { Fine } \\
\text { Material } \\
\end{array}$ & $\begin{array}{l}\% \\
\text { B }\end{array}$ & Stb & F1 & AV & VMA \\
\hline Alberta \#1 & 60 & Sandstone & 40 & Natural Sand & 5.6 & 11.7 & 2.07 & 3.8 & 14.1 \\
\hline British Columbia N4 & 40 & $\begin{array}{l}\text { Granite / } \\
\text { Siltstone }\end{array}$ & 60 & Sand / Shale & 5.6 & & & & \\
\hline Manitoba PR 200 & 40 & $\begin{array}{l}\text { Limestone } \\
\text { Granite }\end{array}$ & 60 & Sand & & & & & \\
\hline New Brunswick \#5 & 25 & Quarry Rock & 75 & Sand & 6.5 & 13.9 & 3.2 & 3.88 & \\
\hline Nova Scotia \# 11 & 60 & Gray Wacke & 40 & Sand & 5.3 & 7.1 & 13 & 3 & 15.5 \\
\hline Nova Scotia \#15 & 55 & Gray Wacke & 45 & Sand & 5.3 & 6.4 & & 3.4 & 14.3 \\
\hline Ontario $19-14$ & 45 & Traprock & 55 & $\begin{array}{l}\text { Limestone / } \\
\text { Sand }\end{array}$ & 5.4 & 2.8 & 3.6 & 0.4 & 14.6 \\
\hline Ontario $19-17$ & 45 & Traprock & 55 & $\begin{array}{l}\text { Limestone / } \\
\text { Sand }\end{array}$ & 5.4 & 2.8 & 3.6 & 0.4 & 14.6 \\
\hline Ontario 19-3 & 45 & Traprock & 55 & $\begin{array}{l}\text { Limestone / } \\
\text { Sand }\end{array}$ & 5.4 & 2.8 & 3.6 & 0.4 & 14.6 \\
\hline Ontario 19-7 & 45 & Traprock & 55 & $\begin{array}{l}\text { Limestone / } \\
\text { Sand }\end{array}$ & 5.4 & 2.8 & 3.6 & 0.4 & 14.6 \\
\hline Ontario $47-2$ & 54 & Limestone & 45 & Sand & 4.8 & 12.5 & 3.9 & 2.3 & 13.7 \\
\hline Ontario $47-3$ & 54 & Limestone & 45 & Sand & 4.8 & 12.5 & 3.9 & 2.3 & 13.7 \\
\hline Ontario 17.4 & 54 & Limestone & 45 & Sand & 4.8 & 12.5 & 3.9 & 2.3 & 13.7 \\
\hline PEI \#2-K & 35 & Granite & 65 & Sand & 6 & 5.8 & 10.7 & 4.4 & \\
\hline Quebec \#4 & 40 & $\begin{array}{l}\text { Limestone / } \\
\text { Shale }\end{array}$ & 60 & Sand & & & & & \\
\hline Quebec \#6 & 40 & $\begin{array}{l}\text { Limestone / } \\
\text { Shale }\end{array}$ & 60 & Sand & & & & & \\
\hline Sask WL \# 10 & 40 & $\begin{array}{l}\text { Limestone } \\
\text { Granite }\end{array}$ & 60 & Quartzite & 6.1 & 2.96 & 3.2 & 1.1 & \\
\hline SaskWL \#32 & 65 & $\begin{array}{l}\text { Limestone } \\
\text { Granite }\end{array}$ & 35 & Sand & 5.3 & 3.6 & 4.2 & 5.6 & \\
\hline Sask WL \#37 & 65 & $\begin{array}{l}\text { Limestone } \\
\text { Granite }\end{array}$ & 35 & Sand & 5.3 & 3.6 & 4.2 & 5.6 & \\
\hline Sask WL \#8 & 40 & $\begin{array}{l}\text { Limestone } \\
\text { Granite }\end{array}$ & 60 & Quartzite & 6.1 & 2.96 & 3.2 & 1.1 & \\
\hline
\end{tabular}


The fields represented in Table 20 are as follows:

Table 21 - Fields in the Material Composition Table

\begin{tabular}{||l|l|}
\hline$\% C$ & Percent Coarse Aggregate \\
\hline Coarse Material & Coarse Aggregate Material \\
\hline$\%$ F & Percent Fine Aggregate \\
\hline Fine Material & Fine Aggregate Material \\
\hline$\% B$ & Percent Binder by Weight \\
\hline STB & Marshall Stability Test \\
\hline Fl & Marshall Flow Test \\
\hline AV & Air Voids in the Mixture \\
\hline VMA & Voids by MateriaLAggregate \\
\hline
\end{tabular}

Pearson correlation coefficients were established between this data and the surface roughness characteristics. For this correlation, all of the recorded roughness characteristics were used. The results of this calculation are shown in Table 22.

Table 22 - Correlation of Surface Roughness Characteristics to the Material Recipe

\begin{tabular}{|lrrrrrrr|}
\hline \multicolumn{7}{|l|}{ Correlations (Pearson) } \\
\multicolumn{70}{c|}{ \%C Agg } & \%F Agg & \% B & \multicolumn{1}{l|}{ Stab } & Flow & AV & VMA \\
RoughWT & 0.571 & -0.573 & -0.295 & 0.324 & -0.363 & 0.463 & -0.520 \\
RoughAT & 0.617 & -0.621 & -0.416 & 0.390 & -0.226 & 0.361 & -0.499 \\
CDWT & -0.078 & 0.082 & 0.233 & -0.216 & -0.407 & 0.096 & -0.627 \\
CDAT & -0.028 & 0.031 & -0.106 & -0.225 & -0.308 & 0.213 & -0.474 \\
Ave R. & 0.612 & -0.615 & -0.365 & 0.367 & -0.300 & 0.421 & -0.519 \\
Ave CD & -0.069 & 0.073 & 0.085 & -0.251 & -0.411 & 0.171 & -0.642 \\
Area & -0.090 & 0.094 & 0.175 & -0.278 & -0.371 & 0.181 & -0.581 \\
Angle & 0.494 & -0.499 & -0.387 & 0.447 & 0.067 & 0.243 & -0.178 \\
\hline
\end{tabular}

As expected, there is a comparatively high correlation between the roughness and the 
percent of coarse material and a negative correlation to the percent of fine aggregate. The correlation distance does not appear to be related to material recipe in any way. The only material factor which seems related consistently to the roughness parameters is the Voids by Material Aggregate but the data on this factor is not complete and no significant conclusion can be drawn on this variable. The relationship of the percent coarse aggregate to the roughness is shown in Figure 65. 


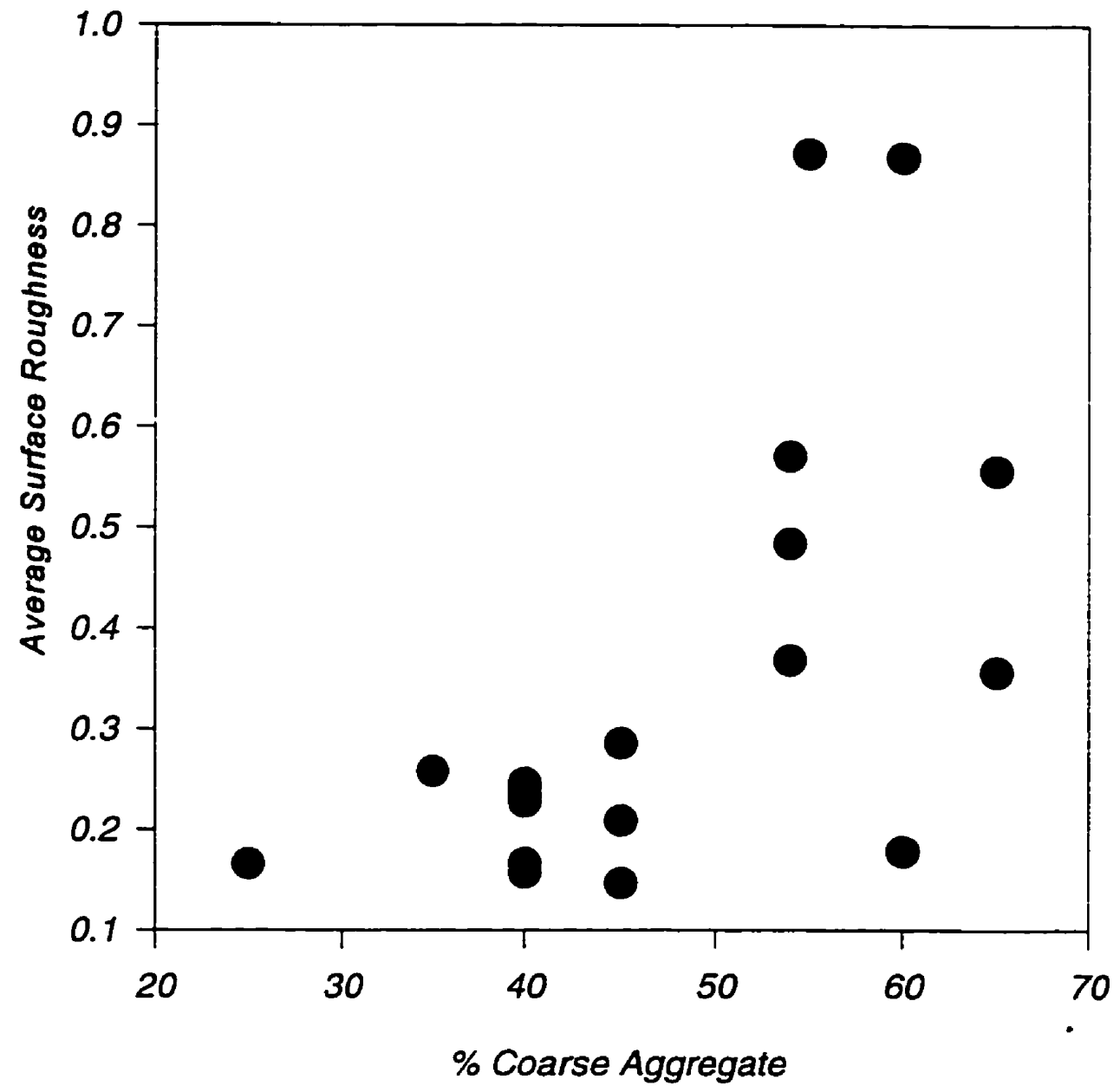

Figure 65 - Correlation of Percent Coarse Aggregate to Average Surface Roughness

The result in Figure 65 shows that there is a relationship of the pavement recipe to the roughness characteristics of the surface. This relation is not strong but can be used as a predictor of the roughness. The other aspects of the surface reflection character must be determined by the construction and the use of the roadway as well as the recipe. 


\subsection{Correlation to Surface Reflection}

Several different research projects have been undertaken to relate the surface roughness to the reflection characteristic of the sample. The most recent of these is Alcade. As previously discussed, the Alcade classification system is based on the roughness of the sample. The roughness results of this experiment have been compared to the current CIE system in Section 6.6.1 and to the parameters of the Alcade system in Section 6.6.2.

\subsubsection{Correlation to the CIE System}

The CIE reflection parameters are based on the light reflection characteristics of the sample only. In order to investigate the relationship of the surface macro-roughness characteristics to the reflection parameters, Pearson correlation coefficients were established between the roughness data and the CIE parameters. All of the measured characteristics of the roughness were used in this correlation. The correlation was also performed at two $\alpha$ levels, $1^{\circ}$ and $45^{\circ}$. These correlation results are presented in Table 23.

Table 23 - Correlation of Surface Roughness Characteristics to CIE Reflection Parameters

\begin{tabular}{|c|c|c|c|c|c|c|c|c|}
\hline \multicolumn{9}{|c|}{ Correlations (Pearson) } \\
\hline $\begin{array}{ll}\mathrm{Q} 0 & \alpha 1^{\circ} \\
\mathrm{S} 1 & \alpha 1^{\circ} \\
\mathrm{S} 2 & \alpha 1^{\circ}\end{array}$ & $\begin{array}{r}\text { RoughWT } \\
-0.219 \\
-0.251 \\
-0.323\end{array}$ & $\begin{array}{r}\text { RoughAT } \\
-0.303 \\
-0.140 \\
-0.184\end{array}$ & $\begin{array}{r}\text { CDWT } \\
-0.025 \\
-0.176 \\
-0.298\end{array}$ & $\begin{array}{r}\text { CDAT } \\
-0.008 \\
-0.113 \\
-0.315\end{array}$ & $\begin{array}{l}\text { Ave R } \\
-0.271 \\
-0.199 \\
-0.258\end{array}$ & $\begin{array}{l}\text { Ave CD } \\
-0.022 \\
-0.185 \\
-0.389\end{array}$ & $\begin{array}{r}\text { Area } \\
-0.242 \\
-0.223 \\
-0.389\end{array}$ & $\begin{array}{l}\text { Angle } \\
-0.339 \\
-0.170 \\
-0.040\end{array}$ \\
\hline $\begin{array}{l}\mathrm{Q} 0 \propto 45^{\circ} \\
\text { SI } \alpha 45^{\circ} \\
\text { S2 } \alpha 45^{\circ}\end{array}$ & $\begin{array}{r}\text { RoughWT } \\
0 \quad 0.075 \\
\circ \quad-0.290 \\
-0.204\end{array}$ & $\begin{array}{r}\text { RoughAT } \\
-0.054 \\
-0.249 \\
-0.006\end{array}$ & $\begin{array}{r}\text { CDWT } \\
0.364 \\
-0.260 \\
-0.375\end{array}$ & $\begin{array}{r}\text { CDAT } \\
0.604 \\
-0.300 \\
-0.512\end{array}$ & $\begin{array}{l}\text { Ave R } \\
0.008 \\
-0.277 \\
-0.104\end{array}$ & $\begin{array}{r}\text { Ave CD } \\
0.609 \\
-0.355 \\
-0.560\end{array}$ & $\begin{array}{r}\text { Area } \\
0.546 \\
-0.308 \\
-0.454\end{array}$ & $\begin{array}{r}\text { Angle } \\
-0.256 \\
-0.037 \\
0.308\end{array}$ \\
\hline
\end{tabular}


In these results, there appears to be no strong correlation of the surface roughness data to the reflection parameters. The strongest consistent relationship seems to be the facet area. The relationship of the facet area to the $\mathrm{q}_{0}$ parameter is shown in Figure 66 .

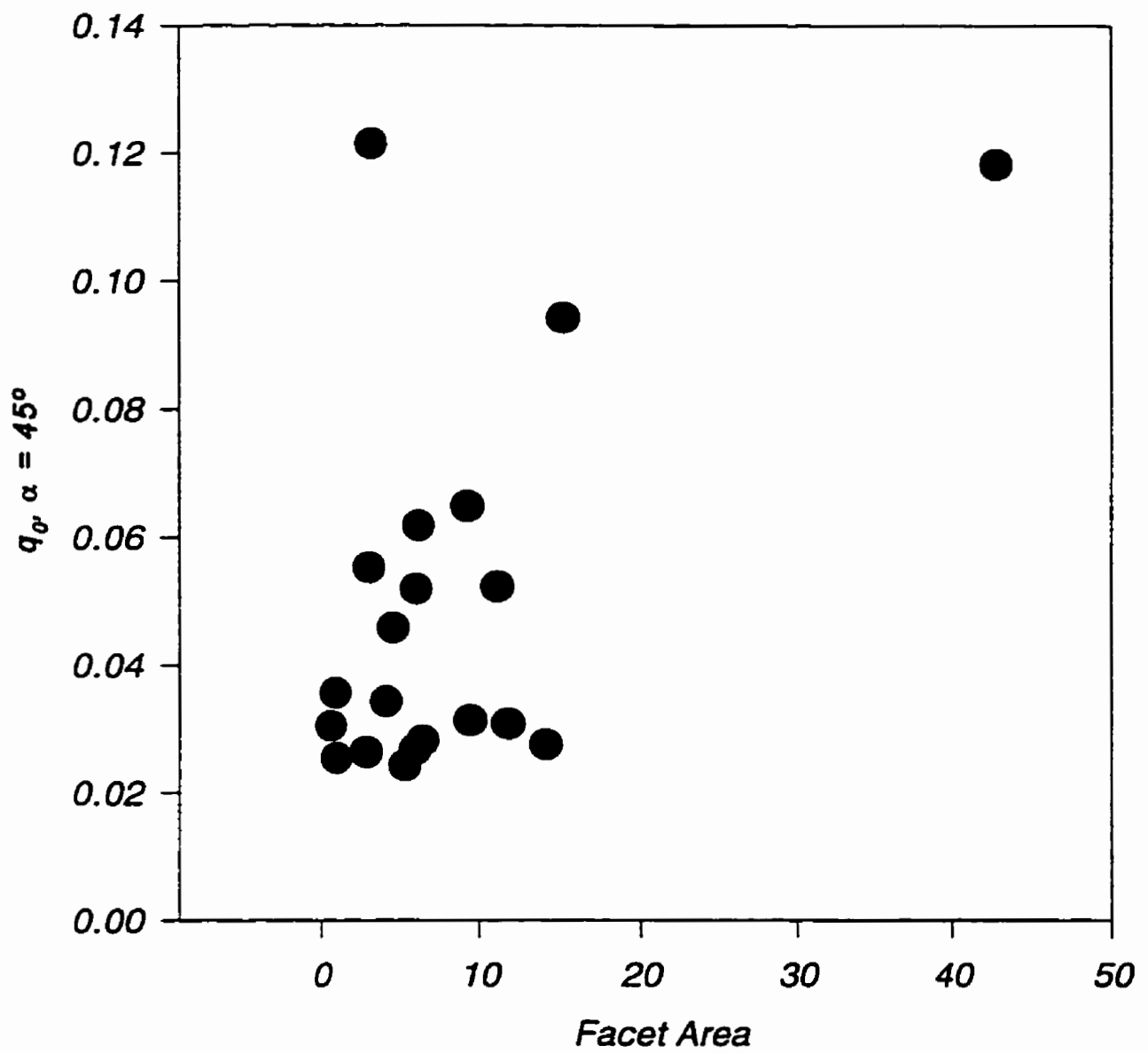

Figure 66 - Correlation of $q_{0}$ to the Facet Area 


\subsubsection{Correlation to the Alcade Criteria}

In the Alcade system, the parameter $A_{D}$ is based on the macro roughness of the sample.

The other parameters, $A_{11}$ and $A_{12}$, are used to account for the micro roughness. It is unknown whether these parameters were determined based on actual measurements of the roughness or whether they were chosen and then qualitatively related to the surface roughness.

For the reflection results from this experiment, Pearson correlation coefficients were established for the Alcade parameters and the roughness measurement. The method used for this correlation was the same as that used for the CIE parameters. These results are presented in Table 24.

Table 24 - Correlation of Surface Roughness Characteristics to Alcade Reflection Parameters

\begin{tabular}{|c|c|c|c|c|c|c|c|}
\hline \multicolumn{8}{|c|}{ Correlations (Pearson) } \\
\hline $\begin{array}{lr} & \text { RoughwT } \\
\text { AD } \propto 1^{\circ} & -0.325 \\
\text { Al1 } \alpha 1^{\circ} & -0.187 \\
\text { Al2 } \alpha 1^{\circ} & 0.111\end{array}$ & $\begin{array}{r}\text { RoughAT } \\
-0.192 \\
-0.040 \\
0.005\end{array}$ & $\begin{array}{r}\text { CDWT } \\
-0.304 \\
-0.101 \\
0.287\end{array}$ & $\begin{array}{r}\text { CDAT } \\
-0.234 \\
-0.013 \\
0.096\end{array}$ & $\begin{array}{l}\text { Ave R } \\
-0.263 \\
-0.113 \\
0.057\end{array}$ & $\begin{array}{l}\text { Ave CD } \\
-0.343 \\
-0.075 \\
0.249\end{array}$ & $\begin{array}{r}\text { Area } \\
-0.337 \\
-0.130 \\
0.127\end{array}$ & $\begin{array}{l}\text { Angle } \\
-0.140 \\
-0.158 \\
-0.015\end{array}$ \\
\hline $\begin{array}{lr} & \text { RoughWT } \\
\text { AD } \alpha 45^{\circ} & -0.303 \\
\text { A11 } \alpha 45^{\circ} & -0.342 \\
\text { A12 } \alpha 45^{\circ} & 0.148\end{array}$ & $\begin{array}{r}\text { RoughAT } \\
-0.252 \\
-0.299 \\
0.264\end{array}$ & $\begin{array}{r}\text { COWT } \\
-0.270 \\
-0.263 \\
0.054\end{array}$ & $\begin{array}{r}\text { CDAT } \\
-0.253 \\
-0.420 \\
-0.035\end{array}$ & $\begin{array}{l}\text { Ave R } \\
-0.284 \\
-0.329 \\
0.215\end{array}$ & $\begin{array}{l}\text { Ave CD } \\
-0.333 \\
-0.430 \\
0.014\end{array}$ & $\begin{array}{r}\text { Area } \\
-0.291 \\
-0.373 \\
0.043\end{array}$ & $\begin{array}{r}\text { Angle } \\
-0.067 \\
-0.010 \\
0.254\end{array}$ \\
\hline
\end{tabular}

Like the CIE results, there are no strong relationships of the data to the surface macro roughness characteristics. As an example of this, the relationship of the $A_{D}$ parameter to the average surface roughness is shown in Figure 67. 


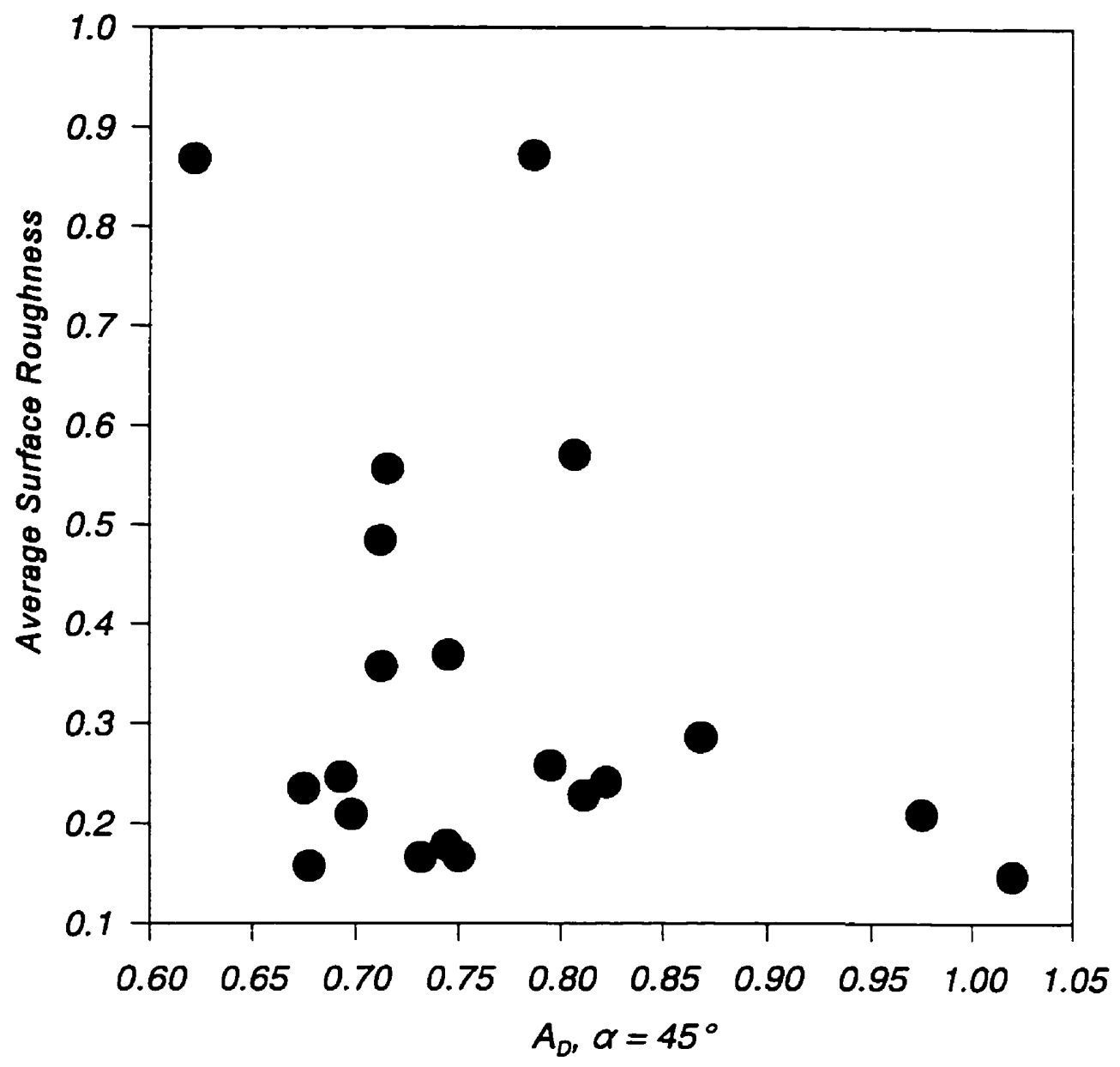

Figure 67 - Correlation of $A_{D}$ to Average Surface Roughness

\subsection{Conclusion}

The surface macro roughness and the surface autocorrelation distance was established for the 20 pavement samples used in the reflection characteristics experiment. The 
relationship of the surface roughness to the pavement recipe and to the CIE and Alcade reflection indices was also investigated.

A correlation of the percent coarse aggregate to the surface roughness was found in the material recipe investigation.

There were no significant relationships found in the reflection indices investigation. Due to this result and that of the reflection experiment, the applicability of either the CIE system and the Alcade system could not be determined from a relationship to the physical properties of the sample. An application experiment and investigation into usefulness and accuracy these two pavement reflection classification systems is required, which is outside the boundaries of this research.

The surface roughness data will be used to investigate the different possibilities for mathematically describing the reflection data for each of the samples in the experiment which is discussed in Chapter 7. 


\section{Analytical Modeling of Pavement Reflection}

The current systems for using pavement reflection data are mostly empirical. Look-up tables and data generalizations have been the only methods for dealing with this volume of data. With the addition of the observation angle data. The process has become even more cumbersome. An analytical system would greatly improve the ease of using this data.

Previous work performed in the development of an analytical model has been limited. Two models exist which were developed at the University of Waterloo but no fully adequate solution has been found.

In the development of an analytical solution, it is desirable to attempt to relate some of the aspects of the pavement surface itself to the reflection character of each sample. To accomplish this, two methodologies have been investigated. These are an adaptation of a physical optics method used for radio waves, and a method based on the work of Vermeulen[1975], separating the diffuse and specular portions of the reflection profile.

These two methods and the previous work in the development of an analytical model are highlighted in the following discussions.

\subsection{Previous Models of Pavement Reflection}

Two models have been developed to mathematically describe pavement reflection. The 
first, presented by White [1994], is a mathematical system developed to represent the $\alpha=1^{\circ}$ reflection tables. The second, developed in Gibbons and Adrian[1994], is a brute force mathematical model developed to represent the change in the reflection properties of the samples based on the $\alpha=1^{\circ}$ data. The original plan for these two models was to model all of the reflection data using a combination of the two systems. However, there are flaws in the development of both which makes their application limited.

The White model consists of a brute force mathematical method for fitting the r-table data. White fit a basic mathematical equation to all of the r-table data. This basic model was determined to be a combination of a Guassian distribution and a step function as shown in Equation 43.

$$
r=f(\gamma)=C_{1} \cdot e^{C_{2} \cdot\left(\gamma+C_{3}\right)^{2}}+\left(1-C_{4} \cdot e^{-C_{5} \cdot \gamma^{2}}\right)
$$

The parameters from this basic model were then extrapolated across the $\beta$ and the RClass data. The resulting parameters are shown in Equation 44.

$$
\begin{aligned}
& C_{1}=\left(-50 \cdot(R-0.8)^{2}+47\right) \cdot \beta^{5}-\left((-2 \cdot R-0.6)^{2}+2.2\right) \cdot \beta+\left(300 \cdot(R-1.25)^{2}+100\right) \\
& C_{2}=0.5 \\
& C_{3}=(0.4 \cdot R-0.09) \cdot \beta^{5}-(0.015 \cdot R-0.0053) \cdot \beta+(1.9 \cdot R-0.64) \\
& C_{4}=(30 \cdot R+23) \cdot \beta^{5}-(1.9 \cdot R+1.35) \cdot \beta+(131 \cdot R-113) \\
& C_{5}=\left(-0.012 \cdot(R-0.75)^{2}+0.012\right) \cdot \beta^{5}+\left((0.0004 \cdot R-0.5)^{2}+0.00045\right) \cdot \beta+\left(-0.02 \cdot(R-0.66)^{2}+0.022\right)
\end{aligned}
$$

Where $\mathrm{R}$ is the $\mathrm{R}$-Class rating. 
This resulting model is very complex. White states that the average error in the model is from 6 to $38 \%$ with errors as large as $400 \%$. White also shows the comparison of the model to the data. The results show extensive inaccuracies at the high $\gamma$ values.

This methodology has some flaws in it. As the parameters for the model were developed, White did not refer back to the original data as he set each equation. This is likely why the model does not match the data very well. Similarly, the use of $R$ as a descriptor for the model is of little practical value. A better descriptor would be SI. This would allow the designer to input a measured or a standard $\mathrm{S} 1$ value and retrieve the $\mathrm{r}$ table regardless of the R-Class rating.

The Gibbons and Adrian model is based on the measurements of one sample, Ontario L7-3. At the time of the development of the model, it was felt that a model of a multiplier to the existing $\alpha=1^{\circ}$ r-tables would be better than modeling the absolute values of the results. The model tabulated in this way can be used in a more general manner than the absolute value model. The multiplier method was developed by basing the reading at $\alpha>1^{\circ}$ on the reading for $\alpha=1^{\circ}$. This was performed for all combinations of $\beta$ and $\gamma$. The formulation of the scaling is:

$$
r f a c t(\alpha, \beta, \gamma)=\frac{r(\alpha, \beta, \gamma)}{r(\beta, \gamma)}
$$

This formulation causes the data for $\alpha=I^{\circ}$, to be equal to one. From that point, the data rises or falls generally to a steady state at high $\alpha$, representing the trend in the data 
toward diffuse reflection.

The model methodology used for this process was a mathematical model based on the summation of an inverse exponential, and a hyperbolic tangent. The resulting form is:

$$
\operatorname{factor}(\alpha, \beta, \gamma)=\frac{1}{e^{a \cdot(\alpha-1)}}+b \cdot \tanh (c \cdot(\alpha-1))
$$

The parameters of this equation, $a, b$, and $c$ depend on $\gamma$ and $\beta$. These parameters are:

$$
\begin{aligned}
& a=4.849 \cdot 10^{-9} \cdot \gamma^{1.578} \cdot(\beta-91)^{2}+0.00139 \\
& b=1.229 \cdot 10^{-5} \cdot(\gamma-73.92) \cdot \beta+\left(-1.731 \cdot 10^{-5} \cdot \gamma^{2}+0.2360\right) \\
& \text { and } \\
& c=1.020
\end{aligned}
$$

This model has an $r^{2}$ correlation factor of .978 to the Ontario L7-3 data.

This method of calculating the reflection properties of the pavement provides a very good solution for the single sample tested. The obvious problem with the Gibbons and Adrian model is the lack of applicability across many different types of samples. The data used for this model is also a result of the earlier design of the gonioreflectometer which was discussed in Chapter 4. It was found through later redesign and calibration of the system that this data has flaws.

Both of these modeling methods show some difficulties. The first model did not use 
parameters which are related to the pavement surface itself. It used categorization parameters which have little relationship to the pavement surface. The model of Gibbons and Adrian is based on a single sample and has limited relationship to the entire data set. Other models will be considered in the representation of the data.

\subsection{Lambertian Reflection Relationship}

One of the key aspects of the data as presented is that the reflection data approaches perfectly diffuse reflection or Lambertian reflection. To more fully develop a model, the relationship of the data to Lambertian reflection must be clearly established. As previously described, a Lambertian surface is defined as a surface which appears equally bright in all directions. This means that the luminance of the surface is the same regardless of the observation angle. The reflected luminance for this perfectly diffusing surface varies only with incident luminous intensity. This intensity reduces with the cosine of the angle between the normal of the surface and the angle of incidence. If a pavement surface behaved like a Lambertian surface, $\gamma$ would be the only angle which would influence the luminance of the surface. The luminance to $\gamma$ relationship would resemble a cosine curve representing the reduction in the intensity. There would be no $\beta$ and $\alpha$ to luminance relationships. These would be represented on a graph as horizontal lines. Previously, it was discussed that the data shows a trend toward Lambertian reflection at high levels of $\alpha$. Figure 68 shows the relationship of the observation angle to the pavement luminance for each R-Class. The $95 \%$ Confidence Interval for each data point is also shown. In this figure, a horizontal line has been drawn through the $\alpha=60^{\circ}$ 
data point for each R-Class curve. When the line is outside of the $95 \%$ confidence bars the surface no longer behaves as a perfect diffuser. This angle is approximately $20^{\circ}$ for road classes $R 1, R 2$ and $R 3$, and approximately $30^{\circ}$ for the $R 4$ class.

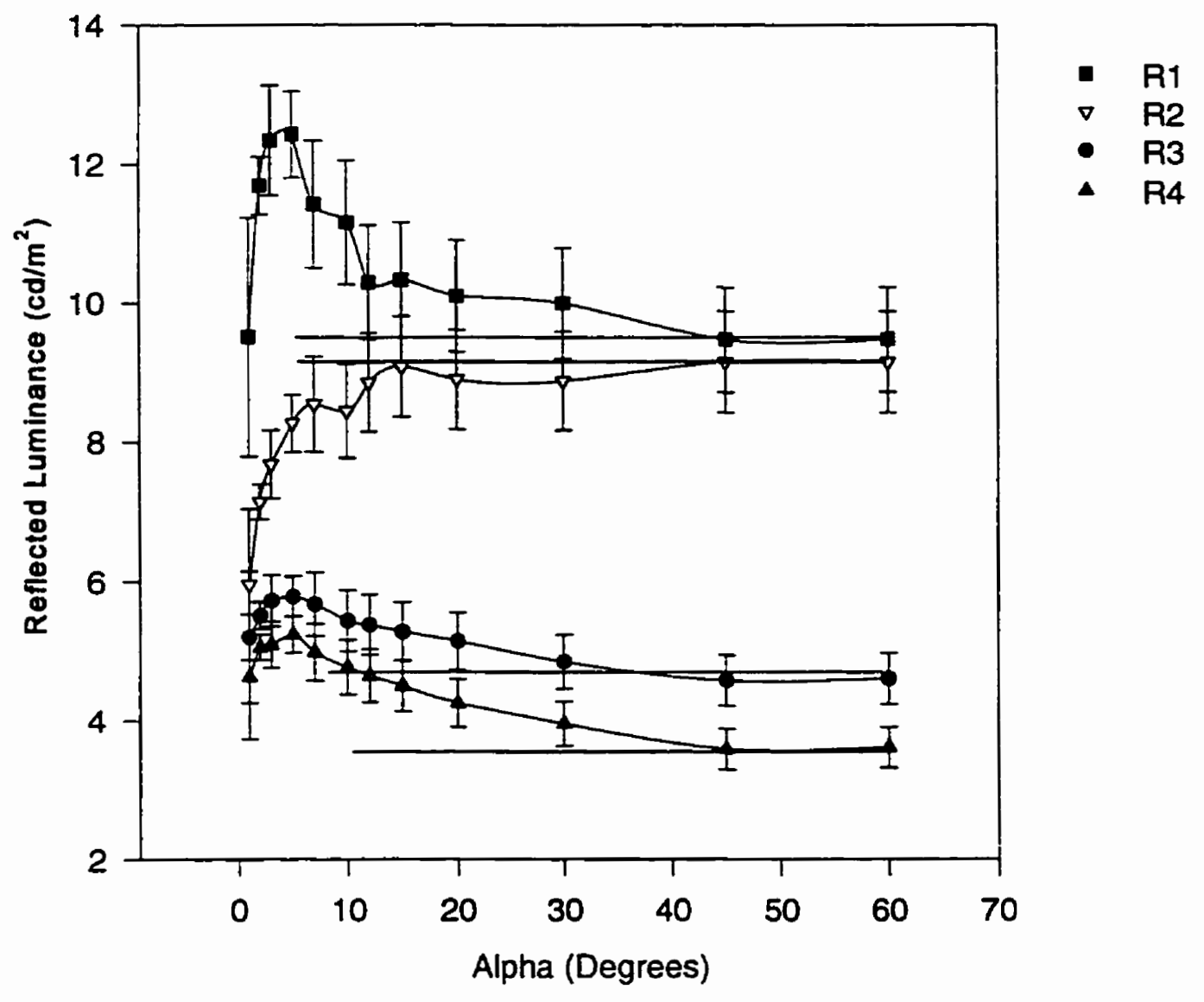

Figure 68 - $\alpha$ versus Reflected Luminance with the Lambertian Reflection Relationship $\left(\beta=0^{\circ}, \gamma=0^{\circ}\right)$

In order to further investigate the Lambertian trend, the changes in the luminance with 
the changes in $\gamma$ and $\beta$ were plotted in Figures 69 and 70. The $\alpha$ value in these plots is $60^{\circ}$, the highest value of $\alpha$ tested. The solid lines without data points in Figure 69 represent the best fit lines for a cosine function. The regression coefficients for the data to the cosine function is $0.90,0.99,0.95$, and 0.78 for the four road classes respectively. In Figure 70 , the effect of $\beta$ is shown with the desired effect being represented by a solid line. The data in this graph is the ratio of each data point to the average of all of the data points for that road class. There is an average change from $+6 \%$ to $-4 \%$ across the range of the $\beta$ readings. This is within the standard error of the experiment. From these comparisons, it can be seen that Lambertian reflection can be used to model the reflection of light from pavement surfaces within limits. The influences of the $\gamma$ and $\beta$ behave in a manner which would be expected from a Lambertian surface which gives further evidence in support of this model. 


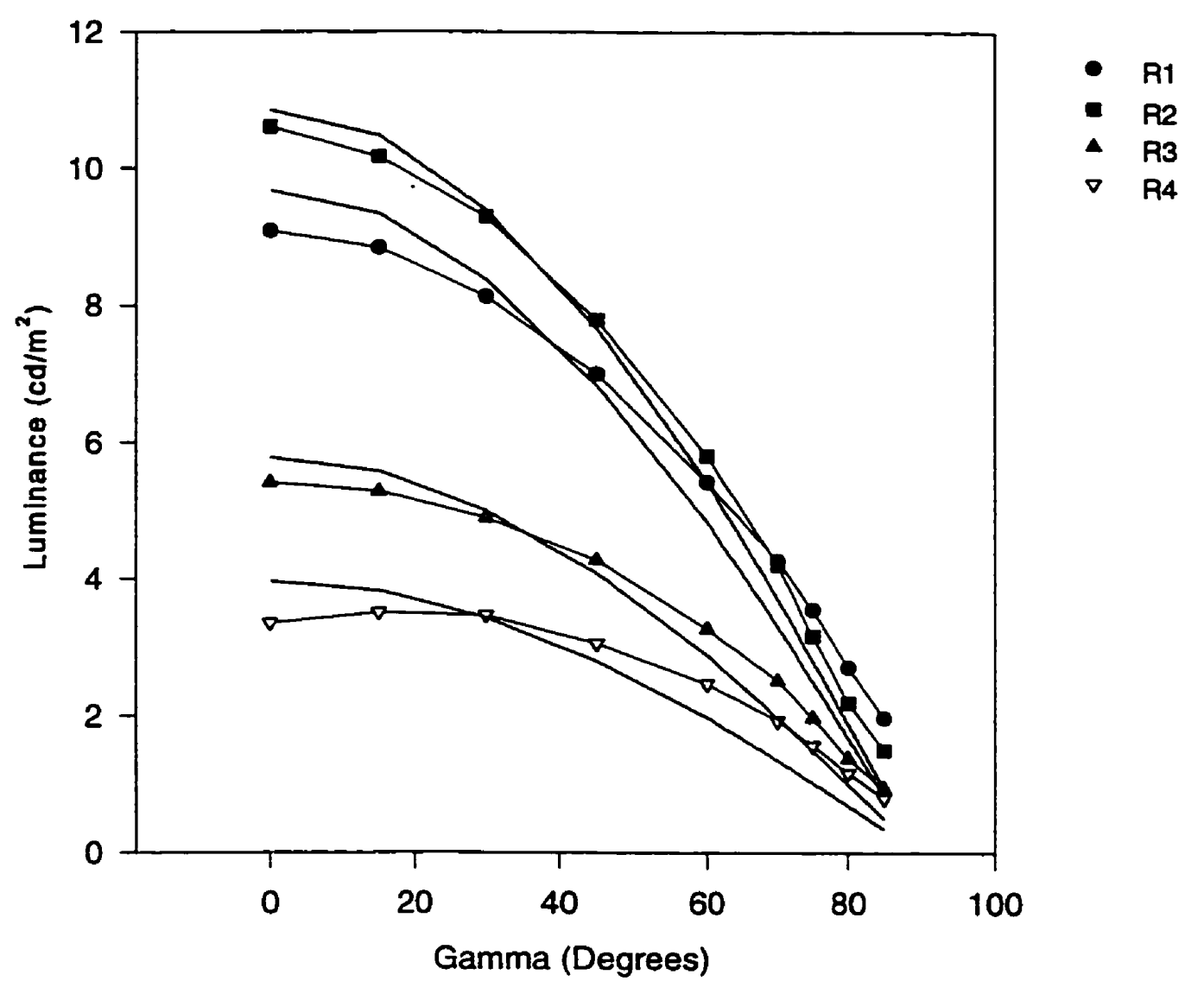

Figure 69 - Pavement Luminance versus $\gamma$ with Lambertian Reflection Relationship with the Reduction in Incident Intensity $\left(\alpha=60^{\circ}, \beta=0^{\circ}\right)$ 


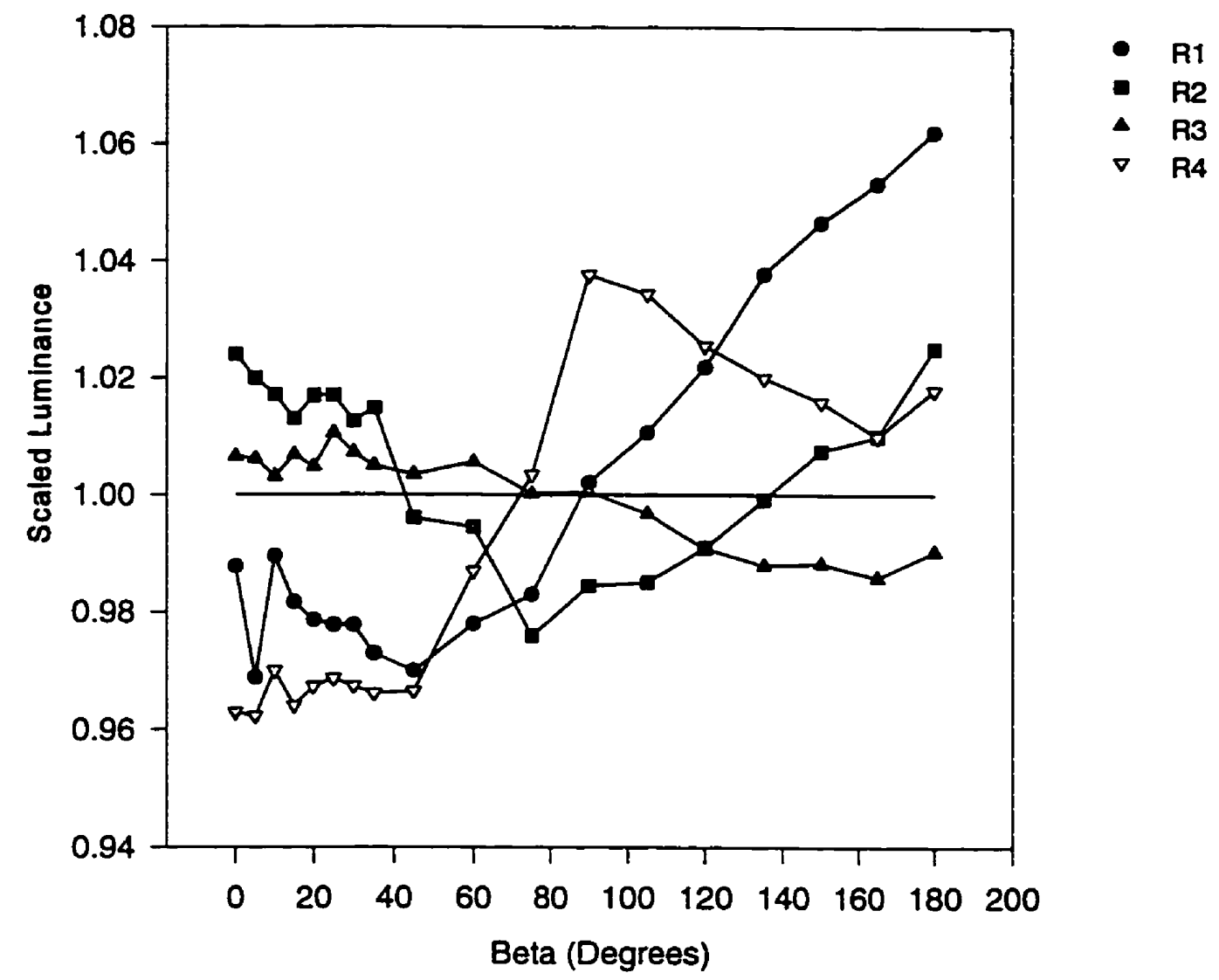

Figure 70 - Pavement Luminance versus $\beta$ with Lambertian Reflection Profile ( $\alpha=60^{\circ}$, $\left.\gamma=0^{\circ}\right)$

As was discussed earlier, the reflection from a pavement surface is defined as compound, consisting of both a specular and a diffuse component. In order to see this effect, a new angle, $\mu$, was defined as the angle between the angle of observation and the specular angle which is equal and opposite to the angle of reflection. Thus $\mu=0^{\circ}$ is representative of specular reflection. A scatter plot of the reflection factor, $q$ against $\mu$ is shown in Figure 71. The important features of this graph are, of course, the flat reflection profile 
at the extremes of the diagram and the peak at the central $\mu=0^{\circ}$ location. The flat section of this graph represents diffuse reflection and the peak represents specular reflection.

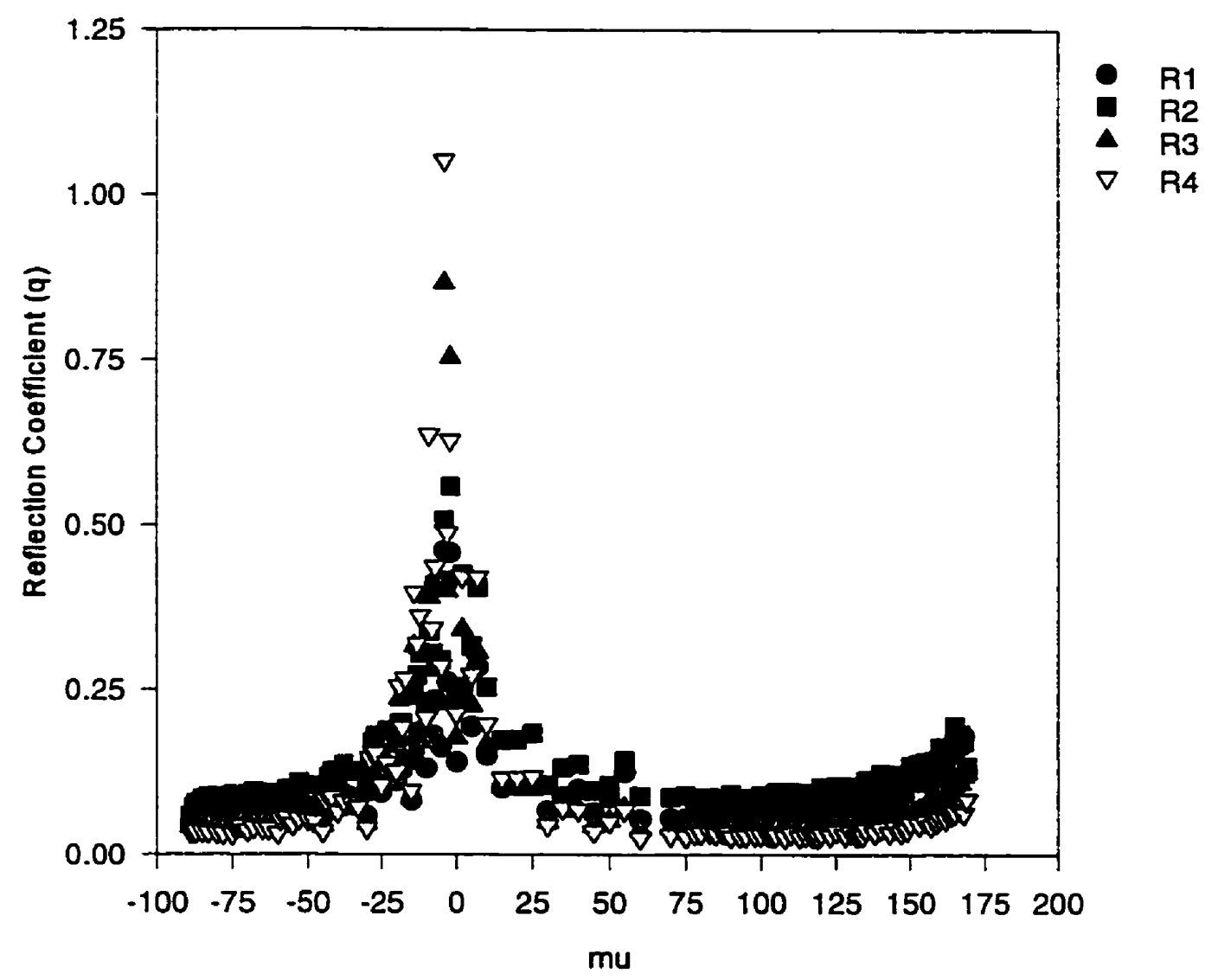

Figure 71 - Reflection Coefficient, q, versus $\mu$ for all R-Classes

This relationship in the reflection data to Lambertian reflection and to the nature of the compound reflection is a critical aspect of the other modeling methods which were investigated. 


\subsection{Facet Model of the Reflection Profile}

A modeling method, called the facet model, is used to describe the specular and the diffuse components of the reflection. The term facet refers to the individual reflecting surface in the pavement itself. This was described in Chapter 3. This method uses the orientation and the size of these facets as the basis of describing each of the two components of the reflection profile. Vermuelen [1975] conducted a series of experiments considering the reflection of light from rough surfaces and used a facet model to aid in the description of the reflection. He considered the specular and the diffuse components of the reflection in terms of the total reflection character of the surface, using polarizing filters to separate and measure each of these components.

\subsubsection{Model Development}

Vermeulen developed his interpretation of the facet model based on the assumption that only the small facets in the surface which are oriented properly will reflect specularly. These reflecting facets are those which have their surface normals oriented at the bisector of the angles of observation and incidence. The model is then based on a relationship of the specular portion of the reflection to the change in the reflective area and to the angular components. This method shows that the luminous flux reflected from the surface is proportional to the area of each reflected facet and the number of the facets which are oriented properly. Equation 48 shows this relationship as defined by Vermeulen. 


$$
\Theta_{s}(\epsilon, i)=\rho(i) \cdot N(\epsilon) \cdot \eta(\epsilon, i) \cdot \Theta_{i} \cdot \frac{\cos (i)}{\cos (\epsilon-i)} \cdot A_{f}
$$

In this equation, $\Theta_{i}$ is the incidence luminous flux on the surface, $A_{f}$ is the average area of the reflecting facets and $\rho(\mathrm{i})$ is the reflectivity of the pavement. $N(\epsilon)$ describes the number of facets which are oriented properly for specular reflection.

In Equation 48, $\epsilon$ is the angle between the normal of the reflecting facet and the normal of the entire surface and and $i$ is the angle between the angle of incidence and observation to the normal of the reflecting facet. These angles are shown in Figure 72. In Figure 72, the reflection at a point, $\mathrm{P}$, of light from a source at the apex of the angle $\gamma$ to an observer defined by $\alpha$.

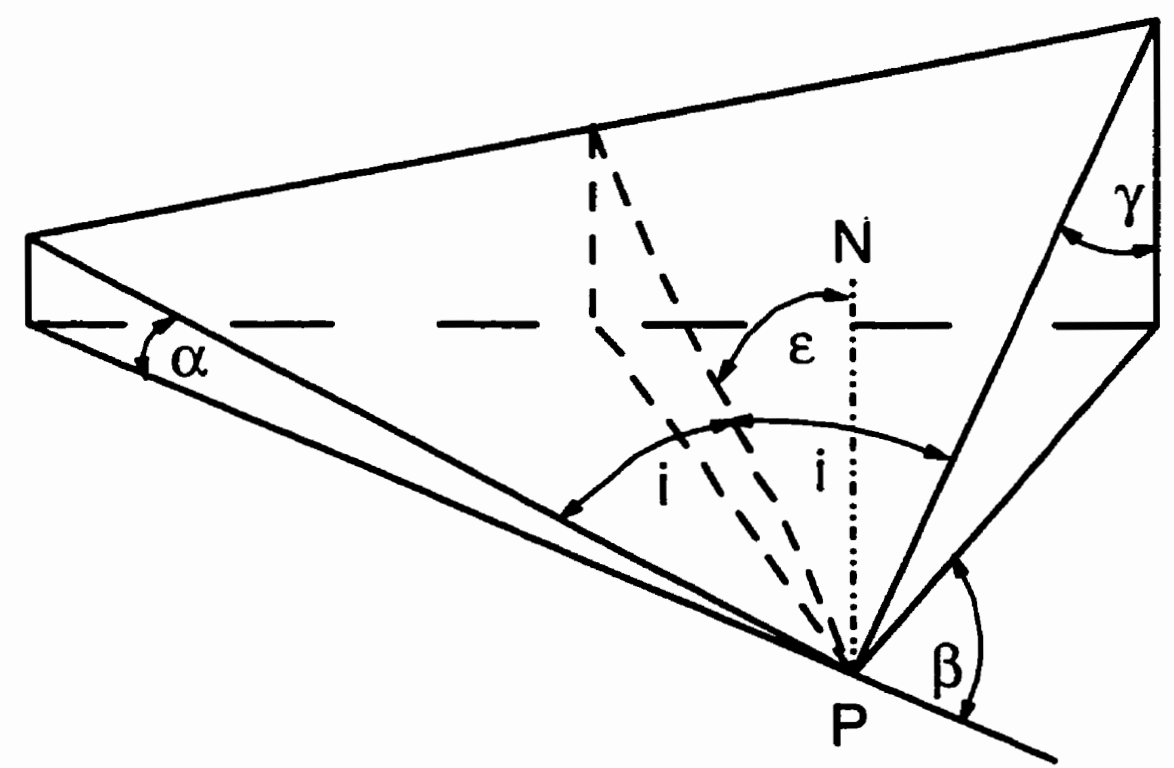

Figure 72 - Angles for Facet Model Derivation from Vermeulen [1975]

From Figure 72, the relationships of the angles $\epsilon$ and $i$ to the typical angles of $\alpha, \beta$, and $\gamma$ 
were derived by Kebschull [1969]. These relationships are shown in Equation 49.

$$
\begin{gathered}
\cos (2 i)=\sin (\alpha) \cdot \cos (\gamma)-\sin (\gamma) \cdot \cos (\alpha) \cdot \cos (\beta) \\
\cos (\epsilon)=\frac{\sin (\alpha)+\cos (\gamma)}{2 \cos (i)}
\end{gathered}
$$

Also in Equation 48, $\eta(\epsilon, \mathrm{i})$ is a shadowing function which describes the shadowing of one portion of the surface onto another section of the surface. The requirement for this shadowing function is to estimate the change in the number of facets which are available for reflection. This shadowing function is based both on the roughness of the surface and on the angle of incidence. Figure 73 shows an example of this shadowing effect.

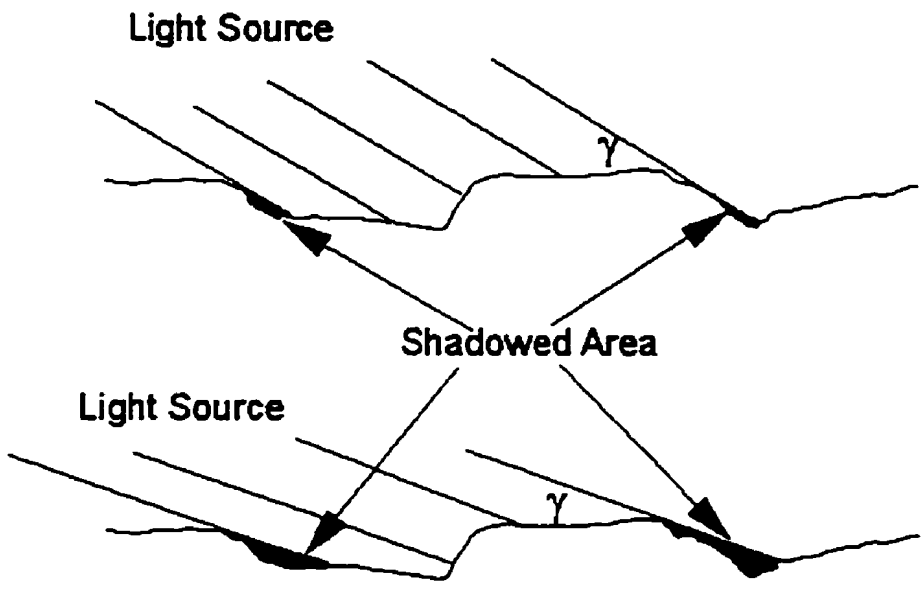

Figure 73 - Shadowing Effect

In order to use the facet model, each of the portions of this equation must be developed.

The other reflection component, the diffuse portion, is calculated based on Lambert's 
Law. A function which describes the deviation of the reflection from Lambert's Law and a shadowing function which describes the change in the reflecting area were added to the general formulation. This relationship for the diffuse component of the reflected flux, $\boldsymbol{\theta}_{\mathrm{d}}$, is shown in Equation 50.

$$
\Theta_{d}\left(\varphi_{1}, \varphi_{2}\right)=\frac{\rho_{d}}{\pi} \cdot D\left(\varphi_{1}, \varphi_{2}\right) \cdot \eta_{2}\left(\varphi_{1}, \varphi_{2}\right) \cdot \Theta_{i} \cos (\varphi) \cdot \Delta \omega
$$

In this equation, $\varphi_{1}$ and $\varphi_{2}$, are the angles of reflection and incidence relative to the surface normal and are shown in Figure 74.

In order to use the facet model, each of the portions of this equation must be developed.

The other reflection component, the diffuse portion, is calculated based on Lambert's Law. A function which describes the deviation of the reflection from Lambert's Law and a shadowing function which describes the change in the reflecting area were added to the general formulation. This relationship for the diffuse component of the reflected flux, $\theta_{d}$, is shown in Equation 50.

$$
\Theta_{d}\left(\varphi_{1}, \varphi_{2}\right)=\frac{\rho_{d}}{\pi} \cdot D\left(\varphi_{1}, \varphi_{2}\right) \cdot \eta_{2}\left(\varphi_{1}, \varphi_{2}\right) \cdot \Theta_{i} \cos (\varphi) \cdot \Delta \omega
$$

In this equation, $\varphi_{1}$ and $\varphi_{2}$, are the angles of reflection and incidence relative to the surface normal and are shown in Figure 74. 


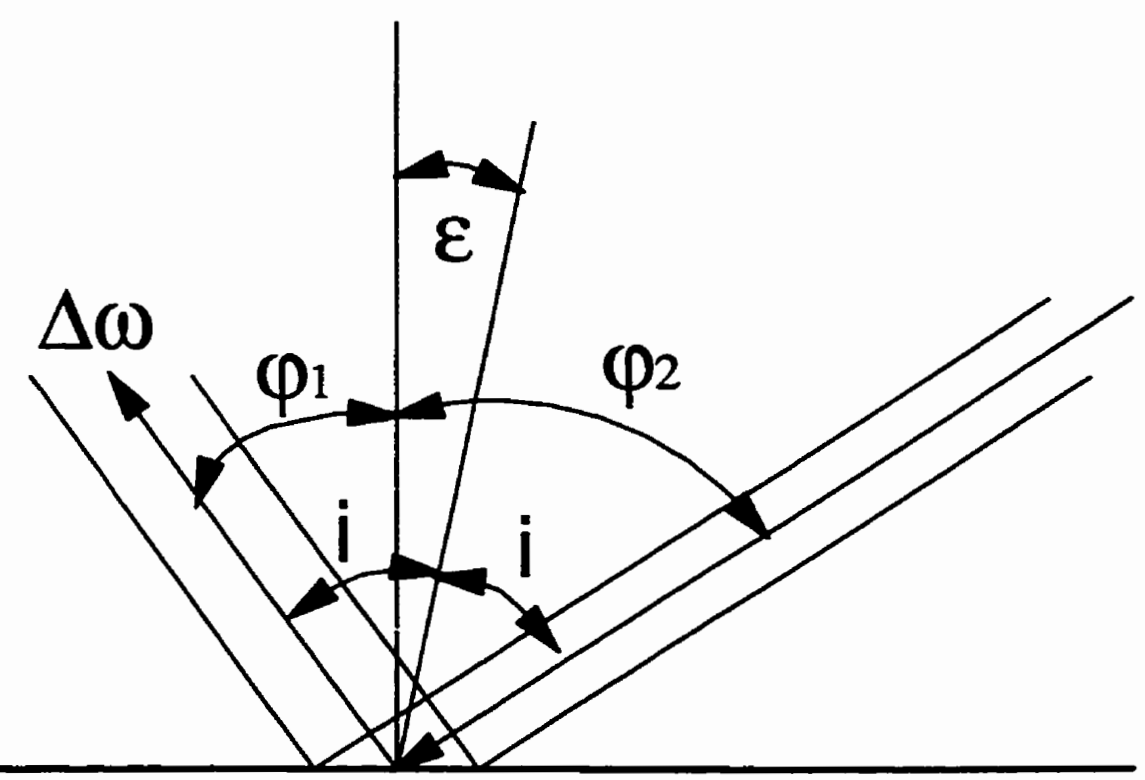

Figure 74 - Angle Definition for the Diffuse Reflection Model

The angles $\varphi_{1}$ and $\varphi_{2}$ are both measured as positive in the counter clockwise direction and negative in the clockwise direction from the normal to the surface. Obviously, $\varphi_{1}$ is equal to $90-\alpha$ and $\varphi_{2}$ is equal to $\gamma \cdot \rho_{d}$ is the diffuse reflectivity of the surface and $\Delta \omega$ is the acceptance aperture of the measurement instrument. $\mathrm{D}\left(\varphi_{1}, \varphi_{2}\right)$ is the diffuse reflection factor which is applied to account for the deviation of the reflection from Lambert's law and $\eta_{2}\left(\varphi_{1}, \varphi_{2}\right)$ is the diffuse shadowing function. Vermeulen states that in general, the specular and the diffuse shadowing functions are not similar. The factor, $\Theta_{i} \cos (\varphi)$, represents the incident luminous intensity adjusted by Lambert's cosine law. In this calculation, the factor $\varphi$ represents the maximum absolute value of $\varphi 1$ and $\varphi 2$.

Since according to the defintion, the diffuse shadowing function and the diffuse distribution function both depend on the incident and observation angles, these functions would be indistinguishable in the measurements. Vermeulen then defined the diffuse 
distribution function as $D(\varphi)=D\left(\varphi_{1}=\varphi_{2}\right)$ as shown in Equation 51 .

$$
D\left(\varphi_{1}=\varphi_{2}\right)=\frac{\Theta_{d}\left(\varphi_{1}=\varphi_{2}\right)}{\Theta_{d}(0,0) \cdot \cos (\varphi)}
$$

The diffuse shadowing function was then defined as shown in Equation 52.

$$
\eta_{2}\left(\varphi_{1}, \varphi_{2}\right)=\frac{\Theta_{d}\left(\varphi_{1}, \varphi_{2}\right)}{\Theta_{d}\left(\varphi_{1}=\varphi_{2}\right)}
$$

These two assumptions allow the nature of the diffuse functions to be determined independently.

It should be noted that the effect of the angle $\beta$ was not included in the diffuse portion of Vermeulen's development but, as shown earlier, $\beta$ has no impact on diffuse reflection.

Vermeulen used an experimental set-up to attempt to describe the specular and diffuse reflection functions. He used a polarizing filter to discern these two portions of the reflection. This was possible since the specular reflection portion is polarized perpendicularly with the surface of the test sample. Vermeulen used a piece of sandpaper as the test sample and was able to distinguish between the two components. He also showed that the formulation of the facet model did apply to the data. Vermeulen did have difficulties with the measurements and felt that the actual definition of the diffuse functions required further investigations. Any further experimentation in this field has not been widely published. 


\subsubsection{Model Application}

The applicability of Vermuelen's methodology to the reflection data in this research was not performed easily. A polarizing filter was not considered for use in the pavement reflection experiment. This meant that the variables and the functions in this model had to be developed mathematically based on the results of the experiment.

The first step in this application was the development of the diffuse portion of the reflection nature which required the development of $D(\varphi)$ and $\eta_{2}\left(\varphi_{1}, \varphi_{2}\right)$.

The function $\mathrm{D}(\varphi)$ was determined based on the data where $\varphi_{1}=\varphi_{2}$, or in other words, when $\alpha=90-\gamma$. This occured when the light source and the detector were in the same position. The only occurrence of this was at $\beta=180^{\circ}$. The $D(\varphi)$ function was developed for every sample and is shown in Figure 75. 


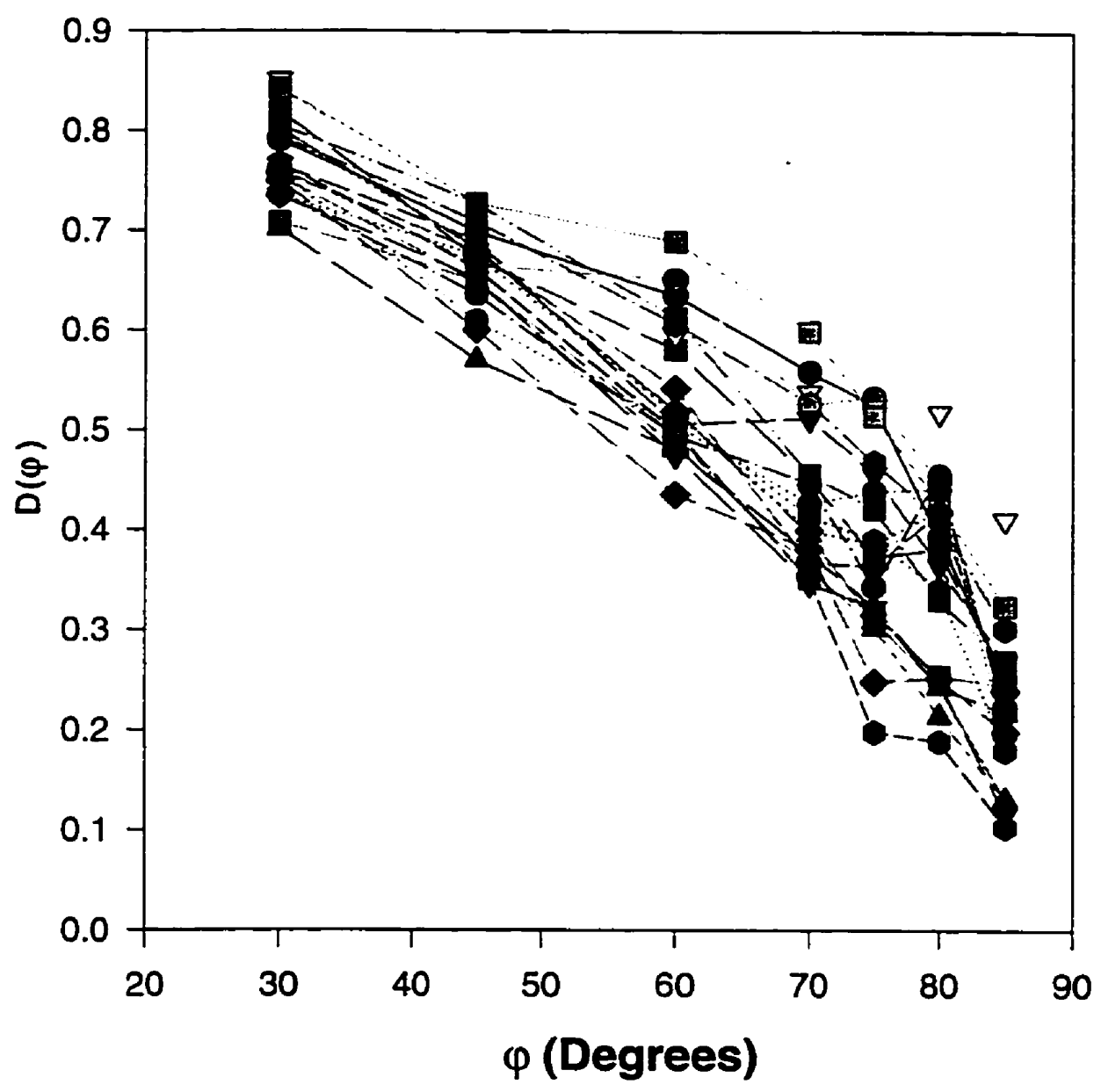

Figure $75-D(\varphi)$ for All Samples

Figure 75 does not have a legend due to the number of data points represented: all the samples measured are presented. There seems to be very little dependence of the $D(\varphi)$ function of the sample type. A mathematical equation to fit this function was developed as follows:

$$
D(\varphi)=1-0.00307 \cdot \varphi^{1.231}
$$


The next step was the development of the shadow function, $\eta_{2}\left(\varphi_{1}, \varphi_{2}\right)$. This function was developed based on the $\beta=180^{\circ}$ data. Using the developed $D(\varphi)$ function, $\eta_{2}\left(\varphi_{1}, \varphi_{2}\right)$ was calculated as the ratio of the measured reflectance $q\left(\alpha, \beta-180^{\circ}, \gamma\right)$ and $D(\varphi)$ where $\varphi=$ $\gamma$. For this development, the entire set of $\alpha$ and $\gamma$ combinations were used. This resulting function is shown in Figures 76 to 79 for each R-Class.

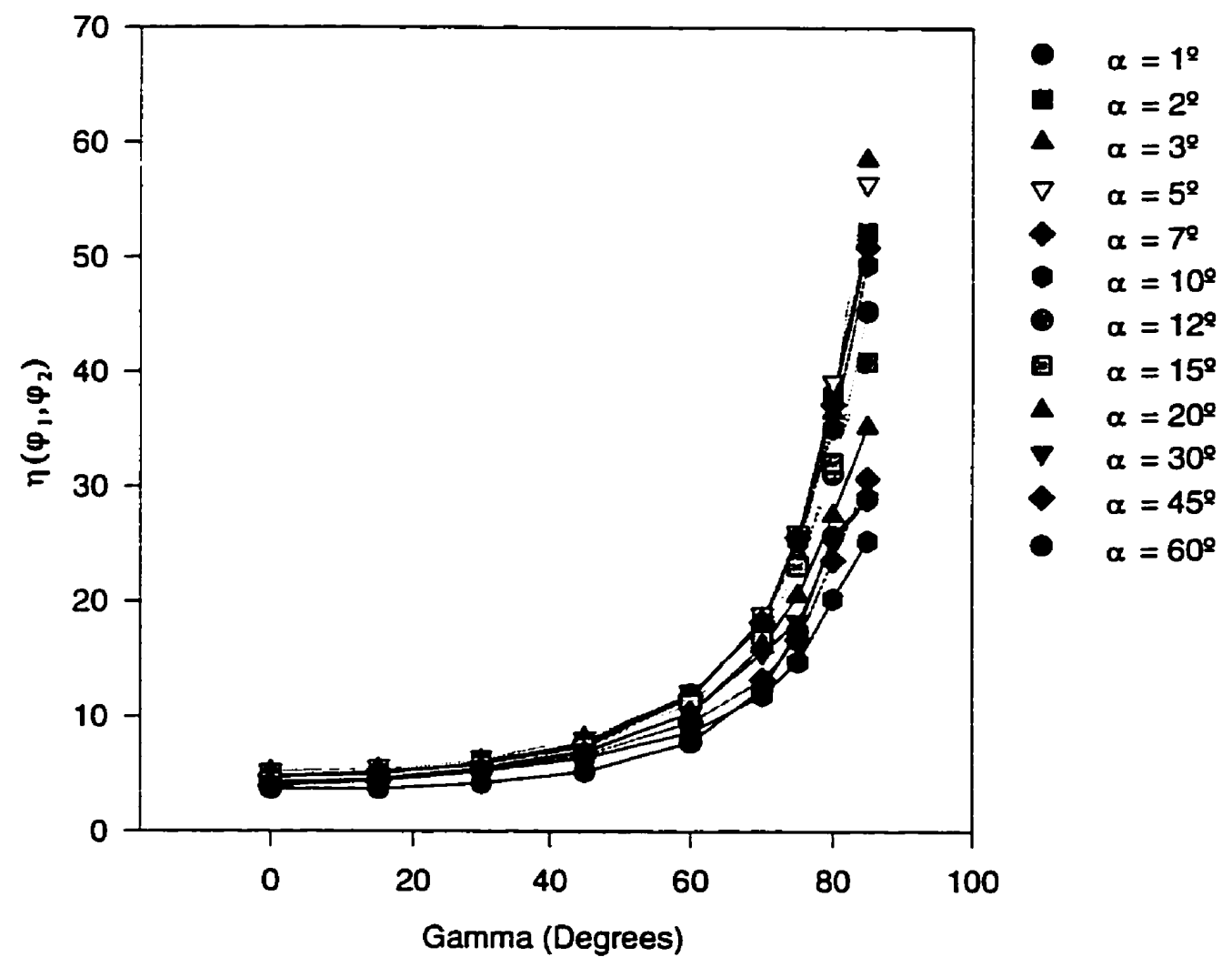

Figure 76 - Diffuse Shadowing Function of R1 Road Class 


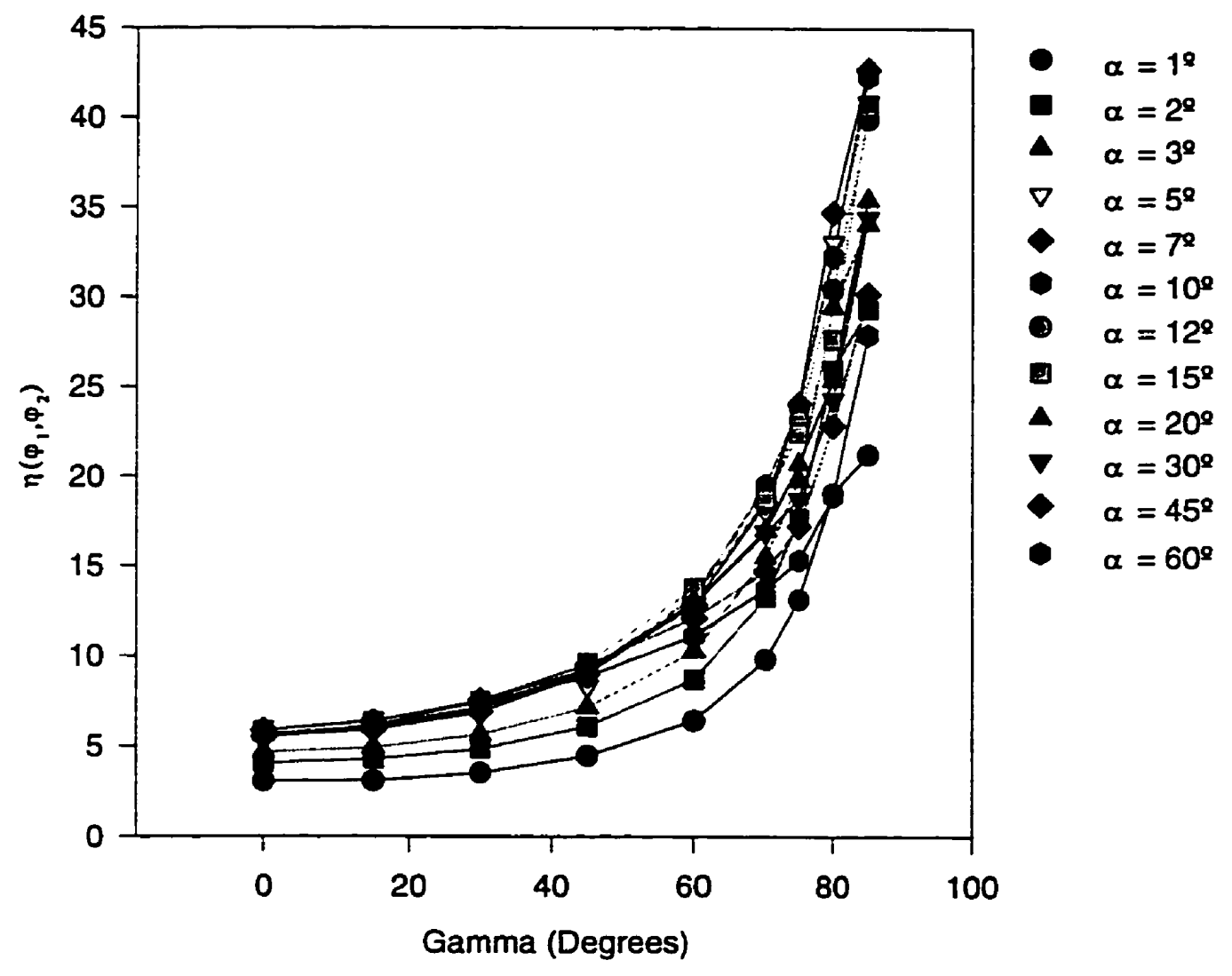

Figure 77 - Diffuse Shadowing Function for R2 Road Class 


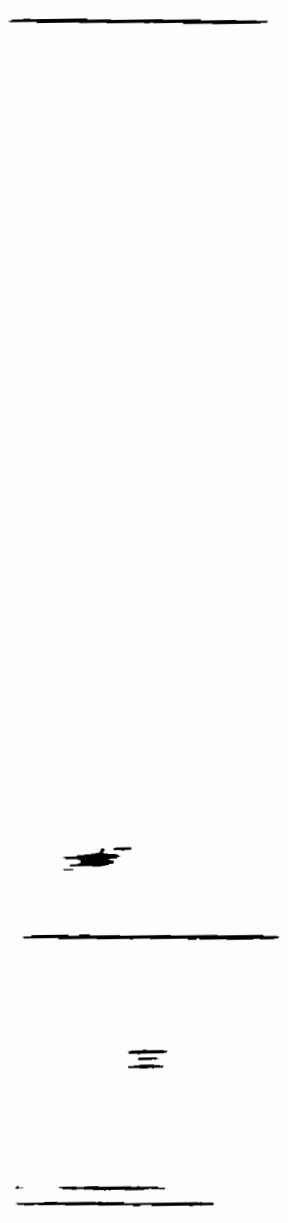




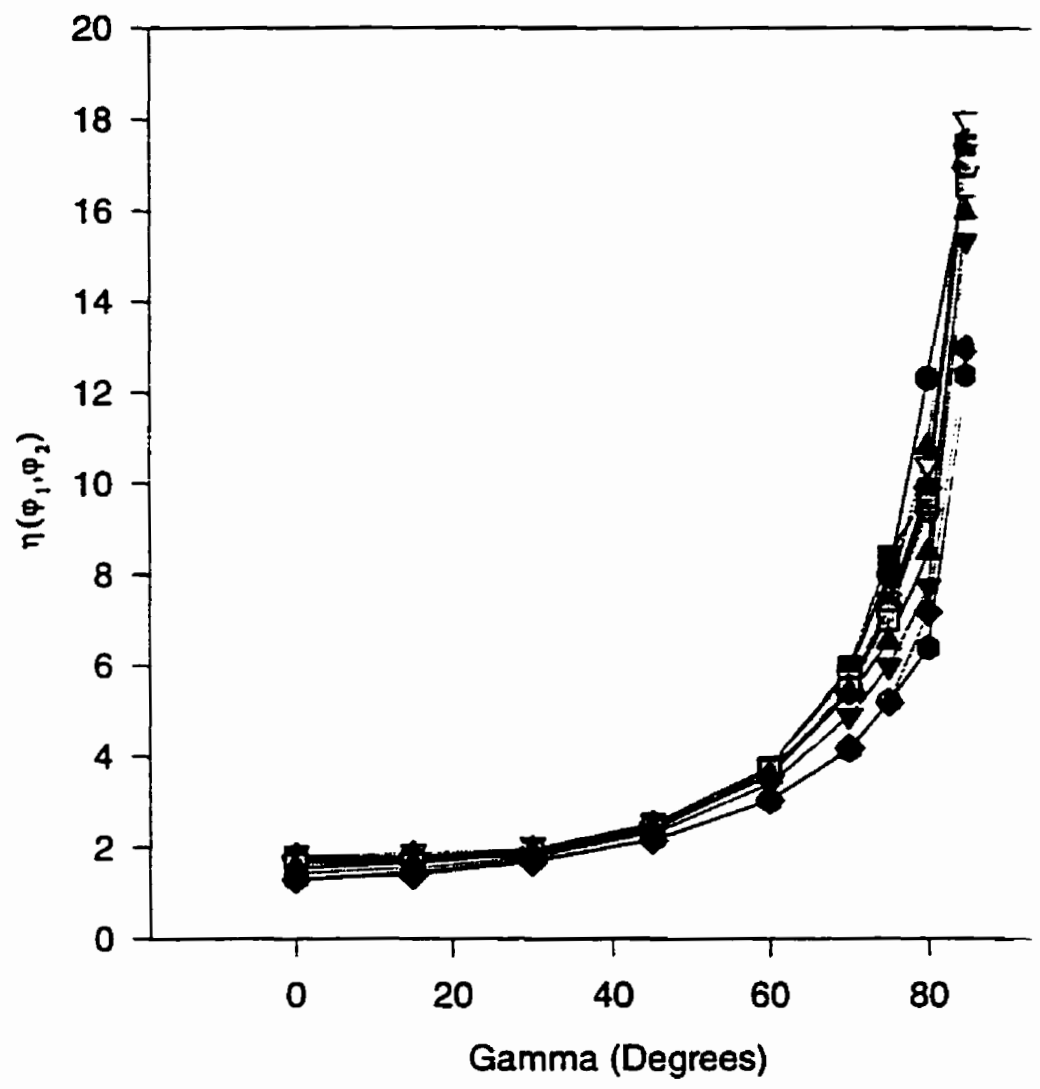

Figure 79 - Diffuse Shadowing Function for R4 Road Class

Mathematical equations were developed for this function usins :-

$$
\eta\left(\varphi_{1}, \varphi_{2}\right)=A+B \cdot e^{\left(\gamma^{c}+D\right)}
$$

This calculation was performed for each sample and the best fir:= were recorded, allowing for the influence of $\alpha$ and pavement st correlation coefficient between the model and the data was extre 
above .99 . The difficulty with continuing this modeling was that there was little correlation between the model parameters and the pavement characteristics. The results of a correlation calculation are shown in Table 25 .

Table 25 - Correlation of Diffuse Model Parameters to Pavement Characteristics

\begin{tabular}{|lrrrrr|}
\hline & S1 & S2 & Q0 & ROUGH & CORR DIST \\
A & -0.488 & -0.487 & -0.399 & 0.116 & 0.575 \\
B & 0.410 & 0.441 & 0.084 & -0.121 & -0.219 \\
C & 0.215 & 0.303 & -0.115 & -0.158 & -0.203 \\
D & -0.389 & -0.474 & -0.039 & 0.188 & 0.272 \\
\hline
\end{tabular}

At this point, the modeling of the diffuse portion was stopped as correlation to the pavement characteristics was required for the data and no relationship of the model to the data could be found.

The specular portion of the reflection was calculated using the measured reflection value minus the diffuse portion as determined above. This calculation was performed with the entire data set. The results for the four R-Classes are shown in Figures 80 to 82 for $\alpha, \beta$ and $\gamma$ respectively. 


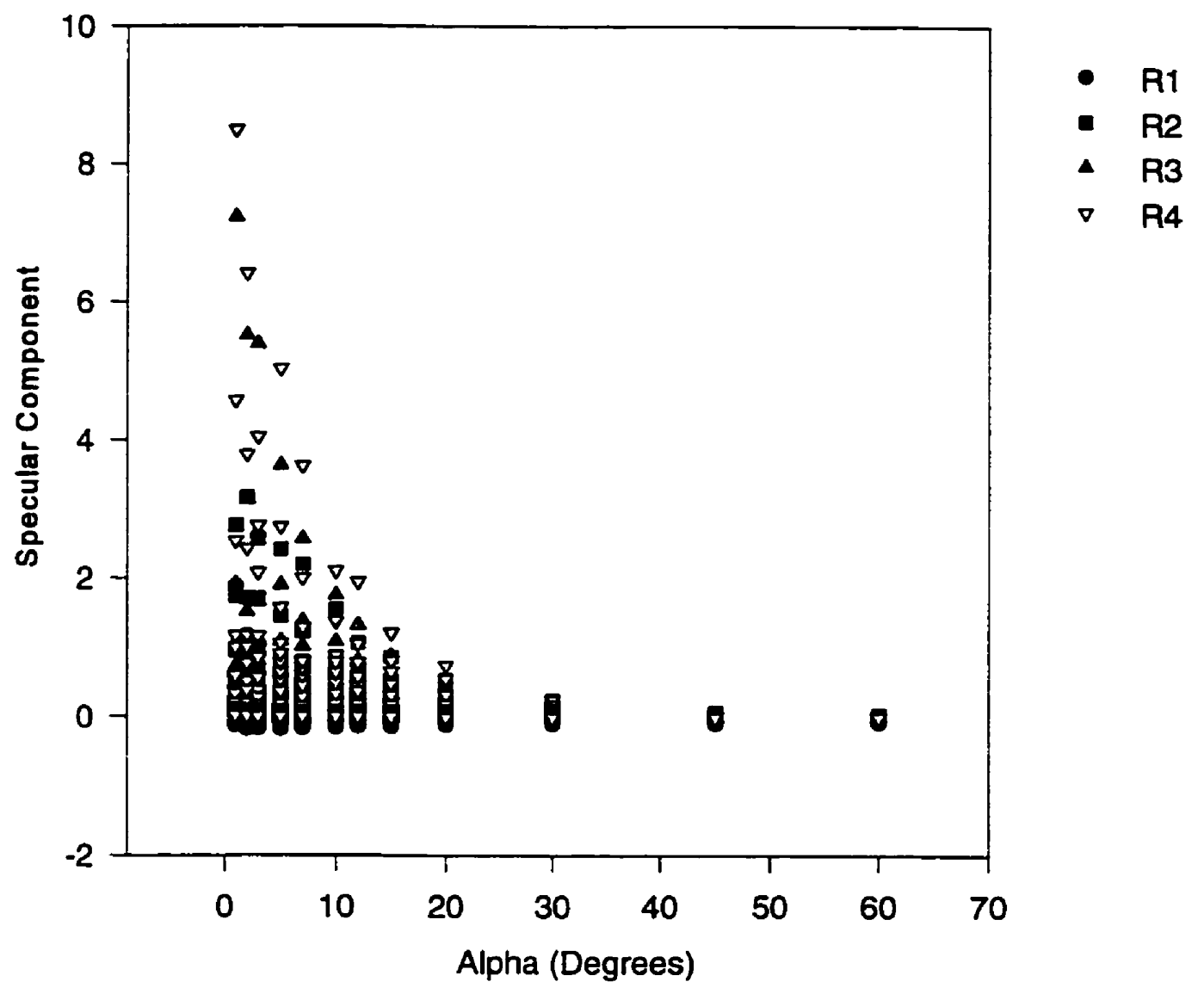

Figure 80 - Relationship of the Specular Component of Reflection to $\alpha$ for the 4 RClasses 


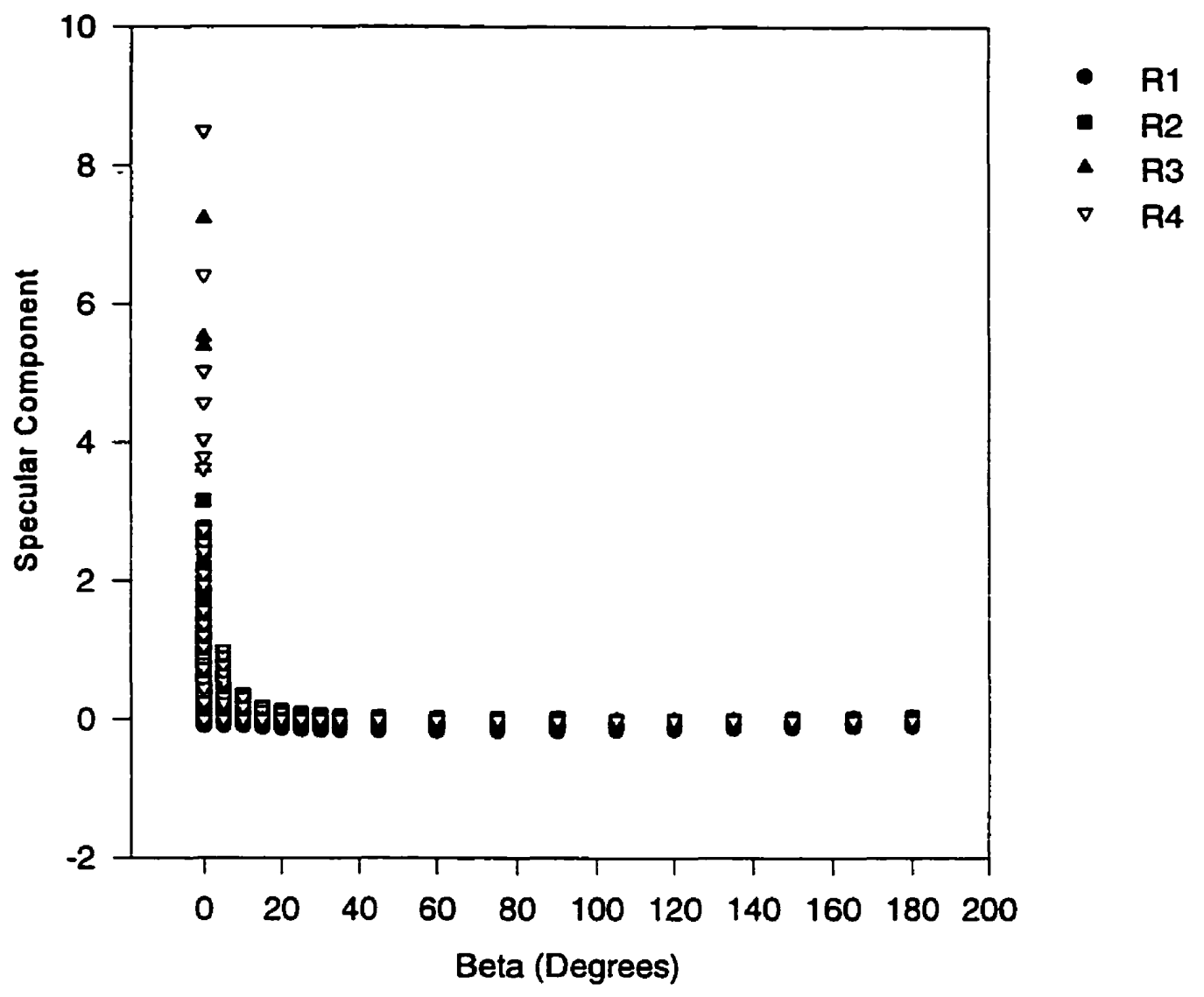

Figure 81 - Relationship of the Specular Component of Reflection to $\beta$ for the 4 RClasses 


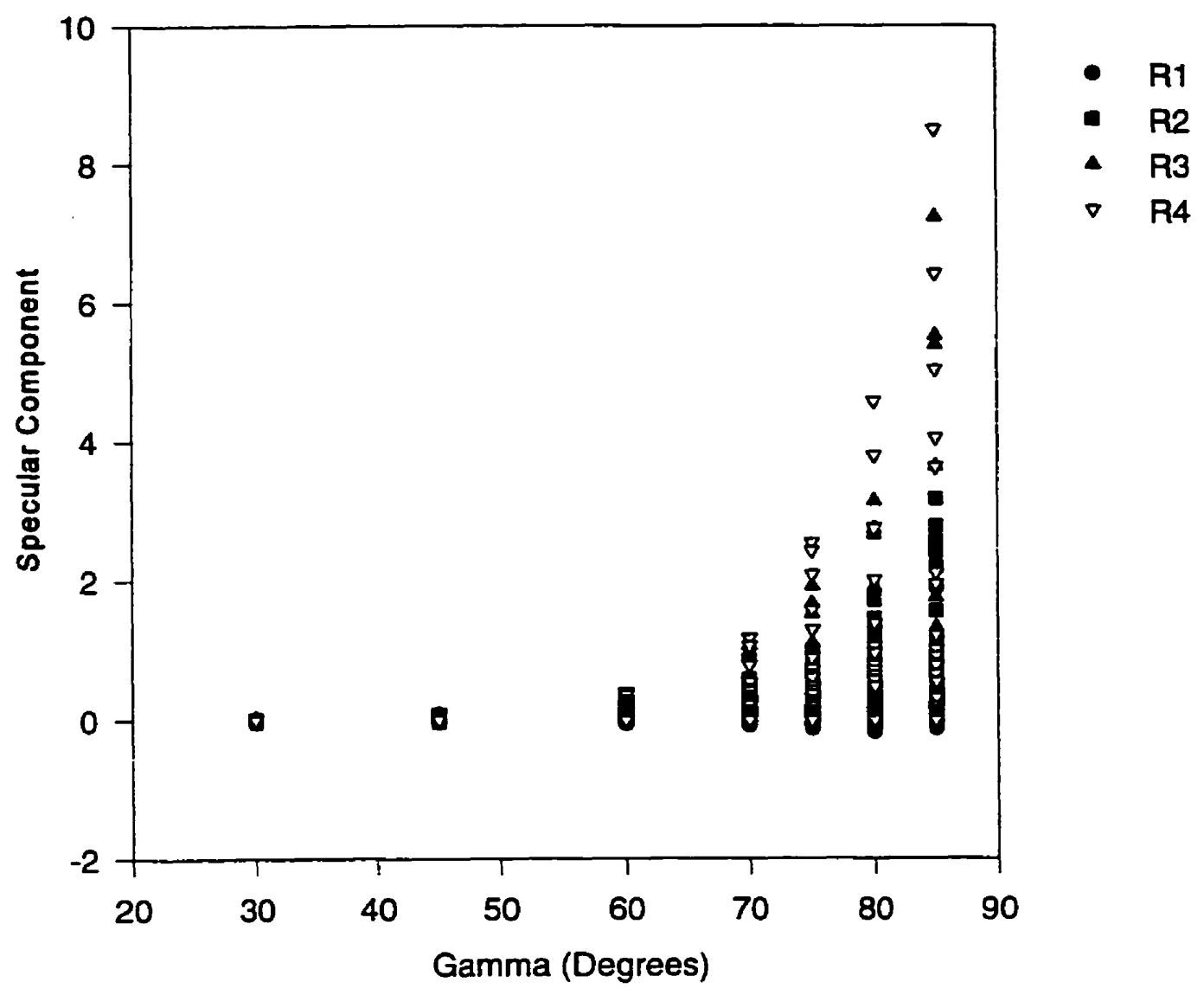

Figure 82 - Relationship of the Specular Component of Reflection to $\gamma$ for the 4 RClasses

The development of the model by Vermeulen required the change in angles from the $\alpha, \beta$ and $\gamma$ notation to $\varepsilon$ and $i$. The specular portion of the reflection for the angles $i$ and $\varepsilon$ is shown in Figures 83 and 84. 


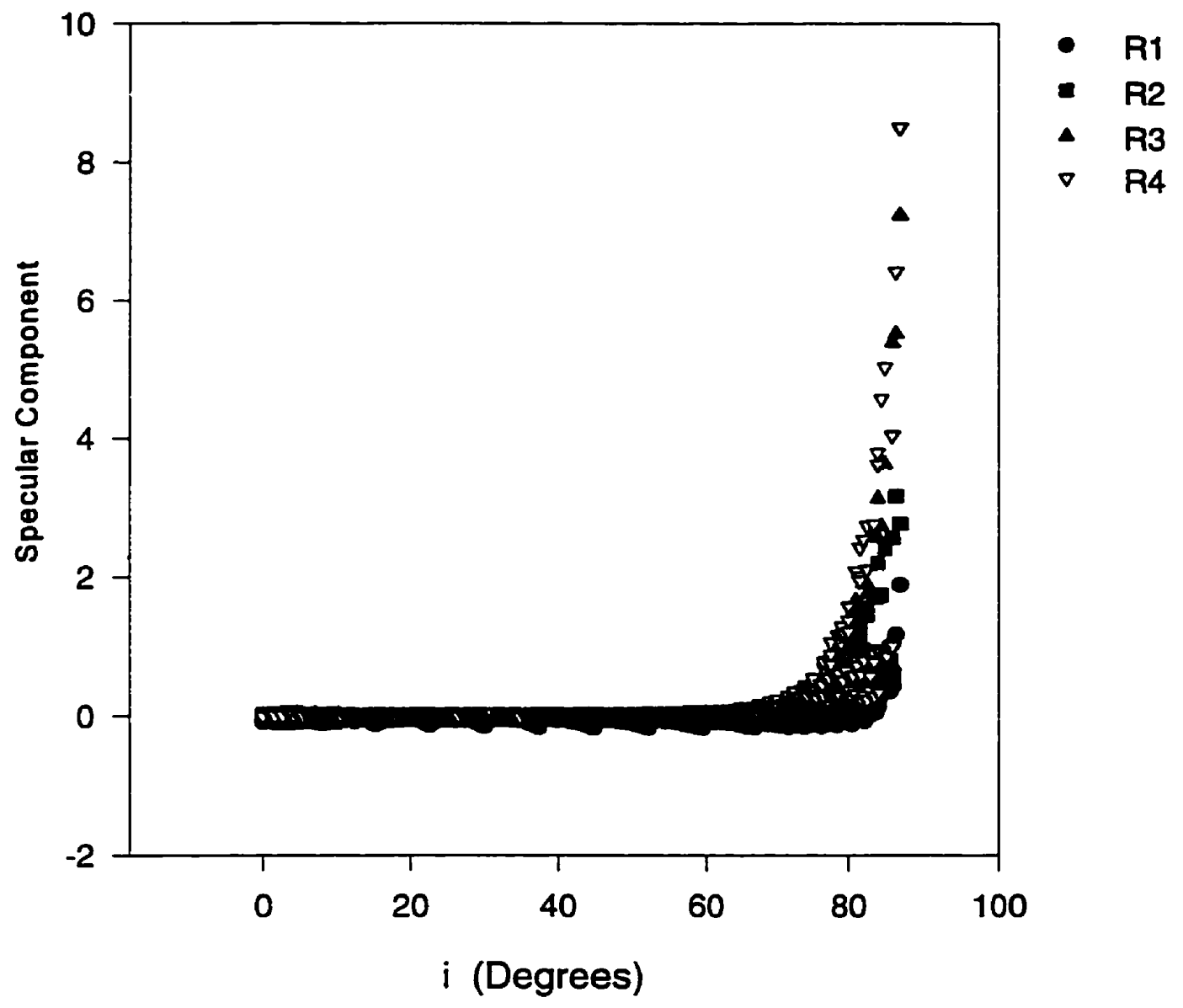

Figure 83 - Relationship of the Specular Component of Reflection to i 


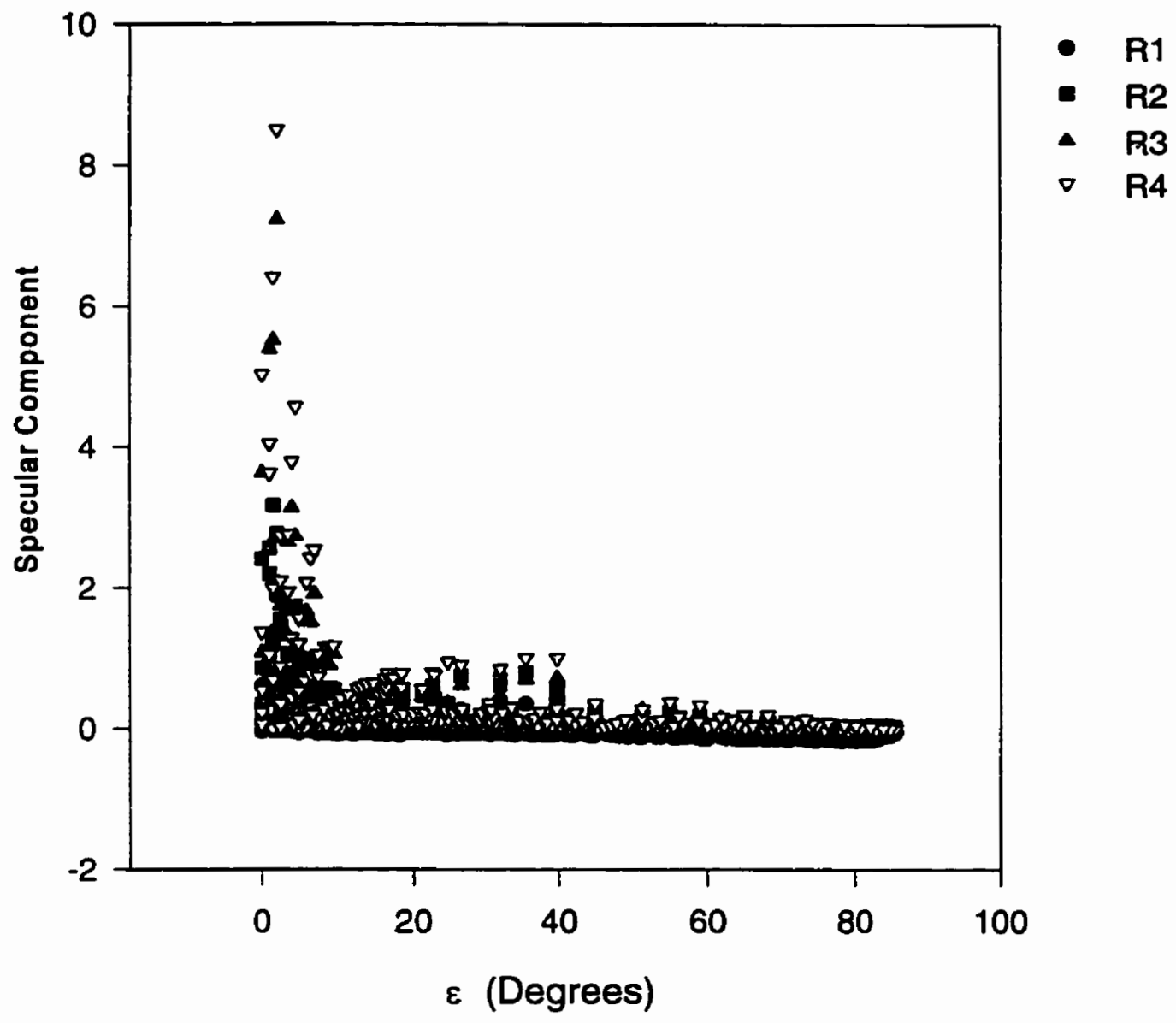

Figure 84 - Relationship of the Specular Component of Reflection to $\varepsilon$

The functions presented in Figures 80 to 84 relate very closely to the expectation.

According to the comparison to Lambertian reflection, there is a specular peak at high $\gamma$, low $\alpha$, and low $\beta$. This is representative of the geometry where the light source is directly opposite the detector and very close to the angle of the surface. Changing the angles to $\varepsilon$ and i does not seem to improve the data analysis rather it seems to add some variability. 
At this point, the modeling according to Vermeulen now required the separation of the specular shadowing function and a function which describes the number of facets which are oriented properly for reflection. There was no way to separate these functions with the data set available to us. Here, again, our knowledge of the pavement surface and the character of the pavement recipe is lacking for continuation of the model development.

\subsection{Physical Optics Model}

The second model considered for this data set is a system which uses the nature of the surface and the diffraction of light as the basis of the calculation. Diffraction is defined as the deviation of electromagnetic waves from rectilinear paths of propagation due to interference with obstacles, edges and surfaces (Nieto-Vesperinas [1991]). Diffraction and scatter are the processes which light undergoes when it is reflecting from surfaces. As discussed previously, as a surface becomes rougher, the reflection will no longer be specular but diffuse, scattering in all directions. The calculation of the resulting reflection field can be performed using a model of the surface which describes its roughness characteristics. This model was originally defined for the transmission and reflection of radio waves.

\subsubsection{Model Development}

For this calculation, the first question that was dealt with was the definition of a rough and a smooth surface. Beckmann and Spizichinno [1963] defined these surfaces using the Rayleigh criterion which considers two rays incident on a surface with a roughness of 
$h$ and an angle of incidence of $\gamma$. This situation is shown in Figure 85.

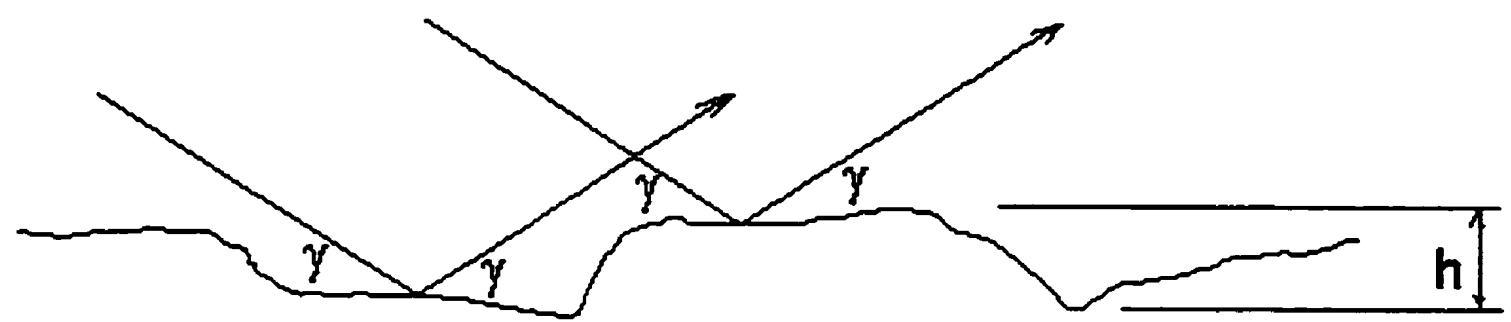

Figure 85 - Rayleigh Criterion Geometry

The phase difference in the reflected light rays is determined by the difference in the distance the light rays must travel. This difference is:

$$
\Delta d=2 \cdot h \cdot \sin (\gamma)
$$

Therefore the phase difference is:

$$
\Delta \varphi=\frac{2 \cdot \pi}{\lambda} \cdot \Delta d=\frac{4 \cdot \pi \cdot h}{\lambda} \cdot \sin (\gamma)
$$

When the phase difference is close to 0 , the light reflects specularly. If the phase difference is close to $\pi$, the light rays will scatter and cancel each other. These extremes of the criterion are characteristics of smooth and rough surfaces respectively. By choosing the halfway point, the Rayleigh criterion is found. This means that a surface is considered rough if: 


$$
h>\frac{\lambda}{8 \cdot \sin (\gamma)}
$$

For the visible light spectrum, a surface height change has to be very small in order for the surface to be considered smooth. The extreme limits of the visible light spectrum are $350 \mathrm{~nm}$ and $800 \mathrm{~nm}$. Outside of these wavelength limits, radiation is not perceived by the human eye. In Equation 57, the maximum roughness limit would be calculated with 800 $\mathrm{nm}$ and for our experiment $\gamma=85^{\circ}$. (Note: The Rayleigh criterion defines $\gamma$ from horizontal rather than from the normal.) This limit is calculated as $1147.4 \mathrm{~nm}$. The lowest mean surface roughness measured on these samples was $0.147 \mathrm{~mm}$ which means that all of the samples in this experiment must be considered rough surfaces.

\subsubsection{General Kirchoff Solution}

Beckmann and Spizzichino formulated the calculations of the BRDF in terms of the roughness of the surface of the material. This calculation uses the Kirchoff approximation of diffraction. The derivation defines the surface roughness as a function, $\zeta(x, y)$, in terms of the mean deviation of the height of the surface from the mean $x y$ plane where $x$ and $y$ are in the plane parallel to the surface. This surface has an incident light wave on it at a point, p. The resulting scattered field is received at a point, P. The incident light has a direction vector, $\mathbf{k}_{1}$, which is given by $\beta$ and $\gamma$. The light received at point $\mathrm{P}$ has a direction vector, $\mathbf{k}_{\mathbf{2}}$, defined by $90-\alpha$ and $\phi$. (Note: $90-\alpha$ is used in the calculation in that the mathematical development was performed using angles to the normal of the surface and not to the surface plane.) The nature of the incident light wave 
and the received field are defined as $\mathbf{E}_{1}$ and $\mathbf{E}_{2}$ respectively. The field $\mathbf{E}_{1}$ is:

$$
\vec{E}_{1}=E_{0} \cdot e^{i(\vec{k} \cdot \vec{r}-\omega \cdot t)}
$$

where $\omega$ is the angular frequency of the incident light and the position vector in time is:

$$
\vec{r}=x \vec{x}_{0}+y \vec{y}_{0}+z \vec{z}_{0}
$$

Beckmann and Spizzichino showed that the intensity of the incident light is equal to the square of the scalar amplitude of the incident light wave $E_{0}$.

The Kirchoff approximation of diffraction yields the resulting field defined by the Helmholtz integral:

$$
E_{2}(P)=\frac{1}{4 \pi} \iint_{S}\left(E \frac{\partial \Psi}{\partial n}-\frac{\partial E}{\partial n}\right)
$$

where

$$
\Psi=\frac{e^{i k_{2} R_{2}}}{R_{2}}
$$

$R_{2}$ is the distance from the incident point $\mathrm{p}$ to the receiving point, $\mathrm{P} . \mathrm{n}$ is the normal to the surface and $k_{2}$ is the scalar magnitude of the propagation vector, $\mathbf{k}_{2}$. 
Beckman introduced the scattering coefficient $(\rho)$ which is a dimensionless relationship of the scattered field $\mathbf{E}_{2}$ to the field which would result from a smooth perfectly reflecting plane received in the specular direction defined as $\mathbf{E}_{20}$.

$$
\rho=\frac{E_{2}}{E_{20}}
$$

Beckmann then developed the equation for $\rho$ using the Helmholtz integral and some boundary conditions as:

$$
\rho=\frac{F}{A} \iint_{A} e^{i \bar{v} \cdot \bar{r}} d x d y
$$

where

$$
F=\frac{1+\cos (\gamma) \cos (90-\alpha)-\sin (\gamma) \sin (90-\alpha) \cos (\beta)}{\cos (\gamma) \cdot(\cos (\gamma)+\cos (90-\alpha))}
$$

In Equation 63, A represents the area of the surface in the $x y$ plane and the vector $v$ is:

$$
\begin{aligned}
\vec{v} & =\vec{k}_{1}-\vec{k}_{2} \\
& =k\left[(\sin (\gamma)-\sin (90-\alpha) \cdot \cos (\beta)) \vec{x}_{0}-(\sin (90-\alpha) \cdot \sin (\beta)) \vec{y}_{0}-(\cos (\gamma)+\cos (90-\alpha)) \vec{z}_{0}\right]
\end{aligned}
$$

The position vector, $\mathbf{r}$, describes the rough surface.

$$
\vec{r}=x \vec{x}_{0}+y \vec{y}_{0}+\zeta(x, y) \vec{z}_{0}
$$


It is noteworthy that this equation assumes that the surface is perfectly reflecting which means that there is no absorption of light by the surface.

\subsubsection{Normal Distribution Solution}

Having developed the general solution to the problem of light scatter from rough surfaces, the problem was reduce to the evaluation of the field for an unknown $\zeta(x, y)$. For surfaces which have been machined or treated with special forms, an equation for the surface variation can be found. For a rough surface, such as pavement, an equation would be very difficult to develop. This surface is a random rough surface and must be treated in a statistical manner.

Beckmann found a solution using the probability density function which yielded a surface height based on the distance between two points. If the function $\zeta(x, y)$ is considered to be a random variable producing surface heights, the joint probability function is $p\left(z_{1}, z_{2}\right)$, given that $z_{1}$ and $z_{2}$ are the surface heights at points $\left(x_{1}, y_{1}\right)$ and $\left(x_{2}, y_{2}\right)$ respectively. By placing the Fourier transform of the probability density function into the general solution outlined above and integrating, Beckmann found the solution of the mean scattered field in any direction for a general case where the probability density is known. This solution is:

$$
\langle\rho\rangle=\frac{F}{A} \cdot \chi\left(V_{z}\right) \cdot \rho_{0}
$$

where $\chi\left(V_{2}\right)$ is the Fourier transform of the probability density, $\mathrm{F}$ and $\mathrm{A}$ are as previously 
defined, and $\rho_{0}$ is:

$$
\rho_{0}=\frac{\sin \left(v_{x}\right) X}{v_{x} X} \cdot \frac{\sin \left(v_{y}\right) Y}{v_{y} Y}
$$

where $X$ and $Y$ are the limits of the integration area $(A=X Y)$.

Beckmann then introduced the normal probability distribution into the calculation. The function $\zeta(\mathrm{x}, \mathrm{y})$ is considered to be normally distributed with a mean $=0$, a standard deviation of $\sigma$ and an auto-covariance $C(\tau)$, where:

$$
\tau=\sqrt{\left(x_{2}-x_{1}\right)^{2} \cdot\left(y_{2}-y_{1}\right)^{2}}
$$

and the auto-covariance is Guassian,

$$
C(\tau)=e^{\frac{-|\tau|}{\tau}}
$$

The variable $T$, is the correlation distance as measured and defined in Chapter 6. In general, the Kirchoff approximation requires that the correlation distance, $T$, be much larger than the wavelength $(T>>\lambda)$.

Beckman used this definition to develop the solution for the mean square of the scattering coefficient which is proportional to the mean scattered field. This solution is: 


$$
\left\langle\rho \rho^{\circ}\right\rangle=\frac{\pi F^{2} T^{2}}{A g} \exp \left(-\frac{v_{x y}^{2} T^{2}}{4 g}\right)
$$

where $\rho^{*}$ is the complex conjugate to $\rho$, and:

$$
\sqrt{g}=v_{z} \sigma=\frac{2 \pi \sigma}{\lambda}(\cos (\gamma)+\cos (90-\alpha))
$$

for values of $g>>1$. The variable $g$ is the ratio of the standard deviation of the surface roughness to the wavelength and it represents the importance of the specular reflection in the solution. For g close to zero, specular reflection is the major component of the solution, where as for $\mathrm{g} \gg>1$, the diffuse reflection is the most important characteristic.

The value is related to the intensity of the incident and reflected light because by definition:

$$
\left\langle\rho \rho^{\cdot}\right\rangle=\frac{\left\langle\left|E_{2}\right|^{2}\right\rangle}{E_{20}^{2}}=\frac{I_{r}}{I_{i}}
$$

where $L_{i}$ is the intensity of the reflected field and $\mathrm{I}_{j}$ is the intensity of the incident field. A more complete development of this formulation is found in Beckmann and Spizzichino.

This solution allows the use of the standard deviation of the surface height and the correlation distance to estimate the mean power of the scattered field and the intensity of the reflected field in terms of the incident field. This value calculated at different angles 
of incidence and reflection should then be proportional to the value of the reflection factor $q(\alpha, \gamma, \beta)$.

This solution development does not account for any effects of polarization. These, however, should be minimized since the light source being used for the reflection data is incoherent.

The solution also assumes a perfectly reflecting surface. This factor can be accounted for using a factor which scales the overall reflection coefficient. This factor should be related to the $q_{0}$ of the surface.

The other difficulty of this calculation methodology is shadowing across the surface of the road sample. This shadowing function is similar to that in the facet model. Numerically, it is unlikely that this shadowing function would be the same as that developed for the facet model. The shadowing function in this model would scale the integration area to include only the illuminated surfaces.

\subsubsection{Model Application}

A computer program was developed to implement the physical optics method as described above. The program relies on the input parameters of surface roughness, the correlation distance, sample area, test wavelength, and the three angles, $\alpha, \beta$ and $\gamma$. The 
program output is the reflectivity factor $\left\langle\rho \rho^{*}>\right.$ for $\alpha, \beta$, and $\gamma$. The specularity factor, $g$, described in Equation 72, is also output from the program.

The area used in the program is determined based on the surface area of the sample scaled by the sine of the observation angle. As $\alpha$ decreases, the projected area of the sample surface also decreases by $\sin (\alpha)$.

The results of this model calculation for sample Ontario L7-4 are shown in Figures 86 through 88 for $\alpha, \beta$ and $\gamma$ respectively. The data presented here are generally representative of the results from the calculations for all of the samples. 


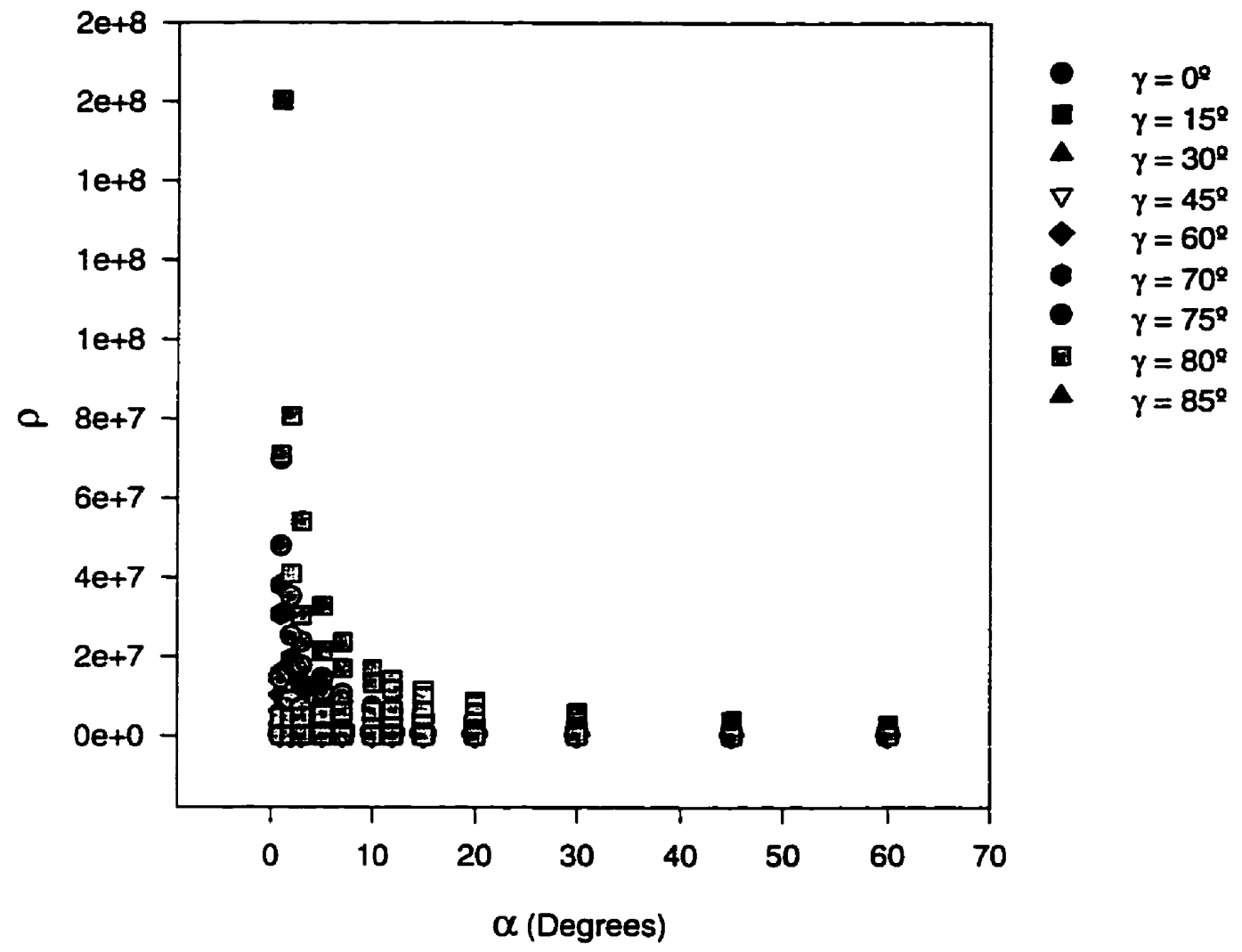

Figure 86 - Calculated Results for Physical Optics Model in terms of $\alpha$ for Sample Ontario L7-4 


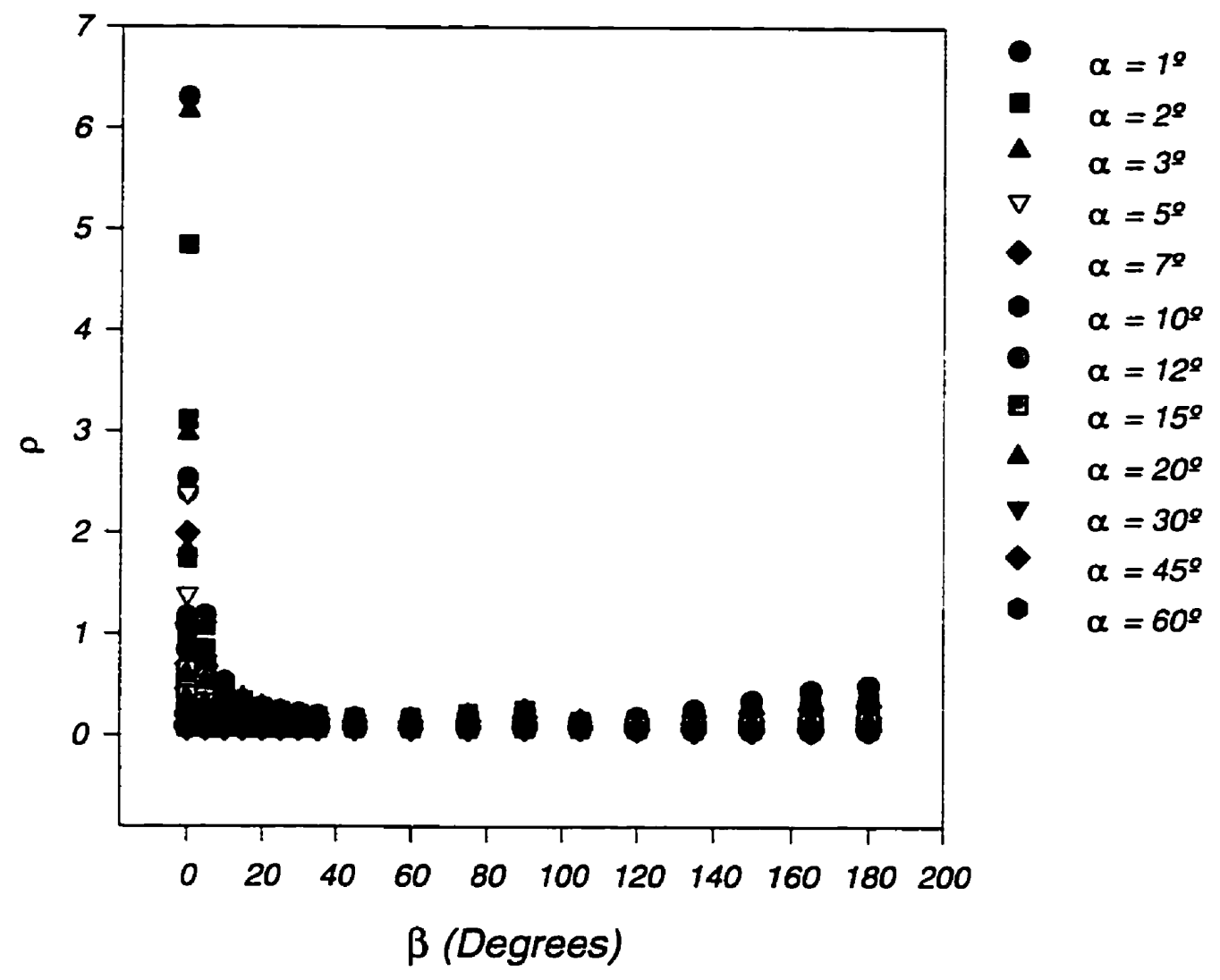

Figure 87 - Calculated Results for Physical Optics Model in terms of $\beta$ for Sample Ontario L7-4 


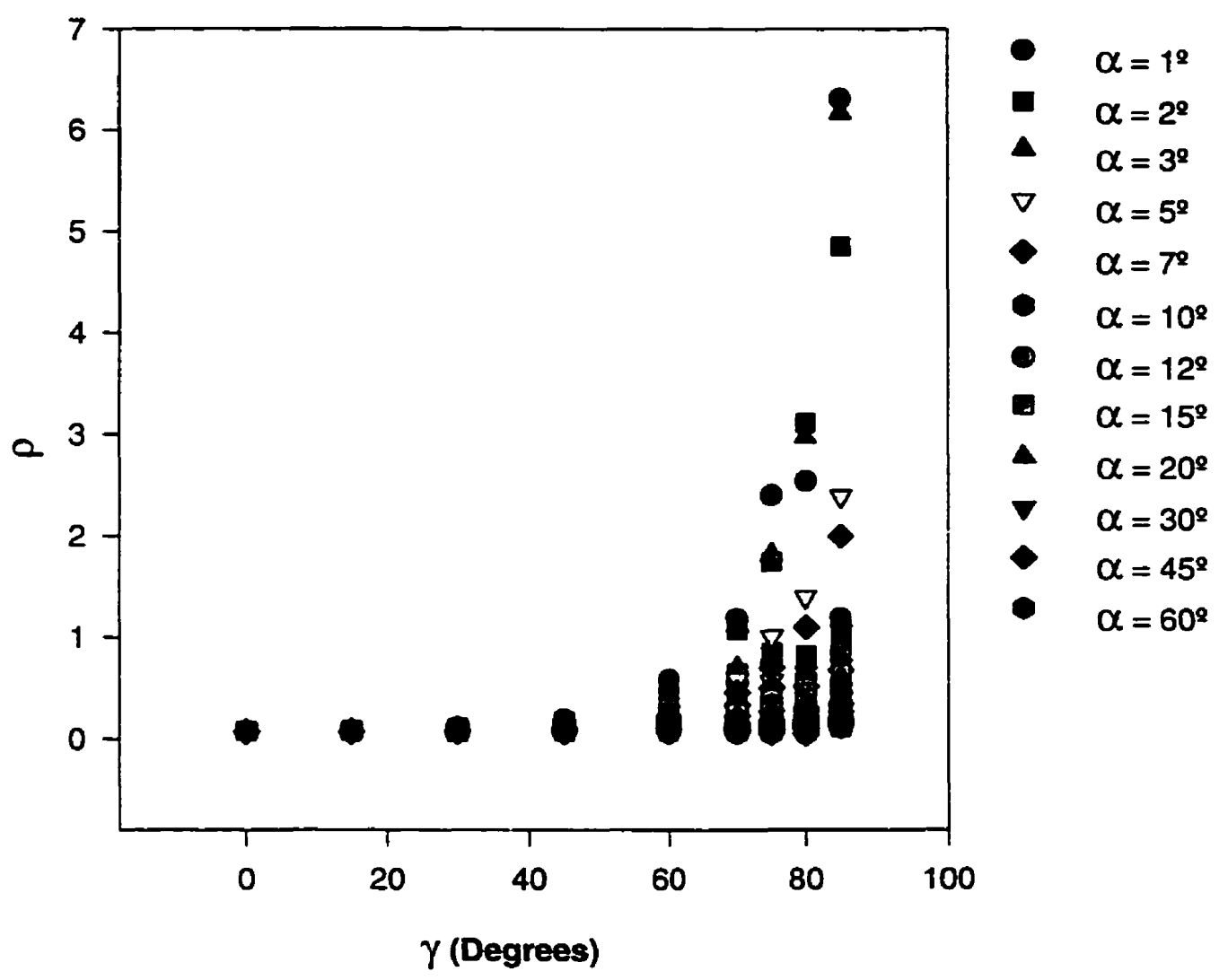

Figure 88 - Calculated Results for Physical Optics Model in terms of $\gamma$ for Sample Ontario L7-4

These figures show all of the data points calculated for each sample. Many of the points calculated are coincident on the graphs.

These calculations resulted in a very high specularity factor. This means that the nature of the reflection is highly diffuse which has already been shown with the reflection data. The data shows a similiarity in the shape of the calculated curves to those of the reflection data. The calculated results were compared to those of the reflectivity 
experiment. This comparison is shown in Figure 89 for sample Ontario L7-4.

The model calculates many points where the reflection results equal zero. This means that the model is predicting that no reflection will occur at this point. We know from the reflection experiment that there are points of low reflectivity but very few of zero reflection. This lack of sensitivity of the model for low reflection values and the general correlation of the model to the high reflection values, indicate that the physical optics model is basically calculating the specular reflection and not considering diffuse reflection. 


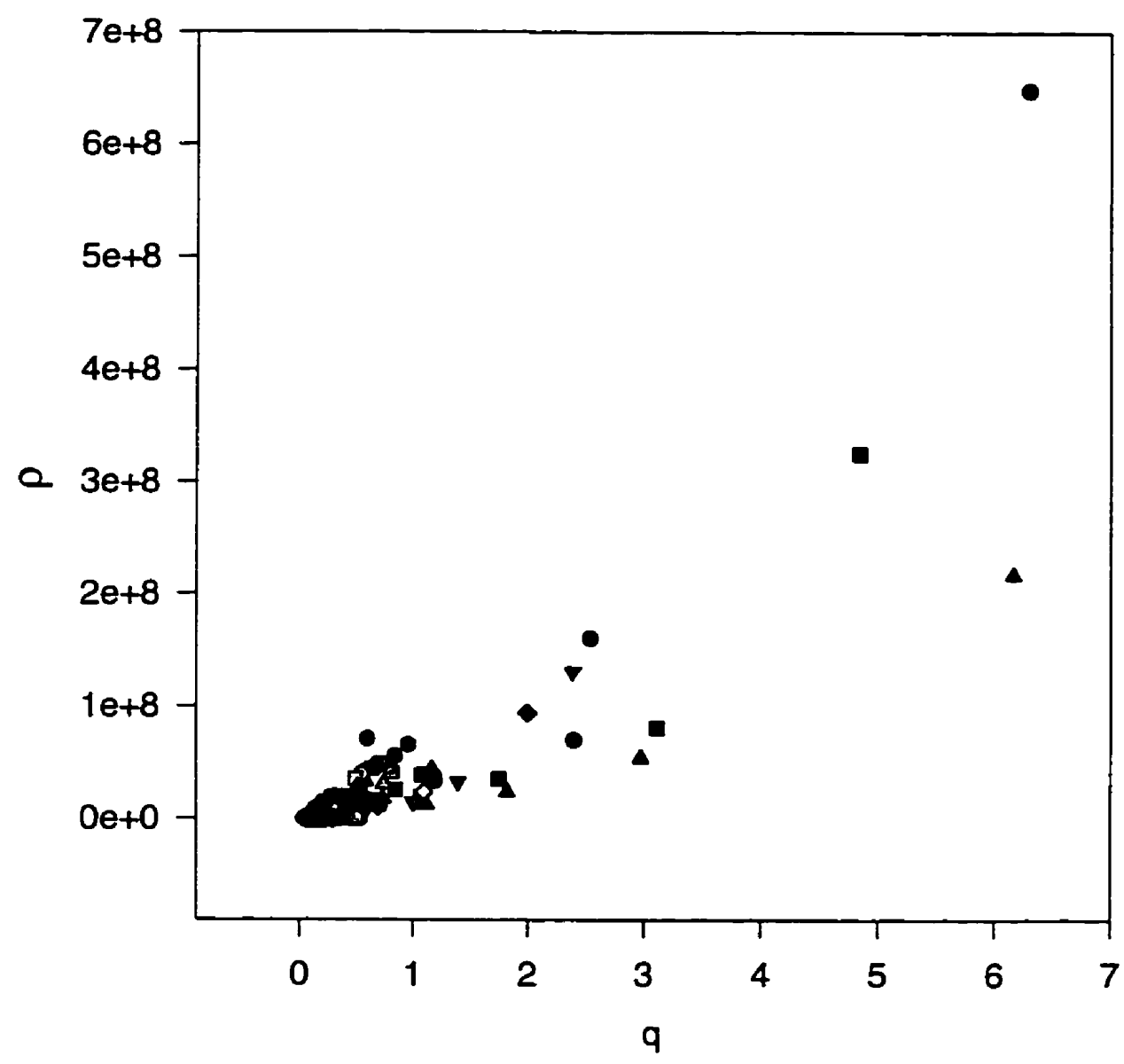

Figure 89 - Comparison of Reflection Results and Calculated Physical Optics Model for Sample Ontario L7-4

The relationship of the model results to the reflectivity results was established using a linear regression model. This regression was performed for each sample individually using Equation 74. The regression results are shown in Table 26.

$$
q(\alpha, \beta, \gamma)=A \cdot \rho(\alpha, \beta, \gamma)+B
$$


Table 26 - Linear Regression Results for the Physical Optics Model

\begin{tabular}{||l|r|r|r|}
\hline \multicolumn{1}{|c|}{ Sample Name } & \multicolumn{1}{c|}{$\mathrm{r}^{2}$} & \multicolumn{1}{c|}{ Slope } & \multicolumn{1}{c|}{ Intercept } \\
\hline Alberta \#1 & 0.893 & $4.74 \mathrm{E}-09$ & 0.1175 \\
\hline British Columbia N4 Hwy 19 & 0.70781 & $5.46 \mathrm{E}-10$ & 0.0777 \\
\hline Manitoba PR 200 1BWP & 0.69326 & $2.35 \mathrm{E}-09$ & 0.2105 \\
\hline New Brunswick Rt2 \#5 & 0.69197 & $1.55 \mathrm{E}-09$ & 0.0905 \\
\hline Nova Scotia \#11 & 0.820021 & $2.53 \mathrm{E}-09$ & 0.0760 \\
\hline Nova Scotia \#15 & 0.58879 & $5.52 \mathrm{E}-09$ & 0.0766 \\
\hline Ontario 19-14 & 0.62989 & $1.04 \mathrm{E}-09$ & 0.0820 \\
\hline Ontario 19-17 & 0.71899 & $1.66 \mathrm{E}-08$ & 0.0873 \\
\hline Ontario 19-3 & 0.66567 & $1.48 \mathrm{E}-09$ & 0.1000 \\
\hline Ontario 19-7 & 0.75037 & $3.98 \mathrm{E}-09$ & 0.0877 \\
\hline Ontario L7-2 & 0.55375 & $8.32 \mathrm{E}-09$ & 0.1129 \\
\hline Ontario L7-3 & 0.76533 & $3.19 \mathrm{E}-08$ & 0.1109 \\
\hline Ontario L7-4 & 0.7944 & $1.34 \mathrm{E}-08$ & 0.1130 \\
\hline PEI \#2-K & 0.72813 & $3.46 \mathrm{E}-09$ & 0.1419 \\
\hline Quebec \#4 & 0.67036 & $2.46 \mathrm{E}-10$ & 0.1599 \\
\hline Quebec \#6 & 0.54476 & $8.06 \mathrm{E}-11$ & 0.2229 \\
\hline Saskatchewan WL \#10 & 0.8832 & $2.15 \mathrm{E}-09$ & 0.0970 \\
\hline Saskatchewan WL \#32 & 0.50495 & $2.95 \mathrm{E}-09$ & 0.1093 \\
\hline Saskatchewan WL \#37 & 0.67471 & $8.40 \mathrm{E}-10$ & 0.0612 \\
\hline Saskatchewan WL \#8 & 0.66033 & $7.00 \mathrm{E}-10$ & 0.0643 \\
\hline \hline
\end{tabular}

The slope in this linear regression is representative of a multiplier to account for the reflectivity of the surface and the shadowing on the surface. The intercept represents a shift in the data similar to a diffuse nature overlayed with specular reflection. Based on this, a correlation of the slope or the intercept should be evident with the characteristics of $\mathrm{S} 1$, roughness or $\mathrm{q}_{0}$ in the pavement sample. This correlation was performed and is shown in Table 27. 
Table 27 - Correlation Coefficients of Linear Regression Parameters to Surface Characteristics

\begin{tabular}{|c|c|c|c|c|c|c|}
\hline $\begin{array}{l}\text { Slope } \\
\text { Intercept }\end{array}$ & $\begin{array}{r}\text { S1 Corr } \\
0.339 \\
-0.392\end{array}$ & $\begin{array}{r}\text { S2 Corr } \\
0.383 \\
-0.494\end{array}$ & Qo & $\begin{array}{l}- \text { Corr } \\
0.367 \\
0.233\end{array}$ & $\begin{array}{r}\text { Ave Roug } \\
0.296 \\
-0.091\end{array}$ & $\begin{array}{r}\text { Ave Corr } \\
-0.286 \\
0.564\end{array}$ \\
\hline
\end{tabular}

There is very low correlation evident in these factors to the pavement characteristics which leads to difficulties in the model. In some cases, the differences in the slope between samples were as much as an order of magnitude or higher. Changes in the reflection data between samples were not as significant as those in the calculated model.

The physical optics model has no allowance for micro roughness; only macro roughness. In has been previously indicated the specular reflection is a result of the macro roughness whereas the diffuse reflection was a result of the micro roughness. The results are confirmed in the modeling of the data. The physical optics method, which is based on macro roughness, is most closely related to the specular portion of the reflection.

\subsection{Discussion}

In the two models considered, the reflection data can be calculated by the methods used, but a final and complete model was not able to be developed. In order to fully develop an analytical model for the reflection properties of pavement, extensive knowledge of the pavement surface is required. The roughness and the reflectance of the individual facets of the pavement surface must be determined in order to relate this characteristic to the reflection results. 
Although the justification for developing a model was ease of use, the sacrifices in the accuracy of the reflection data may be too great for the precision required of the resulting luminance calculations. In the application of the Vermeulen model, the small deviations in the reflection were lost in the model development, overshadowed by the magnitude of the other data in the calculation. Similar to this, the White model stated a $400 \%$ deviation for high values of $\gamma$ and $\beta$. The value to the user of any analytical model is the accuracy of the results. If a model is able to be developed for the reflection properties of pavement, careful consideration must be given to the final results. Although an empirical system is by nature cumbersome, the accuracy of the result is not sacrificed to fit a generalized form of equation.

\subsection{Conclusions}

The results of the reflection experiment show a close relationship in the data to Lambertian reflection. A specular component is also very evident. The models used to describe the reflection of light from a surface, the facet model developed by Vermuelen and a physical optics models described by Beckmann and Spizzichino, both use mathematical methods to seperately describe the specular and diffuse components.

Although the model development and application show very promising results, a complete model was not able to be developed. More complete knowledge of the pavement sample is required. Similarly, other experimental methods can be considered to more fully develop the specular and diffuse nature of the pavement reflection. The 
nature of the macro and the micro roughness of the sample also needs to be more fully developed and implemented in the models.

Although an analytical model is used more easily than an empirical model, particular care must be taken in the use of any model developed. The sacrifices in the accuracy of the model will be great at low reflection values, which might prove too great in terms of the calculations being performed. 


\section{Target Visibility}

The influence of the pavement reflection on target visibility is calculated by a computer program which includes the intensity of the light from the street lighting luminaires, the pavement reflection characteristics of the roadway and the geometry of the lighting installation.

The use of such a calculation program has been questioned. The methods used for defining the quality of a lighting design have become increasingly complex as time and research have progressed. The original quality criterion of illuminance was calculated by hand; computer systems were later used to ease the process. With the introduction of the luminance criteria, the calculations became much more complex, requiring computer systems for calculation. The target luminance and the visibility level calculations have added an even greater reliance on computer programs and calculations. As the complexity of the calculation systems has increased, the physical verification of the calculation results has also become increasingly more difficult, if not impossible.

Janoff [1993] presented a comparison of measured luminance and target luminance results to calculated results for the same lighting installation and found little relationship between these values. The best luminance correlation found by Janoff was $7 \%$ for the pavement luminance and $11 \%$ for the target luminance. Janoff also found several locations in which the measured contrast was actually inverse to the calculated contrast. This means that the calculation results showed that the target was brighter than the 
surface, but the measurements showed the target was actually darker than the surface or vice versa. As the visibility level is based on both the background and the target luminance, the composite error is much greater than the error in the individual luminance calculations. These results have also been one of the barriers to the acceptance of the revised RP-8. Several investigations have been undertaken to further verify the calculation results and explain the deviation. Most of these have been performed by the Visibility Task Force (VTF) of the Roadway Lighting Committee of the IESNA. The research undertaken on the reflection properties of pavement surfaces is also based on this requirement for clarification.

In order to fulfill this requirement of the research, a computer program to implement the target luminance with pavement reflection and the visibility level was written based on the calculation method prescribed by IESNA RP-8 [1997]. This program was used to attempt to verify the measurements of the VTF.

\subsection{Visibility Computer Program}

The original program for calculating the luminance of pavement surfaces for street lighting design was written in the late 1960's at the Technical University of Karlsruhe (Adrian and Enzmann [1971]). This program was originally coded on computer cards and took several hours to perform a design calculation. This program, written in Fortran, was published as part of the CIE Guide 30.2 [1982] and was given the name LUCIE, which stands for Background Luminance (Lu) according to the CIE. The IESNA 
absorbed this program for the 1983 revision of RP-8 when the pavement luminance was implemented in North America.

For the evaluation of the impact of the pavement reflection on the target visibility in this research, the program was rewritten in $\mathrm{C}+$ for this investigation. It was expanded to include the target luminance calculation from both indirect and direct sources and the target visibility level calculations. Other calculations such as visual performance, visual acuity, and discomfort glare were also added to the mathematics of the calculation for future reference but are not used in this research.

The algorithm used for the calculation is shown in Figure 90. 


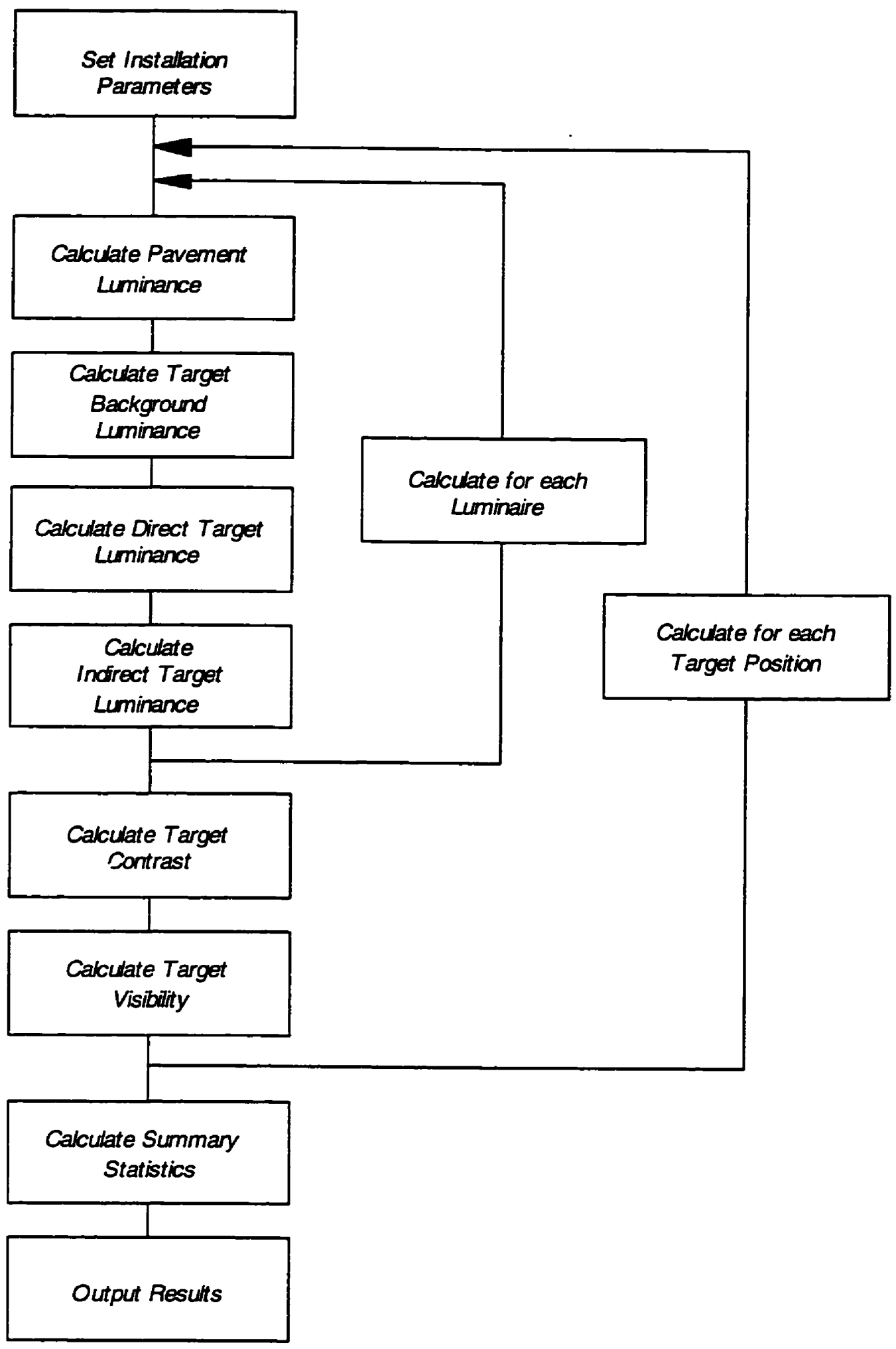

Figure 90 - Algorithm of the Pavement Luminance Calculation Program 
The algorithm begins by requesting the lighting installation parameters. The required design parameters are a calculation grid and a luminaire location grid. The calculation grid is chosen by the lighting designer to cover both the area of the roadway and the area just outside of the roadway. The IESNA RP-8 [1997] specifies criteria which the chosen calculation grid must meet. The grid of luminaire locations is recorded in the program. For each luminaire, the location of the luminaire with respect to the roadway, and the mounting characteristics of height, tilt, rotation and north/south orientation are recorded. For each luminaire, an intensity distribution table, which was previously described, is assigned based on the manufacturer and model of the luminaire. Finally, the observation point for the calculation is chosen, which is the point that represents the driver's eye location.

After the design information is placed in the system, the calculation begins. The pavement luminance and the illuminance resulting from each luminaire for each point is calculated.

Next, a target is mathematically placed at each point on the grid and the background luminance of each target is calculated. The background luminance for a target is defined as the average of the pavement luminance at the pavement point viewed just over the top of the target and at the bottom of the target. The shadowing caused by the target is not considered in the background luminance calculation. 
The calculation of the direct target illuminance according to the Equation 12 is then performed. The indirect target luminance is also calculated. A discussion of the method used for this calculation follows.

For each of the calculated results, the parameters are calculated for an individual luminaire. The result for each target is the sum of the contribution from each luminaire.

Using the Adrian model, the target contrast and the contrast threshold for each target is calculated. Finally, the visibility level is calculated for each target. The summary calculations of uniformity, average luminance and weighted visibility level are also then calculated.

The program was developed to investigate the influence of the pavement reflection on the target. Although it does calculate the required roadway lighting installation criteria, it does not consider curved or inclined roadways. Algorithms for these calculations exist but are beyond the scope of interest in this research.

The operation of the program with respect to the mathematics of the equations was verified by hand calculations for a sample installation.

There are several things which differ significantly in this program from the calculation method prescribed by the IESNA. The program developed here is a fixed observer system. This means that the observation point is a fixed position and the angular target 
size used in the visibility calculation is calculated based on the distance from the observer to the target and the height of the target. Similarly, the background luminance for each target is calculated based on a fixed observation point which means that the background luminance might actually be off of the roadway. For situations where the background is off of the roadway, the system considers the reflecting surface to still be the pavement. The IESNA method uses a moving observer. The angular target size is fixed at $10^{\prime}$ which is a $20 \mathrm{~cm}$ target at 83 metres distance. Similarly, the background luminance is calculated for points immediately behind the observer straight down the roadway. The IESNA method has been described as the "Cyclopean eye" method where the observer is infinitely wide and infinitely long. There is debate on which method is better. The method used in this research is more closely related to the European method and gives a fixed impression of the visibility in the roadway. The IESNA method assumes a fixed geometry of the observer to the roadway and tries to use a moving observer to represent the moving automobile.

The other significant difference in this program is that all of the pavement reflection values are based on the actual observer position and geometry to the roadway. Points between the angles available in the reflection are interpolated. The IESNA system uses a fixed observation angle of $1^{\circ}$ and does not interpolate the pavement reflection.

\subsection{Target Luminance Calculation}

As discussed, the target luminance is composed of direct and indirect calculations. The 
total target luminance is the sum of contributions from each luminaire. Similarly, the indirect component of the target luminance, which is the main addition made to this version of the calculation program, is based on a sum of contributions from each luminaire reflected from small areas of pavement areas in front of the target. These small areas are then summed to comprise a full integration area for the reflected light.

In order to establish the nature of the indirect portion of the target luminance, a calculation was performed using a sample lighting installation. The lighting installation was based on the Seventh Street sample roadway in Philadelphia, PA. The luminaire used in this calculation was a General Electric standard cobrahead luminaire. For this sample calculation, this luminaire was designed to be equipped with a 400 Watt Metal Halide lamp rated by Philips at 22000 lumens. The intensity distribution curve for this luminaire is file GE7320.ies. The luminaire is classified by the IESNA as a Type II luminaire. This means that the luminaire is designed to project light from the side of the road for long distances along the roadway in a fairly narrow beam spread. Five targets were calculated for the installation. The installation and target layout are shown in Figure 91. 


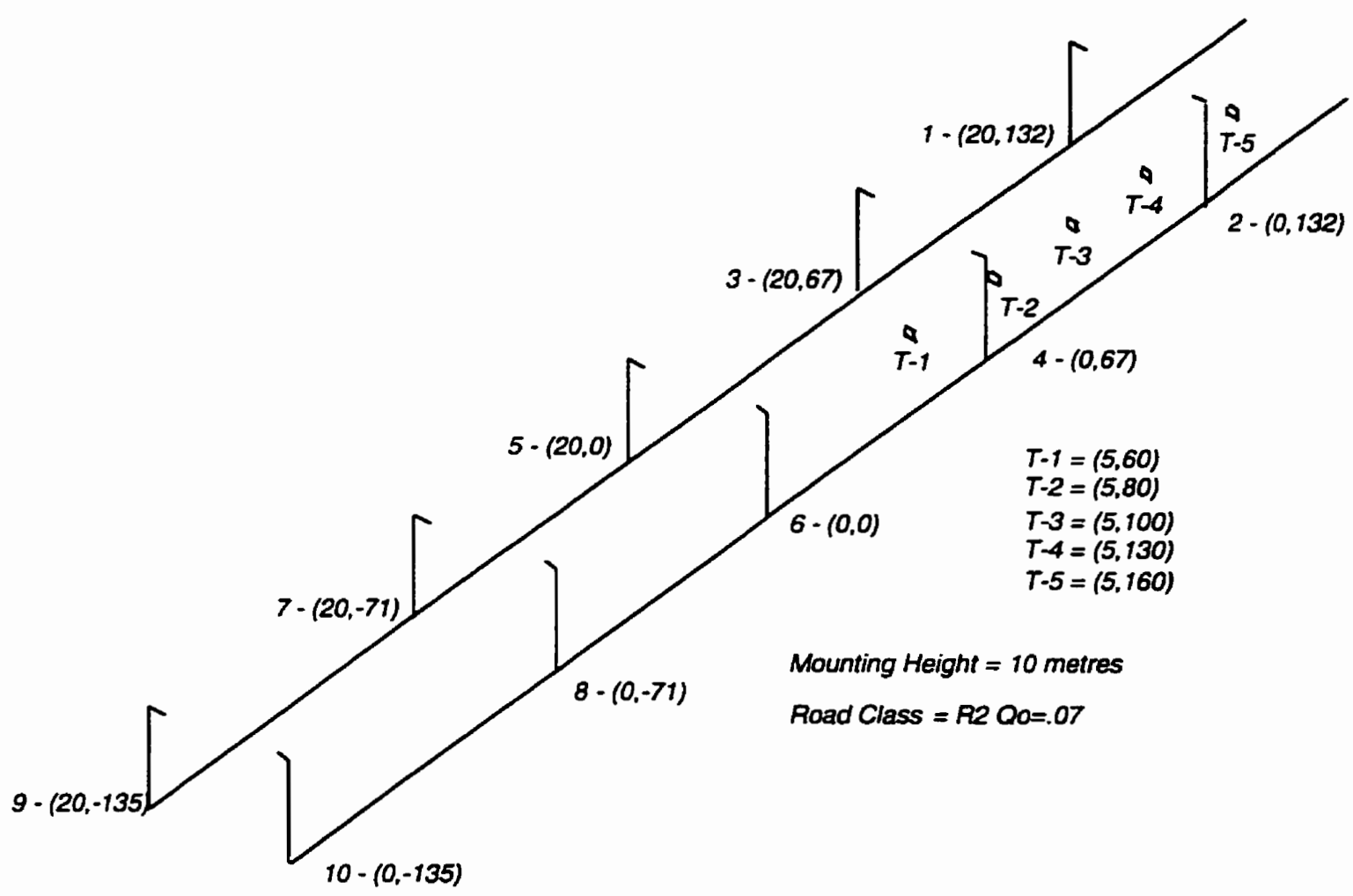

Figure 91 - Sample Installation Used for Establishing the Contribution of Reflected Light to Target Illuminance

The observer in this calculation is located at $(5,0)$ and is 1.5 metres tall.

For this installation, the results of the target luminance and the indirect and direct components are shown in Table 28. 
Table 28 - Target Luminance Results for Sample Calculation

\begin{tabular}{||c|c|c|c|}
\hline Target & Indirect & Direct & Total \\
\hline 1 & 1.96 & 0.35 & 2.31 \\
\hline 2 & 3.16 & 19.76 & 22.92 \\
\hline 3 & 4.49 & 6.04 & 10.53 \\
\hline 4 & 2.18 & 0.31 & 2.49 \\
\hline 5 & 5.76 & 11.66 & 17.42 \\
\hline
\end{tabular}

The development of the calculation method for the indirect portion follows.

\subsubsection{Pavement Area Contributions}

In the calculation of the target luminance, the contribution of the pavement reflection is based on the sum of the reflection from a number of pavement areas in front of the target. These pavement areas are chosen based on the target size. An example of this grid is shown in Figure 92. The size of the integration area and the size of each reflecting surface can be scaled by some factor, $\mathrm{X}$, of the target size. 


\section{Target}

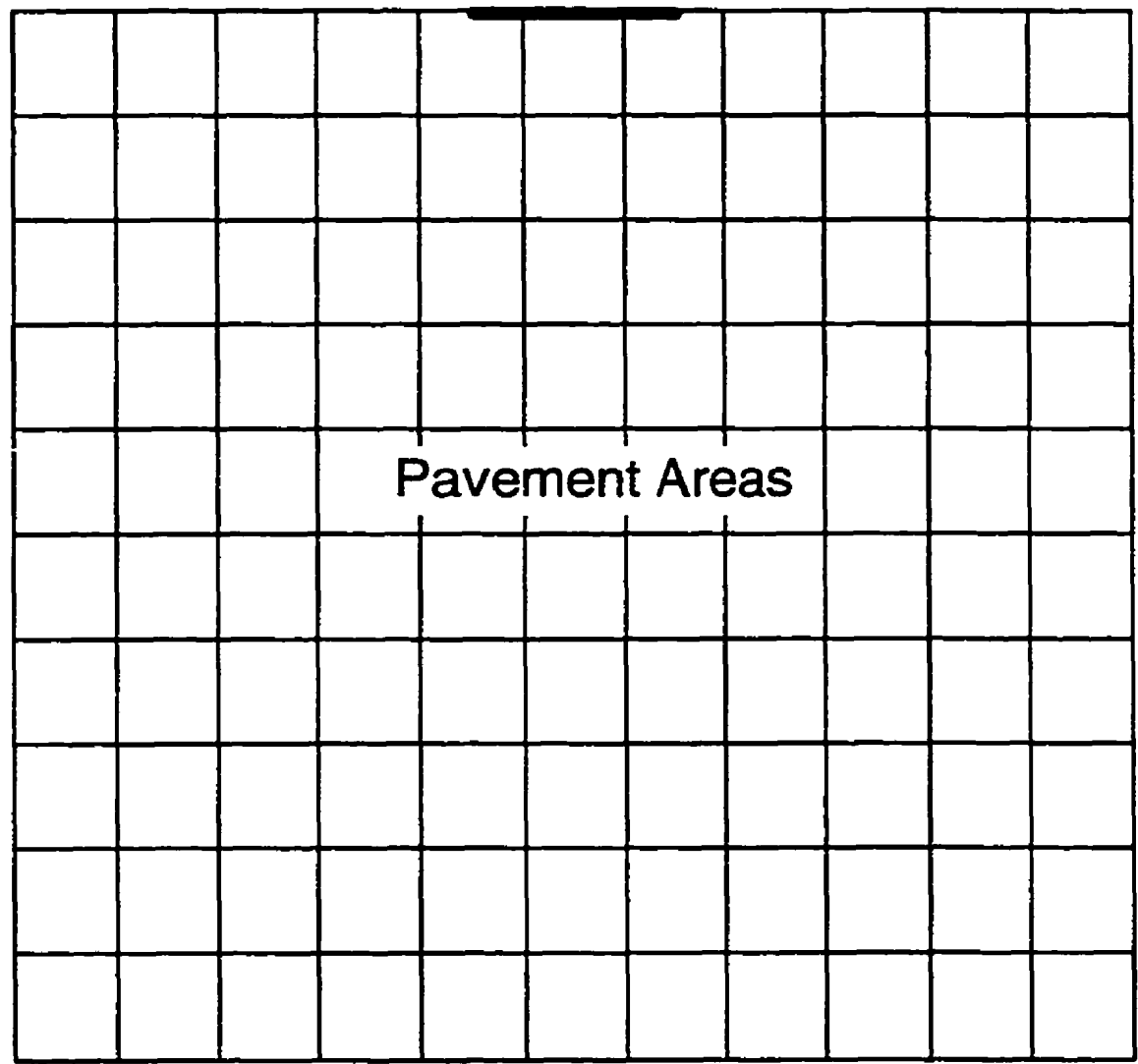

Grid Size $=X \bullet$ Target Width

Figure 92 - Sample Indirect Calculation Grid

The size and nature of this grid was one of the aspects of the calculation algorithm which was investigated using this calculation program. Obviously, the larger the calculation area and the smaller the pavement area, the greater the accuracy of the integration of the reflection from the surface. Two things must be considered when establishing the limits of the reflection integration. They are the shape and the size of the integration area.

The basic shape of the integration area was established as a square in front of the target. Based on this, calculations were performed using the sample lighting installation to 
establish the contribution of every reflecting area in the integration field to the illuminance of each target. These contributions are shown in Figure 93.

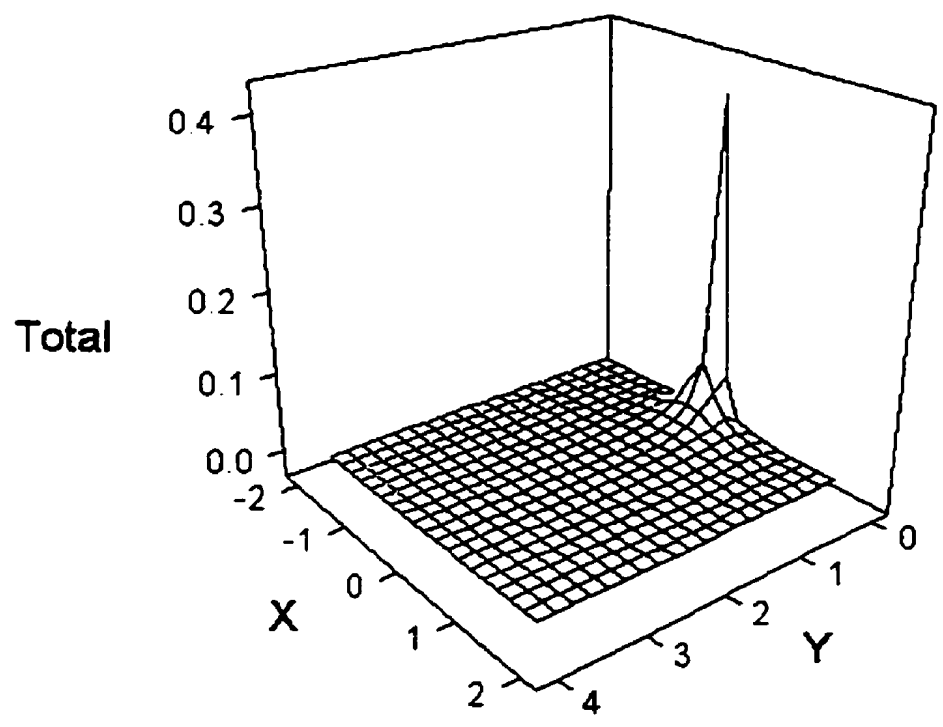

Figure 93 - Total Indirect Contribution to Target Iluminance in lux

In Figure 93, the target would be located at the origin, $(0,0)$, of the field. The dimensions $\mathrm{X}$ and $\mathrm{Y}$ represent the distance from and to the sides of the target. The vertical axis in the graph is the contributed illuminance on the target in lux. From the diagram, it is evident that the total contributing area is relatively small. The goal of the choice of integration grid is to have every square contributing in some way to the total reflection. In order to fully establish this contribution, the effect from each of the luminaires in the calculation was also considered. Figures 94 and 95 show the contribution from Luminaire 4 and Luminaire 10 to Target 1. 


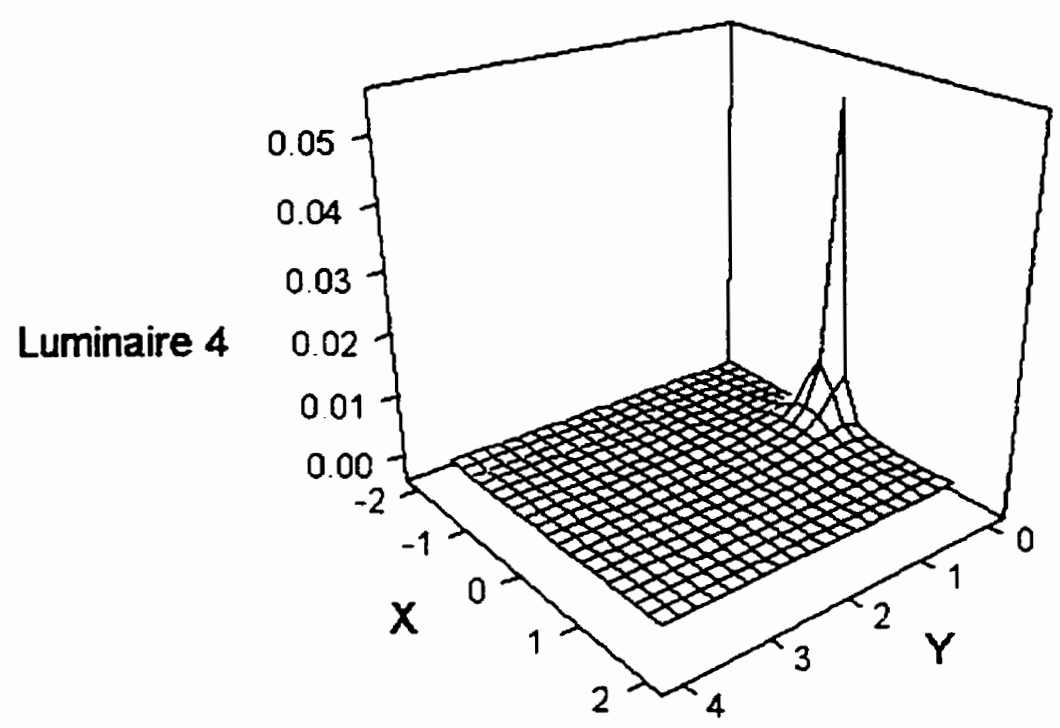

Figure 94 - Indirect Contribution of Luminaire 4 to Target 1 Iluminance 


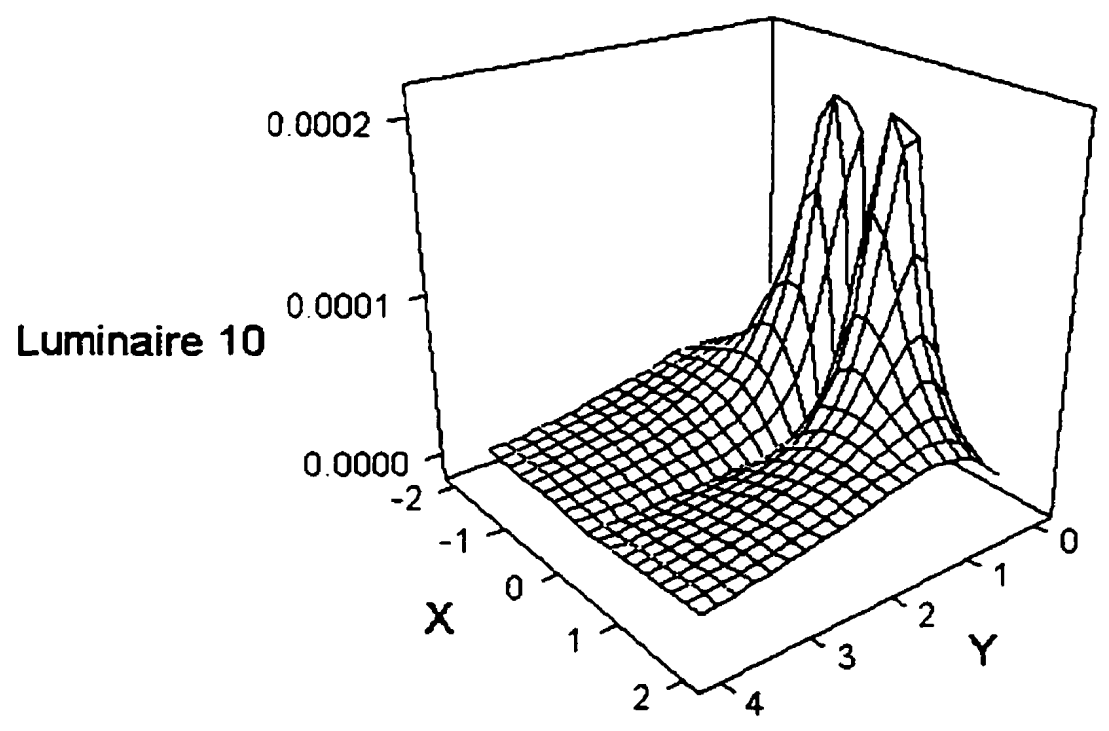

Figure 95 - Indirect Contribution of Luminaire 10 to Target 1 Illuminance

The contribution from Luminaire 10 shows that the entire field takes part in the reflected illuminance. Based on these contribution results, it was decided that a square integration area would be used for all the calculations. The grid chosen was 20 squares deep by 20 squares wide.

\subsubsection{Pavement Area Size}

To establish the required size of reflecting area, calculations were performed with a calculation grid which was scaled by several different multiples of the target size. The same sample installation was used for this evaluation. The multiples were made on each dimension of the square, which means that doubling the multiple would double the linear 
dimension of each side of the square and quadruple the reflecting area. The direct component of the target illuminance remained unchanged in this calculation, but the indirect component of the target illuminance increased with an increase in the size of the reflecting components. The results are shown in Figure 96.

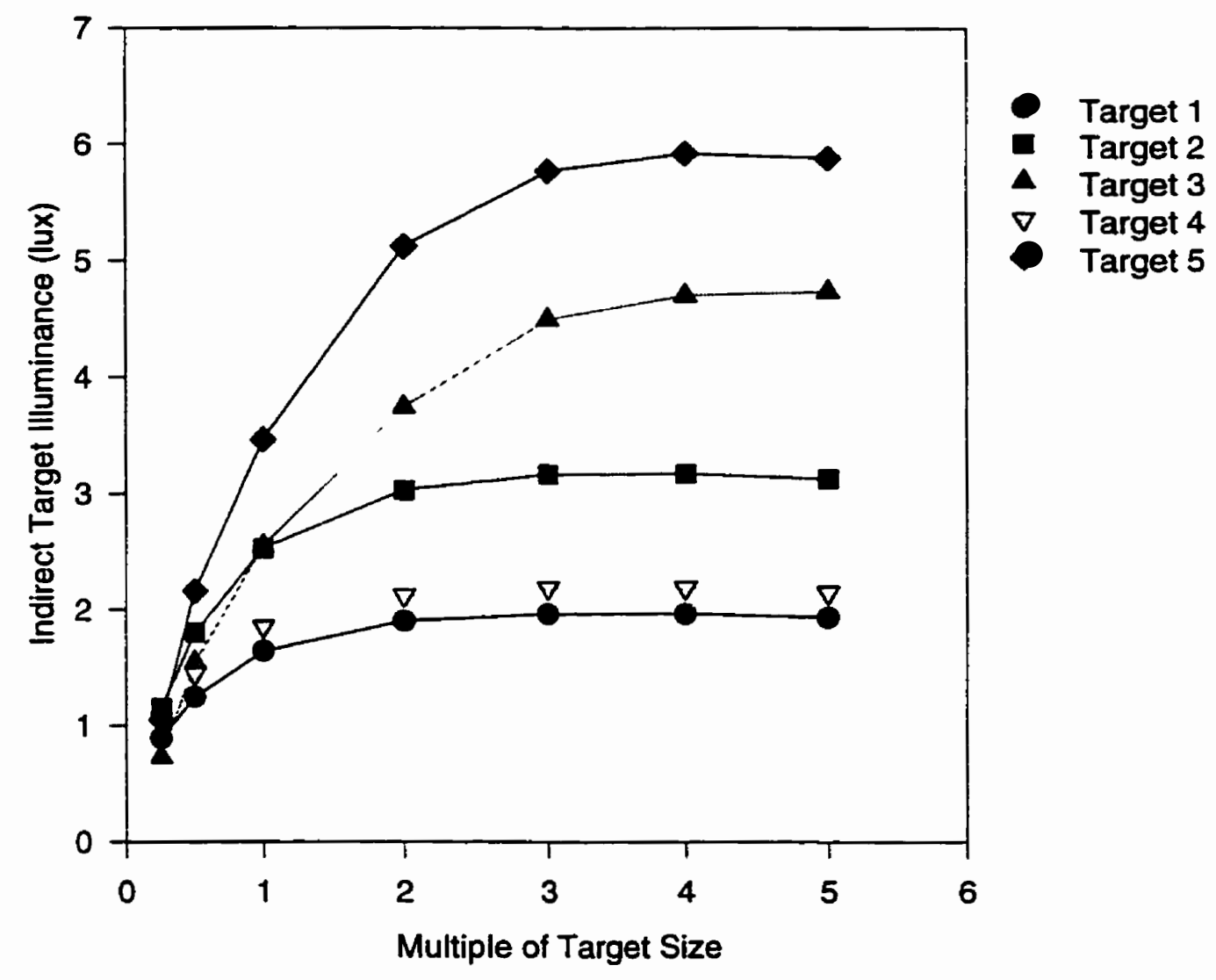

Figure 96 - Indirect Target Iluminance versus Size of Reflection Integration Area The target illuminance reaches a maximum. Using this maximum, the percentage of the target illuminance achieved by each grid size multiple was determined. These results are 
shown in Figure 97.

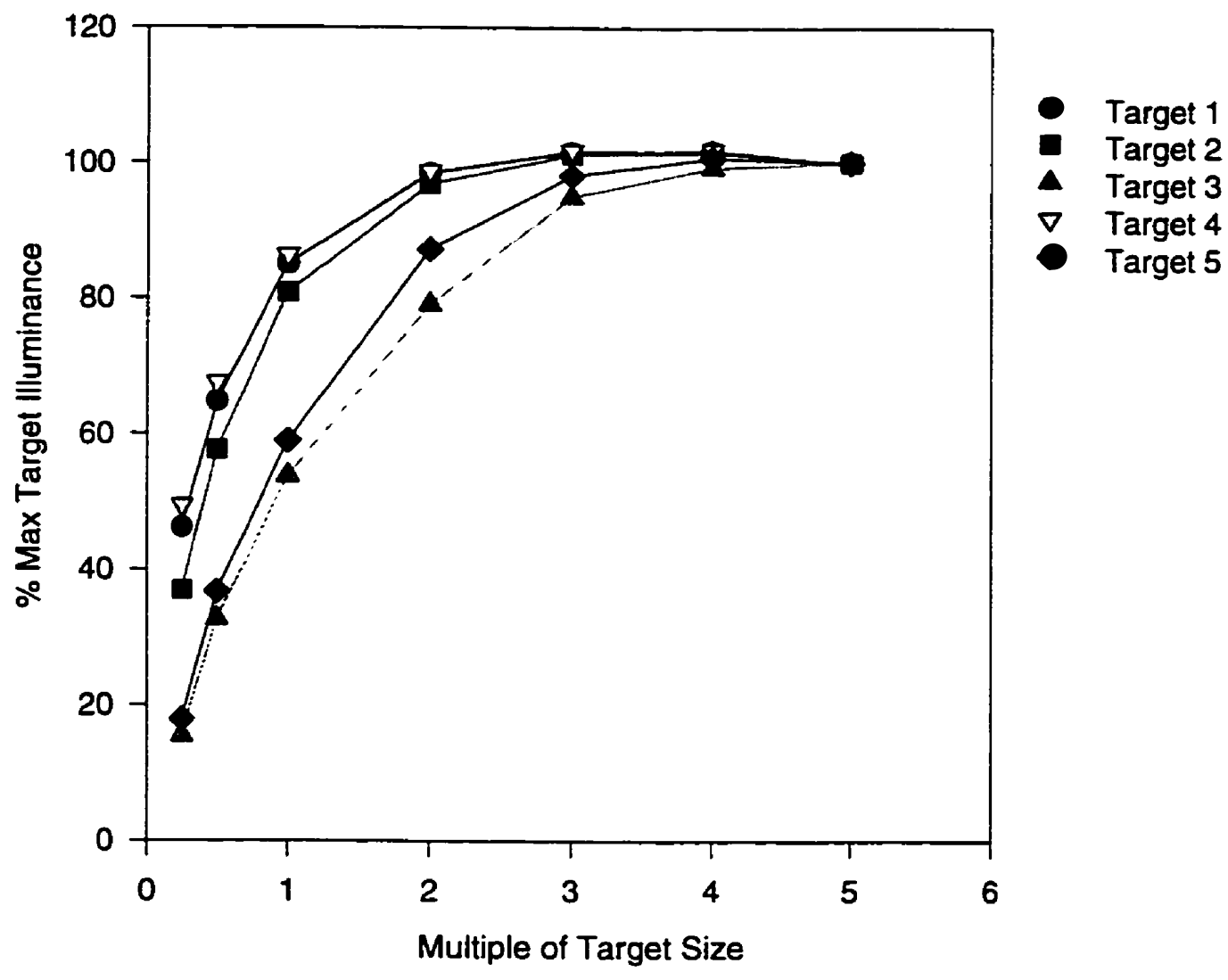

Figure 97 - Percent of Maximum Target Iluminance versus Size of Reflection Integration Area

To achieve at least $90 \%$ of the maximum target illuminance, a grid multiple of three was chosen as the required value. Using this value of multiplier, the integration area was established. A further verification of this value was performed by calculating the indirect target illuminance with the same overall size of integration area composed of many more, smaller reflecting areas. These results for a calculation with the grid size 
multiple of one, are shown in Table 29.

Table 29 - Comparison of Indirect Contribution of Large to Small Reflection Areas

\begin{tabular}{||c|r|r|}
\hline \hline Target & \multicolumn{1}{|c|}{ Large } & \multicolumn{1}{c|}{ Small } \\
\hline 1 & 1.96 & 1.96 \\
\hline 2 & 3.16 & 3.19 \\
\hline 3 & 4.49 & 4.71 \\
\hline 4 & 2.18 & 2.27 \\
\hline 5 & 5.76 & 5.88 \\
\hline
\end{tabular}

These results show that the indirect target illuminance is generally the same for the two target calculation methods. The difference lies in the number of operations which are required. For the grid multiple of three, 400 integration areas were calculated, while with the grid multiple of one, 1806 pavement areas were considered. Obviously, the fewer number of calculations is most desirable in this evaluation.

For all calculations made, the reflection integration area was 20 by 20 squares with a grid multiple of three or an area of nine times the target size.

\subsubsection{Luminaire Contributions}

The contribution of each luminaire to the indirect target illuminance was used to define the number of luminaires to include in the calculation. Using the sample installation, the contribution of each luminaire was calculated based on the distance from each luminaire to the target. The calculation was also performed for different luminaire spacing to mounting height ratios. These results are shown in Figure 98 and include all five targets 
in the sample installation.

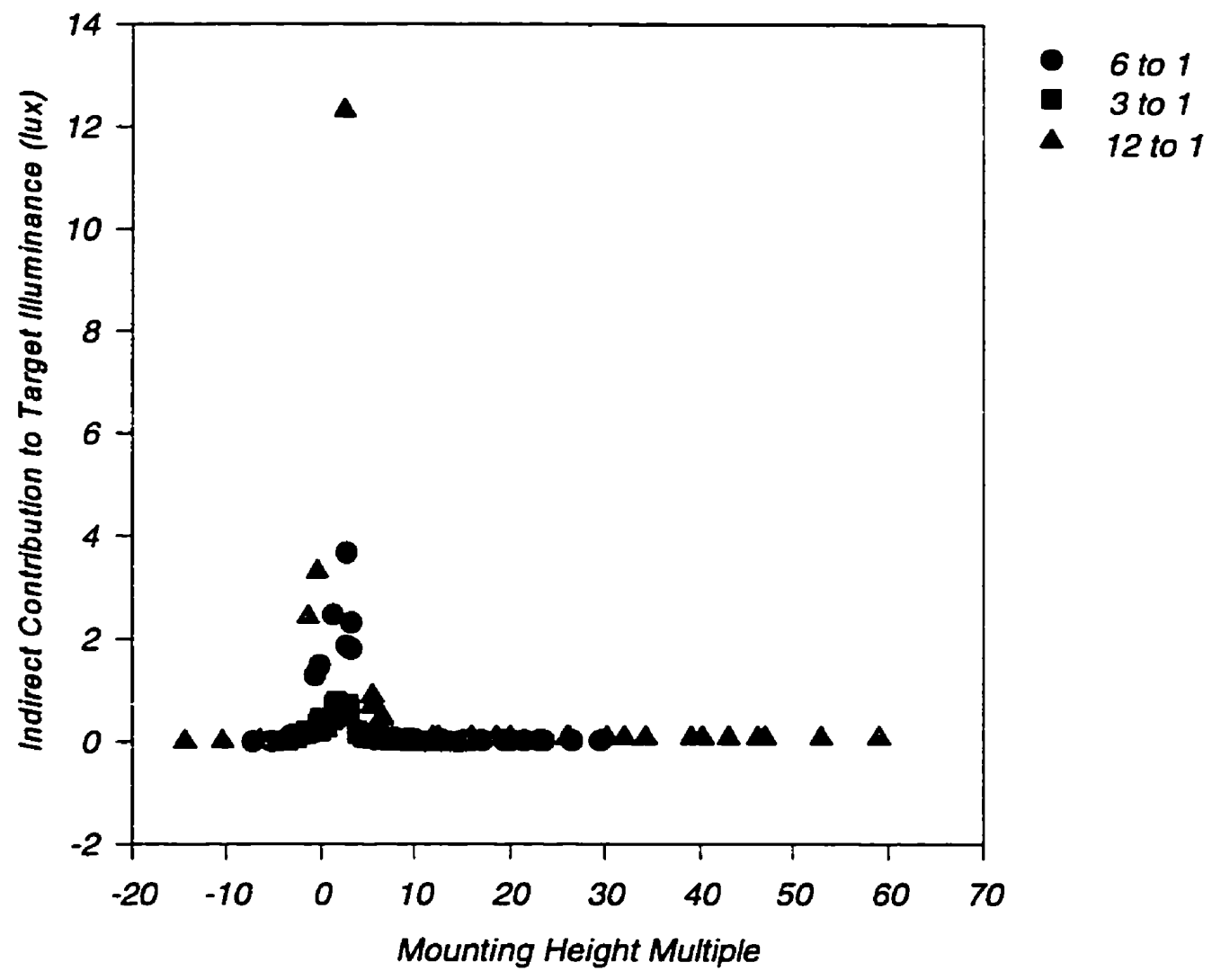

Figure 98 - Contribution of Each Luminaire to Indirect Target Iluminance versus Multiple of Mounting Height Distance from Target for Three Spacing to Mounting Height Ratios

These results show that only the luminaires closest to the target have a significant contribution to the target reflection. The results also show that the influence of luminaires beyond ten mounting heights distance from the target are not contributing to 
the indirect target illuminance. This means that only a few of the luminaires in the design need to be calculated for the determination of the indirect target illuminance. The other significant aspect of this calculation is the contribution of the luminaires which are beyond the target. These are shown in Figure 98 with negative distance to the target.

\subsection{Comparison to Measured Results}

As mentioned, experiments have been carried out in which the illuminance and the luminance of the pavement and targets have been measured and compared to the calculated results, particularly by the IESNA VTF. These experiments were generally undertaken on test roadways in several different countries. These test sites were and still are used by luminaire manufacturers and lighting designers to both test new designs and determine the relationships between the design quality criteria, whether it is luminance or visibility level, and actual driver performance. Since the inception of the visibility level as the quality criterion, these road sections have been used to both measure the target luminance and to estimate the contribution of the pavement reflection on the calculated results.

Three basic experiments in this field have been performed: the first in Philadelphia, PA, the second in Hendersonville, NC, and the third in Scottsdale, AZ. In order to fully evaluate the functionality of the calculation program and the pavement reflection results developed in this research, the results of the calculations and the measurements will be compared. 


\subsubsection{Philadelphia, Pennsylvania}

This first experiment was performed by the VTF of the IESNA Roadway Lighting Committee. This experiment and the Hendersonville, NC experiment, are documented in Keck[1992]. This experiment was an extension of the work performed by Janoff [1991]. As mentioned, the roadway used in Philadelphia is a section of Seventh Street which has been designed with individual control of the luminaires. This section of roadway has been used for several years in experimentation with drivers and driver visibility. Janoff's work was an experiment to relate visibility of targets and visual performance. $\mathrm{He}$ highlighted several problems by obtaining consistent photometry results of the pavement and the target luminance. Based on the Janoff results, the VTF began the task of identifying the cause of the photometry changes and comparing these results to calculated results.

The pavement section for this experiment is 300 metres long. The roadway is 20 metres wide and has a double-sided opposite luminaire installation. There are ten luminaires in the roadway stretch with five on each side of the roadway spaced seventy metres apart. The mounting height is ten metres with a 2.5 metre overhang. Targets for the measurement experiment were placed just before and just after the fourth luminaire. This layout is shown in Figure 99. 


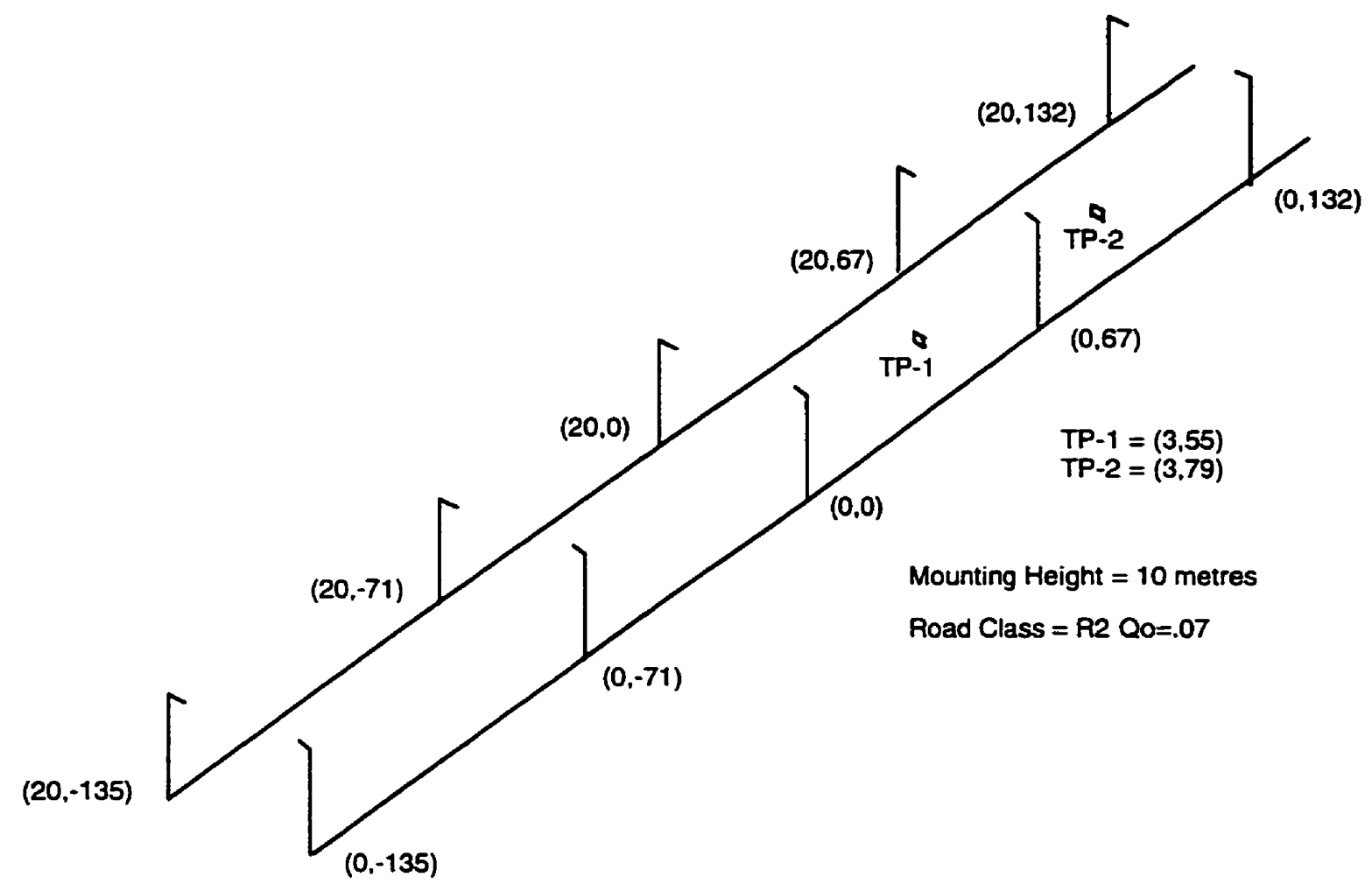

Figure 99 - Design Layout for the Philadelphia Seventh Street Installation

There are several difficulties with the documentation of this experiment. The most significant is that the mounting height, lamp type and the luminaire type were not measured or described. It is known that the luminaires were General Electric but the model was not specified. For these calculations, the GE7320.ies distribution was used at a mounting height of ten metres.

In the calculations for this experiment, the luminaire intensity was scaled by a factor determined from a comparison of the average illuminances on the pavement. The average illuminance from the measured and the calculated results were set to be equal by adjusting the intensity of the lamp used in the calculation. The comparison of the calculated versus measured results are shown in Table 30. 
Table 30 - Comparison of Measured Iluminance to Scaled Calculated Illuminance for Philadelphia Experiment

\begin{tabular}{|c|c|c|c|c|}
\hline $\mathrm{Y}$ & $\bar{X}$ & Measured & Calculated & \%Difference \\
\hline 387 & 9 & 7.4 & 11.36 & -53.51 \\
\hline 387 & 63 & 8.0 & 15.09 & -88.62 \\
\hline 340 & 33 & 5.4 & 7.27 & -34.63 \\
\hline 340 & 39 & 5.1 & 7.33 & -43.72 \\
\hline 324 & 33 & 6.0 & 6.11 & -1.83 \\
\hline 324 & 39 & 5.8 & 6.16 & -6.20 \\
\hline 277 & 9 & 5.6 & 9.64 & -72.14 \\
\hline 277 & 63 & 8.1 & 12.84 & -58.52 \\
\hline 165 & 9 & 8.2 & 9.84 & -20.00 \\
\hline 165 & 63 & 5.6 & 13.05 & -133.0 \\
\hline 119 & 33 & 6.0 & 5.53 & 7.83 \\
\hline 119 & 39 & 5.7 & 5.62 & 1.40 \\
\hline 103 & 33 & 6.3 & 5.33 & 15.39 \\
\hline 103 & 39 & 5.9 & 5.43 & 7.96 \\
\hline 56 & 9 & 7.7 & 9.72 & -26.23 \\
\hline 56 & 63 & 6.3 & 12.91 & -104.92 \\
\hline-55 & 9 & 10.2 & 13.3 & -30.39 \\
\hline-55 & 63 & 9.3 & 13.11 & -40.96 \\
\hline-102 & 33 & 9.8 & 4.70 & 52.04 \\
\hline-102 & 39 & 9.8 & 4.75 & 51.53 \\
\hline-118 & 33 & 10.2 & 3.26 & 68.03 \\
\hline-118 & 39 & 9.9 & 3.32 & 66.46 \\
\hline-165 & 9 & 11.2 & 7.35 & 34.38 \\
\hline-165 & 63 & 8.2 & 10.00 & -21.95 \\
\hline-289 & 9 & 9.9 & 9.86 & 0.40 \\
\hline-289 & 63 & 16.8 & 13.06 & 22.26 \\
\hline-336 & 33 & 10.2 & 6.69 & 34.41 \\
\hline-336 & 39 & 11.4 & 6.81 & 40.26 \\
\hline-352 & 33 & 11.1 & 7.92 & 28.64 \\
\hline-352 & 39 & 11.5 & 8.05 & 30.00 \\
\hline-399 & 9 & 17.4 & 12.62 & 27.47 \\
\hline-399 & 63 & 14.8 & 16.86 & -13.92 \\
\hline \multirow{2}{*}{\multicolumn{2}{|c|}{ Average }} & 8.9 & \begin{tabular}{|l|}
8.9 \\
\end{tabular} & -8.19 \\
\hline & & & Std Dev & 48.46 \\
\hline
\end{tabular}


The average of the measured and calculated illuminance were set to be equal, accounting for the intensity of the lamp. The average deviation of measured to calculated across the calculation grid is $-8.19 \%$ with a standard deviation of $48.5 \%$. The VTF does not specify an average deviation but found a standard deviation of $38 \%$. The higher error in these results is due to the different intensity distribution file used for the calculation. It would be expected that if the correct intensity table, lamp and mounting height were used, the illuminance would have a lower deviation.

The next step of the experiment was to measure the pavement luminance at three distances: $22.5,82.5$ and 232.5 metres. The measurements were made in reference to a target with the photometer aimed just over the top of the target and with the photometer aimed just below the base of the target. The calculated and measured results from the program are shown in Table 31. 
Table 31 - Comparison of Measured Pavement Luminance to Calculated Pavement Luminance for Philadelphia Experiment

\begin{tabular}{||c|c|c|r|r|r|}
\hline Dist & Target & Position & Measured & Calculated & \multicolumn{1}{c|}{ \%Diff } \\
\hline 22.5 & 1 & In Front & 0.89 & 7.08 & 696.6 \\
\hline 82.5 & 1 & In Front & 1.23 & 5.43 & 341.8 \\
\hline 232.5 & 1 & In Front & 2.91 & 4.68 & 60.87 \\
\hline 22.5 & 1 & Over Top & 0.75 & 7.40 & 887.3 \\
\hline 82.5 & 1 & Over Top & 2.91 & 4.05 & 39.17 \\
\hline 232.5 & 1 & Over Top & 1.67 & 1.69 & 1.520 \\
\hline 22.5 & 2 & In Front & 1.99 & 3.62 & 82.00 \\
\hline 82.5 & 2 & In Front & 2.75 & 2.50 & 9.070 \\
\hline 232.5 & 2 & In Front & 3.15 & 2.12 & 32.72 \\
\hline 22.5 & 2 & Over Top & 2.47 & 3.09 & 24.95 \\
\hline 82.5 & 2 & Over Top & 1.27 & 1.86 & 46.73 \\
\hline 232.5 & 2 & Over Top & 2.5 & 5.08 & 103.4 \\
\hline
\end{tabular}

The table shows that the measured luminance results vary with distance. The luminance is measured in candela per metre squared and does not vary with distance as the candela is measured in terms of solid angle which is constant over distance. Two things contribute to the variation in the measurement. The first is the projection of the solid angle on the pavement surface which does vary with distance. The second is the aim of the photometer. A photometer has two aspects which are critical for use; the first is the acceptance angle and the second is the aim point. The acceptance angle of a photometer determines the solid angle over which the luminance measurement is averaged. The aim point is the centre of the acceptance angle, which is used to point the photometer at the object of interest. Since the measurements of pavement luminance are made at a $1^{\circ}$ down observation angle, this means that the solid angle of acceptance of the luminaire is actually an ellipse on the pavement surface. At the 232.5 metre measuring distance, the 
ellipse projected on the pavement surface is $\mathbf{2 5}$ metres in length where the ellipse at the 22.5 metre distance is .3 metres in length. This means that the photometer is averaging over a much larger pavement area for one distance than the other. Similarly, when the photometer is aimed over the top of the target at 232.5 metres, the centre point of the ellipse is in a much different position than at the shorter distances. This means that the measured results have some difficulties in them. The use of the photometer, the environment and the size of the acceptance angle for any measurement experiment must be carefully controlled in order for these measurements to be performed effectively.

There seems to be very little relationship of the calculated results to the measured results. The percentage difference between the measured and the calculated results are also shown in Table 32. These differences are great and are likely due to the difference in the luminaire distribution and the actual installed luminaire.

The final portion of this experiment was the evaluation of target luminance. For this experiment, the target luminance was measured at the two target positions with five different reflectances. The calculated results compared to the measured results are shown in Table 32. 
Table 32 - Comparison of Measured Target Luminance to Calculated Target Luminance

\begin{tabular}{||r|r|r|r|r|r|r|}
\hline Dist & Target & Reflectivity & Measured & Calculated & \% Diff. & \% Reduc. \\
\hline 82.5 & 1 & 0.05 & 0.38 & 0.084 & 77.8 & 88.8 \\
\hline 82.5 & 1 & 0.20 & 0.97 & 0.336 & 65.3 & 71.5 \\
\hline 82.5 & 1 & 0.30 & 1.37 & 0.505 & 63.1 & 59.7 \\
\hline 82.5 & 1 & 0.50 & 2.3 & 0.841 & 63.4 & 32.35 \\
\hline 82.5 & 1 & 0.80 & 3.4 & 1.346 & 60.4 & 0.00 \\
\hline 82.5 & 2 & 0.05 & 0.15 & 0.889 & -492.9 & 62.5 \\
\hline 82.5 & 2 & 0.20 & 0.21 & 3.557 & -1594 & 47.5 \\
\hline 82.5 & 2 & 0.30 & 0.27 & 5.336 & -1876 & 32.5 \\
\hline 82.5 & 2 & 0.50 & 0.4 & 8.894 & -2123 & 0.00 \\
\hline 82.5 & 2 & 0.80 & 0.4 & 14.23 & -3457 & 0.00 \\
\hline
\end{tabular}

In this table, the results again show little relationship between the measured data and the calculated data. The table provides the percentage difference between the measured and the calculated results. For target number 1 , the percent difference is relatively stable for all of the reflectances of the target. This implies that although the absolute value of the calculated results is very different than the measured, the relative relationship of the data points is the same. For Target 2, the percent difference is not the same. This is the result of anomalies in the measurement of the target. The percentage reduction of the target luminance from the $80 \%$ reflectance levels to the $5 \%$ is also given in the table. The luminance of the target should be proportional to the reflectance of the target. This is not the case for Target 2. This implies either an error in the measurement of the target itself or in the measurement of the target reflectivity. 


\subsubsection{Hendersonville, North Carolina}

This experiment, documented in Keck[1992], was an extension of the VTF Philadelphia experiment and established the impact of reflected light on the target luminance by evaluating both the reflected portion of the illuminance and the direct portion. To separate the reflected and direct portion of the target luminance, a piece of velvet was used to limit reflected pavement illuminance and a shadow caster was used to limit direct illuminance.

In this experiment, the luminaires were carefully photometered and installed, and the target reflection was carefully characterized. The test set up for this experiment is shown in Figure 100. Like the Philadelphia experiment, the details of the installation were poorly documented. The luminaire height, the lamp, target reflectance and the pavement type were chosen as a "best guess". Again, the GE7320.ies luminaire intensity distribution file was used. 


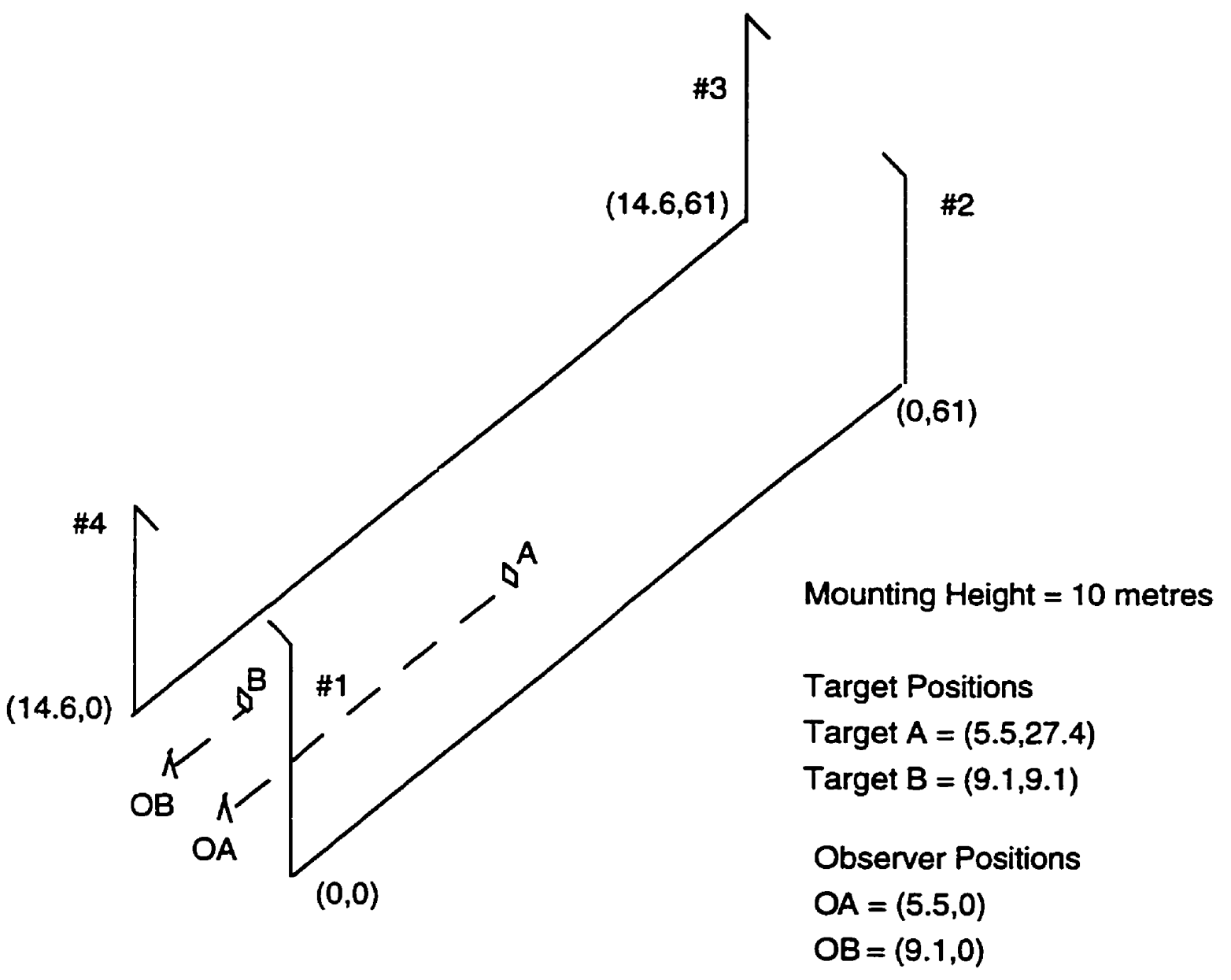

Figure 100 - Lighting Layout of Hendersonville Experiment

To study the influence of reflected light on the target luminance, several measurements were made each with only a single luminaire lit at a time. The target luminance was then measured. Then a shadow caster which blocked direct light from the luminaire onto the target was set up and the target luminance measured again. This establishd the direct portion of the target luminance. Next, a piece of velvet was placed on the pavement to block reflected light with the shadow caster in place. The velvet was moved to various locations in front of the target and the target luminance was measured at each location, thus evaluating the influence of the various pavement sections on the target luminance. 
The section positions are shown in Figure 101. This process was performed for two different target points each with a different observation point. Calculations were performed for each of these configurations. The results of the measured and the calculated results are shown in Table 33.

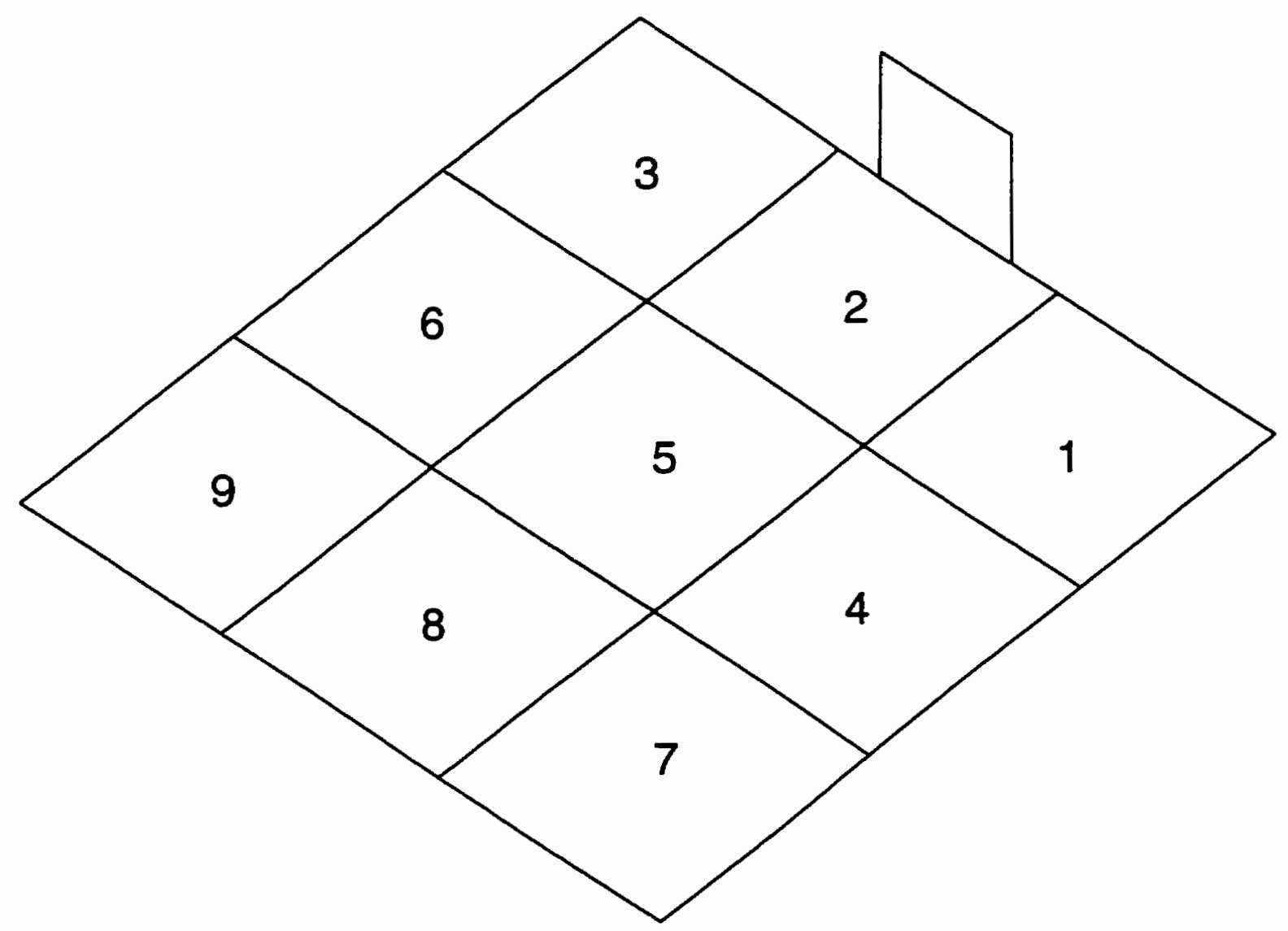

Figure 101 - Reflection Grid Layout for Hendersonville Experiment 
Table 33 - Comparison of Calculated Results to Measured for Hendersonville Experiment

\begin{tabular}{|c|c|c|c|c|c|c|c|}
\hline Luminaire & Shadow & Velvet & Target & Measured & Calculated & $\begin{array}{c}\% \text { of } \\
\text { Total- } \\
\text { meas }\end{array}$ & $\begin{array}{c}\% \text { of } \\
\text { Total - } \\
\text { calc }\end{array}$ \\
\hline 1 & $\mathrm{n}$ & $\mathrm{n}$ & 1 & 6.85 & 1.646 & & \\
\hline 1 & $y$ & $\mathrm{n}$ & 1 & 0.28 & 0.162 & & \\
\hline 1 & $y$ & 2 & 1 & 0.13 & 0.093 & 46.4 & 57.2 \\
\hline 1 & $y$ & 5 & 1 & 0.28 & 0.151 & 100 & 92.9 \\
\hline 1 & $y$ & 4 & 1 & 0.28 & 0.153 & 100 & 94.3 \\
\hline 1 & $y$ & 8 & 1 & 0.28 & 0.158 & 100 & 97.3 \\
\hline 1 & $y$ & 7 & 1 & 0.28 & 0.158 & 100 & 97.4 \\
\hline 2 & $\mathrm{n}$ & $\mathbf{n}$ & 1 & 0.23 & 0.0114 & & \\
\hline 2 & $\mathrm{n}$ & 2 & 1 & 0.19 & 0.0029 & 82.6 & 25.9 \\
\hline 2 & $\mathrm{n}$ & 5 & 1 & 0.22 & 0.0108 & 95.7 & 95.1 \\
\hline 2 & $\mathrm{n}$ & 1 & 1 & 0.21 & 0.0108 & 91.3 & 95.1 \\
\hline 2 & $\mathrm{n}$ & 4 & 1 & 0.23 & 0.0111 & 100 & 96.9 \\
\hline 2 & $\mathrm{n}$ & 8 & 1 & 0.21 & 0.0113 & 91.3 & 98.9 \\
\hline 2 & $\mathrm{n}$ & 6 & 1 & 0.22 & 0.0112 & 95.7 & 97.9 \\
\hline 3 & $\mathrm{n}$ & $\mathrm{n}$ & 1 & 0.25 & 0.0152 & & \\
\hline 3 & $\mathrm{n}$ & 2 & 1 & 0.17 & 0.0039 & 68.0 & 26.2 \\
\hline 3 & $\mathrm{n}$ & 5 & 1 & 0.25 & 0.0145 & 100 & 95.1 \\
\hline 3 & n & 8 & 1 & 0.26 & 0.0151 & 104 & 98.9 \\
\hline 3 & n & 6 & 1 & 0.25 & 0.0148 & 100 & 97.0 \\
\hline 4 & $\mathrm{n}$ & $\mathrm{n}$ & 1 & 5.22 & 1.767 & & \\
\hline 4 & $y$ & $\mathrm{n}$ & 1 & 2.45 & 0.1599 & & \\
\hline 4 & $y$ & 2 & 1 & 1.21 & 0.0911 & 49.4 & 56.9 \\
\hline 4 & $y$ & 5 & 1 & 2.27 & 0.1502 & 92.7 & 93.9 \\
\hline 4 & $y$ & 8 & 1 & 2.31 & 0.1571 & 94.3 & 98.2 \\
\hline 4 & $y$ & 6 & 1 & 2.31 & 0.1523 & 94.3 & 95.1 \\
\hline 4 & $y$ & 9 & 1 & 2.31 & 0.1569 & 94.3 & 98.1 \\
\hline 1 & $\mathrm{n}$ & $\mathrm{n}$ & 2 & 15.01 & 1.869 & & \\
\hline 1 & $y$ & $\mathrm{n}$ & 2 & 1.45 & 0.1454 & & \\
\hline 1 & $y$ & 2 & 2 & 0.52 & 0.0584 & 35.8 & 40.2 \\
\hline 1 & $y$ & 5 & 2 & 1.45 & 0.1382 & 100 & 95.1 \\
\hline 1 & $y$ & 7 & 2 & 1.48 & 0.1438 & 102 & 99.3 \\
\hline 1 & $y$ & 8 & 2 & 1.49 & 0.1437 & 103 & 98.8 \\
\hline
\end{tabular}


The comparison results show again very little similarity in the absolute values of the target luminance. The aspect of interest here was the relative contribution of the pavement areas to the target luminance. For each of the targets and the velvet combinations, the percentage of the total indirect target luminance was calculated. When comparing the nature of the percent reduction, the calculated and the measured results both show a very similar nature. These results, although not comparing absolutely, verify the contribution of the reflected pavement to the target luminance.

\subsubsection{Scottsdale, Arizona}

The third experiment of this nature was an evaluation of measurement differences presented in Lewin [1993]. In this experiment, 36 rows of target were placed along a roadway, one row every 3 metres. Four targets were placed in each row accross the roadway with 2 metre betwwn each target. The luminaires in this installation were 120 metres apart. The first row of targets began immediately behind the first row of luminaires and the last row finished just in front of the last set of luminaires. This arrangement is shown in Figure 102. The lamps were identified as Cobrahead luminaires with a 250 Watt Metal Halide source. The exact make and model of the lamps was not given, so the GE7320.ies luminaire file was used. 


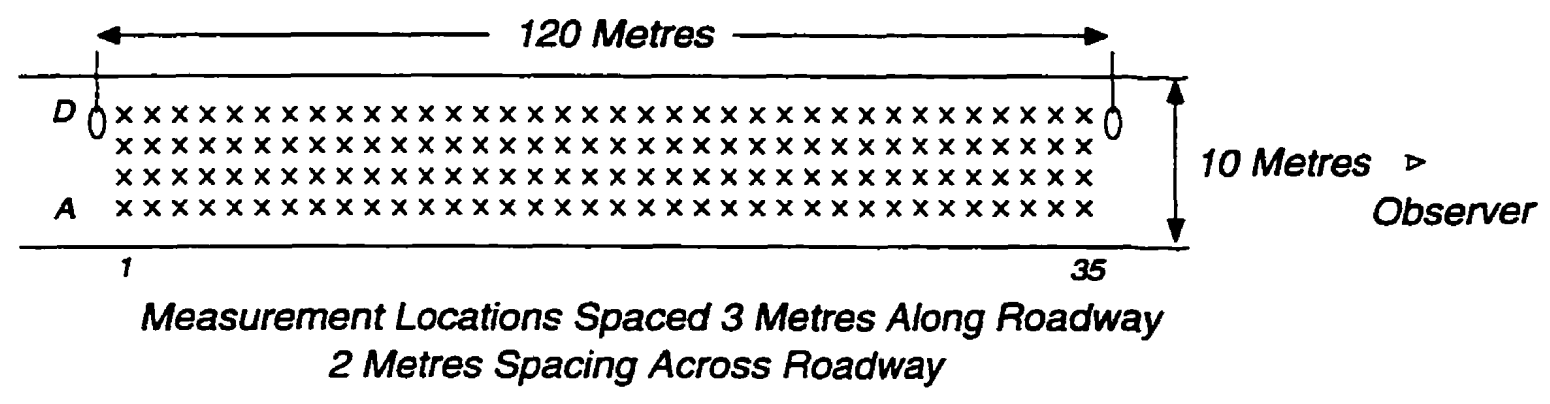

Figure 102 - Roadway Layout for Scottsdale Experiment

The direct and the total target luminance was evaluated in the same way as the

Hendersonville experiment. A piece of black velvet was placed in front of the target and then another target luminance measurement was made. Calculations using the program were also made in this configuration. The ratio of the direct to the total luminance for the measurement and the calculation are shown in Table 34. 
Table 34 - Comparison of Measured to Calculated Results for Scottsdale Experiment

\begin{tabular}{||r|r|r|}
\hline & \multicolumn{2}{|c|}{ Ratio } \\
\hline Target & Measured & Calculated \\
\hline A1 & 1.69 & 34.16 \\
\hline B1 & 2.69 & 48.68 \\
\hline C1 & 3.55 & 60.62 \\
\hline D1 & 3.01 & 55.33 \\
\hline A10 & 1.06 & 11.23 \\
\hline B10 & 1.13 & 11.38 \\
\hline C10 & 1.15 & 10.64 \\
\hline D10 & 1.12 & 9.61 \\
\hline A20 & 1.00 & 2.30 \\
\hline B20 & 1.00 & 2.39 \\
\hline C20 & 1.03 & 2.49 \\
\hline D20 & 1.15 & 2.51 \\
\hline A30 & 1.04 & 1.33 \\
\hline B30 & 1.02 & 1.37 \\
\hline C30 & 1.04 & 1.42 \\
\hline D30 & 1.02 & 1.46 \\
\hline A35 & 1.11 & 1.19 \\
\hline B35 & 1.09 & 1.18 \\
\hline C35 & 1.07 & 1.17 \\
\hline D35 & 1.07 & 1.18 \\
\hline
\end{tabular}

For the target positions just ahead of the luminaire, Target row 1 , the indirect

contribution is the greatest. This is the target position which has the least direct

contribution from the luminaire in front of it and the greatest indirect contribution from the luminaire behind it. This is evident in the calculated parameters and the measured parameters. The lack of consistency in the absolute value of this ratio is again likely a result of the lack of knowledge about the specifics of the installation and the luminaire type. 


\subsubsection{Discussion}

The comparison of the measured and the calculated results is relatively poor, particularly in terms of the absolute values. The difficulty with all of these experiments is a lack of consistent documentation, unknown input factors to the calculation model, and unknown luminaire and lamp combinations.

However, the general expectations of the addition of reflected light to the target luminance calculation are verified by the comparisons. This is particularly evident in the Hendersonville experiment and the Scottsdale experiment.

In order to fully verify the reflection data and the calculation program, a new set of measurement experiments must be undertaken in the field. These experiments must be carefully controlled and performed. The expectation of the results, however, must be carefully considered. Previous research by Janoff [1993] has shown that even in the best of pavement luminance experiments, the results have not yielded what would typically be acceptable comparisons of calculated to measured results. This is due to variations in the pavement, geometry and luminaires used in the installation. With the addition of further reflection influences and the target variation, the comparison of the measured to the calculated parameters will never be very precise.

\subsection{Impact on Visibility Level}

The impact of the addition of reflected light to the visibility level calculation was 
established by calculating the visibility level on a series of sample installations first with and then without reflected light. The sample installations were comprised of opposite, staggered and single-sided luminaire arrangements. Opposite arrangements have two luminaires located on either side of the road at the same point along the length of the roadway. A single-sided installation is one in which the luminaires are all located along one side of the roadway at a given spacing. A staggered installation refers to an arrangement where lamps are placed along the roadway on alternating sides of the street. The GE7320.ies luminaire intensity distribution file was used in this calculation. Eight luminaires were used in the calculation and spaced at either 71 metre spacing or 132 metre spacing depending on the arrangement. The calculation grid used in this test was eight rows across the 22 metre roadway by 22 rows along the roadway. The calculation grid was located from 60 metres to 160 metres from the observer.

The results of the visibility calculation are shown in Tables 35,37 and 39 for the direct calculations, and Tables 36,38 and 40 for the direct and indirect calculations. The impact of the reflected light on the individual measurements of target luminance, contrast and visibility level are also shown in Figures 103, 105 and 107. The impact of the reflected light on the target contrast and the visibility level is shown in Figures 104, 106 and 108 for the three arrangements calculated as the difference of the calculation with reflected light and the calculation without reflected light. It should be noted that these test arrangements are examples only and do not meet the criteria of IESNA [1997] as high quality lighting designs. 
Table 35 - Visibility Results for Single-Sided Arrangement without Indirect Target Luminance

\begin{tabular}{||r|r|r|r|r|r|r|r|r|}
\hline \multicolumn{70}{|c|}{ Visibility Distribution } \\
\hline Grid Dist. & \multicolumn{7}{|c|}{ Across } \\
\hline Long. & 0 & 3.14 & 6.29 & 9.43 & 12.57 & 15.71 & 18.86 & 22 \\
\hline 160 & 2.19 & 3.12 & 0.77 & 0.79 & 1.08 & 0.81 & 0.51 & 0.27 \\
\hline 155.24 & 1.79 & 2.61 & 0.03 & 1.56 & 1.37 & 0.92 & 0.52 & 0.25 \\
\hline 150.48 & 0.29 & 0.86 & 1.49 & 1.45 & 1.1 & 0.7 & 0.3 & 0.11 \\
\hline 145.71 & 1.22 & 1.3 & 2.33 & 1.2 & 0.53 & 0.25 & 0.07 & 0.03 \\
\hline 140.95 & 1.34 & 2.13 & 2.02 & 0.75 & 0.04 & 0.11 & 0.03 & 0.01 \\
\hline 136.19 & 0.72 & 0.63 & 0.88 & 0.85 & 0.7 & 0.41 & 0.21 & 0.09 \\
\hline 131.43 & 2.88 & 4.97 & 3.79 & 2.44 & 1.48 & 0.86 & 0.37 & 0.16 \\
\hline 126.67 & 3.34 & 5.28 & 4.22 & 2.56 & 1.36 & 0.71 & 0.35 & 0.18 \\
\hline 121.9 & 4.66 & 6.83 & 5.63 & 3.01 & 1.28 & 0.64 & 0.31 & 0.16 \\
\hline 117.14 & 5.37 & 7.82 & 5.59 & 3.01 & 1.63 & 0.75 & 0.27 & 0.07 \\
\hline 112.38 & 5.95 & 8.53 & 6.29 & 3.46 & 1.86 & 0.88 & 0.28 & 0.03 \\
\hline 107.62 & 6.23 & 8.94 & 6.36 & 3.42 & 1.64 & 0.68 & 0.17 & 0.08 \\
\hline 102.86 & 6.34 & 8.69 & 5.44 & 2.54 & 1.01 & 0.07 & 0.29 & 0.34 \\
\hline 98.1 & 5.9 & 8.17 & 4.34 & 0.97 & 0.45 & 0.77 & 0.79 & 0.48 \\
\hline 93.33 & 3.32 & 5.16 & 0.76 & 1.49 & 2.23 & 1.61 & 0.94 & 0.5 \\
\hline 88.57 & 0.63 & 0.22 & 4.7 & 4.67 & 2.83 & 1.58 & 0.78 & 0.3 \\
\hline 83.81 & 3.61 & 4.47 & 6.03 & 3.53 & 1.61 & 0.76 & 0.22 & 0.04 \\
\hline 79.05 & 3.38 & 4.2 & 4.54 & 1.85 & 0.22 & 0.05 & 0.07 & 0.02 \\
\hline 74.29 & 0.8 & 0.35 & 0.04 & 0.91 & 1.06 & 0.46 & 0.15 & 0.07 \\
\hline 69.52 & 5.99 & 8.16 & 7.2 & 4.79 & 2.73 & 1.56 & 0.83 & 0.47 \\
\hline 64.76 & 9.39 & 14.73 & 12.82 & 7.81 & 4.53 & 2.88 & 1.59 & 0.79 \\
\hline 60 & 12.36 & 18.03 & 14.72 & 9.18 & 5.21 & 2.97 & 1.58 & 0.84 \\
\hline & & & & & & & \\
\hline
\end{tabular}


Table 36 - Visibility Results for Single-Sided Arrangement with Indirect Target Luminance

\begin{tabular}{|c|c|c|c|c|c|c|c|c|}
\hline \multicolumn{9}{|c|}{ Visibility Distribution } \\
\hline Grid Dist. & \multicolumn{8}{|c|}{ Across } \\
\hline Long. & 0 & 3.14 & 6.29 & 9.43 & 12.57 & 15.71 & 18.86 & 22 \\
\hline 160 & 1.74 & 2.54 & 0.12 & 1.87 & 1.95 & 1.39 & 0.86 & 0.47 \\
\hline 155.24 & 1.23 & 1.91 & 1.13 & 2.61 & 2.13 & 1.4 & 0.8 & 0.4 \\
\hline 150.48 & 0.34 & 0.14 & 2.42 & 2.16 & 1.6 & 1.03 & 0.49 & 0.21 \\
\hline 145.71 & 1.87 & 2.14 & 3.14 & 1.77 & 0.88 & 0.47 & 0.21 & 0.12 \\
\hline 140.95 & 1.87 & 2.9 & 2.75 & 1.22 & 0.22 & 0.06 & 0.11 & 0.12 \\
\hline 136.19 & 0.35 & 0.04 & 0.36 & 0.5 & 0.46 & 0.23 & 0.08 & 0 \\
\hline 131.43 & 2.55 & 4.42 & 3.3 & 2.1 & 1.25 & 0.68 & 0.25 & 0.08 \\
\hline 126.67 & 3.02 & 4.87 & 3.75 & 2.21 & 1.1 & 0.52 & 0.21 & 0.08 \\
\hline 121.9 & 4.34 & 6.45 & 5.22 & 2.62 & 0.99 & 0.42 & 0.15 & 0.04 \\
\hline 117.14 & 5.09 & 7.53 & 5.21 & 2.59 & 1.26 & 0.45 & 0.06 & 0.1 \\
\hline 112.38 & 5.68 & 8.26 & 5.88 & 2.96 & 1.36 & 0.46 & 0.05 & 0.25 \\
\hline 107.62 & 5.95 & 8.66 & 5.89 & 2.76 & 0.92 & 0.04 & 0.41 & 0.5 \\
\hline 102.86 & 5.95 & 8.25 & 4.67 & 1.49 & 0.09 & 1.08 & 1.15 & 0.9 \\
\hline 98.1 & 5.21 & 7.34 & 2.97 & 0.91 & 2.39 & 2.26 & 1.77 & 1.07 \\
\hline 93.33 & 2.05 & 3.57 & 1.81 & 4.14 & 4.33 & 3.03 & 1.82 & 1.03 \\
\hline 88.57 & 2.75 & 3.01 & 7.74 & 7.13 & 4.53 & 2.68 & 1.44 & 0.67 \\
\hline 83.81 & 5.6 & 7.09 & 8.43 & 5.22 & 2.71 & 1.48 & 0.66 & 0.3 \\
\hline 79.05 & 5.07 & 6.49 & 6.66 & 3.23 & 1.07 & 0.48 & 0.27 & 0.23 \\
\hline 74.29 & 0.19 & 1.29 & 1.57 & 0.02 & 0.48 & 0.04 & 0.21 & 0.2 \\
\hline 69.52 & 5.14 & 6.84 & 6 & 3.93 & 2.12 & 1.1 & 0.52 & 0.25 \\
\hline 64.76 & 8.56 & 13.66 & 11.76 & 6.95 & 3.91 & 2.39 & 1.23 & 0.53 \\
\hline 60 & 11.55 & 17.15 & 13.6 & 8.24 & 4.54 & 2.44 & 1.16 & 0.52 \\
\hline
\end{tabular}




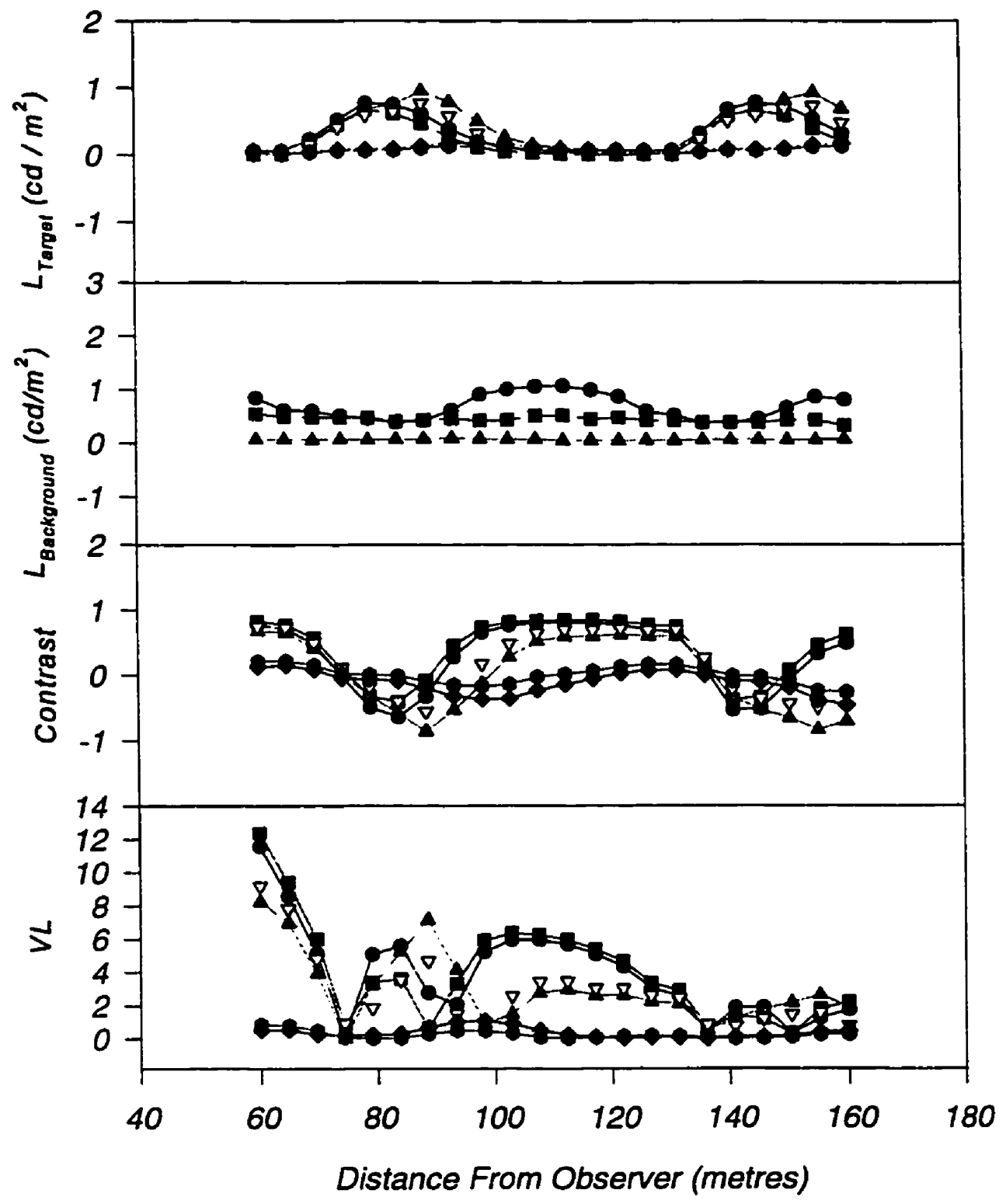

With Reflected Light

$\begin{array}{ll}- & R o w 1 \\ - & R o w \\ - & R o w \\ 8\end{array}$

Without Reflected Light

- Row 1

$\nabla$ Row 4

- Row 8

Figure 103 - Impact of Reflected Light for Single-Sided Arrangement 


\section{NOTE TO USERS}

Page(s) not included in the original manuscript are unavailable from the author or university. The manuscript was microfilmed as received.

\section{UMI}


Table 37 - Visibility Results for Opposite Arrangement with Indirect Target Luminance

\begin{tabular}{|c|c|c|c|c|c|c|c|c|}
\hline \multicolumn{9}{|c|}{ Visibility Distribution } \\
\hline Grid Dist. & \multicolumn{8}{|c|}{ Across } \\
\hline Long. & 0 & 3.14 & 6.29 & 9.43 & 12.57 & 15.71 & 18.86 & 22 \\
\hline 160 & 2.53 & 4.2 & 5.75 & 6.48 & 6.15 & 4.73 & 3.1 & 1.55 \\
\hline 155.24 & 3.58 & 5.65 & 7.33 & 7.94 & 7.53 & 6.25 & 4.33 & 2.16 \\
\hline 150.48 & 4.13 & 6.15 & 7.03 & 6.64 & 6.87 & 6.63 & 4.87 & 2.54 \\
\hline 145.71 & 3.9 & 5.48 & 5.65 & 4.5 & 5.27 & 5.82 & 4.52 & 2.54 \\
\hline 140.95 & 2.72 & 4.02 & 3.37 & 2.19 & 2.84 & 4.73 & 3.43 & 1.71 \\
\hline 136.19 & 0.28 & 0.09 & 0.71 & 1.02 & 0.72 & 0.01 & 0.6 & 0.24 \\
\hline 131.43 & 2.83 & 4.77 & 4.12 & 3.62 & 3.89 & 4.43 & 3.29 & 1.47 \\
\hline 126.67 & 3.42 & 5.23 & 4.53 & 4 & 4.35 & 4.89 & 3.64 & 1.93 \\
\hline 121.9 & 4.72 & 6.78 & 5.85 & 4.66 & 5.58 & 6.42 & 5.25 & 2.74 \\
\hline 117.14 & 5.5 & 7.92 & 6.05 & 4.86 & 5.91 & 7.07 & 6.11 & 3.07 \\
\hline 112.38 & 6.18 & 8.73 & 6.87 & 5.65 & 6.81 & 8.1 & 6.68 & 3.29 \\
\hline 107.62 & 6.61 & 9.27 & 7.22 & 6.28 & 7.72 & 8.65 & 6.45 & 2.87 \\
\hline 102.86 & 6.9 & 9.3 & 6.92 & 6.01 & 7.57 & 8.94 & 5.75 & 2.04 \\
\hline 98.1 & 6.92 & 9.42 & 7.04 & 6.29 & 8 & 9.08 & 4.86 & 1.36 \\
\hline 93.33 & 5.52 & 8.19 & 6.17 & 5.87 & 6.94 & 7.96 & 3.56 & 0.92 \\
\hline 88.57 & 3.63 & 5.73 & 4.42 & 4.2 & 4.87 & 5.56 & 2.42 & 0.6 \\
\hline 83.81 & 2.07 & 3.36 & 2.35 & 2.05 & 2.48 & 3.36 & 1.42 & 0.35 \\
\hline 79.05 & 1.26 & 2.07 & 1.04 & 0.92 & 1.21 & 2.1 & 0.89 & 0.23 \\
\hline 74.29 & 0.81 & 1.23 & 0.48 & 0.38 & 0.59 & 1.26 & 0.54 & 0.1 \\
\hline 69.52 & 0.51 & 0.78 & 0.22 & 0.16 & 0.31 & 0.8 & 0.32 & 0.01 \\
\hline 64.76 & 0.27 & 0.46 & 0.06 & 0.08 & 0.09 & 0.48 & 0.14 & 0.14 \\
\hline 60 & 0.06 & 0.21 & 0.15 & 0.3 & 0.13 & 0.22 & 0.06 & 0.3 \\
\hline
\end{tabular}


Table 38 - Visibility Results for Opposite Arrangement without Indirect Target Luminance

\begin{tabular}{|c|c|c|c|c|c|c|c|c|}
\hline \multicolumn{9}{|c|}{ Visibility Distribution } \\
\hline \multirow{2}{*}{$\begin{array}{l}\text { Grid Dist. } \\
\text { Long. }\end{array}$} & \multicolumn{8}{|c|}{ Across } \\
\hline & 0 & 3.14 & 6.29 & 9.43 & 12.57 & 15.71 & 18.86 & 22 \\
\hline 160 & 1.47 & 2.48 & 3.61 & 4.18 & 3.93 & 2.85 & 1.8 & 0.93 \\
\hline 155.24 & 2.38 & 3.81 & 5.25 & 5.82 & 5.44 & 4.3 & 2.87 & 1.44 \\
\hline 150.48 & 3.08 & 4.68 & 5.54 & 5.27 & 5.44 & 5.13 & 3.64 & 1.9 \\
\hline 145.71 & 3.07 & 4.36 & 4.56 & 3.55 & 4.24 & 4.68 & 3.55 & 1.99 \\
\hline 140.95 & 2.09 & 3.13 & 2.53 & 1.49 & 2.06 & 3.79 & 2.69 & 1.27 \\
\hline 136.19 & 0.7 & 0.57 & 1.29 & 1.5 & 1.27 & 0.67 & 0.03 & 0.12 \\
\hline 131.43 & 3.19 & 5.33 & 4.63 & 4.04 & 4.35 & 5 & 3.78 & 1.76 \\
\hline 126.67 & 3.76 & 5.65 & 5.01 & 4.42 & 4.8 & 5.35 & 4.04 & 2.22 \\
\hline 121.9 & 5.03 & 7.14 & 6.26 & 5.08 & 5.98 & 6.8 & 5.59 & 2.99 \\
\hline 117.14 & 5.74 & 8.18 & 6.41 & 5.26 & 6.27 & 7.37 & 6.37 & 3.28 \\
\hline 112.38 & 6.38 & 8.95 & 7.21 & 6.05 & 7.16 & 8.36 & 6.9 & 3.47 \\
\hline 107.62 & 6.77 & 9.43 & 7.49 & 6.61 & 7.98 & 8.84 & 6.62 & 3.03 \\
\hline 102.86 & 7.01 & 9.41 & 7.11 & 6.25 & 7.75 & 9.07 & 5.89 & 2.19 \\
\hline 98.1 & 7.02 & 9.51 & 7.16 & 6.43 & 8.12 & 9.17 & 4.99 & 1.49 \\
\hline 93.33 & 5.62 & 8.28 & 6.29 & 5.99 & 7.05 & 8.05 & 3.69 & 1.04 \\
\hline 88.57 & 3.76 & 5.84 & 4.56 & 4.34 & 5 & 5.67 & 2.56 & 0.71 \\
\hline 83.81 & 2.23 & 3.51 & 2.51 & 2.22 & 2.65 & 3.51 & 1.57 & 0.46 \\
\hline 79.05 & 1.43 & 2.26 & 1.22 & 1.1 & 1.39 & 2.28 & 1.05 & 0.35 \\
\hline 74.29 & 1.01 & 1.45 & $\begin{array}{ll}0.68 \\
\end{array}$ & 0.56 & 0.79 & 1.48 & 0.72 & 0.23 \\
\hline 69.52 & 0.75 & 1.05 & 0.45 & 0.38 & 0.54 & 1.07 & 0.54 & 0.16 \\
\hline 64.76 & 0.57 & 0.79 & 0.34 & 0.2 & 0.38 & 0.8 & 0.41 & 0.08 \\
\hline 60 & 0.45 & 0.64 & 0.28 & 0.15 & 0.3 & 0.64 & 0.31 & 0.04 \\
\hline
\end{tabular}




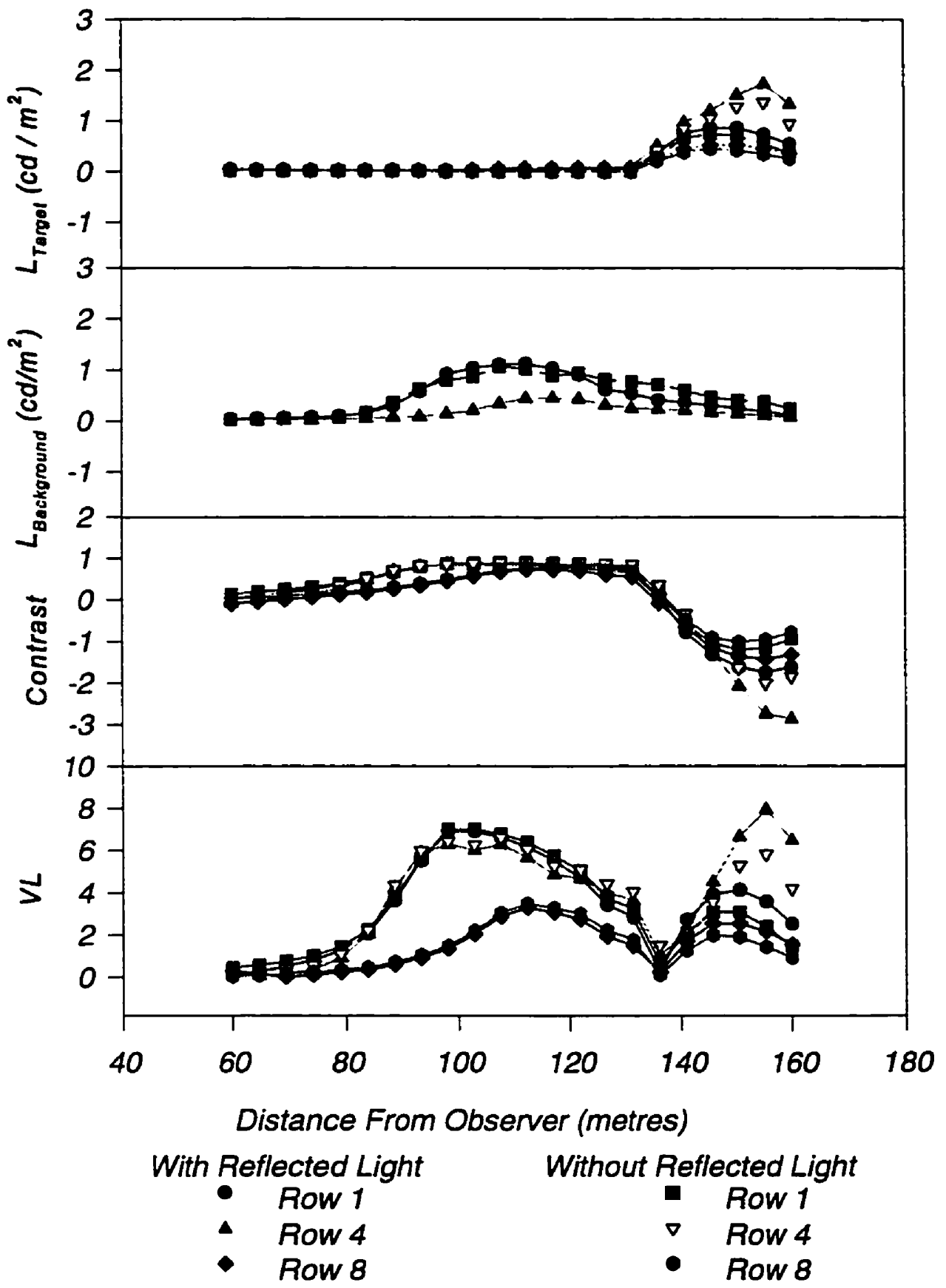

Figure 105 - Impact of Reflected Light for Opposite Arrangement 


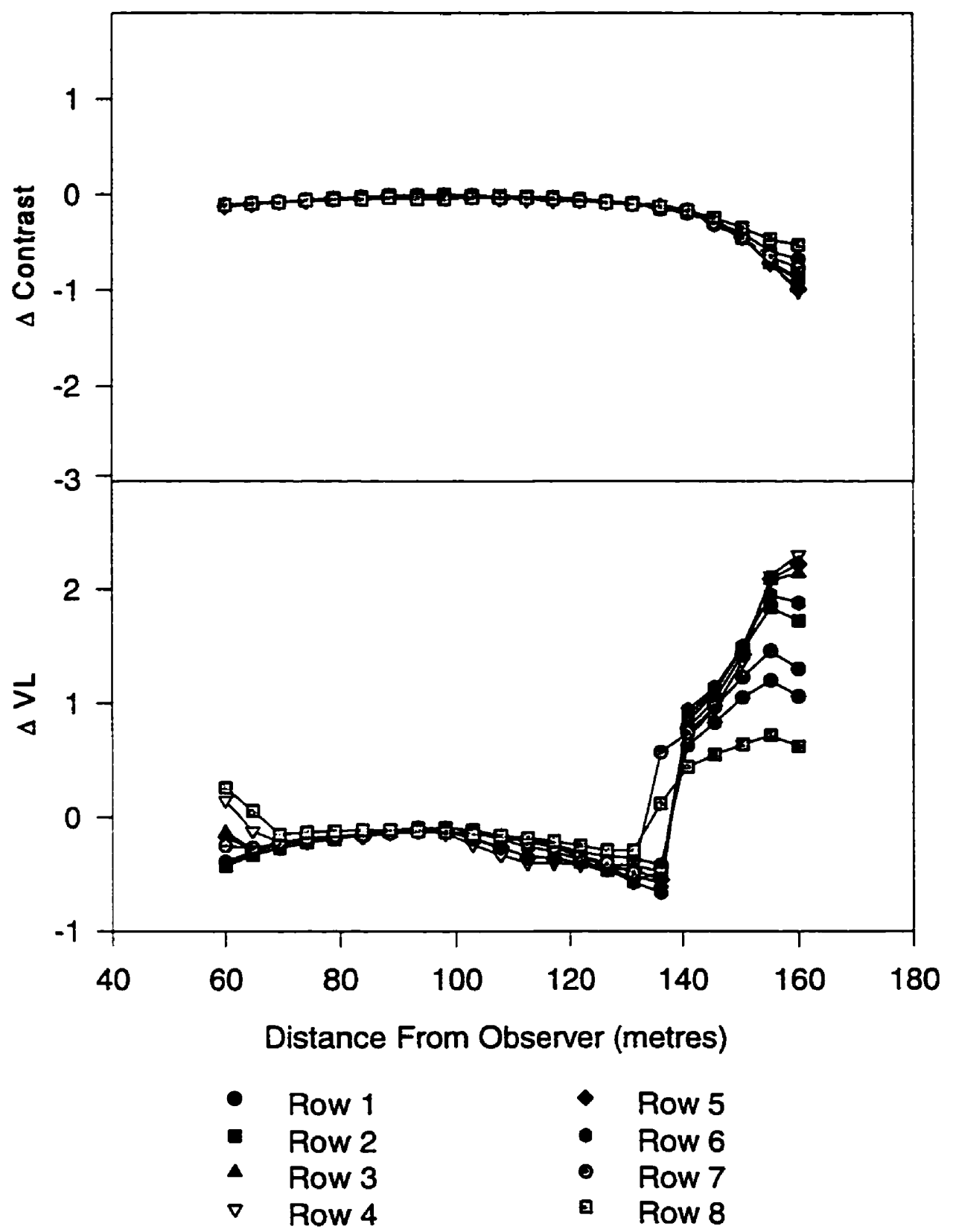

Figure 106 - Difference of Reflected and Non-Reflected Calculations for Target Contrast and Visibility Level for Opposite Arrangement 
Table 39 - Visibility Results for Staggered Arrangement with Indirect Target Luminance

\begin{tabular}{||r|r|r|r|r|r|r|r|r|}
\hline \multicolumn{8}{|c|}{ Visibility Distribution } \\
\hline Grid Dist. & \multicolumn{7}{|c|}{ Across } \\
\hline Long. & 0 & 3.14 & 6.29 & 9.43 & 12.57 & 15.71 & 18.86 & 23 \\
\hline 160 & 2.75 & 1.43 & 0.1 & 1.2 & 2.91 & 3.85 & 1.73 & 0.36 \\
\hline 155.24 & 4.57 & 2.69 & 0.51 & 1.38 & 3.22 & 4.12 & 1.68 & 0.2 \\
\hline 150.48 & 5.51 & 3.66 & 1.05 & 1 & 2.49 & 3.43 & 1.06 & 0.08 \\
\hline 145.71 & 5.38 & 3.83 & 1.5 & 0.19 & 1.29 & 2.01 & 0.56 & 0.01 \\
\hline 140.95 & 4.54 & 3.01 & 1.14 & 0.61 & 0.1 & 0.9 & 0.17 & 0.03 \\
\hline 136.19 & 0.04 & 0.25 & 0.03 & 0.04 & 0.19 & 0.6 & 0.14 & 0.02 \\
\hline 131.43 & 4.39 & 3.34 & 1.53 & 1.11 & 0.79 & 0.82 & 0.3 & 0.06 \\
\hline 126.67 & 4.86 & 3.82 & 1.93 & 1.24 & 0.81 & 0.66 & 0.21 & 0.02 \\
\hline 121.9 & 6.5 & 5.29 & 2.68 & 1.61 & 0.88 & 0.52 & 0.13 & 0.04 \\
\hline 117.14 & 7.39 & 6.24 & 2.96 & 1.44 & 0.83 & 0.44 & 0.07 & 0.11 \\
\hline 112.38 & 8.31 & 6.86 & 3.21 & 1.33 & 0.69 & 0.28 & 0.08 & 0.27 \\
\hline 107.62 & 8.9 & 7.28 & 2.92 & 1.22 & 0.35 & 0.03 & 0.42 & 0.65 \\
\hline 102.86 & 8.69 & 7.5 & 2.51 & 0.5 & 0.07 & 0.59 & 1.19 & 1.75 \\
\hline 98.1 & 8.89 & 7.31 & 1.97 & 0.04 & 0.63 & 1.77 & 3.37 & 4.83 \\
\hline 93.33 & 7.52 & 5.82 & 0.91 & 0.34 & 1.56 & 4.29 & 7.63 & 10.08 \\
\hline 88.57 & 4.89 & 3.54 & 0.28 & 0.76 & 2.71 & 7.06 & 11.15 & 13.43 \\
\hline 83.81 & 2.5 & 1.75 & 0.12 & 1.09 & 2.95 & 7.63 & 10.6 & 11.54 \\
\hline 79.05 & 1.41 & 1.03 & 0.66 & 1.76 & 2.45 & 4.97 & 8.18 & 8.48 \\
\hline 74.29 & 1 & 0.74 & 0.4 & 0.93 & 0.19 & 0.88 & 2.3 & 4.11 \\
\hline 69.52 & 1.18 & 1.6 & 1.65 & 2.41 & 3.74 & 6.69 & 5.4 & 4.83 \\
\hline 64.76 & 1.62 & 2.71 & 3.97 & 5.28 & 6.62 & 10.26 & 13.27 & 10.34 \\
\hline 60 & 1.64 & 2.97 & 4.27 & 6.3 & 8.12 & 14 & 15.56 & 12.03 \\
\hline \hline
\end{tabular}


Table 40 - Visibility Results for Staggered Arrangement without Indirect Target Luminance

\begin{tabular}{|c|c|c|c|c|c|c|c|c|}
\hline \multicolumn{9}{|c|}{ Visibility Distribution } \\
\hline Grid Dist. & \multicolumn{8}{|c|}{ Across } \\
\hline Long. & 0 & 3.14 & 6.29 & 9.43 & 12.57 & 15.71 & 18.86 & 22 \\
\hline 160 & 1.37 & 0.47 & 0.33 & 1.38 & 2.99 & 3.9 & 1.79 & 0.43 \\
\hline 155.24 & 2.93 & 1.49 & 0.06 & 1.57 & 3.3 & 4.17 & 1.74 & 0.25 \\
\hline 150.48 & 4.14 & 2.58 & 0.52 & 1.19 & 2.58 & 3.49 & 1.14 & 0.13 \\
\hline 145.71 & 4.29 & 2.94 & 1.02 & 0.41 & 1.43 & 2.1 & 0.64 & 0.06 \\
\hline 140.95 & 3.65 & 2.33 & 0.75 & 0.32 & 0.29 & 1.03 & 0.27 & 0.03 \\
\hline 136.19 & 0.59 & 0.27 & 0.28 & 0.22 & 0.4 & 0.76 & 0.25 & 0.05 \\
\hline 131.43 & 4.94 & 3.77 & 1.82 & 1.36 & 0.98 & 0.99 & 0.42 & 0.13 \\
\hline 126.67 & 5.29 & 4.17 & 2.21 & 1.48 & 1.01 & 0.82 & 0.33 & 0.1 \\
\hline 121.9 & 6.85 & 5.61 & 2.93 & 1.83 & 1.06 & 0.68 & 0.25 & 0.06 \\
\hline 117.14 & 7.65 & 6.48 & 3.17 & 1.61 & $i$ & 0.6 & 0.23 & 0.04 \\
\hline 112.38 & 8.52 & 7.05 & 3.4 & 1.5 & 0.87 & 0.49 & 0.16 & 0.01 \\
\hline 107.62 & 9.05 & 7.42 & 3.1 & 1.41 & 0.57 & 0.26 & 0.01 & 0.19 \\
\hline 102.86 & 8.8 & 7.61 & 2.69 & 0.72 & 0.23 & 0.01 & 0.3 & 0.67 \\
\hline 98.1 & 8.98 & 7.42 & 2.17 & 0.3 & 0.08 & 0.6 & 1.39 & 2.38 \\
\hline 93.33 & 7.62 & 5.95 & 1.13 & 0.03 & 0.71 & 2.21 & 4.16 & 6.19 \\
\hline 88.57 & 5.03 & 3.71 & 0.51 & 0.34 & 1.66 & 4.55 & 7.39 & 9.65 \\
\hline 83.81 & 2.68 & 1.96 & 0.15 & 0.62 & 1.96 & 5.49 & 7.68 & 8.84 \\
\hline 79.05 & 1.62 & 1.28 & 0.21 & 1.09 & 1.47 & 3.31 & 5.77 & 6.27 \\
\hline 74.29 & 1.25 & 1.06 & 0.14 & 0.17 & 0.86 & 1.76 & 0.55 & 2.39 \\
\hline 69.52 & 1.48 & 2 & 2.16 & 3.01 & 4.41 & 7.42 & 6.77 & $6.0^{7}$ \\
\hline 64.76 & 1.96 & 3.13 & 4.47 & 5.85 & 7.26 & 10.92 & 14.24 & 11.4 \\
\hline 60 & 2.02 & 3.4 & 4.77 & 6.85 & 8.73 & 14.58 & 16.43 & 13.15 \\
\hline
\end{tabular}




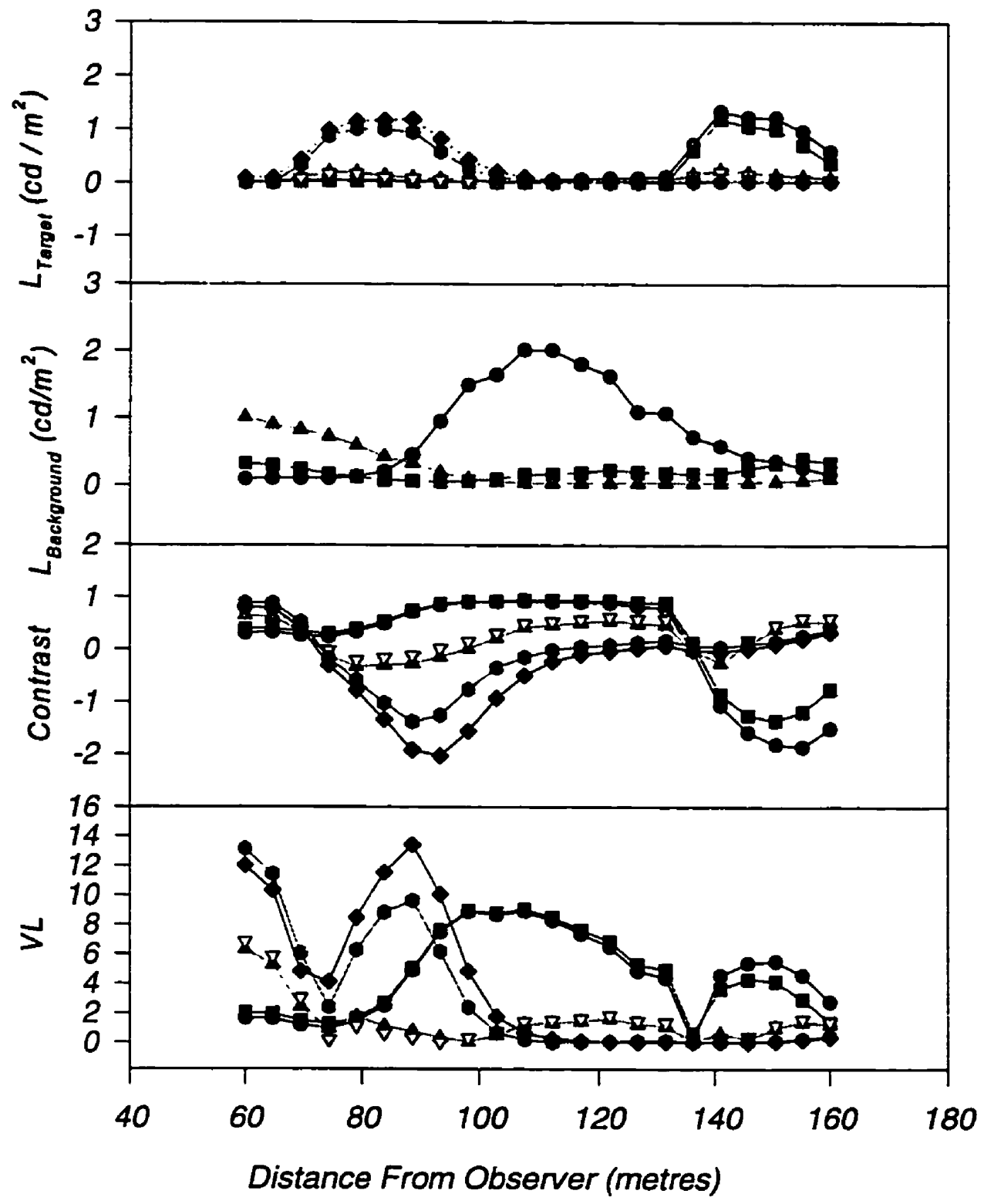

With Reflected Light

- Row 1

- Row 4

- Row 8

Without Reflected Light

- Row 1

$\nabla$ Row 4

- Row 8

Figure 107 - Impact of Reflected Light for Staggered Arrangement 


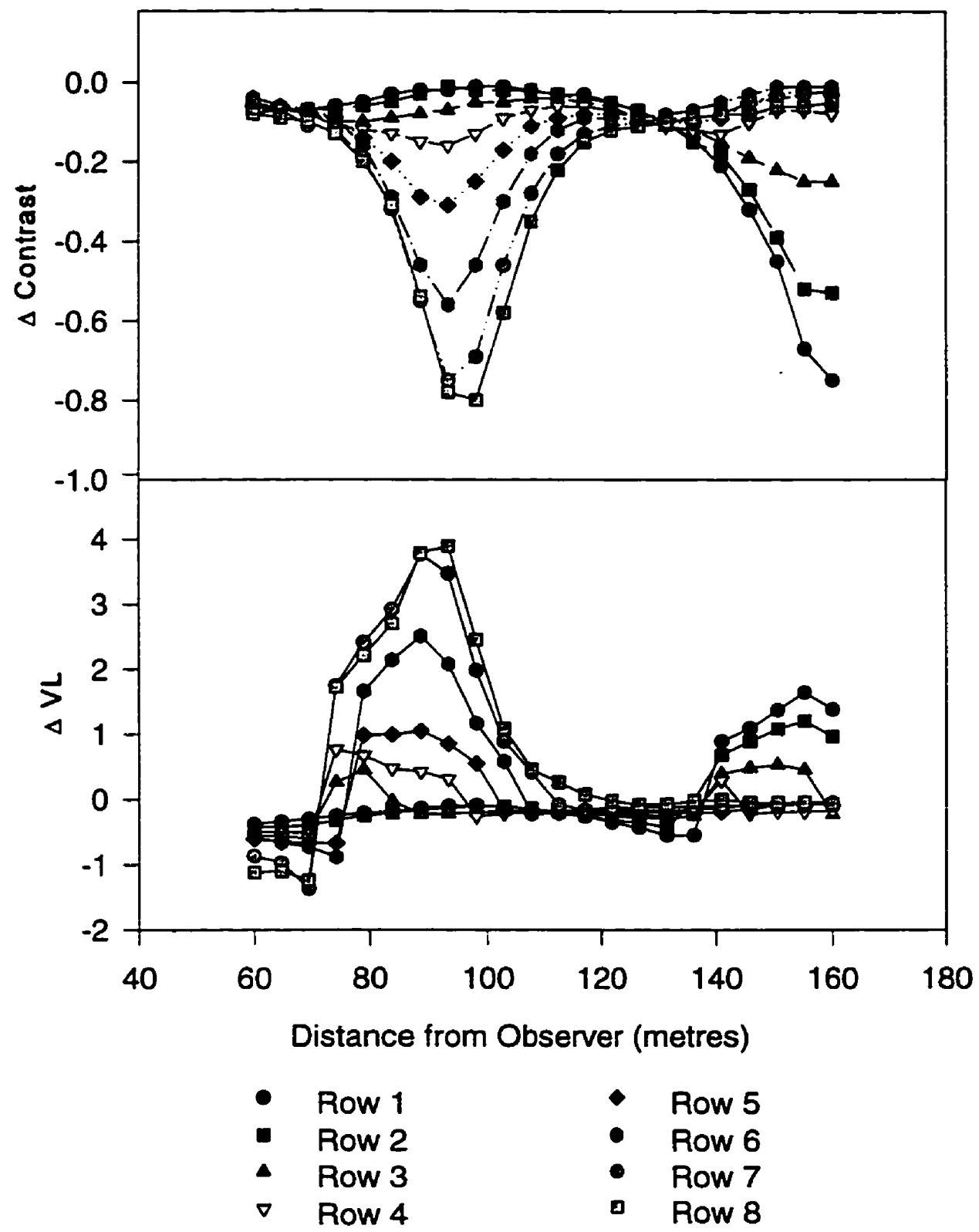

Figure 108 - Difference of Reflected and Non-Reflected Calculations for Target Contrast and Visibility Level for Staggered Arrangement 
The addition of the reflected light to the target luminance did impact the results as expected. The reflected light increased positive contast, which means that dark targets on a bright background are less easily seen. For negative contrast targets, bright targets on a dark background, the addition of the reflected light allowed the target to be seen more easily. These results are seen in Figures 104, 106 and 108. This affect on contrast can explain why, when comparing measured to calculated results, for individual targets the contrast can be reversed.

The impact of the addition of the reflected portion on the overall weighted visibility level is shown in Table 41.

Table 41 - Weighted Visibility Level Results for Different Luminaire Arrangements and Calculation Methods

\begin{tabular}{||l|r|r|}
\hline Weighted VL & Reflection & No Reflection \\
\hline Staggered & 2.17 & 2.06 \\
\hline Opposite & 3.01 & 2.98 \\
\hline Single Sided & 1.95 & 1.80 \\
\hline \hline & \multicolumn{3}{|c|}{} \\
\hline \hline Minimum & Reflection & No Reflection \\
\hline Staggered & 0.01 & 0.01 \\
\hline Opposite & 0.01 & 0.03 \\
\hline Single Sided & 0.00 & 0.01 \\
\hline \hline \multicolumn{3}{|c|}{} \\
\hline \hline Maximum & Reflection & No Reflection \\
\hline Staggered & 15.56 & 16.43 \\
\hline Opposite & 9.42 & 9.51 \\
\hline Single Sided & 17.15 & 18.03 \\
\hline \hline
\end{tabular}


The results for the weighted average showed very little change in the weighted visibility level. The maximum and the minimum visibility levels were also included in the table. The maximum visibility level does show an effect with the addition of the reflected light. The addition of reflected light seems to be far more related to the specific position of the target in the design field than with the average visibility level of the roadway. The maximum and the minimum values of visibility level are related to a specific point on the road surface where the average is an overall impact of the lighting design.

\subsection{Conclusions}

A system has been developed for calculating the indirect illuminance of an object in the roadway. Based on this system, calculations were made which allowed the determination of the target visibility.

The indirect target illuminance is calculated based on the sum of the light reflected from a series of pavement areas in front of the target. These areas, which comprise an integration area, are based on the size of the target. By calculating the target illuminance for different sizes and shapes of the reflection integration area, it was found that the maximum target illuminance was found with a square integration area, with 20 by 20 calculation areas, each calculation area being nine times the area of the target.

Using the system developed, calculated results were compared to experimental results. The absolute results were not comparable, but in many cases the relative results and the 
expected results were the same. In order to fully verify the calculated results, a more carefully designed experiment must be conducted which uses precise geometry and carefully photometered luminaires and lamps. The expectation of such an experiment must be carefully considered. There are many factors which affect the measured results of a lighting installation. Large deviations between calculated and measured results will likely always be evident in these comparisons.

The impact of the reflected light on the weighted average visibility level is relatively small while the impact of the reflected light from a pavement surface on individual points in the calculation grid is far greater. The addition of reflected light into the visibility level calculation, although having a small impact on the overall lighting design quality criterion, does represent the real world installation more closely and should continue to be used. 


\section{Conclusions}

This research into the influence of pavement reflection on target visibility has been carried out in four stages. The first established the reflection properties of pavements for angles greater than one degree. The second stage studied the surface roughness of the pavements and tried to relate these results to the reflection properties. The third considered an analytical model of the reflection data. The fourth studied the impact of the reflection data on the visibility level for roadway lighting design.

The reflection profiles of 20 pavement samples ranging from highly specular to diffuse was measured on a gonioreflectometer. As expected, it was determined that the reflection profile was dependent on the angles of observation and incidence. It was also found that the reflection was dependent on the pavement type and the R-Class. The reflection results were correlated to the classification parameters for pavements which are currently used by the CIE. The older CIE system and the proposed CIE system were also considered but these systems added no new information to the reflection profile data. A new system based on the roughness of the sample was also examined and found to be consistent with the expected results. The most important aspect of the reflection profile was found to be the trend towards Lambertian reflection as the observation angle increased.

The surface roughness of the same 20 pavement samples was established using a stylus system which allowed for the measurement of the surface at several points across the 
sample. The correlation of the roughness to the reflection characteristics was carried out and no significant correlations were found. As expected, the roughness was correlated with the sample recipe. The roughness results were also compared with the CIE reflection parameter classifications and no significant relationship was found.

Two methodologies were considered for analytically modeling the reflection data. Both systems showed correlation with the reflection data but a complete model was not able to be developed. A complete model requires further measurement of the pavement sample surface to more accurately define macro and micro roughness and to find the orientation of the aggregate in the surface.

A calculation method for the indirect illuminance was determined using iterative calculations maximizing the target illuminance. The calculation results were also compared to the results of investigations which have been carried out in actual lighting installations. The correlation of the absolute results of the calculations to the measured results was not very high but the comparison of the contributions of the reflected light was verified. Further experiments to verify these results are required.

The impact of the reflected light on the visibility level was assessed through the calculation of the visibility level with and without reflected light. It was found that the impact of the reflected light on the total weighted visibility level was not very significant. However, the impact of the visibility level for individual points in the 
calculation may be significant depending on the position of the target in the measurement field.

The introduction of the visibility level in the current revision of the IESNA RP-8 has been delayed due to the inability of the calculated results to be verified in actual installations. The purpose of this research was to establish a system to account for one of the missing pieces in the calculation of the visibility level. This has been accomplished. A calculation system for the target visibility has been established and verified. Although the results do not explain wide variations in the visibility level, they do aid in explaining the differences found for specific points in the measurement field. Hopefully, the verification of the RP-8 can now continue and the visibility level can be introduced as the quality criterion for roadway lighting design. 


\section{References}

1. Adrian, W.K., "Visibility of Targets: Model for Calculation", Lighting Research and Technology, 21(4) pp.181-188 (1989)

2. Adrian, W.K., and J. Enzmann, Die Berechnung der Leuchtdichte und Blendung in der Strassenbeleuchtung mit Hilfe von Elektronischen Rechenmaschin, Karlsruhe, 1971

3. Alcade, H. P.. P. A. B. Vidal, and P.R. Ixtaina, "New System for the Description of the Reflection Indicatrix of Road Surfaces", Laboratorio de Acustica y Luminotecnia, Comision de Investigaciones Cientificas de la Provincia de Buenos Aires, Gonnet, Argentina, 1996

4. Beckmann, P and A Spizzichino, The Scattering of Electromagnetic Waves from Rough Surfaces, Macmillan, New York, 1963

5. Box, P.C., "Relationship Between Dlumination and Freeway Accidents", Illuminating Engineering, 66(5), May/June 1971

6. Box, P. C., "Comparison of Accidents and Illumination", Highway Research Record, (416) pp. 10-20, 1973

7. Brusque, C., and V. Carta, "Comparative Study of Parameters Qo and Qd Describing Road Surface Lightness", Proceeding Lux Europa, pp 1051-1058, 1997

8. Commission Internationale d'Eclairage, Calculation of Road Luminance, CIE Report \#30-2 (1982), Vienna, Austria

9. Commission Internationale d'Eclairage, Road Surface and Road Marking Reflection Characteristics, Draft report of Technical Commitee TC 4-25, Vienna, Austria, Draft Date September 1995

10. Commission Internationale d'Eclairage, A Review of Publications on Properties and Reflection Values of Material Reflection Standards, CIE Report \#46, Paris, France, 1979

11. Dmitrevsky, S and M. Bassett, Database for the Classification of Reflection Properties of Highway Pavement Surfaces, Project 22704, Volumes 1 through 4, University of Toronto, Department of Electrical Engineering, 1983 
12. Gibbons, Ronald B. And W.K. Adrian, "Influence of Observation Angle on Road Surface Reflection Characteristics", Proceedings IESNA Annual Conference, August 1996, pp 1050-1082

13. Gibbons, Ronald B., W. K. Adrian, Reflection Properties of Pavement for Observation Angles Greater than $1^{\circ}$, Report for the U.S. Federal Highway Administration, DTFH 61-93-P-01429, Washington D.C., May, 1994

14. Iluminating Engineering Society of North America, IES Lighting Handbook [1984], Reference Volume, New York, NY, 1984

15. Iluminating Engineering Society of North America, RP-8 (1997) - Proposed American National Standard Practice for Roadway Lighting Design, New York, NY, Draft Date 13 February 1997

16. International Standards Organization, Guide to the Expression of Uncertainty in Measurement, ISO/ TAG 4/ WG 3: June 1992

17. Janoff, M. S., B. Koth, W. McCunney, M. Freedman, C. Duerk, and M. Berkovitz, Effectiveness of Highway Arterial Lighting, Report for the U. S. Federal Highway Adminsitration, No. FHWA-RD-77-37, Washington D.C., 1977

18. Janoff, M. S.,"Measured vs. Computer Predicted Luminances in Roadway Lighting Applications", Journal of the IESNA, vol 22, No 1, Winter 1993, pp 75-82

19. Janoff, M. S., "The Relationship between Small Target Visibility and a Dynamic Measure of Visual Performance", Proceedings of the IESNA Annual Conference 1991, IESNA, New York, 1991

20. Jung, F. W., A. I. Titishov, and A. Kazakov, Road Surface Reflectance in Ontario, Ontario Ministry of Transportation and Communications, Province of Ontario, Downsview Ontario, 1984

21. Kebschull, W., "Die Reflexion trockner und feuchter Strassenbelage", Thesis, Berlin 1969.

22. Keck, M, Report on the Activities of the Visibility Task Force of the Roadway Lighting Committe, Roadway Lighting Committe, IESNA, June 1992

23. Ketvirtis A., and J. A. Bastianpillai, Road Surface Reflectance, Report for Road and Vehicle Traffic Safety Branch, Transport Canada, 1978 
24. King, E., “A Subjective Evaluation of Four Small Targets", Proceedings IESNA Annual Conference, Illuminating Engineering Society, New York, New York, Paper \# 28, (Preview Copy Dated February 1997)

25. Lecocq, Jacques, "The Application of the Visibility Level to Hemispherical Mutlifaceted Targets", Proceedings of the 2nd International Symposium on Visibility and Luminance in Roadway Lighting, Lighting Research Institute, New York, NY, 1993, pp 101-109

26. Lecocq, Jacques, "Visibility in Road Lighting, Correlation of Subjective Assessments with Calculated Values", Proceedings of Lux Europa, 1997, pp $22-36$

27. Mace, D., The Last Resource Inc, Safety Benefits of Roadway Lighting Using Small Target Visibility (STV) Design, Federal Highway Administration, Washington, D.C. 1997

28. Nieto-Vesperinas, M, Scattering and Diffraction in Physical Optics, John Wiley and Sons, New York, 1991

29. Schmidt-Clausen, H.J., W. Van Bommel, "Uber die Anwendung von linear polarisiertem Licht in der Kraftfahrzeugbeleuchtung", Tiel 1, Lichttechnik 1, 1972, pp 19-22

30. Scott, P.P., The Relationship Between Road Lighting Quality and Accident Frequency, Report No. LR929, Transportation and Road Research Laboratory, Great Britain, 1980

31. van Bommel, W. J. M. and J.B. deBoer, Road Lighting, Philips Technical Library, Kluwer, Antwerpen, 1980

32. Vermeulen, J., "Light Reflection from Rough Surfaces", Proceedings 18th Session of the CIE, Commission Internationale de L'Eclairage, Vienna, Austria, 1975, pp 569-576

33. Waldram, J.M., "Road Surface Reflection Characteristics and their Influence on Street Lighting Practice", Eleventh Annual Meeting of the Association of Public Lighting Engineers, Aberdeen England, 1934

34. Westermann, H.O., "Reflexionskennwerte von Strassenbelagen", Lichttechnik, vol 15, 1963, p. 507

35. White, P, "The Development of a Road Reflectivity Data Model", 4th Year 
Systems Design Workshop, Systems Design Engineering, University of

Waterloo, 1994 


\section{Appendix A}

The visibility level is illustrated in the following set of figures. A line of targets has been placed in the roadway beside the vehicle. The visibility of the lighting design would be determined based on the visibility of all the targets placed on the road. This target arrangement and the visibility of the targets is shown in Figure 109.

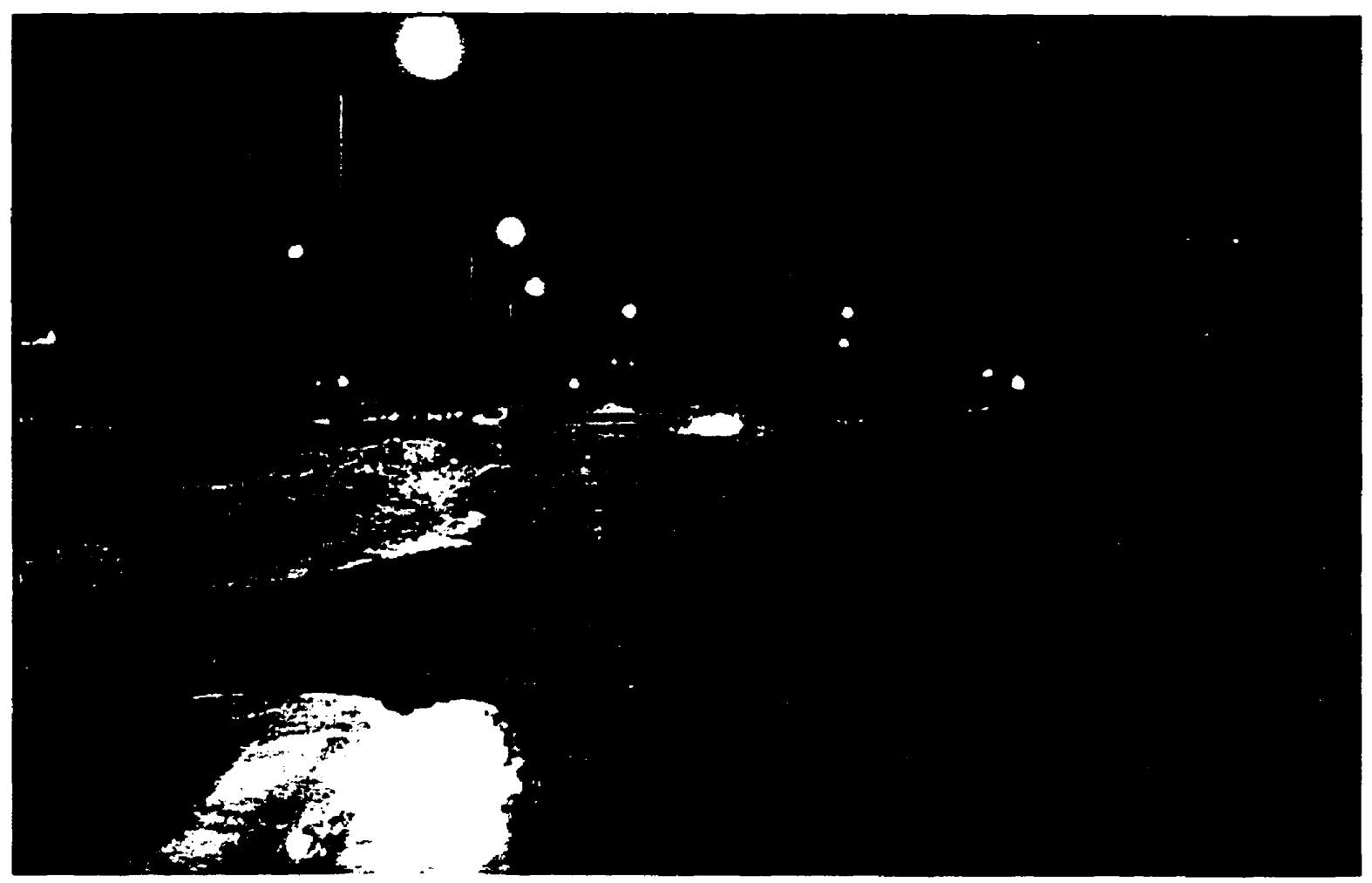

Figure 109 - Demonstration of the Visibility of Targets in the Roadway

In Figure 110, the automobile headlights are turned on. The targets beyond the vehicle are now invisible, and some of the targets in front of the vehicle appear to have reversed 
constrast.

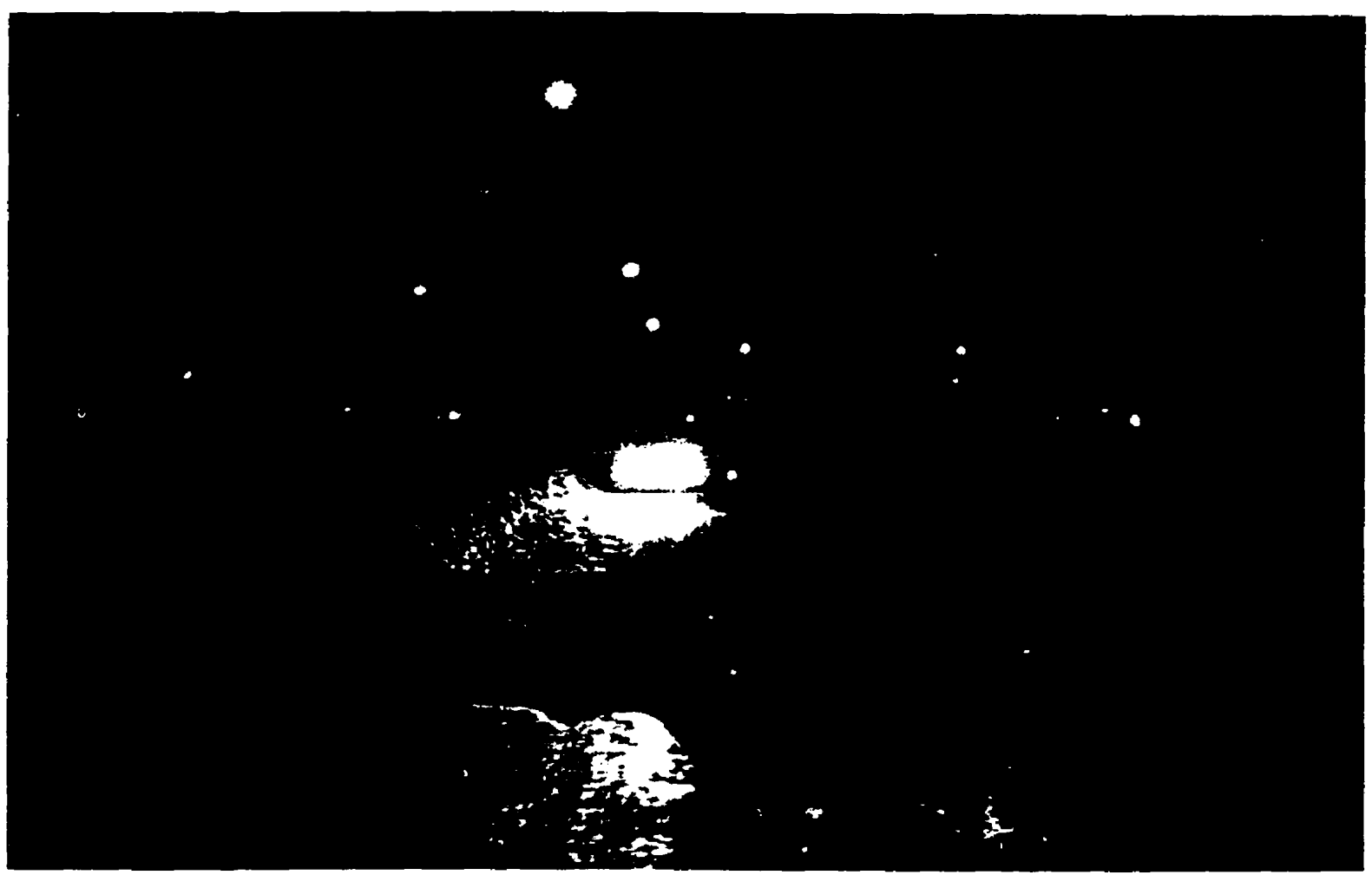

Figure 110 - Visibility Level Demonstration with Glare

In Figure 111, the high beam automobile headlights are turned on. All of the targets but one are now totally invisble in the field of view. Generally, the Visibility Level is calculated without the effect of approaching vehicles. For this demonstration purpose. the added glare shows the impact of the contrast reversal of the target and the visibility of the targets. 


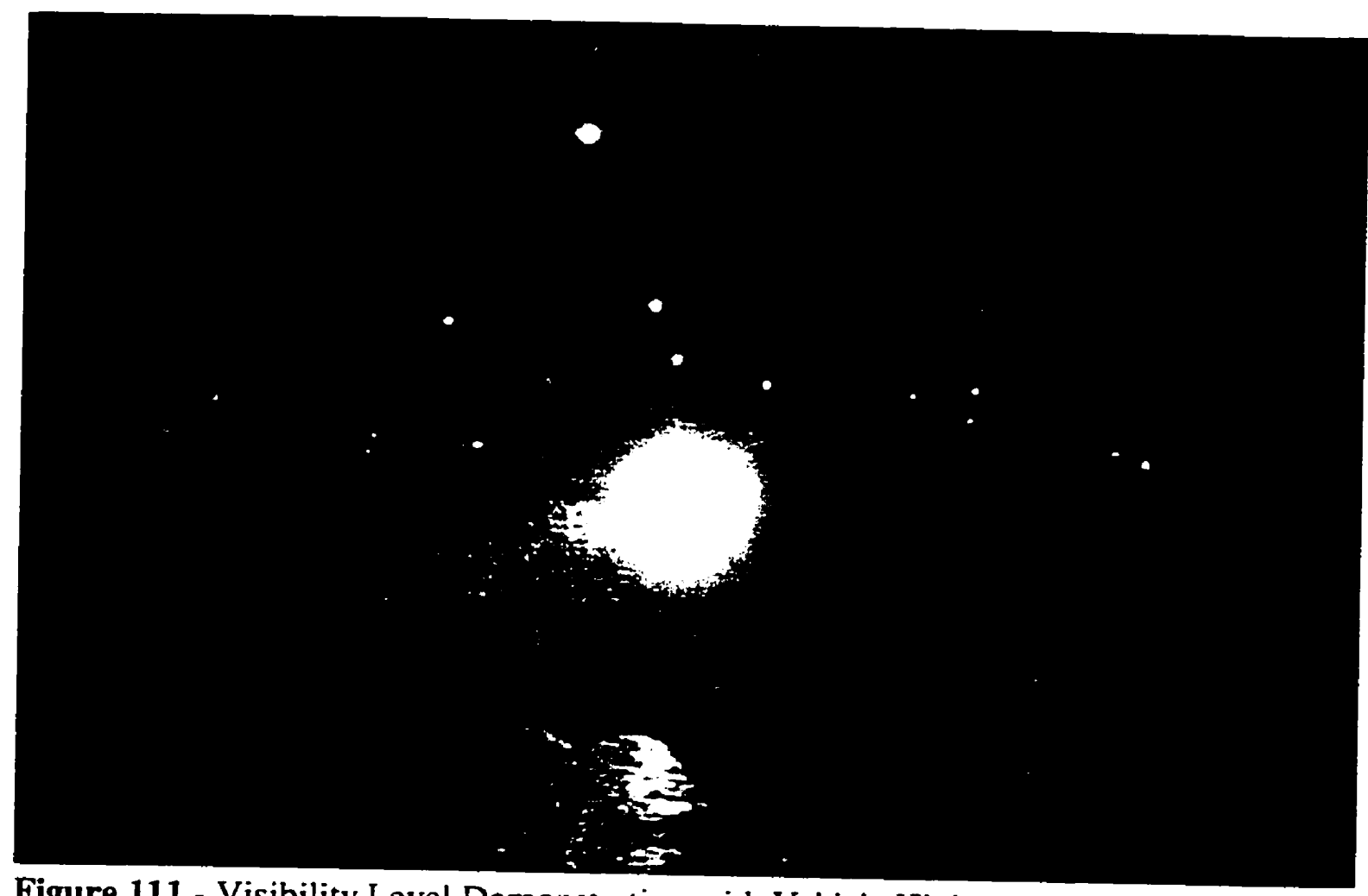

Figure 111 - Visibility Level Demonstration with Vehicle High Beams 


\section{Appendix B}

The contrast threshold, $\mathrm{C}_{\mathrm{th}}$, is calculated by the Adrian[1989] model as follows:

$$
C_{t h}=\frac{\Delta L_{t h}}{L_{B}}
$$

where $\Delta \mathrm{L}_{\mathrm{Ch}}$ is the threshold luminance difference of the object of concern and $\mathrm{L}_{B}$ is the luminance of the background of the object. $L_{B}$ is a measured value where $\Delta \mathrm{I}_{\mathrm{th}}$ is a calculated value. $\Delta \mathrm{L}_{\mathrm{hb}}$ is based on the combination of two laws of visual detection. These laws, Ricco's law and Weber's law, are combined as follows:

$$
\Delta L_{t h}=k \cdot\left(\frac{\sqrt{\Phi}}{\alpha}+\sqrt{L}\right)^{2}
$$

where $\alpha$ is the angular size of the object in minutes of arc and $\mathrm{k}$ is a scaling constant. $\mathrm{k}$ is used to define the probability of detection. For $50 \%$ probability of detection $\mathrm{k}=1$ and for 99.9\% probability of detection $\mathrm{k}=2.6$.

The parameters in this equation are determined according to the following equations: 


$$
\begin{aligned}
& L_{B} \geq 0.6 \mathrm{~cd} / \mathrm{m}^{2} \\
& \sqrt{\Phi}=\log \left(4.1925 \cdot L_{b}^{0.1556}\right)+0.1684 \cdot L_{b}^{0.5867} \\
& \sqrt{L}=0.05946 \cdot L_{b}^{0.466} \\
& L_{b} \leq 0.00418 \mathrm{~cd} / \mathrm{m}^{2} \\
& \log \sqrt{\Phi}=0.028+0.173 \cdot \log \left(L_{b}\right) \\
& \log \sqrt{L}=-0.891+0.5275 \cdot \log \left(L_{b}\right)+0.0227\left(\log \cdot L_{b}\right)^{2} \\
& 0.00418 \mathrm{~cd} / \mathrm{m}^{2}<L_{b}<0.6 c d / \mathrm{m}^{2} \\
& \log \sqrt{\Phi}=-0.072+0.3372 \cdot \log \left(L_{b}\right)+0.0866\left(\log \cdot L_{b}\right)^{2} \\
& \log \sqrt{L}=1.256+0.319 \cdot \log \left(L_{b}\right)
\end{aligned}
$$

This model is valid for a two minute observation time, positive contrast and for a 23 year old observer only.

To adjust for observation time the following factor is used:

Observation Time Factor $=$ OTF $=\frac{a\left(\alpha, L_{b}\right)+t}{t}$ where

$a\left(\alpha, L_{b}\right)=\frac{\sqrt{a(\alpha)^{2}+a\left(L_{b}\right)^{2}}}{2.1}$

where

$$
\begin{aligned}
& a(\alpha)=0.36-0.0972 \cdot \frac{(\log \alpha+0.523)^{2}}{(\log \alpha+0.523)^{2}-2.513 \cdot(\log \alpha+0.523)+2.7895} \\
& a\left(L_{b}\right)=0.36-0.0972 \cdot \frac{\left(\log L_{b}+6\right)^{2}}{\left(\log L_{b}+6\right)^{2}-10.4 \cdot\left(\log L_{b}+6\right)+52.28}
\end{aligned}
$$


Similarly, to adjust for negative contrast the following factor is determined:

$$
\begin{aligned}
& \text { For Positive Contrast } \\
& \text { ContrastPolarityFactor }=C P F=1 \\
& \text { For Negative Contrast } \\
& \text { ContrastPolarityFactor }=C P F=1-\frac{m \cdot \alpha^{-\beta}}{2.4 \cdot \Delta L_{t h(\text { Positive Contrast })}} \\
& \beta=0.6 \cdot L_{b}^{-0.1488} \\
& \text { for } L_{B} \geq .1 \mathrm{~cd} / \mathrm{m}^{2} \\
& m=10^{-10^{-0.125\left(L_{x} L_{b} \cdot 1\right)^{2}-0.0245}} \\
& \text { for } L_{B} \geq .004 \mathrm{~cd} / \mathrm{m}^{2} \\
& m=10^{-10^{-0.05 S\left(L_{x} L_{b} \cdot 1\right)^{2}-0.0245}}
\end{aligned}
$$

Finally, the age of observer is adjusted using the following equation:

$$
\begin{aligned}
& \text { for } 23<\text { Age }<64 \\
& \text { Age Factor }=A F=\frac{(A g e-19)^{2}}{2160}+0.99 \\
& \text { for Age }>64 \\
& A F=\frac{(\text { Age }-56.6)^{2}}{116.3}+1.43
\end{aligned}
$$

The final model of the Threshold Luminance Difference is then: 
$\Delta L_{t h}=A F \cdot C P F \cdot O T F \cdot \Delta L_{t h(R i c c o a n d \text { Weber's Law) }}$ 


\section{Appendix C}

The gonioreflectometer is shown in the photograph in Figure 112.

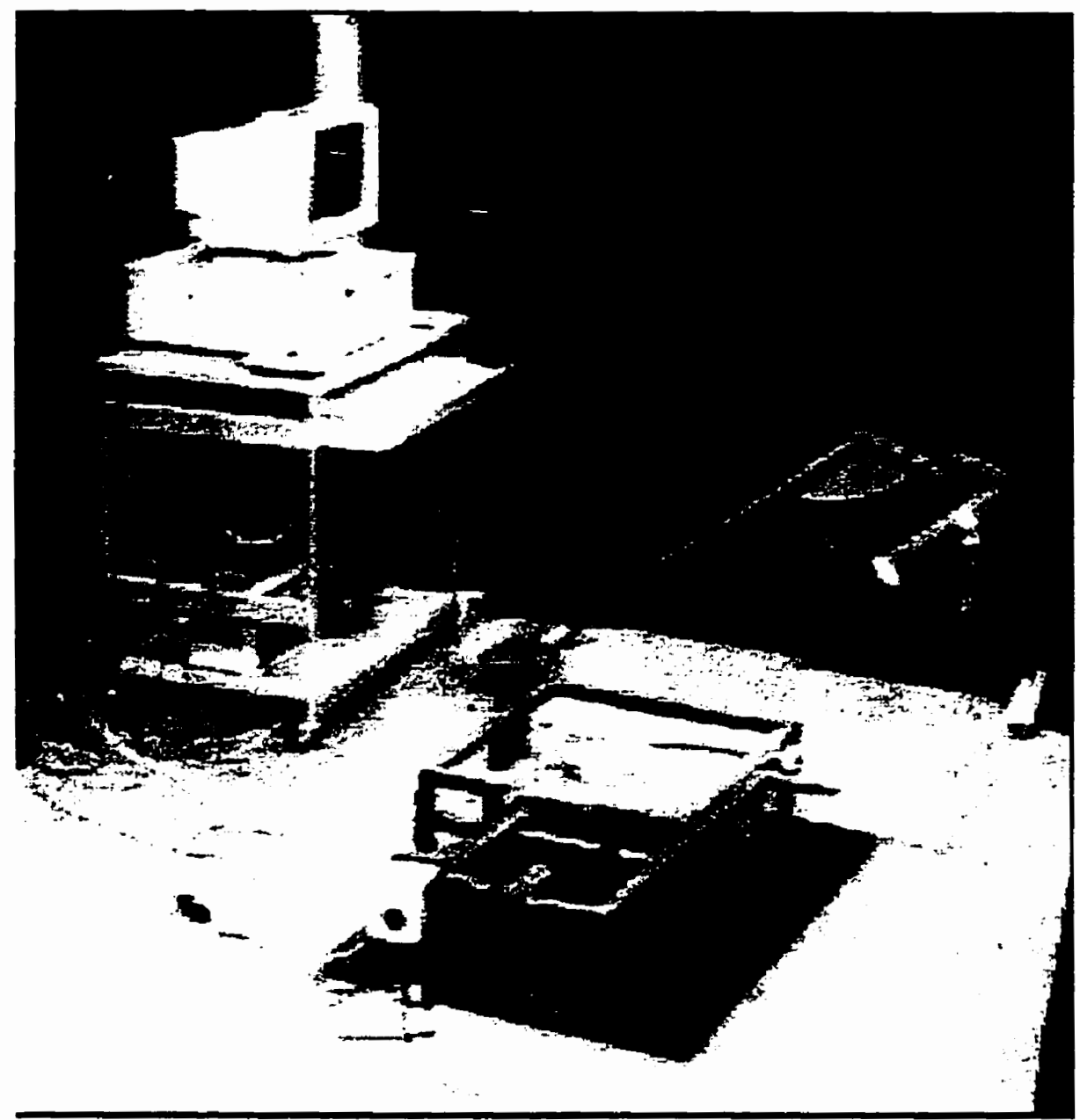

Figure 112 - Photograph of Gonioreflectometer 
